\title{
Epidemiological burden of depression and its impact on adherence to oral hypoglycemic agents and related economic outcomes in patients with type 2 diabetes
}

Iftekhar D. Kalsekar

West Virginia University

Follow this and additional works at: https://researchrepository.wvu.edu/etd

\author{
Recommended Citation \\ Kalsekar, Iftekhar D., "Epidemiological burden of depression and its impact on adherence to oral \\ hypoglycemic agents and related economic outcomes in patients with type 2 diabetes" (2004). Graduate \\ Theses, Dissertations, and Problem Reports. 2115. \\ https://researchrepository.wvu.edu/etd/2115
}

This Dissertation is protected by copyright and/or related rights. It has been brought to you by the The Research Repository @ WVU with permission from the rights-holder(s). You are free to use this Dissertation in any way that is permitted by the copyright and related rights legislation that applies to your use. For other uses you must obtain permission from the rights-holder(s) directly, unless additional rights are indicated by a Creative Commons license in the record and/ or on the work itself. This Dissertation has been accepted for inclusion in WVU Graduate Theses, Dissertations, and Problem Reports collection by an authorized administrator of The Research Repository @ WVU.

For more information, please contact researchrepository@mail.wvu.edu. 
Epidemiological Burden of Depression and its Impact on Adherence to Oral Hypoglycemic Agents and Related Economic Outcomes in Patients with Type 2

Diabetes

Iftekhar D. Kalsekar

Dissertation submitted to the

School of Pharmacy

at West Virginia University

in partial fulfillment of the requirements for the degree of

Doctor of Philosophy

in

Pharmaceutical Systems and Policy

Suresh Madhavan, Ph.D. Chair

Mayur Amonkar, Ph.D.

Eugene Makela, PharmD.

Stratford Douglas, Ph.D.

Virginia Scott, Ph.D.

Department of Pharmaceutical Systems and Policy

Morgantown, West Virginia

2004

Keywords: Medicaid, depression, type 2 diabetes, adherence, and costs

Copyright 2004 Iftekhar D.Kalsekar 


\title{
ABSTRACT \\ Epidemiological Burden of Depression and its Impact on Adherence to Oral Hypoglycemic Agents and Related Economic Outcomes in Patients with Type 2 Diabetes
}

\author{
Iftekhar D. Kalsekar
}

Presence of depression in patients with type 2 diabetes may affect their adherence to oral hypoglycemics and consequently glycemic control and economic outcomes. These potential effects may be more significant when one considers that depression is highly prevalent in patients with diabetes. This study involved two phases. Phase one of the study examined the epidemiological relationship between depression and type 2 diabetes. Phase two of the study examined the impact of depression on patterns of use and adherence to oral hypoglycemics in newly diagnosed type 2 diabetes patients. Impact of depression on expenditures related to type 2 diabetes and overall health care was also estimated. The study also tested the mediating influence of adherence between depression and type 2 diabetes expenditures. Results of phase one of the study indicated that depression was more prevalent in patients with type 2 diabetes as compared to a control group. Results also indicated that females with depression were more likely to develop type 2 diabetes as compared to those without depression. Phase two results indicated that patients without depression had a more favorable pattern of oral hypoglycemic use with a significantly lower proportion of non-depressed patients switching, augmenting, or discontinuing their oral hypoglycemics as compared to depressed patients. Depressed patients were also found to have significantly lower adherence to oral hypoglycemics as compared to non-depressed patients. Multivariate analyses indicated that patients with depression incurred $21.30 \%$ higher type 2 diabetes related costs as compared to non-depressed patients. This difference was primarily due to a higher probability of an ER/hospitalization episode in depressed patients. Similarly, patients with depression had 32.10\% higher overall health care costs as compared to patients without depression. Depression was associated with increased costs in all areas of health care such as ER/hospitalization, outpatient, and prescription costs. Mediation analysis indicated that adherence to oral hypoglycemics was not a mediator between depression and type 2 diabetes related expenditures. Thus, depression could have potentially impacted type 2 diabetes related outcomes directly through a physiological effect on glycemic levels or indirectly through its impact on adherence to other behaviors such as diet or exercise. 


\section{ACKNOWLEDGEMENT}

First and foremost, I would like to thank my major advisor, Dr Suresh Madhavan for his support and guidance throughout my dissertation and graduate studies. A special thanks to him for inspiring me to believe that, " Life is full of golden opportunities carefully disguised as irresolvable problems”. I also extend my thanks to all other committee members; Dr Stratford Douglas, Dr Eugene Makela, Dr Virginia Scott, and Dr Mayur Amonkar for their invaluable input and support on a variety of aspects of my dissertation research. I want them to know that I sincerely appreciated their "excellent" questions during my dissertation defense $\odot$

I also wish to thank Elizabeth Lawton, Commissioner and Peggy King, Pharmacy Coordinator, West Virginia Bureau of Medical Services for their support and approval to conduct this project. I would also like to thank Mary Swim for her programming efforts and Betsy Elswick for her clinical expertise. My sincere thanks to the entire faculty and staff at the School of Pharmacy for their support and encouragement. My fellow graduate students in the department have been both my friends and family in Morgantown. They will always have a special place in my heart. I also appreciate the funding support and mentorship provided by Dr Kim Horn and her Office of Drug Abuse and Intervention Studies (ODAIS). I have made some very special friends and added a whole lot of pounds due to the fun working environment at ODAIS.

Finally, I owe it all to my family. Mummy, Daddy, Muktar bhai, Fajju, and Anu. They have always been there for me through times of hardship and laughter. I am what I am because of all their love and support. I dedicate this dissertation and all that I will ever achieve in life to these special loved ones. 


\section{TABLE OF CONTENTS}

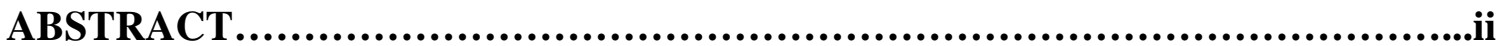

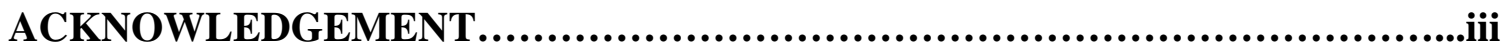

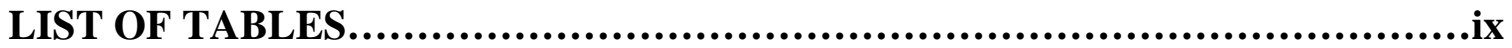

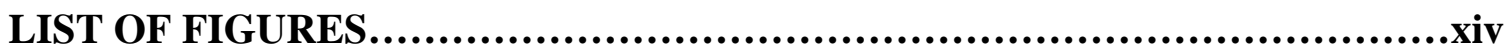

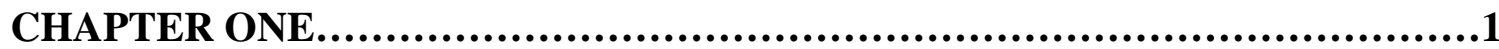

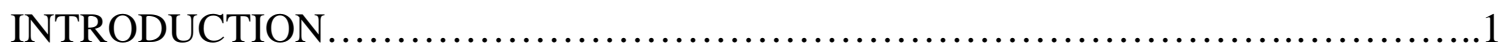

Adherence to Oral Hypoglycemics............................................

Impact of Depression on Adherence..........................................4

Epidemiology of Depression and Diabetes...................................6

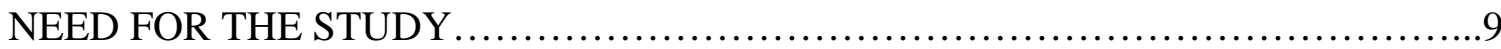

CONCEPTUAL FRAMEWORK.............................................. 12

Selection of Database......................................................

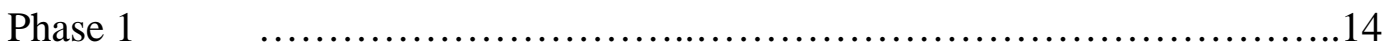

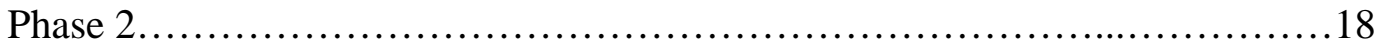

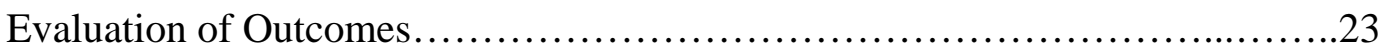

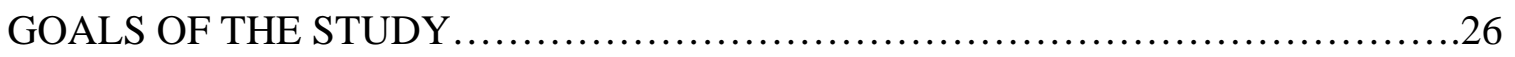

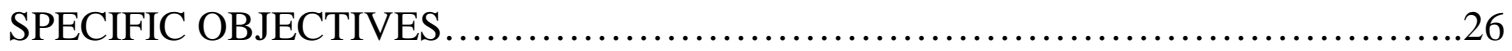

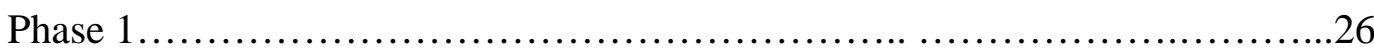

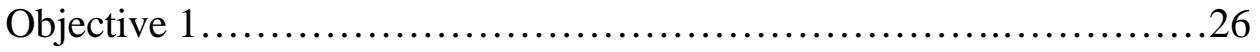

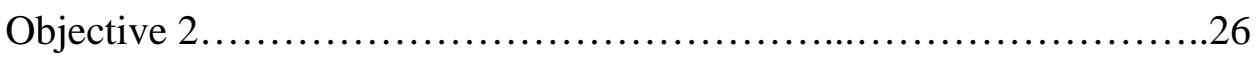

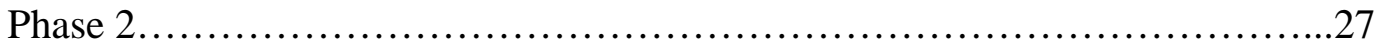




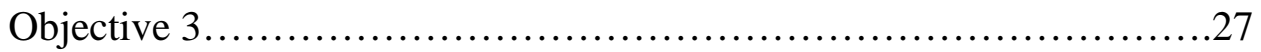

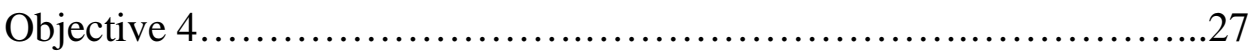

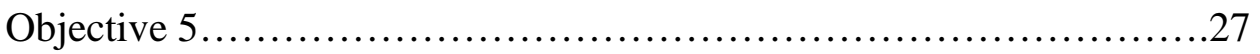

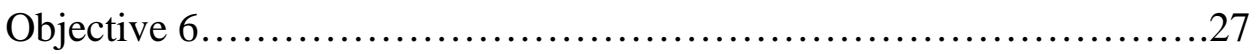

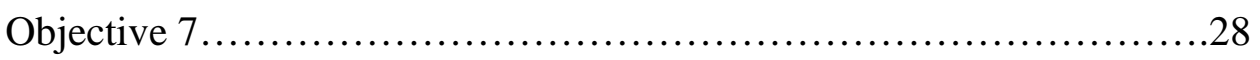

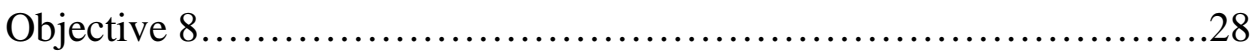

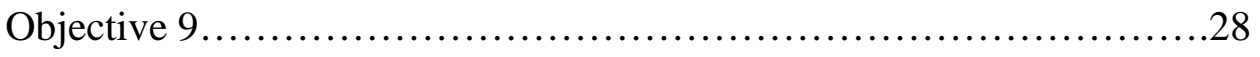

SIGNIFICANCE OF THE STUDY .........................................29

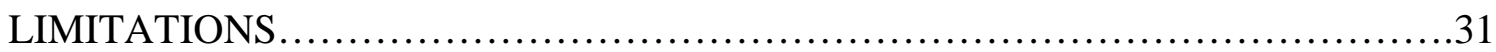

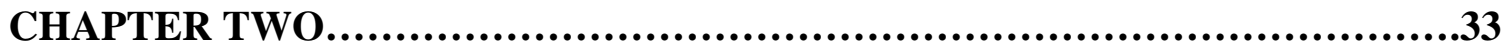

LITERATURE REVIEW..................................................33

Overview of Type 2 Diabetes.........................................33

Diagnostic Tests for Type 2 Diabetes....................................35

Treatment Options................................................. 35

Non-pharmacological Therapy..................................36

Oral Hypoglycemic Agents.......................................36

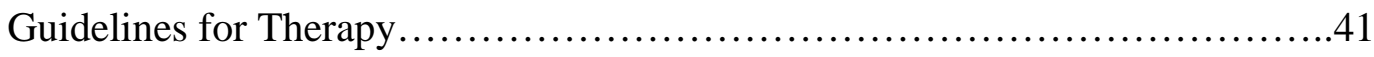

Treatment Goals....................................................42

Impact of Glycemic Control on Type 2 Diabetes Outcomes...................43

Impact of Adherence with Oral Hypoglycemics on Glycemic Control and Type 2

Diabetes Outcomes.................................................45 
Adherence to Oral Hypoglycemics in Patients with Type 2 Diabetes

Factors Affecting Adherence..............................................48

Impact of Depression in Adherence and Outcomes in General...................54

Impact of Depression on Adherence and Outcomes in Type 2 Diabetes...........56

Theories for Impact of Depression on Type 2 Diabetes Outcomes...............61

Epidemiology of Depression and Type 2 Diabetes..........................63

Prevalence of Depression in Patients with Type 2 Diabetes..............63

Incidence of Type 2 Diabetes in Patients with Depression..............67

Overview of the Medicaid Program.......................................70

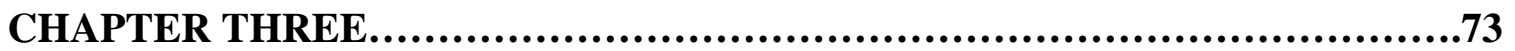

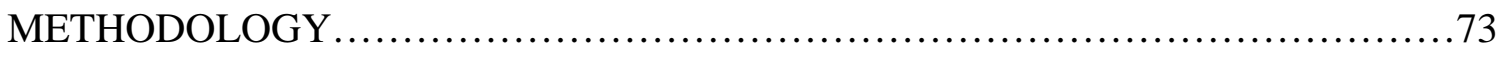

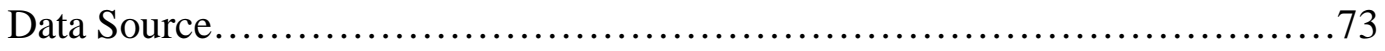

Study Population...................................................... 74

Data Extraction....................................................75

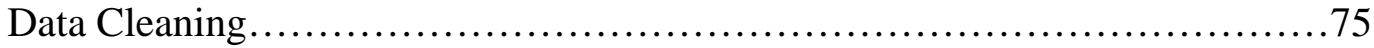

Selection Criteria.......................................................77

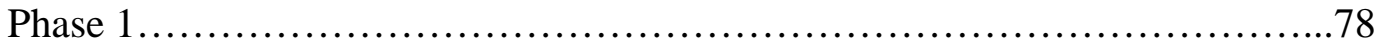

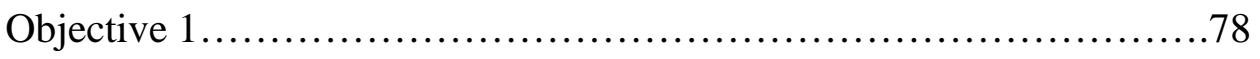

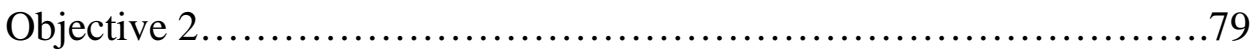

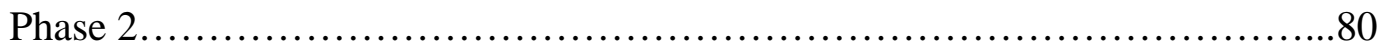

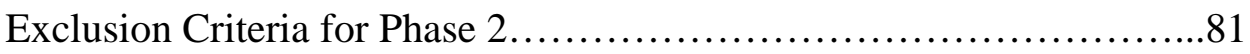

Measurement of Variables........................................82 


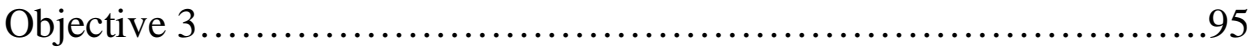

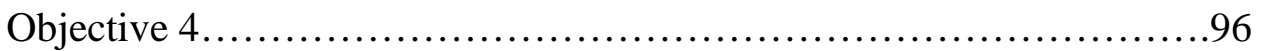

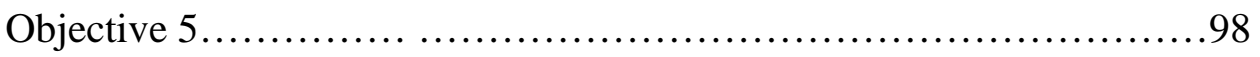

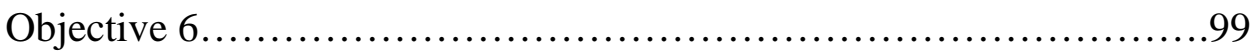

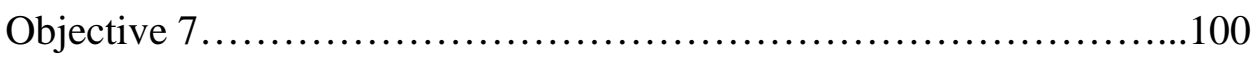

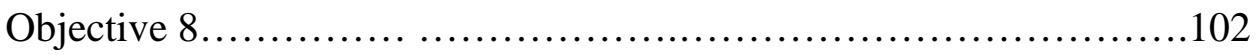

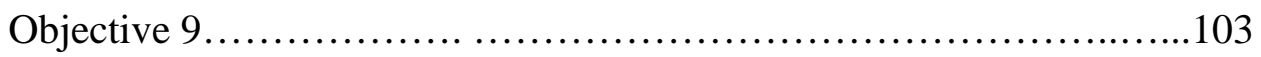

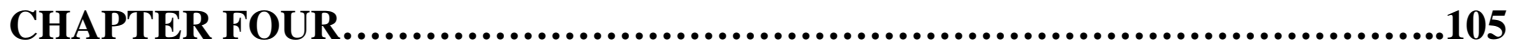

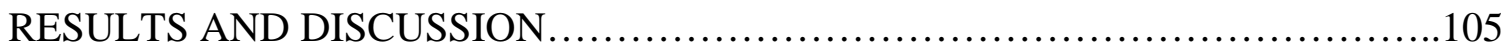

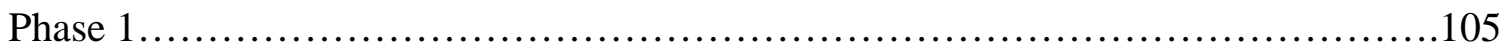

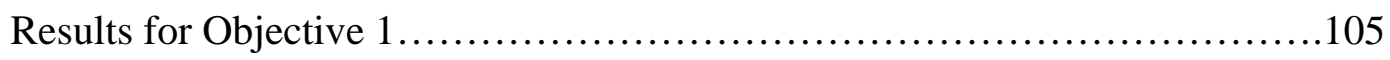

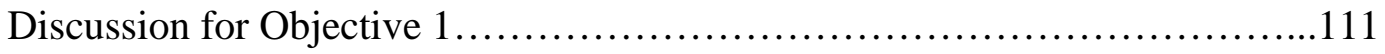

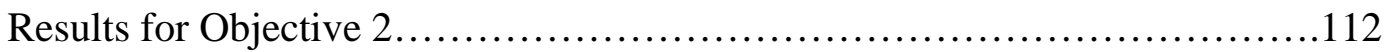

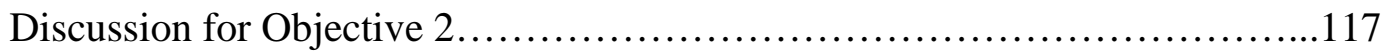

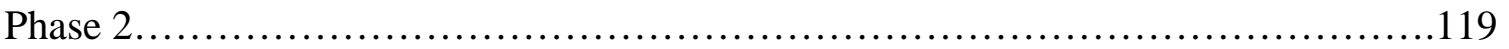

Sample Selection........................................................119

Baseline Characteristics of Newly Diagnosed Type 2 Diabetes Patients........122

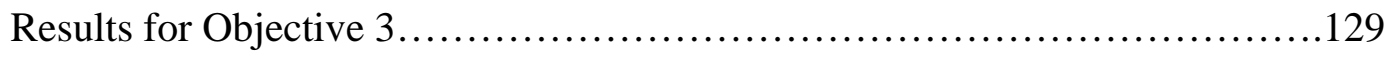

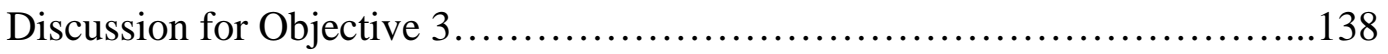

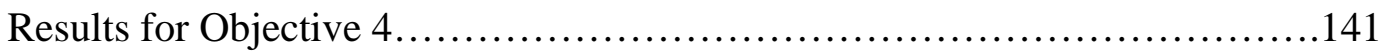

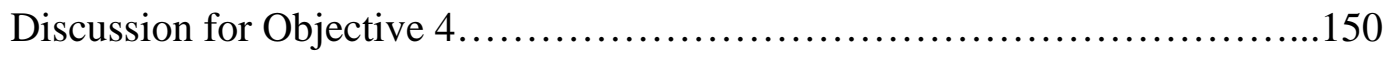

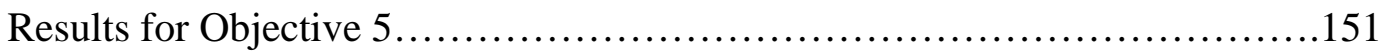




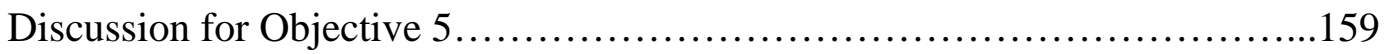

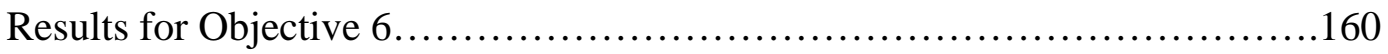

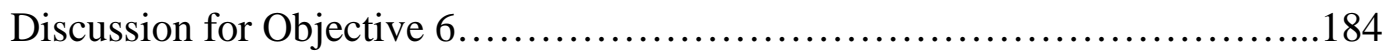

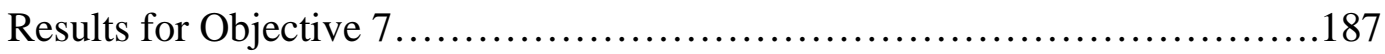

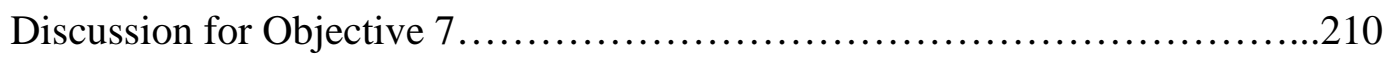

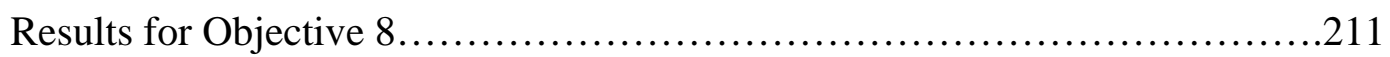

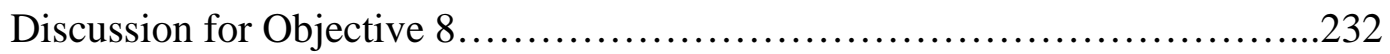

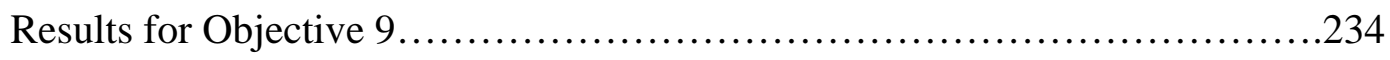

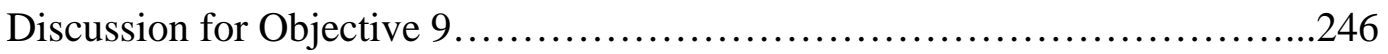

CHAPTER FIVE..................................................................249

SUMMARY AND CONCLUSIONS.........................................249

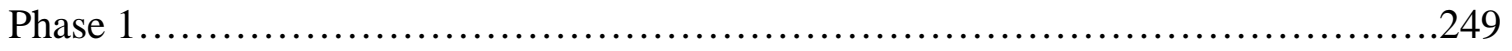

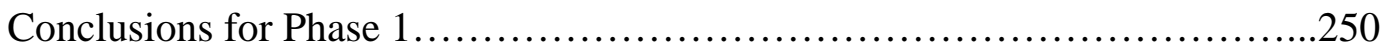

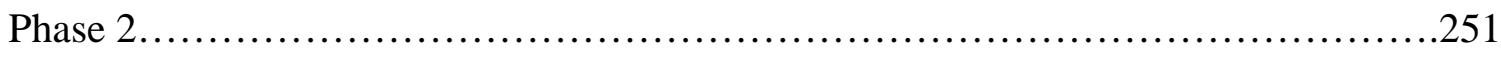

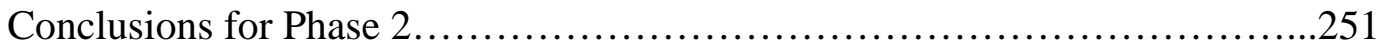

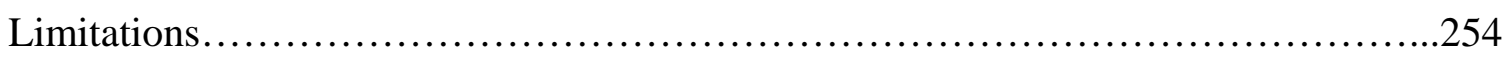

Implications..........................................................257

Directions for Future Research............................................259

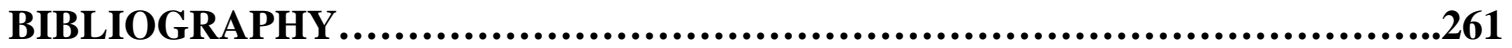

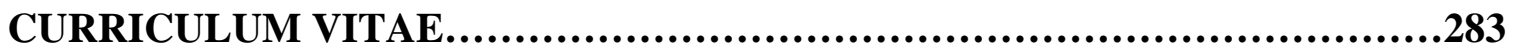




\section{LIST OF TABLES}

Table 1A: Logistic regression model to examine the relationship between type 2 diabetes and presence of co-morbid depression (Model 1) ..................................108 Table 1B: Logistic regression model to examine the relationship between type 2 diabetes and presence of co-morbid depression (Model 2)....................................109 Table 1C: Logistic regression model to examine the relationship between type 2 diabetes and presence of co-morbid depression (Model 3)

Table 2: Logistic regression model to examine the relationship between pre-existing depression and incidence of type 2 diabetes........................................115 Table 3: Logistic regression model to examine the relationship between pre-existing depression and incidence of type 2 diabetes (excluding cases of incident type 2 diabetes

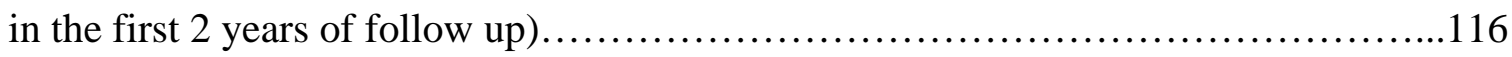

Table 4: Baseline characteristics of newly diagnosed type 2 diabetes patients...........126

Table 5: Pattern of use of oral hypoglycemics in the 12-month follow up period........131 Table 6A: Multinomial Logistic regression for the impact of depression on pattern of oral hypoglycemic use (Switching vs. Non-modification)..............................132 Table 6B: Multinomial Logistic regression for the impact of depression on pattern of oral hypoglycemic use (Augmentation vs. Non-modification)............................134 Table 6C: Multinomial Logistic regression for the impact of depression on pattern of oral hypoglycemic use (Discontinuation vs. Non-modification)........................136

Table 7: Modification of oral hypoglycemics in the 12-month follow up period.........144 Table 8: Logistic regression for the impact of depression on modification of oral

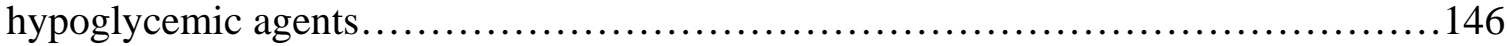


Table 9: Extended Cox proportional hazards model for the impact of depression on time to modification of oral hypoglycemic agent..................................... 148

Table 10: Initiation of insulin therapy in the 12-month follow up period................153

Table 11: Logistic regression for the impact of depression on initiation of insulin

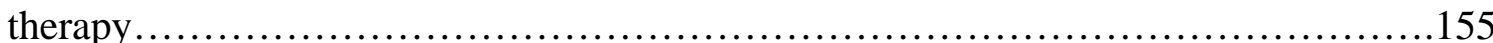

Table 12: Cox proportional hazards model for the impact of depression on time to

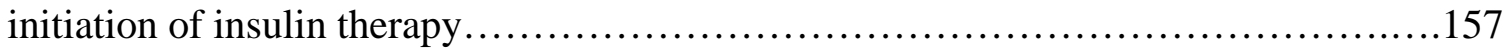

Table 13: Adherence indices in the 12-month follow up period.......................163

Table 14: Ordinary Least Squares (OLS) regression model for the impact of depression on adherence (Reg MPR-1) to oral hypoglycemics...............................164

Table 15: Ordinary Least Squares (OLS) regression model for the impact of depression on adherence (Reg MPR-2) to oral hypoglycemics.............................166

Table 16A: Multinomial Logistic regression for the impact of depression on adherence (Reg MPR-1) to oral hypoglycemics.........................................168

Table 16B: Multinomial Logistic regression for the impact of depression on adherence (Reg MPR-1) to oral hypoglycemics..........................................170 Table 16C: Multinomial Logistic regression for the impact of depression on adherence (Reg MPR-1) to oral hypoglycemics..........................................172 Table 16D: Multinomial Logistic regression for the impact of depression on adherence (Reg MPR-1) to oral hypoglycemics......................................174 Table 17A: Multinomial Logistic regression for the impact of depression on adherence (Reg MPR-2) to oral hypoglycemics.......................................176 
Table 17B: Multinomial Logistic regression for the impact of depression on adherence (Reg MPR-2) to oral hypoglycemics 178

Table 17C: Multinomial Logistic regression for the impact of depression on adherence (Reg MPR-2) to oral hypoglycemics.... .180

Table 17D: Multinomial Logistic regression for the impact of depression on adherence (Reg MPR-2) to oral hypoglycemics. .182

Table 18: Utilization and expenditures for type 2 diabetes in the 12-month follow up period.

Table 19: Semi-log Ordinary Least Squares (OLS) regression model for the impact of depression on total diabetes costs

Table 20A: Logistic regression for the impact of depression on having diabetes related ER/Hospitalization episode in the 12-month follow-up period.

Table 20B: Semi-log Ordinary Least Squares (OLS) regression model for the impact of depression on diabetes related ER/hospitalization costs. 196 Table 21A: Logistic regression for the impact of depression on having a diabetes related outpatient episode. 198 Table 21B: Semi-log Ordinary Least Squares (OLS) regression model for the impact of depression on diabetes related outpatient costs..................................200 Table 22: Semi-log Ordinary Least Squares (OLS) regression model for the impact of depression on diabetes prescription costs...................................202 Table 23: Negative binomial model for the impact of depression on number of diabetes related ER/Hosp episodes. 204 
Table 24: Negative binomial model for the impact of depression on number of diabetes related physician office visits .206

Table 25: Negative binomial model for the impact of depression on number of diabetes prescriptions. 208

Table 26: Utilization and expenditures for overall health care (excluding those due to depression) in the 12-month follow up period. .215 Table 27: Semi-log Ordinary Least Squares (OLS) regression model for the impact of depression on total healthcare costs (excluding depression costs) ..................216 Table 28A: Logistic regression for the impact of depression on having an ER/Hospitalization episode (excluding depression).... 218 Table 28B: Semi-log Ordinary Least Squares (OLS) regression model for the impact of depression on total ER/hospitalization costs (excluding depression).... .220 Table 29: Semi-log Ordinary Least Squares (OLS) regression model for the impact of depression on total outpatient costs (excluding depression).

Table 30: Semi-log Ordinary Least Squares (OLS) regression model for the impact of depression on total prescription costs (excluding depression) $\ldots \ldots \ldots \ldots \ldots \ldots \ldots \ldots \ldots . . .224$ Table 31: Negative binomial model for the impact of depression on number of overall ER/hospitalization episodes (excluding depression)............................226 Table 32: Negative binomial model for the impact of depression on number of overall

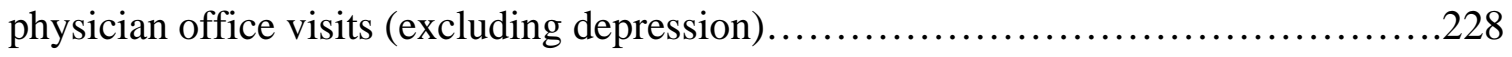
Table 33: Negative binomial model for the impact of depression on number of overall

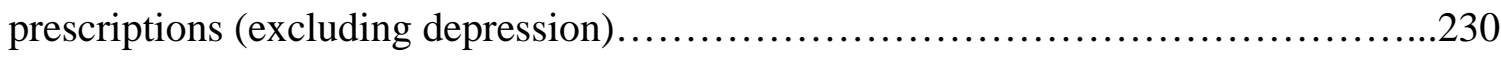


Table 34: Semi-log Ordinary Least Squares (OLS) regression model for the impact of adherence (MPR-1) on total diabetes related costs.................................238 Table 35: Logistic regression for the impact of adherence (Reg MPR-1) on having a type 2 diabetes related ER/hospitalization episode.....................................240 Table 36: Semi-log Ordinary Least Squares (OLS) regression model for the impact of adherence (Reg MPR-2) on total diabetes related costs............................242 Table 37: Logistic regression for the impact of adherence (Reg MPR-2) on having a type 2 diabetes related ER/hospitalization episode. 


\section{LIST OF FIGURES}

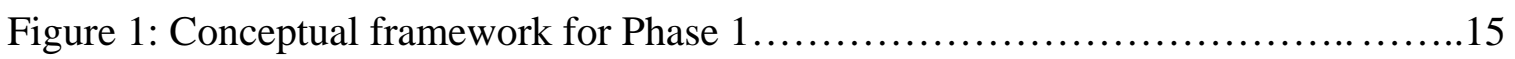

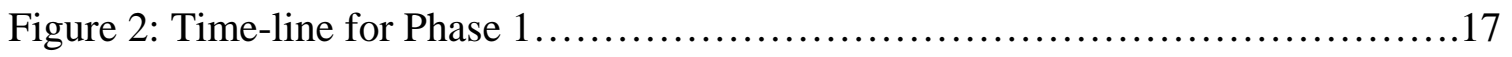

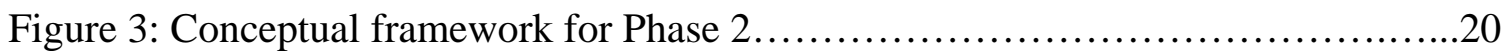

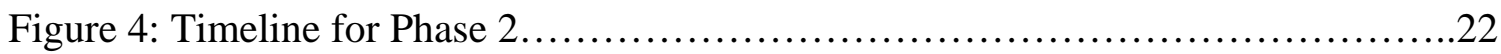

Figure 5: Algorithm for sample attrition of patients with newly diagnosed type 2 diabetes

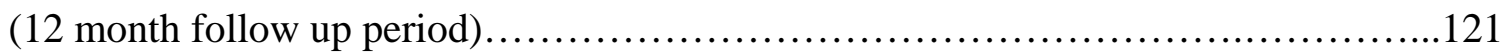

Figure 6: Kaplan-Meier Survival Curves for time to modification of oral hypoglycemic

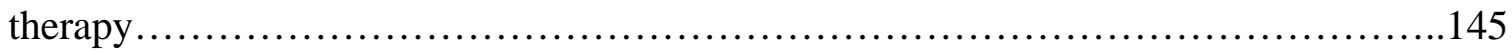

Figure 7: Kaplan-Meier Survival Curves for time to initiation of insulin therapy........154 


\section{CHAPTER ONE}

\section{INTRODUCTION}

An estimated 17 million Americans are affected by diabetes. The American Diabetes Association (ADA) has estimated the total cost attributable to diabetes to be $\$ 132$ billion in 2002, which included \$ 91.8 billion in direct medical and treatment costs. ${ }^{1}$ The prevalence rates of diabetes in Medicaid programs have been reported to be nearly twice that of the US population as a whole. Prevalence based costs of illness studies have shown that the per capita mean cost of patients with diabetes in Medicaid were nearly $\$ 8,000$ per year; approximately four times the annual cost of a Medicaid enrollee without diabetes. $^{2}$

Diabetes mellitus can be divided into type 2 diabetes (formerly called non-insulin dependent diabetes mellitus) and type 1 diabetes (formerly known as insulin dependent diabetes mellitus). Type 2 diabetes is more prevalent and accounts for more than $90 \%$ of diabetes cases. ${ }^{3}$ Data from the National Medical Expenditure Panel Survey (MEPS) showed that inpatient hospital care for diabetes accounted for $63 \%$ of the total health care expenditure for diabetics. ${ }^{4}$ Although the cost breakdown estimates for Medicaid are not available, one can assume that even in Medicaid programs, diabetic complications account for a major fraction of the total costs attributable to type 2 diabetes.

Over the past decade large-scale studies have demonstrated that tight glycemic control greatly reduces the frequency and severity of long-term diabetes related complications. In the 10-year United Kingdom Prospective Diabetes Study (UKPDS), intensive glycemic control resulted in A1C levels that were significantly lower than in type 2 diabetes patients on conventional therapy. ${ }^{5}$ The UKPDS data showed a continuous 
relationship between the risks of microvascular complications and glycemia, such that for every percentage point decrease in $\mathrm{A} 1 \mathrm{C}$ there was a $35 \%$ reduction in the risk of complications.

The importance of tight glycemic control has been also recognized by key US organizations. The National Committee for Quality Assurance (NCQA) and the Foundation for Accountability (FACCT) encourage health care delivery systems to provide appropriate diabetes care by maintaining tight glycemic control in their enrollees. The American Diabetes Association (ADA) recommends measuring A1C every three months and maintaining this value below $7 \%$ to prevent future complications. ${ }^{6}$ However, in one study, only $20 \%$ of health maintenance organization members with diabetes met these ADA standards. ${ }^{7}$

Studies using measures of adherence such as pill count and refill information have demonstrated associations between drug adherence and glycemic control. Diehl and associates $^{8}$ used pill counts to assess adherence to oral hypoglycemics in patients with type 2 diabetes and found a trend towards higher fasting blood glucose in patients taking less than $80 \%$ of the prescribed doses. In addition, both Chousa and associates ${ }^{9}$ and Peterson and associates ${ }^{10}$ used pill count and self-reported measures of adherence to demonstrate an association between adherence and A1C levels. In a recent study conducted by Schectman and associates ${ }^{11}$ in an indigent population using pharmacy claims, glycemic control and its improvement over time were strongly associated with adherence to diabetes medication measured on the basis of refill information. Thus, evidence in the literature clearly links improved glycemic control through better adherence to drug therapy in patients with type 2 diabetes. 


\section{Adherence to Oral Hypoglycemics}

There is a large literature demonstrating a strong association between adherence to medication and outcomes. Adverse outcomes resulting from non-adherence including increased use of emergency room and inpatient services account for a large proportion of the drug-related morbidity and mortality estimated to cost $\$ 76.6$ billion in the US annually. ${ }^{12}$ Non-adherence to oral hypoglycemic agents can also result in dire consequences for patients with type 2 diabetes. A review of 13,309 patients with type 2 diabetes from the UK Mediphys database documented adherence and clinical events between 1991 and 1997. ${ }^{13}$ Patients who discontinued their oral hypoglycemic agents were approximately twice as likely to experience an emergency medical event and had a mortality rate that was three times that of continuers. Thus, adherence to oral hypoglycemic agents assumes great importance in preventing future complications and controlling health care expenditures in patients with type 2 diabetes.

However, it is increasingly recognized that patients are non-adherent to many aspects of diabetes self-management including sufficient intake of appropriate oral antidiabetics. Approximately $10-30 \%$ of patients affected by type 2 diabetes withdraw from their prescribed regimen within one year of diagnosis. Of the remaining patients, nearly a quarter take insufficient medication thus failing to achieve appropriate glycemic levels. ${ }^{14}$ An observational study of subjects enrolled in an HMO suggested that $31 \%$ of type 2 diabetes patients on oral hypoglycemic monotherapy did not refill any drug in the following year. ${ }^{15}$ A similar study of a US pharmacy claims database found low 12-month persistence ranging from $31 \%$ for alpha-glucosidase inhibitors to $60 \%$ for metformin. ${ }^{16}$ Similar results were found in a study conducted in Scotland which showed that adherence 
to oral hypoglycemic agents was sub-optimal, with half of patients treated with metformin and over one third of those treated with sulphonylureas obtaining insufficient drug supplies for adequate drug coverage. ${ }^{17}$

Medication non-adherence is a major concern in all chronic conditions especially in symptom free patients. Studies conducted in other Medicaid populations have found that newly diagnosed type 2 diabetes patients were less adherent than patients consuming oral hypoglycemics for a longer period of time. ${ }^{18}$ This may be due to the lack of diabetic complications and other symptoms in the initial stages of type 2 diabetes. Newly diagnosed patients do not feel as vulnerable to the development of complications and hence are less likely to comply with their medication regimen. Some patients may also be in a stage of denial and fail to acknowledge the presence of type 2 diabetes leading to decreased medication adherence. Newer patients may also be less informed and less supervised regarding their condition by physicians and other health care providers due to lack of diabetic complications. Thus, newly diagnosed patients are at a considerably higher risk for non-adherence with diabetes medication, subsequently leading to inadequate glucose control and diabetic complications.

\section{Impact of Depression on Adherence}

There are a plethora of reasons for patients to be non-adherent to their medications. These reasons may range from their disbelief in the efficacy and adverse events associated with treatment to various socio-economic constraints. A number of health behavior models such as the Health Belief Model have tried to examine these factors related to adherence. ${ }^{19}$ Specifically in patients with type 2 diabetes, cost of therapy, severity of diabetes, presence of co-morbid conditions, use of insulin therapy, 
interactions with health care providers, and complexity of drug regimens have been shown to be factors associated with adherence to oral hypoglycemic therapy, diet and exercise. $^{8,20-22}$

In addition to these factors, presence of co-morbid depression can be an important factor leading to lack of adherence in these patients. The precise mechanism by which depression might affect medication adherence is complex. Several features of depression can have detrimental effects on adherence with medications including poor motivation, decrements in attention, memory, and cognition, decreased self-care, and even intentional self-harm. ${ }^{23}$ As depressive symptoms are normally associated with a feeling of hopelessness and pessimism, they may hinder adherence to medical regimen. In addition, depression is often accompanied by social isolation and withdrawal thus possibly making an individual highly susceptible to non-adherent behavior. Also, most importantly depression may impact an individuals cognitive functioning resulting in forgetfulness to adhere to complex treatment regimen. Some studies have also indicated that depression has been associated with greater sensitivity to unpleasant side effects from medications. ${ }^{24}$ Depression also adversely affects satisfaction with care, which itself predicts poor adherence to medical regimens. ${ }^{25,26}$

A few studies have examined the association between depression and treatment adherence. It is well recognized that adherence to antidepressants is very poor; with only $40 \%$ of those receiving an antidepressant prescription completing the nine-month recommended treatment. ${ }^{27}$ There is also a lack of adherence to other medical regimen in patients with depression. A recent meta-analysis of 12 published studies confirmed the same demonstrating that depressed patients were three times as likely as non-depressed 
patients to be non-adherent to physician recommendations. ${ }^{23}$ In the only study ${ }^{28}$ examining the impact of depressive symptoms on adherence in patients with diabetes, the depressive symptoms were assessed using the Hopkins Symptom Checklist-90 Revised; adherence was assessed by self-report and by using an automated data collection system for refills of oral hypoglycemic agents. The results demonstrated that diabetes patients with higher levels of depressive symptoms had more lapses in filling their prescriptions for hypoglycemic agents. Assessing the extent to which non-adherence to a medication regimen for a chronic condition such as type 2 diabetes may be a result of a treatable condition such as depression can be important in improving patient adherence and finally improving outcomes of treatment.

\section{Epidemiology of Depression and Diabetes}

The potential effects of depression on outcomes associated with management of type 2 diabetes may be even more significant when one considers that depression is highly prevalent in patients with diabetes. A recent meta-analysis of 42 studies indicated that the odds of depression in patients with diabetes were twice that of a comparison group of patients without diabetes. ${ }^{29}$ A sub-analysis of seven studies including only patients with type 2 diabetes indicated that patients with type 2 diabetes were nearly two and a half times more likely to have co-morbid depression as compared to patients without type 2 diabetes.

In addition to prevalence of co-morbid depression in patients with type 2 diabetes, it is also important to consider the epidemiological measure of incidence. Incidence has the advantage of establishing the temporal order and disentangling the causes of onset from influences on chronicity. Diabetes causes biochemical changes as well as may 
require psychosocial adjustments that may lead to depression. On the other hand, depression is related to changes in nutrition and exercise that may contribute to risk for diabetes; and there may be somatic aspects of depression such as a change in the immune or vascular system that contributes to enhanced risk for diabetes onset. ${ }^{30,31}$ Neurohormonal changes induced by depression such as increase in levels of cortisol and catecholamines can lead to insulin resistance and to the development of type 2 diabetes. Depressed individuals are less likely to be physically active and eat a healthy diet. These adverse health behaviors may in turn lead to obesity and subsequently to the development of diabetes. Evidence from a few prospective studies indicates that depression doubles the risk of incident type 2 diabetes even after controlling for other risk factors. Controlling for multiple risk factors, Eaton and colleagues ${ }^{32}$ have shown that community respondents with major depression, diagnosed using the National Institute of Mental Health's Diagnostic Interview Schedule, had an estimated relative risk of 2.23 of developing type 2 diabetes over a 13 -year period. Kawakami and associates ${ }^{33}$ also demonstrated in a sample of 2,764 male employees of an electrical company in Japan that significant depressive symptoms, measured using the Zung Self-Rating Depression scale, were associated with a 2.3 times increased risk of developing type 2 diabetes compared to non depressed employees after controlling for known risk factors. However, these studies had a small number of incident case ( $n=89$ and $n=43$, respectively) and thus had limited statistical power. A couple of other studies used data from the National Health and Nutrition Examination Epidemiologic Follow-up Study (NHEFS) to test the relationship between depression and incidence of diabetes. ${ }^{34,35}$ These studies used the NHEFS cohorts and measured depression using self-reported scales such as the Center 
for Epidemiologic Studies Depression Scale (CES-D) and the General Well-Being Depression subscale. These studies had variable results with only one of the studies demonstrating a significant and independent role of depressive symptoms in the development of diabetes.

Thus, the literature indicates the possibility of higher rates of pre-existing depression in patients with type 2 diabetes, which may subsequently lead to lack of adherence with anti-diabetic medications resulting in poorer outcomes. In recent years, a number of new oral antidiabetics have been approved including acarbose, glimepride, metformin, miglitol, repaglinide, and troglitazone. These compounds have been shown to be effective in achieving tight glycemic control as either monotherapy or in combination with other oral antidiabetics. However, inadequate drug adherence due to the presence of pre-existing depression would negate the benefits of these expensive antidiabetic agents. All these findings cause increased concern regarding increased morbidity, mortality and expenditures associated with the management of new patients with type 2 diabetes. 


\section{NEED FOR THE STUDY}

Epidemiological studies in the literature regarding the association of depression and diabetes were conducted in a mix of type 1 and type 2 diabetes patients. ${ }^{29}$ These issues have not been explored exclusively in type 2 diabetes patients. Also, most of these studies relied on self-reported data for identification of diabetes and depression. ${ }^{29}$ Studies in the literature have used a variety of scales to measure the self-report of depressive symptoms and each scale measures depressive symptoms over a different time interval. ${ }^{29}$ These self-reported scales can provide an overestimation of depression as symptoms related to diabetes may be misclassified as those due to depression. Also, these measures are not accurate predictors of psychiatric diagnosis as transient depression symptoms may reflect a situational response to current life events. Some of the selfreported depression scales such as the CES-D scale do not distinguish between general anxiety and depression and are also inaccurate in identifying patients with major mood disorders.

These epidemiological studies also suffered from selection bias, as they have been conducted in specialized settings that treat patients with problematic diabetes. Moreover, potential confounders such as sex, age, race, or concomitant medical illness were not addressed. The published studies also had limited statistical power and generalizability due to low sample sizes. In addition, these prevalence and incidence rates may be presumably higher in an indigent population such as enrollees in Medicaid. Hence, there is a need to obtain estimates of clinically diagnosed depression in patients with type 2 diabetes in a population of lower socio-economic status such as Medicaid enrollees. 
Studies of the relationship between depression and adherence have used a variety of approaches to assess depression or depressive symptomatology (ranging from questionnaires such as the CES-D or the Beck Depression Inventory, to clinical interviews). Also, different aspects of adherence (adherence to diet, exercise, glucose monitoring, and medications), different criteria for defining adherence, and different approaches to measuring adherence have been used. It is also possible that the association between depression and adherence is artificially created due to the methods of measuring adherence. When asked to self-report their levels of adherence, depressed patients may have poor self-perceptions and underestimate their actual level of adherence. Thus, it is important to use objective measures of adherence (such as pill counts or refill information) in assessing the relationship between depression and adherence. Also, a lower socio-economic population may have greater barriers to adherence thwarting efforts at improving care and outcomes. ${ }^{11}$ As patients enrolled in Medicaid generally have a greater burden of chronic disease, less vigorous treatment, and poorer disease outcomes, it will be appropriate to focus efforts on improving adherence in such populations.

Studies in the literature examining the impact of depression on adherence to treatment regimen were not necessarily restricted to drug therapy and were all co-relational in nature. Cross sectional studies are not methodologically capable of establishing directional effects. The patient population in these studies consisted of a mix of both prevalent and incident type 2 diabetes cases. If such cross-sectional data is used to study the relation between adherence and depression, the chicken and egg scenario arises, wherein one is not able to assess whether depression caused a decrease in medication 
adherence or a decrease in adherence with medications aggravated conditions associated with type 2 diabetes leading to depression. This lack of causality and especially the biases in model estimation due to presence of endogeneity makes it imperative to observe the data longitudinally. Also, the adherence behavior of those newly diagnosed with diabetes and starting treatment for the first time may differ markedly from that of survivor cohorts (i.e. those patients who stay under treatment). The problem of nonadherence may be especially common in the years immediately after diagnosis when many patients with newly diagnosed type 2 diabetes may not yet have accepted or adjusted to their diagnosis. ${ }^{18}$ Hence, there is a need for large longitudinal studies in which depression, adherence, and health outcomes are examined and the direct effects of depression and the indirect effects of depression through patient adherence on health outcomes are explored in patients with newly diagnosed type 2 diabetes. 


\section{CONCEPTUAL FRAMEWORK}

The study will be conducted in two distinct phases.

- Phase 1 will examine the epidemiological relationship between depression and type 2 diabetes

- Phase 2 will examine the impact of depression on adherence with oral hypoglycemics and establish causal pathways for the impact of depression on outcomes related to management of type 2 diabetes

\section{Selection of Database}

The study will be conducted using claims data, which provides a number of advantages. Claims databases are typically used for billing purposes and thus have comprehensive information regarding health care utilization and costs. The presence of separate files for outpatient visits, emergency room visits, hospitalizations, and pharmacy records allow the examination of all components of health care and the relationships between them. Availability of eligibility information also provides the opportunity to conduct longitudinal studies following continuously eligible patients for extended periods of time. One of the biggest advantages of claims data is their non-intrusiveness. As the providers and patients are never contacted, the chances of a Hawthorne effect (improvement of performance under observation) in the study are minimized. Also, identification of disease conditions, and utilization parameters are not self-reported and hence more likely to be accurate. Claims data research is less expensive and time consuming as compared to other type of research like clinical trials. It also provides large samples for sufficient power in statistical tests. Thus, claims data provide an opportunity 
for inexpensive, non-intrusive research providing high statistical power in real world settings. ${ }^{36}$

West Virginia Medicaid claims data will be used to meet the objectives of this study. Medicaid enrollees have comprehensive prescription benefits and out-of system use is rare. Thus, Medicaid prescription data is a more valid and comprehensive source than either patient recall or physician's prescribing records. Pharmacy refill data thus provide a valid approach to measuring drug adherence, which is an important variable in the methodological framework of our study. In measuring adherence from refill information, an assumption is made that a filled prescription results in ingestion of a full supply of the drug. However, as in a population enrolled in Medicaid procurement of drugs from other sources is highly unlikely, a prescription which is not refilled can be considered a good indicator of a prescription not taken and thus of non-adherence. There can also be issues related to the validity of the prescription claims information. However, a validation study that compared Medicaid data with its primary sources such as hospitals, physicians, and pharmacies showed that medication data were of high quality. ${ }^{37}$ In contrast, self reported adherence is prone to recall bias and tends to be overestimated because of desires of social desirability on the part of the respondents. Also expensive adherence assessment methods such as Medication Event Monitoring System (MEMS) caps may be subject to the Hawthorne effects. ${ }^{38}$ 


\section{Phase 1}

The conceptual framework regarding the relationship between pre-existing depression and incidence of type 2 diabetes is demonstrated in Figure 1. Previous literature indicates the impact of pre-existing depression on increasing the incidence of type 2 diabetes. ${ }^{39}$ A possible explanation for this relationship is the presence of detection bias as individuals with depressive symptoms may be more likely to seek medical care than individuals without symptoms, and therefore would have a greater opportunity for being diagnosed with diabetes than those without symptoms leading to an increased prevalence and incidence of type 2 diabetes among patients with depression. ${ }^{40,41}$ Another possible explanation is that depression can lead to increased incidence due to physiological reasons such as neuro-hormonal changes leading to insulin resistance and increased glucose levels. ${ }^{42,43}$ Behavioral changes induced by depression such as physical inactivity, smoking, and unhealthy diet are also considered risk factors for type 2 diabetes. $^{44,45}$ These adverse health behaviors may also lead to obesity and subsequently to the development of type 2 diabetes. Being overweight is associated with onset of type 2 diabetes and depressed individuals suffer from problems with appetite and weight gain. However, one can also view obesity as a mediating variable in the relationship between depression and onset of type 2 diabetes. 
Figure 1: Conceptual framework for Phase 1

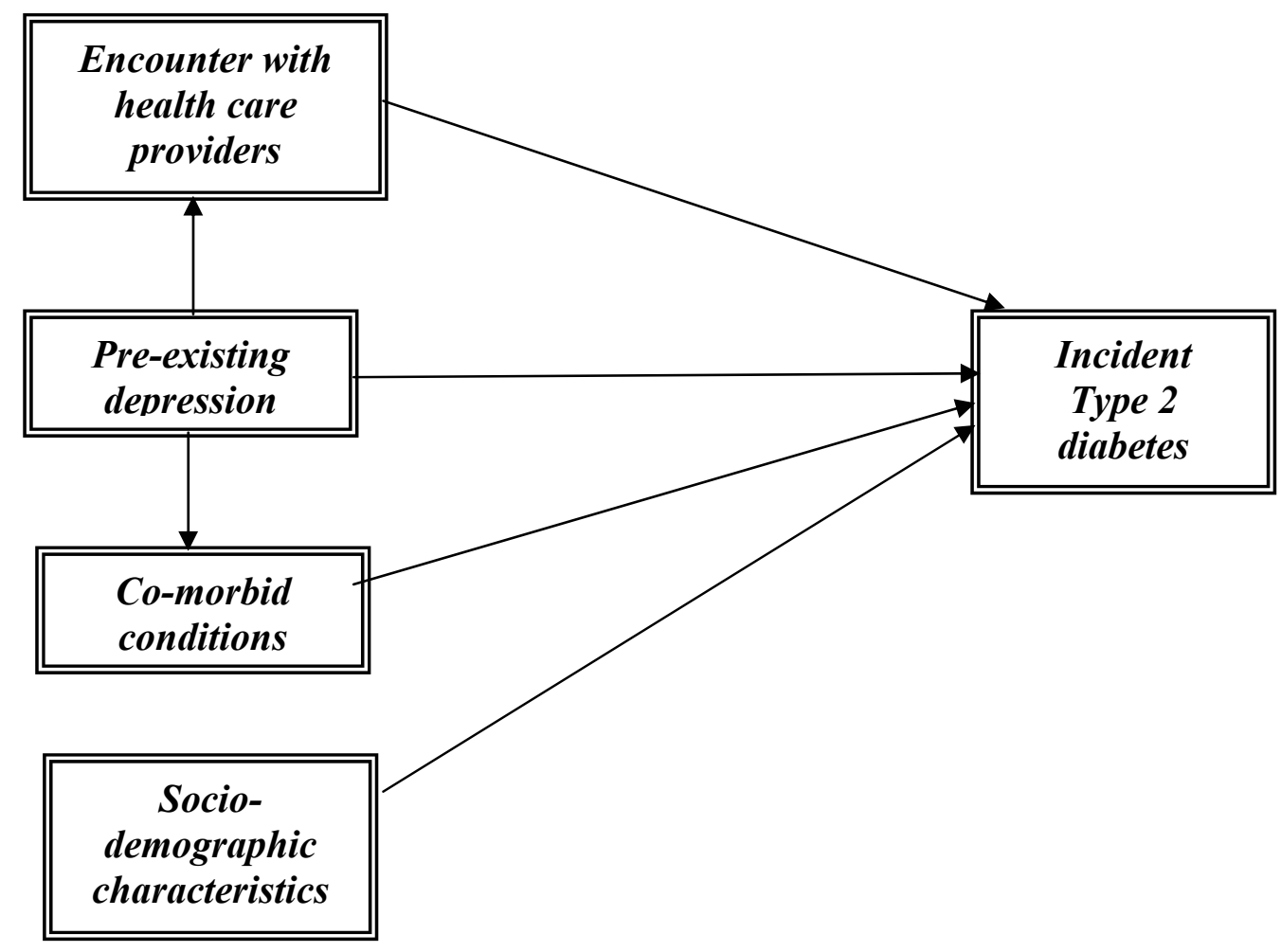


Patients with depression will be identified in the year 1997. As a comparison group, a group of Medicaid enrollees without depression will be identified in the year 1997. Patients with type 2 diabetes in the baseline year (i.e.1997) will be excluded from the analysis. This cohort of patients will be followed till December 31, 2002 and incident cases of type 2 diabetes will be identified in both the groups. The time frame for selection of patients and identification of incident cases for phase 1 of the study is shown in Figure 2. 
Figure 2: Time-line for Phase 1

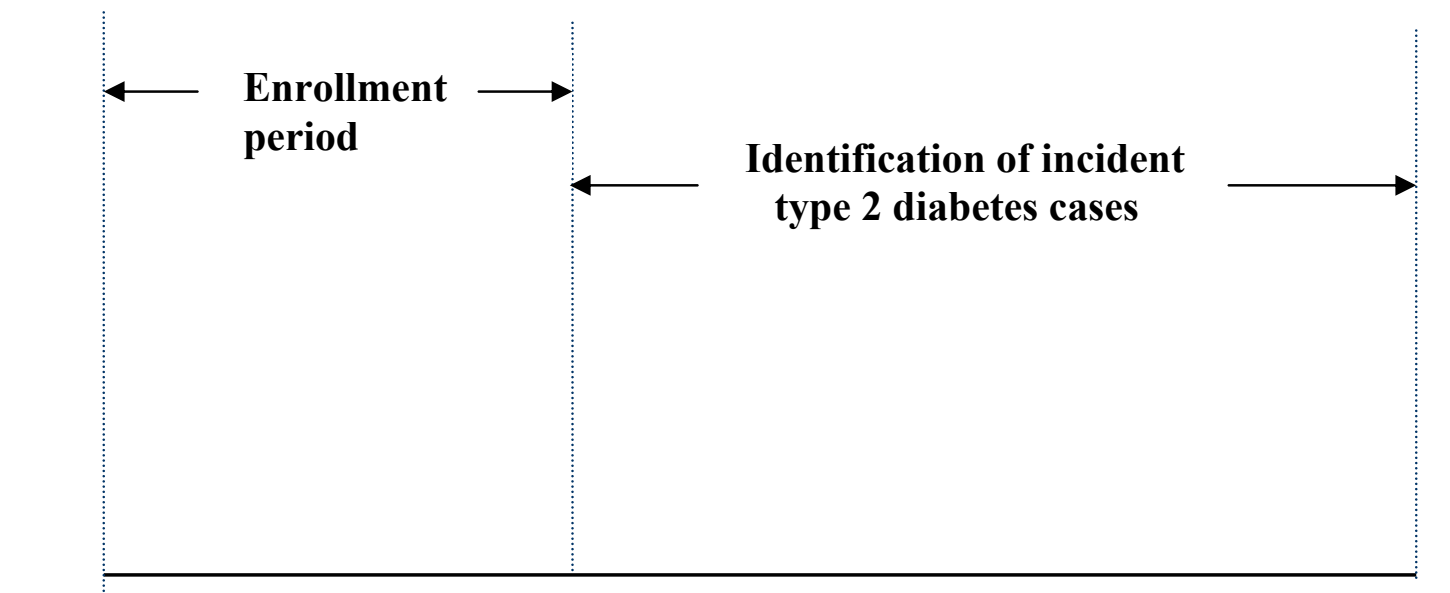

Jan 1, 1997

Jan 1, 1998

Dec 31, 2002 


\section{Phase 2}

As discussed in the introduction, depression may cause poor adherence to oral hypoglycemics. However, depression can affect type 2 diabetes outcomes in ways other than through its impact on adherence to oral hypoglycemic agents. ${ }^{39}$ Depression may oppose efforts to achieve appropriate glycemic levels via both behavioral and physiological pathways. Non-adherence with oral hypoglycemics might be just one of the behavioral mediators.

There is also the issue of aggravation of symptoms wherein patients with depression report significantly more physical symptoms on medical review of systems than non-distressed populations when controlling for severity of medical illness. ${ }^{40}$ Since co-morbid depression in diabetes is often associated with increased symptom reporting and amplification of symptoms, it is possible that physicians may order more testing and follow ups in these patients. ${ }^{41}$ This may explain findings, which have showed that diabetes patients with more severe depressive symptoms have significantly higher medical costs compared to non-depressed diabetes patients, even after controlling for severity of diabetes and other medical illness. ${ }^{28}$

In addition, metabolic alterations associated with depression may disrupt normal glucose metabolism. Studies suggest that depressive disorders lead to increased release of hormones such as cortisol, which increase insulin resistance and decrease glucose uptake. $^{42,43}$ Depression may also worsen diabetes outcomes through its effect on behavioral pathways. Depression is associated with increased smoking, alcohol consumption, unhealthy eating, and diminished physical activity. ${ }^{44,45}$ 
In view of the alternate theories of the impact of depression on diabetes, an important question that needs to be addressed is whether or not poor adherence actually mediates the relationship between depression and outcomes related to type 2 diabetes. Our approach to this question is to determine whether the association between depression and outcome is weakened after adjusting for level of adherence. The conceptual model in Figure 3 depicts the different factors in addition to pre-existing depression, which may impact adherence with oral hypoglycemics and outcomes related to type 2 diabetes.

These factors identified from the literature such as socio-demographic characteristics, comorbid conditions, severity of type 2 diabetes, patient-provider relationship, and complexity of regimen will be statistically controlled in the models to help in estimating the direct and indirect effects of pre-existing depression on outcomes related to management of incident type 2 diabetes. 
Figure 3: Conceptual framework for Phase 2

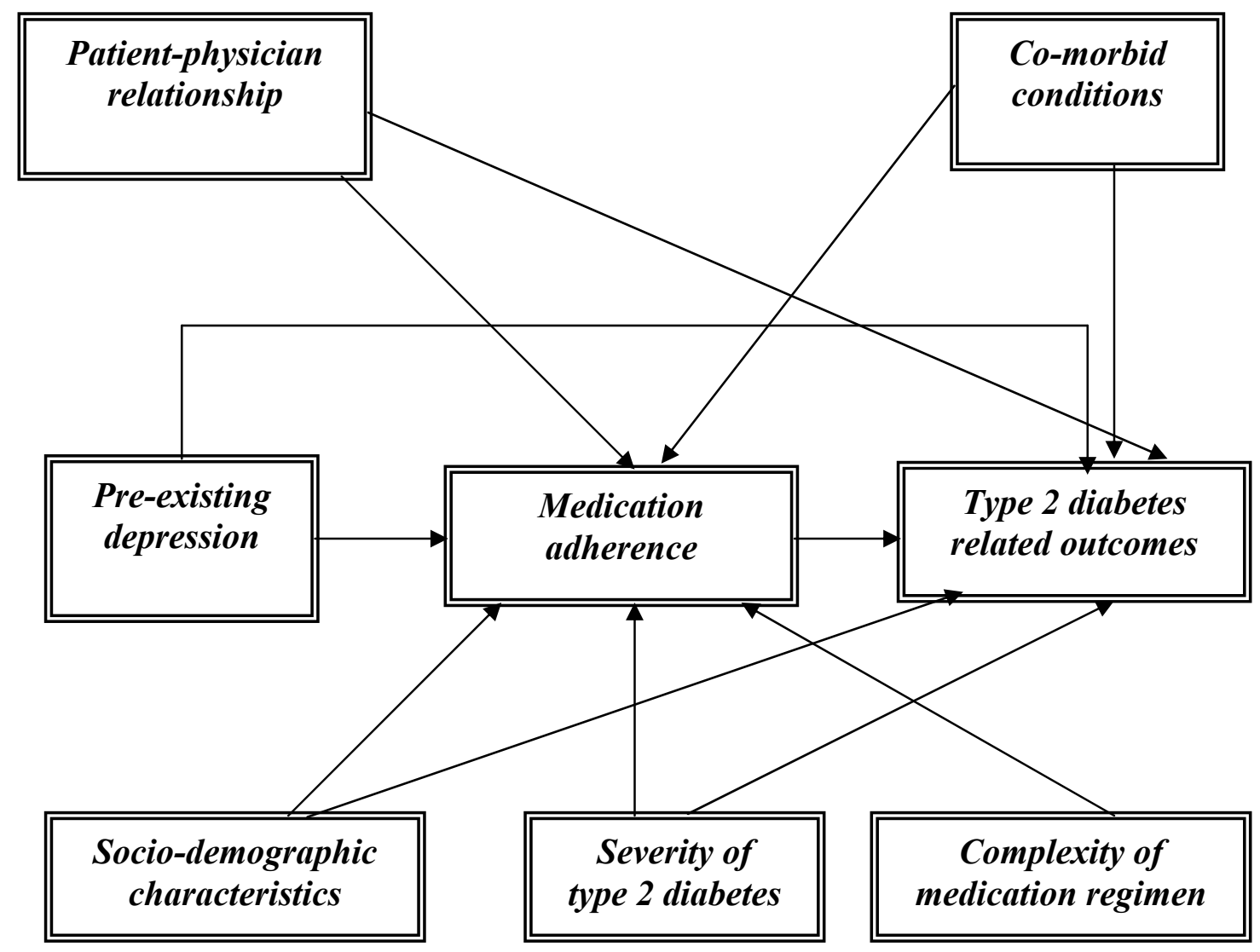


The study will examine the impact of preexisting depression on patterns of use of oral hypoglycemics, drug adherence and outcomes in patients with type 2 diabetes. Incident cases of type 2 diabetes will be identified between January 1, 1998 and December 31, 2000. A 12-month pre-period without a diagnosis for type 2 diabetes or a prescription for an oral hypoglycemic can confirm that the patient is newly diagnosed with type 2 diabetes. A 12-month pre-period will also be used to identify the diagnosis of depressive symptoms in these patients. Drug utilization patterns and adherence will be measured over the period of 12 months post index prescription. The study population will be limited to patients taking at least one oral agent for type 2 diabetes. Patients will have to be continuously eligible in both the pre and post periods. Incident type 2 diabetes patients who do not have preexisting depression but develop depression in the follow up period will also be excluded in the analysis. The time frame and design for enrollment and analysis for phase 2 is provided in Figure 4. 
Figure 4: Timeline for Phase 2

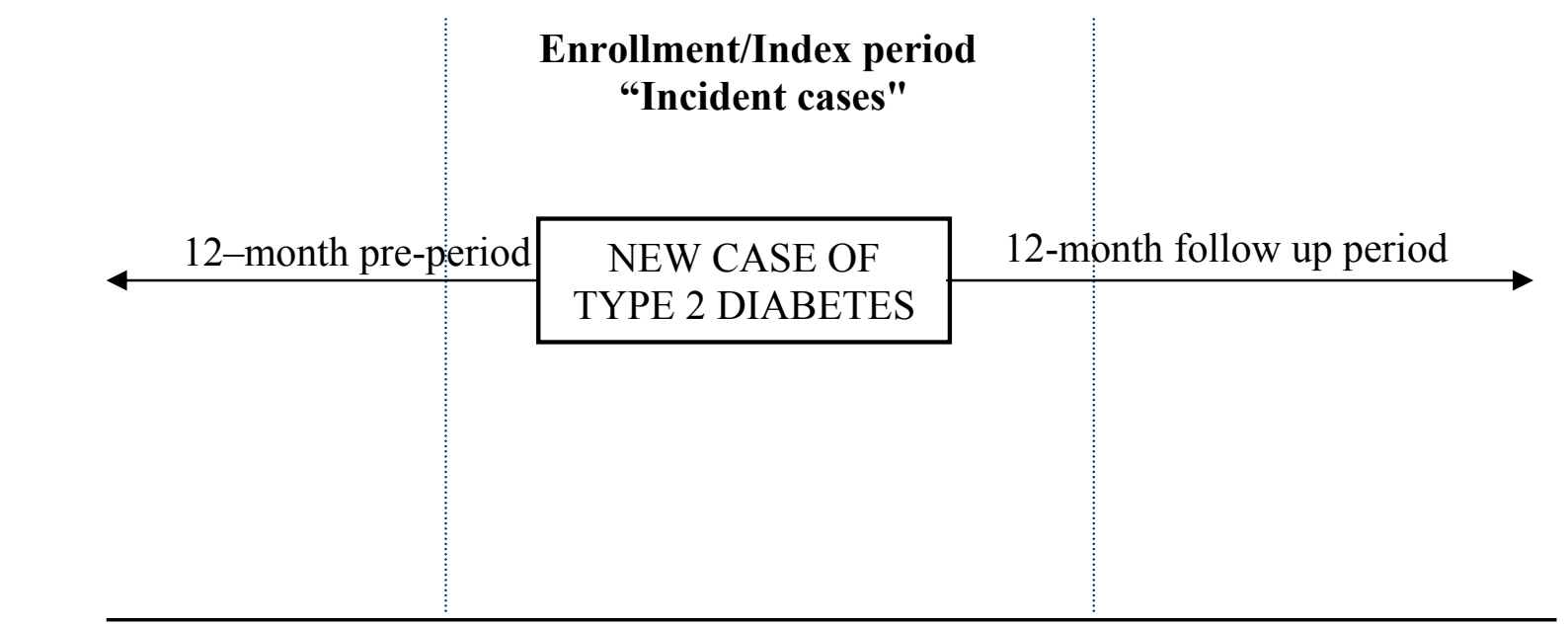

Jan 1, $1997 \quad$ Jan 1, 1998

Dec 31, 2000

Dec 31, 2002 


\section{Evaluation of Outcomes}

The study aims to examine the effect of pre-existing depression on adherence with oral hypoglycemics and subsequently the effect of outcomes related to management of type 2 diabetes. Thus, the selected cohort of newly diagnosed type 2 diabetes patients with and without pre-existing depression will be followed for a period of one year to detect the impact of depression on adherence with medications and other outcomes. Information on dosage, frequency, length of prescription, and number of tablets dispensed from the pharmacy claims of the patients will be used to assess refill information and compute patterns of oral hypoglycemic use and adherence indices. Examining the pattern of oral hypoglycemic use is especially important in patients newly diagnosed with type 2 diabetes. The following patterns will be examined:

- Augmentation: Augmentation refers to a situation where an initial regimen is modified by adding another agent of a different class or insulin.

- Switching: Switching refers to a situation when an initial regimen of one class of oral hypoglycemic agent is changed to another class, or insulin.

- Discontinuation: Patients discontinuing their oral hypoglycemic therapy

- Non-modification: Modification in medication regimen may indicate either augmentation or switching. Patients who experience no modification in their medication regimens for the entire follow up period (except discontinuers) will be designated as non-modifiers.

Sometimes advancement of type 2 diabetes is associated with a progressive loss of beta cell function and increased insulin resistance caused by persistent hyperglycemia and possible drug resistance. This is usually referred to as secondary failure wherein there 
is a loss of glycemic control in patients with type 2 diabetes whose disease has been initially well controlled with a single oral hypoglycemic agent. In our study, augmentation or switching will be used as proxies for secondary failure. In addition, survival analysis techniques will be used to estimate the impact of preexisting depression on time to secondary failure.

Another proxy of secondary failure is the switching of type 2 diabetes to insulin, as patients are typically switched to insulin therapy if they are on maximum oral hypoglycemics and still unable to control their blood glucose levels. Survival analysis techniques will also be used to estimate the impact of pre-existing depression on time to insulin switch.

Adherence indices will be derived separately for the different oral hypoglycemic agents used over the follow up period. Adherence will be modeled as the mean adherence for all antidiabetic drugs taken by each patient. Adherence to insulin will not be computed, as utilization of insulin through an administrative dataset is difficult to compute due to frequent changes in dosage instructions without proper documentation. Adherence indices such as the Medication Possession Ratio (MPR) will be used in the study.

The above adherence related outcomes could be termed as intermediate outcomes, which may in turn affect the final outcomes related to management of type 2 diabetes. Claims data can be used effectively to measure these final outcomes in terms of healthcare service utilization and costs for type 2 diabetes. Number of physician office visits, diabetes prescriptions, and Emergency room/hospitalization episodes specifically for type 2 diabetes will be measured. Overall type 2 diabetes costs and their breakdowns 
in terms of outpatient, inpatient, and pharmacy costs will also be computed. However, due to the nature of type 2 diabetes, it is highly likely that there is a high proportion of healthcare utilization and costs which is diabetes related but not directly attributable to type 2 diabetes. Hence, overall healthcare utilization and costs irrespective of diagnosis codes will also be computed. Appropriate econometric techniques will be used to estimate the impact of pre-existing depression on these intermediate and final outcomes controlling for the effect of factors mentioned in the conceptual model. In addition, the impact of preexisting depression on final outcomes directly and indirectly in the form of drug adherence as a mediating variable will be estimated using path analytic methods. 


\section{GOALS OF THE STUDY}

- Phase 1: To estimate the epidemiological burden (in terms of prevalence and incidence) of depression and type 2 diabetes.

- Phase 2: To estimate the impact of pre-existing depression on adherence with oral hypoglycemics and establish causal pathways for the impact of depression on outcomes related to management of type 2 diabetes.

\section{SPECIFIC OBJECTIVES}

\section{Phase 1}

Objective 1: To estimate the prevalence of co-morbid depression in patients with type 2 diabetes.

Null Hypothesis A: There is no difference between the prevalence of co-morbid depression in patients with type 2 diabetes and without type 2 diabetes.

Objective 2: To estimate the incidence of type 2 diabetes in patients with pre-existing depression.

Null Hypothesis B: There is no difference in the incidence rate of type 2 diabetes in patients with pre-existing depression as compared to patients without pre-existing depression. 


\section{Phase 2}

Objective 3: To examine the impact of pre-existing depression on patterns of oral hypoglycemic use in new patients with type 2 diabetes.

Null hypothesis $C$ : There is no difference in the pattern of oral hypoglycemic use between newly diagnosed type 2 diabetes patients who have pre-existing depression and those who do not have pre-existing depression.

Objective 4: To examine the impact of pre-existing depression on secondary failure with oral hypoglycemics.

Null Hypothesis D: There is no difference in the rate of secondary failure with oral hypoglycemics between newly diagnosed type 2 diabetes patients who have pre-existing depression and those who do not have pre-existing depression.

Objective 5: To estimate the impact of pre-existing depression on initiation of insulin therapy in patients with type 2 diabetes.

Null Hypothesis E: There is no difference in the rate of initiation of insulin therapy between newly diagnosed type 2 diabetes patients who have pre-existing depression and those who do not have pre-existing depression.

Objective 6: To estimate the impact of pre-existing depression on adherence to oral hypoglycemic agents. 
Null Hypothesis F: There is no difference in adherence to oral hypoglycemics between newly diagnosed type 2 diabetes patients who have pre-existing depression and those who do not have pre-existing depression.

Objective 7: To estimate the impact of pre-existing depression on type 2 diabetes related utilization and costs.

Null Hypothesis G: There is no difference in type 2 diabetes related utilization and costs between newly diagnosed type 2 diabetes patients who have pre-existing depression and those who do not have pre-existing depression.

Objective 8: To estimate the impact of pre-existing depression on overall health care utilization and costs.

Null Hypothesis H: There is no difference in overall health care utilization and costs between newly diagnosed type 2 diabetes patients who have pre-existing depression and those who do not have pre-existing depression.

Objective 9: To examine the causal pathways between pre-existing depression, adherence, and outcomes such as resource utilization and costs for type 2 diabetes. Null Hypothesis I: Adherence to oral hypoglycemics is not a mediating variable between the presence of pre-existing depression and outcomes related to management of type 2 diabetes. 


\section{SIGNIFICANCE OF THE STUDY}

The results of this study can help prioritize risk factors for disorders perhaps leading to efforts of prevention. The results might indicate a benefit to screen a select population for adults with diabetes. This is important as screening of all primary care patients is not shown to be cost-effective and screening hence should be restricted to only a high-risk population. Also, if the results indicate poorer outcomes and higher costs of diabetes management in patients with co-morbid depression then early detection and treatment of depression could potentially decrease total cost of diabetes care.

If a relationship between adherence and depression is demonstrated it may explain the high morbidity and mortality rates in depressed patients. It would also call for improving the detection and treatment of depression. Although a majority of the depressed patients present in primary care clinics, there is substantial evidence that depression is under detected and inadequately treated in such settings. ${ }^{46,47}$ Increased efforts to detect and treat depression may be associated with better glycemic control. For patients who are beginning their courses of treatment for chronic diseases, screening for depression might prove to be a useful identifier of possible future non-adherence and might suggest closer monitoring and assistance to achieve adherence. On the other hand, clear non-adherence with a specified treatment regimen should raise suspicion of comorbid depression. Although it remains to be determined whether treating depression will result in improved patient adherence, the recognition of depression as a significant risk factor for non-adherence with medical treatment carries the potential to improve medical practice, reduce patient disability, enhance patient functioning, and improve outcomes. 
Research indicating that depression is associated with health outcomes and additive medical costs in specific chronic illness such as diabetes may lead to changes in guidelines for diabetes management and encourage national agencies and Health Maintenance Organizations (HMOs) to screen for depression in patients with such chronic conditions and to improve the clinical management of depression. 


\section{LIMITATIONS}

Inherent limitations of claims database are applicable to the study as well. ${ }^{36}$ Measuring adherence using prescription refills fails to assess the timing and quantity of medication taken. Many patients request refills regularly when reminded, even if they have not run out of drugs and some others stockpile medications or have quantities of medications in several areas for convenience. In addition, Medicaid enrollees may lack the economic disincentives to purchase only the medications that they will consume. However, one can assume that refilling a prescription might be a good indicator of willingness to adhere to medications. Also, as in a population enrolled in Medicaid procurement of drugs from other sources is highly unlikely, a prescription which is not refilled can be considered a good indicator of a prescription not taken and thus of nonadherence. It is also not possible to verify whether the patient discontinued taking the medication because of lack of efficacy, adequate control through other methods, or change in therapy by physician. One also misses the most non-adherent patient, i.e. those who do not fill any oral hypoglycemic medications.

Adherence to medications can be affected by a lot of factors, which will not be measured in this study such as social support, perceived risk of the outcomes of being non-adherent, and others. Also, severity of disease is an important predictor of adherent behavior. $^{48,49}$ As clinical information is not available, our measures of disease severities are proxies and could be subject to measurement bias. Lack of clinical information also prevents us from estimating the impact of adherence on clinical parameters such as blood glucose or A1C levels. In addition to adherence with oral hypoglycemics, diabetes control is also affected by factors such as adherence to specified diet and exercise 
regimen. Although one can make an assumption that a patient's adherence to oral hypoglycemics can be a valid proxy of adherence to other important self-management behaviors such as diet and exercise, studies in literature have demonstrated low correlations between these..$^{50,51}$

Another major limitation of the study is the issue of undiagnosed/unrecognized depression. ${ }^{46}$ This can potentially lead to misclassification bias as patient classified as non-depressed diabetics could be patients with undiagnosed depression and this could lead to underestimation of the effect of depression on adherence to medications and related outcomes. However, one can easily assume that diagnosed patients will have more severe depressive symptoms as compared to an undiagnosed population. An alternative would be to identify depression in patients through administration of a self reported diagnostic measure. However, self reported depression has been consistently shown to be highly unreliable and especially in patients with chronic conditions such as diabetes, the severe symptoms of diabetes may be misattributed to depression. Depressive symptom inventories such as CES-D are found to be overly sensitive to somatic complaints that may be the result of diabetes rather than depressed mood. Also, self-reported measures may identify a broader spectrum of depression disorders (e.g dysthymic disorder or minor or subsyndromal depression) or symptoms that reflect comorbid psychiatric illness (e.g anxiety or substance abuse disorders) or general distress. $^{52,53}$ 


\section{CHAPTER TWO}

\section{LITERATURE REVIEW}

\section{Overview of Type 2 Diabetes}

Diabetes is a highly prevalent physiological condition involving high blood glucose levels. There are two classifications of diabetes, type 1 or insulin dependent diabetes mellitus, and type 2 diabetes or non-insulin dependent diabetes mellitus. ${ }^{54}$ Type 1 diabetes affects approximately $10 \%$ of the diabetic population in the United States. ${ }^{55}$ It is a disease of the beta cells of the pancreatic islets, destroyed by an immunologically mediated inflammatory reaction. In type 1 diabetes, the pancreas loses its ability to produce any insulin, resulting in uncontrolled elevations of blood glucose. Although both type 1 and type 2 diabetes share the common feature of elevated blood glucose levels, type 2 diabetes typically involves the following three stages in most patients. The first stage involves the principal underlying defect of insulin resistance. Although insulin can attach normally to receptors on liver and muscle cells, certain mechanisms prevent insulin from moving glucose into these cells. The ability of beta cells of the pancreas to secrete insulin is not affected. However, as the condition progresses there is subsequent beta cell exhaustion and secretion of insulin is affected. Eventually, the cycle of elevated glucose further impairs and possibly destroys beta cells, thereby stopping insulin production and causing full-blown diabetes. ${ }^{56-58}$

Genetic factors play an important role with regard to both insulin resistance and impaired beta cell function. However, environmental factors such as high calorie intake and limited physical activity also play a major role in the incidence and progression of 
type 2 diabetes. Type 2 diabetes patients initially have mild symptoms and after years of disease may present with complications of diabetes. Symptoms of type 2 diabetes that are typically seen at diagnosis may be thirst, increased urination, fatigue, and blurred vision. However, most of the diagnosis is incidental, and up to $50 \%$ of type 2 diabetes patients are relatively asymptomatic at diagnosis. ${ }^{55}$ However, all type 2 diabetes patients are at risk of severe complications as the condition progresses. The life expectancy of patients with type 2 diabetes is reduced by $30-40 \%$ for those in the age range of $40-70$ years. ${ }^{59}$ Most of this mortality and morbidity associated with type 2 diabetes can be attributed to diabetic complications, which can be divided into the following 2 categories:

- Microvascular complications: These complications affect the smaller blood vessels and are specific complications of diabetes and do not occur in patients without diabetes. Microvascular complications can occur in different body sites such as the eye (retinopathy), the kidney (nephropathy), and the nervous system (neuropathy). These complications can lead to debilitating conditions such as blindness, renal failure, and foot problems leading to amputations. ${ }^{60}$

- Macrovascular complications: These complications are due to the effect of high glucose levels on larger blood vessels and are not unique to diabetic patients but occur to a greater extent in diabetic patients. Macrovascular disorders include conditions such as cardiovascular disease, cerebrovascular disease, and peripheral vascular diseases..$^{60,61}$ 


\section{Diagnostic Tests for Type 2 Diabetes}

Random plasma glucose or fasting plasma glucose levels are typically used for diagnosis of diabetes. Measurement of fasting plasma glucose requires the patient to fast overnight for at least 8 hours. Another test for diagnosis of diabetes, which is not used as frequently in clinical practice, is the oral glucose tolerance test. ${ }^{62}$ The American Diabetes Association (ADA) currently recommends measurement of glycosylated hemoglobin (A1C) for monitoring of glycemic control in patients and it is widely used by clinicians both for monitoring and diagnostic purposes. ${ }^{63}$ A1C levels yield a measure of chronic glycemia from the slow, posttranslational non-enzymatic glycation of hemoglobin. A1C provides a moving average of blood glucose levels integrated over time, weighted proportionally toward recent levels. Clinical studies have shown a strong correlation between $\mathrm{A} 1 \mathrm{C}$ levels and the mean level of blood glucose over the preceding one to three months.

\section{Treatment Options}

Type 2 diabetes is a progressive disease and is typically managed using a "stepped care approach". ${ }^{64,65}$ Patients are initially managed with non-pharmacological options such as diet and exercise. As the disease progresses, oral pharmacological agents are used to control blood glucose levels. Typically, these agents maintain blood glucose levels, and some patients can be maintained on single oral agents for a few years. However, patients eventually show secondary failure to their treatment regimen, requiring therapy with a combination of oral hypoglycemics. While combination therapies lead to better glycemic control, they can also potentially increase the risk of 
side effects of therapy. Once oral combination therapies fail, patients progress to insulin therapy.

\section{Non-pharmacological therapy}

Diet, exercise, and weight loss are the initial treatment modalities in the "stepped care" approach for type 2 diabetes. These lifestyle behavioral changes not only lower blood glucose levels, but also help in controlling co-existing risk factors for future complications. In a meta-analysis of 14 studies $^{66}$ examining interventions to increase physical activity among diabetes patients, individuals receiving these interventions had a mean post study A1C level of $7.7 \%$ compared with $8.3 \%$ among comparison group patients. In addition to affecting glycemic control, moderate physical activity improved other cardiovascular risk factors such as high-density lipoprotein cholesterol and blood pressure. Unfortunately, most patients are unable to achieve adequate control with lifestyle changes alone. However, they can play a pivotal role in diabetes management in conjunction with medical regimen. A controlled diet and regular physical activity are therefore, recommended for a majority of patients with type 2 diabetes especially those who are overweight. ${ }^{67,68}$

\section{Oral hypoglycemic agents}

A variety of oral medications for type 2 diabetes with different mechanism of actions are available. They are typically divided into distinct classes based on their mechanism of action. ${ }^{69-73}$

Sulfonylureas: Sulfonylureas have been available in the United States since 1954. They are further classified into: 
First generation sulfonylureas: including drugs such as chlorpropamide, tolbutamide, acetohexamide, and tolazamide.

Second generation sulfonylureas: including drugs such as glyburide, glipizide, and glimepiride. Second generation sulfonylureas are more potent and probably safer than first generation sulfonylureas but essentially of equal efficacy.

Mechanism of action: Sulfonylureas interact with the pancreatic beta cells leading to a closure of voltage-dependent potassium adenotriphosphate channels facilitating cell membrane depolarization, calcium entry into the cell, and insulin secretion. Thus, sulfonylureas allow for insulin release at lower glucose thresholds than normal. Side effects: Sulfonylureas are associated with weight gain, typically from five to ten pounds, problematic in patients already obese at diagnosis. Sulfonylureas also cause hypoglycemia, especially in elderly, those with worsening renal functioning, and those with irregular meal schedules.

Indications: Sulfonylureas are approved for use as monotherapy and in combination with all other oral agent classes (except the non-sulfonylurea secretagogues) and insulin.

Meglitinides: Meglitinides were introduced in the United States in 1998 and comprise a new class of insulin secretagogues derived from benzoic acid that are structurally and pharmacologically distinct from oral sulfonylureas. This class includes drugs such as repaglinide, nateglinide, and mitiglinide.

Mechanism of action: They are also known as non-sulfonylurea insulin secretagogues as their mechanism of action is similar to that of sulfonylureas: interaction with voltage dependent potassium adenotriphosphate channels on beta cells. They are different from 
sulfonylureas in having short metabolic half-lives, which result in brief episodic stimulation of insulin secretion. Thus these newer agents are rapidly metabolized and shorter acting and better than sulfonylureas in controlling elevation of glucose levels after meals. Also, since less insulin is secreted several hours after the meal, there is decreased risk of hypoglycemia during this late postprandial phase.

Side effects: Side effects include diarrhea and headache. Some of these agents such as repaglinides may pose a slightly increased risk for cardiac events.

Indications: They are approved for use either as monotherapy or in combination with metformin.

Biguanides: Although available internationally for decades, metformin, a biguanide was released in the United States only in 1995.

Mechanism of action: It reduces glucose production in the liver and increases tissue sensitivity to available insulin. These agents may have a beneficial effect on cholesterol, blood pressure, and clotting factors. Metformin does not cause hypoglycemia or weight gain and hence is particularly well suited for obese type 2 diabetes patients.

Side effects: More than $30 \%$ of metformin users experience gastrointestinal problems including nausea and diarrhea. Some patients may have excessive weight loss. Rare but life threatening lactic acidosis is seen primarily in persons with renal insufficiency, which impairs the clearance of metformin.

Indications: Approved for use as monotherapy and in combination with sulfonylureas and other secretagogues, thiazolidenediones, and insulin. 
Thiazolidenediones: In 1997, troglitazone was the first thiazolidenedione that was introduced in the United States but was withdrawn after reports of liver toxicity in March of 2000. The other agents in this class, which are currently used, are pioglitazone and rosiglitazone.

Mechanism of action: Thiazaolidenediones reduce insulin resistance by activating certain genes involved in fat synthesis and carbohydrate metabolism. These agents all improve cholesterol levels, including High Density Lipoprotein (HDL) levels and also may reduce the risk for blood clots. Thiazolidenediones have a relatively slow onset of action and lower the glucose level progressively over 16 weeks.

Side effects: Troglitazone (rezulin) was removed from the market owing to its hepatotoxicity. Although the other thiazolidenediones do not cause liver toxicity, they are associated with side effects such as weight gain, which can be as great or greater than that with sulfonylureas. Some patients may also experience anemia and edema. Indications: Thiazolidenediones are the most expensive class of oral hypoglycemics and are indicated as monotherapy and in combination with metformin, sulfonylurea, and insulin (pioglitazone only).

Alpha-glucosidase inhibitors: Includes drugs such as acarbose (Precose, Glucobay) and miglitol (Glyset). These were introduced in 1996.

Mechanism of action: Alpha-glucosidase inhibitors act by a unique mechanism of delaying intestinal absorption of carbohydrates. Although their benefit in monotherapy and combination therapy has been proved, the incremental reduction in $\mathrm{A} 1 \mathrm{C}$ is relatively modest. Their greatest effect is on postprandial glucose levels; whereas, the effect on 
fasting blood glucose levels is small. These agents are non-systematic and hence do not cause hypoglycemia and weight gain.

Side effects: The most common side effects are gastrointestinal side effects such as flatulence, bloating, and diarrhea.

Indications: They are approved for use as monotherapy and in combination with sulfonylureas. However, they are rarely used as monotherapy because of their mild efficacy.

As mentioned previously, initial therapy with oral hypoglycemics fails to control blood glucose indefinitely. Results from the UKPDS indicated that after a period of 3 years, only $50 \%$ of type 2 diabetes patients were adequately controlled with a single drug, and after nine years this percentage had decreased to $25 \%{ }^{5}$ A second oral agent of a different class is commonly added when initial glucose control begins to fail. Combination of these agents are often used to increase their effectiveness and are becoming increasing popular for use before a patient's transition to insulin therapy. However, as failure of oral therapy typically coincides with beta cell exhaustion, insulin use eventually is required in most patients with type 2 diabetes. 


\section{Guidelines for Therapy}

Recent guidelines have stressed the importance of strict glucose control in patients with type 2 diabetes. ${ }^{6,74}$ The UKPDS has shown that for every $1 \%$ decrease in A1C levels, future micro-vascular complications decrease by $35 \% .^{5}$ The goals of treatment of type 2 diabetes are to reduce the symptoms of hyperglycemia and to prevent acute and chronic complications of the disease.

Metformin and sulfonylureas are the mainstay of oral hypoglycemic therapy for type 2 diabetes. $^{75}$ As a high fraction of type 2 diabetes patients in the United States are obese, metformin is typically the favored initial therapy. ${ }^{76}$ For the obese patients in whom insulin resistance is a major factor, thiazolidenediones are a suitable alternative when metformin is not tolerated or is contraindicated because of renal insufficiency. Sulfonylureas are often considered the first choice for patients who are not significantly overweight at diagnosis. $^{72}$

The initial treatment selection for type 2 diabetes depends on the severity of symptoms and the degree of elevation of glucose..$^{70,71}$

- Patients presenting with marked symptoms and acute hyperglycemia with Fasting Plasma Glucose (FPG) levels greater than $300 \mathrm{mg} / \mathrm{dl}$ are most quickly and effectively treated with insulin. With reduction of glucotoxicity and institution of a diet and exercise program, insulin therapy can often be withdrawn with maintenance of good glycemic control. Metformin is particularly to be avoided in these patients because of the risk of lactic acidosis.

- Patients with FPG levels of 200 to $300 \mathrm{mg} / \mathrm{dl}$ fall in an intermediate group. One option is to treat them with diet and exercise alone with close-follow up. Failure 
to respond to non-pharmacological methods should quickly lead to initiation of oral agent monotherapy. If the patient is symptomatic, starting immediately with diet, exercise, and oral agent monotherapy is most often a successful and better strategy.

- Patients with FPG levels of less than $200 \mathrm{mg} / \mathrm{dl}$ usually can be treated successfully with diet and exercise initially. Although diet and exercise eventually lose effectiveness as the sole therapy, reinforcing the importance of diet and exercise generally improves the response to oral hypoglycemics.

\section{Treatment Goals}

The American Diabetes Association (ADA) recommended targets for glycemic control include a pre-prandial blood glucose level of 80 to $120 \mathrm{mg} / \mathrm{dl}$, a bedtime blood glucose level of 100 to $140 \mathrm{mg} / \mathrm{dl}$, and an $\mathrm{A} 1 \mathrm{C}$ level of less than $7 \%$. Change in therapy or additional intervention is typically advised at an A1C level of $8 \%$ or more. ${ }^{6}$ However, more stringent guidelines have been offered by the American College of Endocrinology and the American Association of Clinical Endocrinologists: pre-prandial blood glucose levels less than $110 \mathrm{mg} / \mathrm{dl}$, two-hour post-prandial glucose levels less than $140 \mathrm{mg} / \mathrm{dl}$, and A1C levels at $6.5 \%{ }^{74}$

All these recommendations are based on three landmark studies: Wisconsin

Epidemiological Study of Diabetic Retinopathy, ${ }^{60}$ the Kumamoto study, ${ }^{77}$ and the UKPDS, ${ }^{5}$ which have shown unequivocally that maintaining blood glucose concentrations as close to normal decreases the incidence of diabetic complications. 


\section{Impact of Glycemic Control on Type 2 Diabetes Outcomes}

Epidemiological data from the Wisconsin Epidemiological Study of Diabetic Retinopathy ${ }^{60}$ firmly implicate glycemic control in the development of the microvascular complications associated with type 2 diabetes. In this study baseline A1C levels were related to incidence and progression of retinopathy, neuropathy, and nephropathy. Benefits of glycemic control on microvascular complications have also been demonstrated by a few other randomized trials. The Kumamoto trial ${ }^{77}$ was conducted in a relatively small sample of 110 lean Japanese patients with type 2 diabetes randomized to conventional or intensified insulin therapy. Intensive therapy was associated with a lower incidence of macrovascular events ( 0.6 versus 1.3 events per 100 patient-years). More recently, the results of the large UKPDS ${ }^{5}$ have confirmed that improved glycemic control is associated with reduced rates of microvascular complications. However, the UKPDS did not confirm such a beneficial effect on macrovascular disease. The UKPDS has the advantage over the Kumamoto trial of including typical overweight and obese patients who may also have had lesser degrees of insulin deficiency.

The UKPDS and Kumamoto trials clearly showed that the incidence of complications dramatically decreased at approximately five to ten years from initiation of intensive glycemic control. An economic analysis of the UKPDS ${ }^{78}$ found that increased therapy costs of intensive glycemic control in type 2 diabetes patients are offset by significantly reduced costs of complications. The net cost of intensive blood glucose control was recently estimated at 1,435 pounds per patient annually, the cost saved on complications at approximately 15,000 pounds and the cost per event free year of intensive blood glucose control at about 1,166 pounds. Within the metformin sub study 
of the UKPDS, another pharmacoeconomic study determined that intensive management of the overweight was a more cost-effective option. ${ }^{79}$ Thus, improving long-term glycemic control should reduce the costs of treating diabetes-related complications. These savings would more than offset the costs of interventions required to achieve glycemic control.

A number of recent studies have shown that the economic benefits of improved glycemic control accumulate much sooner than the seven-ten year trial period examined in the UKPDS. A study conducted by Testa and colleagues demonstrated evidence of short-term cost savings. They compared short-term effects on symptoms, quality of life, work productivity, and health care use of active hypoglycemic therapy versus placebo in a randomized trial and found that at 15 weeks, patients who controlled their glycemic levels reported better health and work productivity and less use of health care services. The improvements in quality of life were both consistent and substantial across all domains and were partially due to the reduction in adverse events associated with hyperglycemia. The study results demonstrated that improved glycemic control (1.8 percentage points lower A1C levels) resulted in increased productivity (12\%) within a period of 12 weeks. ${ }^{80}$ In a study conducted by Gilmer and associates in a large HMO over the period of four years, a strong association was demonstrated between baseline glycemic levels in 1992 and subsequent health care expenditures over the next three years. Lower baseline levels of A1C were associated with significantly lower charges for care and after adjusting for age, sex, and co-morbidity, higher baseline levels of A1C were associated with significantly higher subsequent chares for care. Their model suggested health care savings ranging from $\$ 400$ to $\$ 4000$ per patient over a three-year 
period, with savings increasing depending on the level of baseline A1C. ${ }^{81}$ Wagner and colleagues at Group Health Cooperative of Puget Sound demonstrated reductions in health care utilization and costs within one to two years of improvement of A1C levels. In a retrospective analysis, better glycemic control (reduction and maintenance of less than or equal to one percentage point in A1C level) resulted in average cost savings for the HMO of $\$ 685$ to $\$ 950$ per patient per year between 1994 and 1997 . The lower end of the scale corresponded to patients with uncomplicated diabetes whereas the higher end reflected complicated diabetes. ${ }^{82}$

\section{Impact of Adherence with Oral Hypoglycemics on Glycemic Control and Type 2}

\section{Diabetes Outcomes}

A number of studies in the literature have empirically evaluated the association between medication adherence and diabetes metabolic control. Diehl and colleagues ${ }^{8}$ used pill counts to assess adherence in 77 patients with diabetes and found a trend towards higher fasting blood glucose levels in those taking less than $80 \%$ of prescribed dosages. Chousa and associates ${ }^{9}$ similarly found adherence assessed by pill count to be associated with metabolic control measured by A1C among 107 patients with type 2 diabetes. Using self-reported adherence plus the pharmacy records of 65 patients with type 2 diabetes, Peterson and colleagues ${ }^{10}$ also demonstrated an association between adherence and a composite measure of metabolic control, including A1C. However, most of these studies relied on small patient samples and self-report as the measure of drug adherence. A recent study conducted by Schectman and associates ${ }^{11}$ examined this issue in a sample of 810 type 2 diabetes patients receiving medical care from a university based internal medicine clinic serving low-income population in rural central Virginia. 
The study used refill data to establish adherence with oral hypoglycemics and found that better metabolic control was associated with better medication adherence. For each $10 \%$ increment in drug adherence, A1C significantly decreased by $0.16 \%$. These results were duplicated in a prospective assessment of self-reported adherence in a homogenous cohort of 11,896 type 2 diabetes patients in Europe. The study results indicated that A1C levels were associated with adherence to medications, with a $1.4 \%$ mean difference between groups with optimal and worst adherence. ${ }^{83}$

However, conflicting results are present for the effect of medication adherence on utilization and costs associated with type 2 diabetes. In a retrospective cohort study conducted in a non-managed care setting, a threshold effect was observed where a target level of adherence was needed before medical care costs were reduced. Increased pharmaceutical adherence was associated with fewer emergency department visits and inpatient admissions, suggesting improved disease control. Similar costs and utilization patterns were evident for both overall health care and diabetes-related care. The impact of medication adherence on decreasing the use and cost of non-pharmacy services was observed at a threshold of 20 to $39 \%$ adherence. However, medication adherence was not associated with decreased overall healthcare costs because medication costs offset medical care costs savings. ${ }^{84}$ In another study, which examined a longitudinal cohort of older adults in the southeastern United States, increased adherence with diabetes medications was found to be the strongest predictor of overall health care costs. Results indicated a statistically significant 8.65 to $28.9 \%$ decrease in annual costs with every $10 \%$ increase in medication adherence. ${ }^{85}$ 
Non-adherence to oral hypoglycemic agents can also result in dire consequences for patients with type 2 diabetes. A review of 13,309 patients with type 2 diabetes from the UK Mediphys database documented patient visits to primary care physicians, prescription refills, and emergency events between 1991 and 1997. Patients who discontinued their oral hypoglycemics were approximately twice as likely to experience an emergency medical event and had a mortality rate that was three times that of continuers. ${ }^{13}$ Thus, adherence to oral hypoglycemics assumes great importance in preventing future complications and controlling health care expenditures in patients with type 2 diabetes.

\section{Adherence to Oral Hypoglycemics in Patients with Type 2 Diabetes}

On the basis of the discussion in the sections above, adherence to oral hypoglycemics assumes great importance in diabetes management. However, medication non-adherence is a major problem in all therapeutic areas with estimates of nonadherence rates ranging from $30 \%$ to $60 \%$ with higher rates in symptom free patients. ${ }^{86}$ A similar trend is seen in patients with type 2 diabetes especially those who are newly diagnosed with the condition. A study conducted in Tayside, Scotland of 2,920 type 2 diabetes patients who received a prescription for oral hypoglycemic drugs for at least 12 months between January 1993 and December 1995 showed that adherence to these agents was suboptimal, with only about one-third of those treated with oral hypoglycemic obtaining insufficient drug supplies for "adequate" drug coverage (defined as 90\%). ${ }^{17}$ These results are consistent with a recent observational study of subjects enrolled in a health maintenance organization that suggested that $31 \%$ of those receiving sulfonylureas alone did not purchase any drug in the following year. ${ }^{15}$ A similar study conducted by 
Skaer and associates estimated that $10-30 \%$ of patients with type 2 diabetes withdraw from their prescribed regimen within one year of diagnosis and of the remainder; nearly $20 \%$ administer insufficient medication to facilitate an adequate reduction in blood glucose. ${ }^{14}$

A recent analysis of a large administrative pharmacy claims database found persistence to oral hypoglycemic therapy over a 12-month period to be low, ranging from $31 \%$ for alpha glucosidase inhibitors to $60 \%$ for metformin, with adherence rates of 70 $83 \%$. The study results also indicated that the addition of a second agent was observed in $15 \%$ of the population with a mean time to augment therapy being approximately four months. When a composite measure of change in therapy including switching or augmentation was used, a striking $36 \%$ of the patients were found to change therapy over a 12-month follow up period. ${ }^{16}$ Similar studies have found even lower adherence rates, although these differences can be explained by methodological and population differences. These studies used refill data for measuring adherence where one assumes that every tablet dispensed is actually taken by the patient. Thus, these adherence rates may be overestimates, thus providing further evidence that adherence to diabetic regimen is a cause for concern and an important challenge for health care professionals and patients with type 2 diabetes.

\section{Factors Affecting Adherence}

The nature and determinants of non-adherent behavior are complex and not yet well understood. It is commonly believed that adherent behavior is a multidimensional issue, which needs to be defined for each population and disease. The four dimensions for determination of adherence identified by most researchers are: treatment regimen 
complexities, health status and disease-experience factors, characteristics of the interaction with healthcare providers, and patient related attributes. ${ }^{18}$

\section{Regimen factors}

Regimen factors greatly affect adherence. Frequency of dosing and the number of medications (complexity of regimen) have been shown to reduce adherence. ${ }^{20}$ Other medication related characteristics such as route of administration could also impact adherence. Dezii and colleagues ${ }^{87}$ evaluated differences in adherence and persistence with prescribed therapy of once-daily (OD) dosing compared with twice-daily (BID) dosing of glipizide in patients with type 2 diabetes from a pharmacy benefit manager claims database. Patients new to extended-release gastrointestinal therapeutic system (GITS) and immediate-release glipizide therapy were identified and followed for one year. The authors concluded that initiation of OD pharmacotherapy results in better adherence and persistence compared with a BID regimen, despite a greater daily pill burden in the OD cohort. These data suggest that dosing frequency exerts a greater impact on patient adherence and persistence than number of tablets per dose. Schectman and associates ${ }^{11}$ saw similar results of improved adherence in patients prescribed OD dosing as opposed to multiple dosing in the study.

Conflicting results have been found for the association between number of drugs prescribed and mean adherence. Many studies have reported a positive association between number of drugs prescribed and mean adherence. ${ }^{88}$ It is possible that patients on more medications are more likely to adhere because of greater disease severity, concern, or knowledge, and a more established regimen. However, Schectman and associates ${ }^{89}$ reported contrasting finding of an inverse association between number of drugs 
prescribed and minimum adherence levels suggesting lower adherence to individual drugs in a complex regimen. Similar results were found in a retrospective study by Dailey and associates, ${ }^{90}$ comparing patterns of use and persistence in patients with diabetes initiated on monotherapy versus those on polytherapy. For the patients who had no modification of their medication regimen, persistence with sulfonylurea or metformin monotherapy was $65 \%$ greater than with polytherapy over a one-year period. Similar results were seen for adherence with oral hypoglycemics, wherein adherence with sulfonylurea or metformin monotherapy was $45 \%$ greater than with polytherapy.

\section{$\underline{\text { Relationship factors }}$}

Patients may not fear possible medication side effects or drug interaction if they understand their prescriptions and have an open, communicative relationship with their health care professional. Researchers have shown that satisfaction with the interpersonal quality of the patient provider relationship is significantly associated with adherence to

diabetes therapy. ${ }^{91,92}$ Anderson and colleagues ${ }^{93}$ state that physicians must be aware of the likelihood of non-adherence in individual patients and make efforts to persuade patients of the importance of adherence to a program designed to reach and maintain therapeutic goals. It is the physician's responsibility to teach, motivate, and strengthen the patient to maximize adherence as part of a "therapeutic partnership".

\section{Health status and disease experience}

Anderson and Kirk $^{93}$ suggested that if an illness has easily recognizable and unpleasant symptoms that are improved by following treatment recommendation, adherence is more likely. In diabetes, many of the symptoms are not evident until later in life. Therefore, many individuals will not feel the urgency of undergoing a treatment 
regimen or making lifestyle changes immediately. Adherence is also more likely if the patient has experienced the illness previously or has known someone impaired by the illness in question. Such experience may make patients more vulnerable and make them proactive regarding taking actions to minimize the risk of disease. Studies in patients with type 2 diabetes have demonstrated the same showing that newly treated patients are less adherent than patients taking antidiabetic medications for a longer period of time. Newly diagnosed patients are usually symptom free and devoid of diabetic complications, thus having low perceived susceptibility to complications leading to decreased adherence. Also, if the disease does not affect a patient's functionality and quality of life, and diabetes is well controlled through lifestyle changes, then the patient may believe that medications are not needed. Newly diagnosed patients may also remain for prolonged periods in a stage of denial and refuse any interventions related to the control of diabetes. Additionally, newer patients might be less informed about their condition because providers fail to give adequate counseling until negative treatment outcomes and diabetic complications become evident. ${ }^{18}$

\section{$\underline{\text { Patient related attributes }}$}

Socioeconomic factors and patient demographics may play an important role in non-adherence. Indigent population without insurance may be non-adherent because of the cost of medications. Lower socioeconomic and minority groups generally have greater burdens of chronic disease and poorer outcomes. Members of these populations may also have barriers to adherence that thwart efforts at improving care and outcomes. ${ }^{94-}$ ${ }^{96}$ In addition to economic and access factors, these include lack of social support, differences in health beliefs and cultural norms, lower disease-specific knowledge and 
educational background, substance abuse and other physical or mental impairment, and care continuity and physician relationship issues.

Conflicting results have been found in terms of the association between age and adherence. A study by Schectman and associates ${ }^{89}$ in patients with diabetes found a $1.8 \%$ increase in mean adherence to oral hypoglycemics per decade of increased age. The association between age and adherence was consistent with that found by both Billups and colleagues ${ }^{97}$ and Bailey and associates ${ }^{98}$. However, studies by Monane and colleagues $^{99}$ and Gurwitz and associates ${ }^{100}$ conflict with respect to an association between age and adherence among senior citizens. The association of age and adherence could stem from training and/or maturing effects that facilitate adherence behavior balanced by increasing risk for decreased mental and physical functioning that may impair adherence with advanced age.

Race may also play an important role in affecting the adherence to medications. Schectman and associates ${ }^{89}$ reported that African-American diabetes patients averaged 4.1\% lower mean adherence than white patients. Medicare studies have shown similar racial disparities for intervention services after controlling for income. ${ }^{101}$ AfricanAmerican patients may have different perspectives and experiences with respect to health care that influences adherence behaviors. ${ }^{102}$ Others have shown that cultural and communication barriers, between African-American patients and Caucasian physicians can lead to less participatory decision making and greater mistrust, leading to lower adherence. ${ }^{103,104}$ Lower educational level has also been associated with mistrust of medical care and therefore potentially lower adherence. ${ }^{105}$ Because education status of African-American and white low-income patients may differ, education may confound 
this association between race and adherence. However, a recent survey found that minority ethnicity was a strong negative predictor of adherence among elderly patients without prescription coverage, independent of income and education. ${ }^{106}$

The Sick Role Theory suggests that non-adherent patients do not adopt the sick role, and fail to conduct behaviors appropriate to their illness. An important factor involved in the Sick Role Theory is denial, which is in turn associated with a negative effect on health outcomes, including regimen adherence. ${ }^{48}$ A significant relationship between self-perception of health and adherence with health care regimes has also been demonstrated. In a cross sectional convenience sample study of 106 subjects with a chronic illness for at least two years, Wichowski and Kubsch studied the relationship between self-perception of illness and adherence with health care regimens. There was a significant negative correlation between self-perception of illness and adherence for the total population. Adults who do not perceive themselves as ill are unlikely to comply with their treatment regimens. ${ }^{107}$

Although it is a durable belief that personality traits are predictive of patient adherence to treatment regimens, this has not been corroborated by the literature. However, depression has been consistently associated with poor adherence. Depression may contribute to low motivation, and it may impact directly on the will of the patients to get better, interfering in the patient's adherence to the treatment regimen. 


\section{Impact of Depression in Adherence and Outcomes in General}

Adherence to pharmacological regimens for depression is very poor, with less than half of those receiving an antidepressant prescription completing the recommended treatment. ${ }^{27}$ However adherence to medications for other conditions is also affected by depression. $^{39}$ Depression can affect cognitive focus, energy, and motivation thus a patient's willingness and ability to adhere to treatment recommendations including medication regimens. ${ }^{23}$ Positive expectations and beliefs in the benefits and efficacy of treatment have been shown to be essential to patient adherence. ${ }^{108}$ Depression often involves an appreciable degree of hopelessness and adherence might be difficult for a pessimistic patient. Considerable research has shown that presence of a social support network aids in improving adherence with regimens. ${ }^{109,110}$ Depression is often accompanied by social isolation and withdrawal from the very individuals who would be essential in providing emotional support and assistance. Also, depression might be associated with reductions in cognitive functioning essential to remembering and following through with recommendations. A meta-analysis of 12 published studies revealed that depressed patients were three times as likely as non depressed patients to be non adherent to recommendations given by physicians. ${ }^{23}$ Six of these studies involved patients with end stage renal disease or renal transplants and six involved other medical diseases (angina, cancer, arthritis). Patients with a recent Myocardial Infarction (MI) who dropped out of a recommended cardiac rehabilitation program were found to be more depressed and socially introverted than those who participated for the prescribed duration of therapy. Depression and poor motivation were also found to be inversely related to smoking cessation and increasing exercise activity for patients hospitalized for 
unstable angina or acute MI. ${ }^{111}$ In another study, elderly patients with depression who had coronary artery disease adhered less often to prescribed aspirin therapy than patients without depression. ${ }^{112}$

Recent studies conducted in primary care patients have found significantly higher health care costs in patients with depression as compared to patients without depression. This increase in costs was seen in primarily all categories such as primary care visits, laboratory test, emergency room visits, hospitalizations and pharmacy costs. ${ }^{113-115}$ Simon and associates demonstrated that depressed patients in an HMO had healthcare costs of approximately $\$ 4,246$ as compared to costs of $\$ 2,371$ in a comparison group. These numbers remained significantly different even after adjustment for presence of other chronic medical illnesses. ${ }^{113}$ Callahan and colleagues also found that patients with depression had mean total outpatient charges of $\$ 1,210$ over a nine-month period compared to $\$ 752$ for a non-depressed population after controlling for medical diagnoses. ${ }^{114}$ Similar results were shown by Unetzer and associates in an elderly cohort of 2,588 patients from a large HMO wherein the total median medical costs over a one year period for depressed patients was $\$ 2,147$ as compared to $\$ 1,461$ for non depressed patients. ${ }^{115}$ Primary care studies have also found that patients with major depression are disproportionately represented among high utilizers of medical care. In a study conducted in the Group Health Cooperative, it was found that $10 \%$ of patients use more outpatient visits, specialty medical visits, and in-hospital days than the $50 \%$ of lowest utilizers of these clinics. More than half of these high utilizers had significant depressive symptomatology. ${ }^{116}$ 


\section{Impact of Depression on Adherence and Outcomes in Type 2 Diabetes}

Studies have demonstrated that depression can play an important role in affecting adherence with prescribed regimen and thus directly or indirectly affect management and outcomes of a number of chronic conditions. ${ }^{23}$ Similar results are also seen in studies examining the effect of depression on outcomes related to type 2 diabetes. A metaanalysis was conducted to examine the association of depression with glycemic control in patients with diabetes. ${ }^{117}$ The diagnosis of depression was established by using semistructured clinical interviews and diagnostic criteria. Depression was quantified using self report instruments such as the Beck Depression Inventory (BDI) and Center for Epidemiological Studies-Depression Scale (CES-D) that measure the severity of recent depression symptoms, while glycemic control was assessed using a measure of A1C. The results of the meta-analysis indicated that depression was significantly associated with hyperglycemia. Although the results demonstrated that depression accounts for a small amount (3\%) of the variance in A1C levels, this is not trivial in clinical practice. Six studies examining the impact of depression on glycemic levels were restricted to patients with type 2 diabetes. ${ }^{118-123}$ Of these, two clearly showed a relationship between depression and glycemic control. ${ }^{118,119}$ Van der Does and associates ${ }^{118}$ found that higher A1C levels were significantly associated with symptom scores of worse mood on the Dutch Shortened Profile of Mood states and general well being in the Affect Balance Scale. Lustman and colleagues ${ }^{119}$ reported that depressed patients as indicated by the National Institute of Mental Health Diagnostic Interview Schedule Version Three, had higher A1C levels compared with those who were never depressed. Wilson and colleagues $^{120}$ found that depression as measured by BDI and CES-D was not significantly 
correlated with glycemic control but was significantly associated with self-care behaviors such as medication adherence, glucose testing, diet and exercise. The remaining three studies of depression and type 2 diabetes produced equivocal results. ${ }^{121-123}$ Peyrot and Rubin $^{121}$ found that although scores on the CES-D depression scale were higher in individuals with elevated A1C levels, the relationship was not statistically significant.

If depression is associated with hyperglycemia and hyperglycemia is associated with diabetes complications, then it follows that depression may also be associated with diabetic complications. Previous studies have correlated depression with a variety of diabetes complications such as diabetic neuropathy and cardiovascular disease, yet others have failed to find an association between depression and diabetic retinopathy and other diabetic complications. A recent meta-analysis of 27 studies was conducted to examine the impact of depression on diabetes complications. ${ }^{124}$ The results of this meta-analysis revealed a consistently statistically significant relationship between depression and a variety of diabetes complications. The overall effect size for the meta-analysis was statistically significant and in the small to moderate range depending on the specific complications. For each of these complications an increase in depressive symptoms was associated with an increase in the severity or number of diabetes complications.

These effects of depression on glycemic control and diabetic complications also translate into differences in health care costs. Data from the Medical Expenditure Panel Survey (MEPS) 1996 was used by Egede and associates ${ }^{125}$ to examine differences in health care expenditures in diabetes patients with and without depression. Expenditures were adjusted for inflation with the consumer price index to reflect 2001 dollars. Expenditures for prescription medication were higher in depressed individuals than in 
non-depressed individuals with diabetes $(\$ 1,392$ versus $\$ 666, \mathrm{p}<0.0001)$. There were no statistically significant differences in other expenditure categories. Co-morbid depression was associated with an almost fivefold significant increase in total health care expenditures. Significant differences were obtained even after controlling for age, sex, race, insurance status, and comorbidity. However, the values reported were unadjusted and the number of diabetics with depression was a very small proportion $(\mathrm{n}=85)$. This issue was also examined by Black ${ }^{126}$ in older diabetic Mexican Americans, using longitudinal data from the Hispanic Established Population for the Epidemiologic Study of the Elderly (EPSE). The risks of co-morbid myocardial infarction, hypertension, arthritis, and angina were significantly higher in the presence of concomitant depressive symptoms, as were the risks of diabetic complications, functional disability, incontinence, vision impairment, poorer perceived health status, and health care utilization among both diabetic and non-diabetic individuals. Rates were substantially higher among depressed individuals with diabetes, however, in comparison to depressed nondiabetic individuals. The study also demonstrated that the interaction of diabetes and depression was synergistic predicting greater mortality, greater incidence of both macro-and microvascular complications over the follow up period of seven years. The study also demonstrated a gradient response such that the risk of adverse events increases with the increasing severity of depression. Importantly, these health burdens were evident even when controlling for socio-demographic risk factors, including sex, age, education, marital status, immigration status, and living arrangements.

All these studies used self-reported measures for identification of depression and diabetes. Utilization and costs were also self-reported and were measured and analyzed 
in terms of total health care costs, which also included costs for mental health treatment. Regardless of whether major depression adversely affects an individual's physical health, total costs are expected to be higher for those with major depression due to the costs associated with mental health treatment. A recent study by Finkelstein and associates ${ }^{127}$ examined this issue using data from the 1997 Medicare 5\% Standard Analytic Files. This was the first study studying the impact of depression on non-mental health care costs in patients with diabetes using actual claims data to measure utilization. The study results indicated that controlling for age, gender, race, and comorbidities, non-mental health care costs were approximately $21.00 \%$ higher for depressed patients with diabetes as compared to non-depressed patients with diabetes. The authors also concluded that depressed patients seek treatment for more services and when admitted, spend more time in inpatient facilities than patients without depression.

The only study which examined the effect of depression on both adherence with oral hypoglycemics and health care utilization was conducted recently by Ciechanowski and associates ${ }^{28}$ in a primary care based sample of 367 patients with type 1 and type 2 diabetes in a staff model HMO. Depressive symptoms were assessed with the Hopkins Symptom Checklist-90 Revised. The patient population was divided into low, medium, and high depression tertile categories based on depression scores on the Hopkins symptom checklist. Adherence was assessed by self-report and by using an automated data collection system for refills of oral hypoglycemics. The authors found that depressive symptom severity was significantly associated with decreased adherence to dietary recommendations and approximately twice as many interruptions in refills of oral hypoglycemics. Depressive severity was associated with a non-significant increase in 
A1C levels. After controlling for demographics, medical comorbidity, and diabetes severity, higher levels of depression severity were associated with significantly worse mental and physical functioning and a significantly greater probability of having an emergency department, primary care, specialty care, medical inpatient, and mental health costs compared to patients with low severity depression. The total costs of the high tertile group were $\$ 3,654$ versus $\$ 2,653$ in the medium depression tertile and $\$ 2,094$ in the low tertile groups after adjusting for diabetes severity and medical co-morbidity.

However, this study was conducted in a small sample of diabetes patients in a highly specialized clinical setting. There was also the issue of non-response bias as only $62 \%$ of the originally contacted diabetes patients agreed to participate in the study. The study included a mix of type 1 and type 2 diabetes patients with varying durations of diabetes. As discussed in detail before, adherence issues might be drastically different in newly diagnosed patients as compared to a survivor cohort and hence need to be examined separately. Also, measures used to assess depressive diagnosis and a few other control variables were self-reported. Adherence to oral hypoglycemics was obtained through pharmaceutical claims data. However, refill data to measure adherence was only available for a subset of the population $(n=200)$. Most importantly, total health care utilization and costs were examined rather than specifically examining diabetes related outcomes and utilization.

Most of the studies in literature addressing this issue of depression and diabetes were cross sectional in nature thus not indicating whether depression causes aggravation of diabetes symptoms or problems with diabetes management lead to depression. This 
presence of endogeneity also can lead to statistical biases in the estimates of the impact of depression on diabetes related outcomes.

\section{Theories for Impact of Depression on Type 2 Diabetes Outcomes}

Speculation regarding the pathways by which depression impacts diabetes falls into two broad categories: psycho-behavioral and patho-physiological. Depression may influence diabetes through decreased motivation to maintain behaviors that will protect against the development or worsening of diabetes, such as proper weight, diet, medication adherence and exercise. ${ }^{23,28}$ Depression is associated with increased smoking, alcohol consumption, and unhealthy eating, and with diminished activity. ${ }^{44,45}$ People with diabetes who are depressed care for their diabetes less actively. Remission of depression may improve glycemic control by reducing unhealthy behaviors and promoting healthy behaviors. Diabetes patients who are depressed are more likely to report limited physical functioning and increasing physical activity may be one of the most important behavioral changes for diabetes patients. Epidemiologic studies have repeatedly found cross sectional association between low levels of physical activity and depression. Studies indicate that patients who are more physically active have better depression symptomatology, and that individuals who are less physically active are more likely to develop depression. ${ }^{128-130}$

Alternatively, metabolic alterations associated with depression may disrupt normal glucose metabolism. The increase risk of negative diabetic outcomes may be the result of biological changes such as neurohormonal and neurotransmitter changes. Studies suggest that depressive disorders are accompanied by increased sympathoadrenal system and hypothalomapituitary adrenal axis activity causing increased release of 
counter regulatory hormones and cortisol, respectively, which increase insulin resistance and decrease glucose uptake. Lower immune functioning or inhibited cortisol release may also increase insulin resistance and thus increase vulnerability to diabetic complications. $^{42,43}$

It may also be possible that both depression and diabetes share the common pathogenesis such as actions of the autonomic or sympathetic nervous systems, or polymorphism of genes associated with obesity. Thus, the link between depression and diabetes may be the direct or indirect result of risk factors common to both conditions, such as obesity, inactivity, medication use, and other preexisting psychological and physical conditions.

In view of the alternate theories for the impact of depression on diabetes, an important question that needs to be addressed is whether poor adherence actually mediates the relationship between depression and outcome. One approach to this question is to determine whether the association between depression and type 2 diabetes outcomes is weakened after adjusting for adherence. Gary and associates ${ }^{131}$ found that presence of depression was associated with higher cholesterol, triglycerides, and glucose levels in patients with type 2 diabetes. However, adjusting for adherence to diet, physical activity, smoking, glucose monitoring, and medications did not reduce the effect of depression. Another approach to determining the role of adherence as a mediator is to examine whether interventions that reduce depression and improve type 2 diabetes outcomes also show improvements in adherence. Lustman and associates ${ }^{122,123}$ conducted two such intervention studies for depression in patients with diabetes. In both studies, the intervention reduced depression and improved glucose control relative to the 
control condition. However, there was no evidence that reducing depression led to improved adherence, as measured by the use of home blood glucose monitoring. However, the Cognitive Behavioral Therapy (CBT) routinely included homework assignments directing patients to record their thoughts and increase various physical and social activities. Thus, it is possible that the participation in CBT complicated an already complex regimen and thus decreased adherence with self-monitoring of blood glucose levels.

Thus, based on these few studies there is lack of evidence to support the role of adherence as a mediator in the relationship between depression and diabetes outcomes. Further research with objective measures of adherence, clear criteria for defining adherence and depression, and large samples with longitudinal follow-up need to be conducted.

\section{Epidemiology of Depression and Type 2 Diabetes}

These effects of depression on diabetes related outcomes could be highly detrimental, especially if the rate of occurrence of depression in patients with type 2 diabetes is high. Hence, the epidemiological burden of depression in patients with type 2 diabetes needs to be evaluated and the existing literature on this issue will be discussed in the following sections.

\section{Prevalence of Depression in Patients with Type 2 Diabetes}

A number of studies examining the prevalence of depression in patients with diabetes have been published in recent years. These studies have used various patient population, research designs, and diagnostic tools. The varying prevalence rates may reflect the differences in assessment methods (self-reported depression scales or 
structured diagnostic interviews), sample differences (community based or clinical samples), and type of diabetes (type 1 or type 2). Also, a few of these studies were conducted without the presence of a control group. As control groups are essential and basic to epidemiological research, the following review will focus exclusively on controlled studies performed in patients with type 2 diabetes.

A recent meta-analysis of 42 studies indicated that the odds of depression in patients with diabetes were twice that of a comparison group of patients without diabetes. ${ }^{29} \mathrm{~A}$ sub-analysis on the basis of seven controlled studies including only patients with type 2 diabetes indicated that patients with type 2 diabetes were nearly two and a half times more likely to have co-morbid depression as compared to patients without type 2 diabetes. ${ }^{32,132-137}$ Only two of the seven controlled studies used structured diagnostic interviews for the assessment of depression. ${ }^{32,137}$ The structured diagnostic interviews used were the National Institute of Health's Diagnostic Interview Schedule (DIS) and the Clinical Interview Schedule (CIS). The DIS is an instrument for making psychiatric diagnoses in accordance with criteria specified in the DSM-III of the American Psychiatric Association, while the CIS reports psychiatric diagnoses according the International Classification of Disease criteria. Both the interviews have been found to be reliable and valid in assessing depressive disorders in patients with type 2 diabetes. The remaining controlled studies used self-reported depression scales such as the Geriatric Depression Scale (GDS), Zung scale, and the Beck Depression Inventory (BDI). ${ }^{132-136}$ In these studies, depression prevalence was equal to the percentage of subjects with scale scores above a specified threshold value. However, this threshold value varied across the studies. The method of patient enrollment also differed between the studies. Five 
controlled studies $^{32,132,133,135,137}$ identified the type 2 diabetes patients and their controls randomly from a community population, while the remaining two studies ${ }^{134,136}$ enrolled the patients and their controls without diabetes from health care clinics, patient support groups, or physician referrals. The odds of depression were significantly elevated in patients with type 2 diabetes as compared to control groups irrespective of the method of depression assessment or patient enrollment. However, only of a few of these studies explored the effect of concomitant medical illnesses on the association between depression and type 2 diabetes. This is important as medical conditions in general are a risk factor for depressive disorders and the presence of type 2 diabetes may not add any additional risk. Thus, it is possible that the concomitant medical illnesses accompanied with type 2 diabetes may contribute to this observed increase in prevalence of depressive diagnosis.

Weyerer and associates ${ }^{137}$ observed that although patients with type 2 diabetes had a higher prevalence of depressive symptoms than those with no somatic illness, no differences were found when diabetes patients were compared to patients with other somatic illnesses. A recently published study by Pouwer and associates ${ }^{138}$ displayed similar results. This community-based study was conducted in elderly Dutch adults. Depression was assessed using the CES-D scale and diagnosis of type 2 diabetes was obtained from self-reports and data from general practitioners. The results of this study indicated that the $20 \%$ of the patients with type 2 and other co-morbid chronic conditions suffered from pervasive depression. Rates of depression were found to be considerably lower in patients with type 2 diabetes only $(8 \%)$ and healthy control subjects $(9 \%)$. Thus, compared to healthy subjects, the odds for depression are particularly increased in 
patients with type 2 diabetes and other co-morbid diseases, but not in those with type 2 diabetes alone. However, all the studies mentioned above were not population based and had relatively small sample sizes leading to doubts regarding the stability of the prevalence estimates.

The only study using claims data to determine prevalence of co-morbid depression in patients with type 2 diabetes was conducted among enrollees of Kaiser Permanente Northwest (KPNW). ${ }^{139}$ The study found unadjusted depression prevalence rates of nearly $18 \%$ in patients with type 2 diabetes. After controlling for confounders such as age, gender, presence of cardiovascular disease, and weight the results indicated that patients with type 2 diabetes were slightly more likely to have co-morbid depression as compared to patients without diabetes $(\mathrm{OR}=1.187)$. However, the study failed to control for co-morbidities other than cardiovascular conditions. Hence there is a possibility of detection bias as individuals with type 2 diabetes are more likely to have higher number of co-morbid conditions and thus more likely to seek medical care than individuals without type 2 diabetes. Thus, individuals with type 2 diabetes would have a greater opportunity for being diagnosed with depression than those without type 2 diabetes. Also, the study did not examine the interaction effects between demographics and type 2 diabetes on the prevalence of co-morbid depression. The impact of age on the relationship between type 2 diabetes and depression is important as the peak period of onset for depression is generally in the early adult years between 20 and 30 years of age; while the onset of type 2 diabetes typically occurs after the age of 50 . Hence, in addition to adjusting for age it becomes important to control for a statistical interaction between age and presence of type 2 diabetes to provide unbiased estimates. 


\section{Incidence of Type 2 Diabetes in Patients with Depression}

The studies mentioned in the above section pertained to the concept of prevalence of depression and were all cross sectional in nature. In addition to prevalence of comorbid depression in patients with type 2 diabetes, it is also important to consider the epidemiological measure of incidence. In 1684, Thomas Willis, a British physician

suggested that diabetes results from presence of pre-existing depressive disorders. ${ }^{140}$ Evidence from recent prospective studies have confirmed William's hypothesis and indicated that depression doubles the risk of incident type 2 diabetes even after controlling for other risk factors. This finding is of important significance as recent research demonstrates the possibility of preventing or delaying the progression from impaired glucose tolerance to type 2 diabetes through moderate exercise, weight loss, modification of diet, or intake of metformin. Depressive disorders can affect the adherence to each of the above preventive activities and thus lead to increased onset of type 2 diabetes.

Eaton and associates ${ }^{32}$ conducted the first prospective study examining the effect of depression on incidence of type 2 diabetes in adult household residents participating in the Epidemiological Catchment Area (ECA) Program Survey in Baltimore, Maryland. Results of the study indicated that controlling for age, race, sex, socio-economic status, education, use of health services, and body weight, community respondents with major depression diagnosed by using the National Institute of Mental Health's Diagnostic Interview Schedule had an estimated relative risk of 2.23 of developing adult onset of diabetes mellitus over a 13-year period. However, the diagnosis of diabetes was by selfreport and the study failed to control for all the important risk factors for incidence of 
diabetes. Self-reported diagnosis of diabetes could have also led to the problem of undetected diabetes. It is possible that some people enrolled in the cohort may have had onset of type 2 diabetes at baseline, thus biasing the study results.

Kawakami and associates ${ }^{33}$ conducted a methodologically superior study in a sample of 2,764 male employees of an electrical company in Japan. The study results indicated that significant depressive symptoms measured according to the Zung SelfRating Depression scale were associated with a 2.3 times increased risk of developing type 2 diabetes compared to non depressed employees after controlling for known risk factors over a eight year follow up period. The results of this Japanese study are presumably more valid as the study used diagnostic measures such as urine tests and oral glucose tolerance testing for identification of type 2 diabetes. The study also controlled for important diabetes risk factors such as body mass index, smoking, alcohol consumption, activity level, and family history of diabetes. Biases due to presence of undetected diabetes at baseline were also ruled out by consistent results a sub-analysis excluding cases found in the first half of the follow up period. However, both these studies had small number of incident cases ( $\mathrm{n}=89$ and 43 respectively) and thus had limited power. The study populations were also not representative of the general US population.

A couple of studies have recently used data from the National Health and Nutrition Examination Epidemiologic Follow-up survey (NHEFS) to test the relationship between depression and incidence of diabetes. This data is nationally representative and provides a large cohort of participants for follow up over an extended period of time. One of the studies used the NHEFS cohort ${ }^{34}$ from the second examination (1982-1984) 
and measured depression using the CES-D. During the follow up period of approximately nine years, there were 465 incident cases of diabetes. After controlling for demographic characteristics, the relative hazard $(\mathrm{RH})$ of incident diabetes among those with high depressive symptoms was 1.27 as compared to those without symptoms. Additional statistical controlling for known diabetes risk factors such as body mass index and physical activity further decreased the strength of the association $(\mathrm{RH}=1.11)$. Both these values were statistically insignificant at $\mathrm{p}<0.05$ and thus the study failed to find any significant effect of depressive symptoms on diabetes incidence. However, a subsequent study conducted in the NHEFS cohort ${ }^{35}$ from the first examination (1971-1975) measuring depressive symptoms using the General Well-Being Depression subscale suggested a significant and independent role of depressive symptoms in the development of diabetes. Over an average of 15.6 years of follow-up, 369 participants developed diabetes. The incidence of diabetes was highest among participants reporting high numbers of depressive symptoms ( 7.3 per 1,000 person-years) and did not differ between persons reporting intermediate and low numbers of symptoms (3.4 and 3.6 per 1,000 person-years, respectively). The association between depressive symptoms and incidence of diabetes was especially stronger in participants with low education levels. These results also persisted in multivariate models after adjusting for demographics (age, gender, and race), health behaviors (smoking, alcohol use, and physical activity), and baseline body mass index.

Most of these studies relied on self-reported data for identification of diabetes and depression. Each study exploring the relationship has used a different scale to measure the self-report of depressive symptoms and each scale measures depressive symptoms 
over a different time interval. Also, the studies using data from the NHEFS cohort were not specific to incidence of type 2 diabetes and failed to adequately address the issues of undetected diabetes at baseline. Additionally, studies reported in the literature assessed depressive symptoms only once at baseline and hence during the follow-up period a nondepressed subject could have developed depressive symptoms and subsequent type 2 diabetes resulting in misclassification bias. This bias could be significant especially due to the long follow-up periods in these studies ranging from eight to 16 years.

\section{Overview of the Medicaid Program}

As the study will be conducted using claims data for enrollees of West Virginia Medicaid, it will be helpful to understand the structure and functioning of this health care system. Medicaid is a federally sponsored health insurance program for low-income, disabled and members of families with dependent children initiated in 1965. The federal government provides the fiscal assistance and the basic framework of regulations, guidelines, and operation policies. ${ }^{141}$ However, the state governments are typically responsible for administration of the Medicaid program. Benefits provided by Medicaid include coverage for physician visits, inpatient and outpatient hospitalizations, laboratory testing, nursing home care, family planning services and supplies, and home health care. The federal government mandates these health care benefits. Additionally, there are optional services that are left to the discretion of each state. Although optional, all states provide reimbursement for prescription costs.

In recent years there has been widespread concern regarding the tremendous growth in health spending through Medicaid. Total federal and state Medicaid expenditures including administrative costs increased form $\$ 58$ billion in 1989 to $\$ 194.7$ 
billion in 2000. One of the major reasons for this increase in expenditures is the increase in size of the Medicaid eligible population. In 1990, there were 25.3 million enrollees, which increased to 36.3 million in 1995 and more than 44 million in $2000 .{ }^{142,143}$ A similar trend is also seen in West Virginia Medicaid with total number of recipients in the program increasing from 178,254 in 1982 to 354,326 in 2000 . This growth in number of recipients has resulted in a dramatic increase in expenditures from \$121 million in 1982 to over 1.391 billion in $2000 .{ }^{143}$ This can also be interpreted as an increase in average spending per recipient from $\$ 678.84$ to approximately $\$ 3,900$. Thus, it becomes important to identify areas of cost-containment; effect of depression on other chronic conditions such as type 2 diabetes being one such area.

West Virginia's Medicaid program consists of three components: ${ }^{144}$

- Physician Assured Access System (PAAS); a primary care case management program wherein individuals enrolled in PAAS receive case management and education services not available to individuals enrolled in the fee-for-service program. Under the program, participants select a primary care provider who coordinates care and whose approval is required for most health care services.

- Mountain Health Trust (MHT); a capitated program similar to being enrolled in a Health Maintenance organization (HMO).

- Traditional fee-for-service program

In 2002, 281,526 individuals were enrolled in West Virginia's Medicaid program. As of June 2002, 97,174 Medicaid recipients were enrolled in PAAS. In 1996, Mountain Health Trust (MHT) was developed under a subsequent 1915(b) waiver that allowed the state to require a majority of its Medicaid recipients including individuals with 
disabilities to enroll within an HMO. As of June 2002, 48,189 Medicaid recipients who qualify through Temporary Assistance for Needy Families (TANF) were enrolled in MHT, and Medicaid recipients who qualified through the supplementary security income (SSI) were eligible for the primary care case management program and not the capitated program. ${ }^{145}$ 


\section{CHAPTER THREE}

\section{METHODOLOGY}

\section{Data Source}

\section{$\underline{\text { West Virginia Medicaid claims data }}$}

State Medicaid programs provide health care coverage to poor, aged, and disabled individuals. The Bureau for Medical Services of the West Virginia Department of Health and Human Resources has been responsible for the management of the West Virginia Medicaid Program (WVMP). The West Virginia Medicaid Bureau contracts with Consultec, Inc. (Atlanta, Georgia), to serve as its claims processor. Consultec maintains and operates the Medicaid Management Information Systems (MMIS), which processes provider claims and payments. MMIS data comprises of three files - provider files, recipient files, and claims (medical and pharmacy) files. The following is a description of these files and the specific fields (variables) contained in each file.

The provider file contains specific information about all health care providers eligible to deliver services to Medicaid recipients. It primarily contains the provider's name, specialty, Medicaid eligibility, and tax related information. The recipient file contains detailed information about Medicaid recipients. Important fields in this file include recipient's Medicaid number, period of Medicaid eligibility, period of managed care eligibility, unique identification number, date of birth, gender, race, and county of residence. The claims file stores detailed information specific to processed claims. For each medical claim, the file contains fields such as invoice type, provider number, recipient number, International Classification of Disease $9^{\text {th }}$ edition (ICD-9) ${ }^{146}$ code of diagnosis for which the service was provided, Common Procedural Terminology (CPT) 
code for procedures and services provided, Diagnostic Related Group (DRG) codes, date claim was submitted, date of adjudication, through-date of service, coordination of benefit code, primary carrier code, extract indicator, and total amount paid. The medical claims are further classified into two files: outpatient claims and emergency room/hospitalization claims. For pharmacy claims, the file contains fields such as number of days supply, metric quantity, National Drug Classification (NDC) code, generic code, therapeutic class code, refill number, provider ID number, and amount paid.

\section{Study Population}

West Virginia Medicaid data was extracted for the period 01/01/1997 to $12 / 31 / 2002$. Thus, final data used for the study included all paid claims data for all individuals eligible for West Virginia Medicaid during the study period. Since Medicaid recipients aged 65 years and older are eligible for coverage under both Medicaid and Medicare, the subjects of this research were limited to all Medicaid recipients who were younger than 65 years to avoid the issue of coverage under both Medicaid and Medicare. For similar reasons, Medicaid recipients who were part of managed care were not included in the study. 


\section{Data Extraction}

The West Virginia Medicaid claims data obtained from Consultec is loaded on the server maintained by West Virginia University’s Rational Drug Therapy Program (RDTP). The data needed for this study was extracted through the software BrioIntelligence and converted into Statistical Package for Social Sciences (SPSS) data sets. The extraction process involved identifying the study subjects using specific selection criteria and the fields/variables needed were downloaded for the defined study time period.

\section{Data Cleaning}

As mentioned previously, the medical claims files are aggregated into two separate files of outpatient claims and ER/hospitalization claims. It is preferable to separate the ER claims from the hospitalization claims to get a better distribution of these health care costs. A variable termed as hospitalization Extract Indicator (EXTIND) in the ER/hospitalization claims classifies the claims as those belonging to an ER visit or a hospitalization episode. This variable along with the information provided by the Diagnosis Related Group (DRG) codes and CPT codes was used to separate ER and hospitalization claims. The following algorithm was used to separate claims into "ER" and "Hospitalization" files:

- Hospitalization claims from the ER/hospitalization file were identified on the basis of a DRG code greater than 0 . These claims were separated and classified into the "Hospitalization" file. 
- ER claims were identified on the basis of the EXTIND code. All claims with EXTIND $=2$ are indicative of an ER visit. These claims along with all claims with the same date of service were separated and classified into the "ER" file

- The outpatient files contain a few claims, which are related to ER episodes. These files were identified on the basis of CPT codes ranging from 99281 to 99285. These claims along with all claims with the same date of service were separated and transferred to the "ER" file.

- If the ER events lead to hospitalization, the ER event was considered as a component of the subsequent hospitalization episode to prevent double counting. Claims from the "ER" file with the same date as a hospitalization claim were identified as indicative of ER episodes subsequently leading to hospitalization and were transferred to the "Hospitalization" file.

- A large proportion of claims in the ER/ hospitalization files included services, which are outpatient but performed in a hospital setting and hence needed to be aggregated as outpatient claims. After the algorithm mentioned above was used to classify claims into ER or hospitalization claims, the remaining claims in the ER/hospitalization files were classified as outpatient claims and were transferred to the outpatient files.

In addition, there was also the issue of duplication of claims in the medical and pharmacy files that had to be addressed. These duplications were primarily due to initial rejection of claims. If a claim was rejected, it was followed up with a negative claim. Both these negative and positive claims were deleted from the database. 


\section{Selection Criteria}

Type 2 diabetes

ICD-9 CM diagnosis codes from medical claims were used to identify patients with type 2 diabetes. The type 2 diabetic patients were identified on basis of ICD-9 CM diagnoses codes from $250.0 \mathrm{x}$ through $250.9 \mathrm{x}$, where $\mathrm{x}$ is the fifth digit with a value of 0 or 2. Individuals having diagnosis codes with a missing fifth digit cannot be classified as type 1 or type 2 diabetes patients and a decision was made on their inclusion depending on their use of oral hypoglycemics. The following criteria for classification was used: at least one inpatient admission for which the principal diagnosis was recorded as type 2 diabetes, or at least two inpatient admissions or two outpatient facility or physician office visit claims for which any diagnoses was recorded as type 2 diabetes. Claims for laboratory, pathology or radiology services were not used to identify individuals with diabetes, since their use could incorrectly identify individuals as having diabetes based on diagnostic procedures such as screening.

\section{$\underline{\text { Depression }}$}

The depressive diagnoses considered in this study included single episode major depressive disorder (ICD-9 CM diagnoses codes 296.20 through 296.26), recurrent episode major depressive disorder (ICD-9 CM diagnoses code 296.3), neurotic depression/chronic depression/dysthymia (ICD-9 CM 300.4), and depression not otherwise specified (ICD-9 CM 311). As mental disorders such as depression are associated with issues of initial misdiagnosis, at least one inpatient admission for which the principal diagnosis was recorded as depression, or at least two inpatient admissions or two outpatient facility or physician office visit claims for which any diagnoses was 
recorded as depression was needed. Patients with inadequate number of depressive diagnoses were excluded from the analysis to prevent any misclassification bias.

The methods for the 2 phases of the study are described in more detail below:

\section{Phase 1}

\section{Objective 1}

\section{Prevalence of co-morbid depression in patients with type 2 diabetes}

Number of type 2 diabetic patients with

Prevalence rate $=\quad$ co-morbid depression during the year $2000 \times 100,000$

per $100,000 \quad$ Number of patients with type 2 diabetes

during the year 2000

Patients in West Virginia Medicaid with a diagnosis of type 2 diabetes in year

2000 were identified based on the specified selection criteria. Among these patients with type 2 diabetes, presence of co-morbid depression was identified based on the selection criteria for depression.

In addition, a group of Medicaid enrollees without type 2 diabetes diagnosis or prescription of diabetic agents was identified in year 2000. Presence of co-morbid depression was also determined for this group of patients. In addition to uni-variate chisquare analyses, multivariate logistic regression analyses were conducted to control for effect of confounders such as age, gender, race, and co-morbidity. Interaction effects of the main independent variables with age and gender were also included in the model as the associations between type 2 diabetes and depression may differ based on age and gender. As interaction terms were used in the model, the independent variable "age" was centered about the average age in the population for ease of interpretation. To separate 
the effects of type 2 diabetes and the presence of related co-morbidities on the prevalence of depression, a block-wise logistic model was created consisting of the following blocks of co-variates entered sequentially in the model.

Block 1: Demographic variables: age, gender, race, interaction term of age and diabetes, interaction term of gender and diabetes

Block 2: Presence of chronic co-morbidities unrelated to type 2 diabetes: Cancer, asthma, and chronic liver conditions.

Block 3: Number of physician office visits and presence of chronic co-morbidities related to type 2 diabetes (Cardiovascular conditions, cerebro-vascular conditions, nephropathy, retinopathy, neuropathy and endocrine/metabolic disorders).

The analysis was restricted to patients who were continuously Medicaid eligible and had no Medicaid HMO eligibility in the year 2000. Only patients between the ages of 18 and 64 were included in the analysis to exclude children and enrollees who were also eligible for Medicare.

\section{Objective 2}

\section{Incidence of type 2 diabetes in patients with pre-existing depression}

$\begin{aligned} & \text { Incidence rate }= \\ & \text { per 100,000 }\end{aligned} \begin{aligned} & \begin{array}{l}\text { Number of new cases of type 2 diabetes in patients with } \\ \text { preexisting depression occurring between 1998-2000 }\end{array} \\ & \text { Number of patients with depression between 1997 }\end{aligned} \times 100,000$

Patients with depression were identified on the basis of the inclusion criteria for depression in the year 1997. As a comparison group, a group of Medicaid enrollees without depression diagnosis or prescription of antidepressants was identified in the year 
1997. Patients with a medical claim for type 2 diabetes or a prescription for a hypoglycemic agent in the year 1997 were excluded from the analysis. These patients were then followed till the December 31, 2002 to identify incident cases of type 2 diabetes in both the groups. In addition to univariate chi-square analyses, multivariate logistic regression analyses were conducted to control for effect of confounders such as age, gender, race, and co-morbidity.

The analysis was restricted to patients who were continuously Medicaid eligible and had no Medicaid HMO eligibility in the period 1997-2002. Only patients between the ages of 18 and 60 were included in the analysis to exclude children and enrollees who were also eligible for Medicare.

\section{Phase 2}

To examine the impact of preexisting depression on patterns of use of oral hypoglycemics, drug adherence, and outcomes in patients with type 2 diabetes, the following methodology was employed:

Incident cases of type 2 diabetes were identified using the selection criteria mentioned before. The first prescription for an oral hypoglycemic agent was treated as an index prescription. A 12-month pre period without a diagnosis for type 2 diabetes or a prescription for an oral hypoglycemic was used to confirm that the patient is newly diagnosed with type 2 diabetes. A 12 -month pre period was also used to identify the diagnosis of depressive symptoms in these patients. Drug utilization patterns and adherence was measured over the period of 12 months post index prescription. Incident 
type 2 diabetes patients who do not have preexisting depression but develop depression in the follow up period were excluded from the analysis.

\section{Exclusion Criteria for Phase 2}

- Troglitazone belongs to the thiazolidenedione class of oral hypoglycemics. It was shown to have liver toxicity and hence required regular monitoring. Due to its toxic side effects the drug was finally removed from the market in the year $2000 .^{70}$ Thus, while observing the patterns of use and adherence in the database, one might attribute any switch from troglitazone as failure of therapy or lack of adherence. This can lead to misinterpretation of the results and hence patients who had any prescription for troglitazone in the study period were excluded from the analysis.

- Patients receiving antidepressant therapy without any depressive diagnoses were excluded from the analysis to prevent any misclassification bias.

- Patients with brief depressive reaction (ICD-9 CM 309.0), prolonged depressive reaction (ICD-9 CM 309.1), psychotic depression (ICD-9 CM 298.0) were excluded because these are etiologically different from major depressive episodes or chronic depression.

- In addition, patients with a diagnosis of schizophrenia (ICD-9 CM 295.10295.30,295.60, 295.90), bipolar disorder (ICD-9 CM 296.0, 296.1, 296.4, 296.6), dementia (ICD-9 CM 294.1, 294.9), conditions involving cerebral degeneration such as Alzheimer's disease (330.XX, 331.XX), neurotic disorders such as anxiety, hysteria, phobic disorders, obsessive compulsive disorders and others (ICD-9 CM 300.XX except 300.4X) were also excluded from the analysis to 
avoid confounding of the effect of other mental disorders on adherence to oral hypoglycemics and related outcomes. These stringent criteria will help minimize misclassification bias for majority of the objectives of the study.

- Patients in long-term care facility, intermediate care facility or skilled nursing facility are a select population who are more severe and may demonstrate a high amount of adherence as they are under constant supervision. Hence, patients in such facilities in the follow up period were also excluded from the analysis.

- Patients who are not continuously eligible in the follow up periods for specific objectives were excluded from the study.

\section{Measurement of Variables}

Main independent variable:

\section{Co-morbid depression}

Co-morbid depression will be a categorical variable classified as follows:

- No diagnosis of depression

- Diagnosis of depression

A multivariate framework was used to estimate the impact of depression on adherence and other type 2 diabetes outcomes. All the multivariate models controlled for confounding factors such as demographics, co-morbidity, diabetes severity, complexity of regimen, and interaction with health care providers. Multiple variables were used to measure each of these confounding factors and are described in detail below: 


\section{Demographics}

Age: Age was obtained by calculating the difference between the recipient's date of birth and the index date of the study.

Gender: The field "recipient gender" in the Medicaid recipient data was utilized to identify patient's gender.

Race: The field "recipient race" in the Medicaid recipient data was utilized to identify patient's race. Patient's race was classified into 2 categories: whites and non-whites Geographical location: Patient's geographical location was categorized on basis of their county of residence. The 2003 Rural-urban Continuum Codes were used to classify the county of residence as rural or urban.

These codes form a classification scheme that categorizes counties by size and degree of urbanization and proximity to urban areas. Counties all across the nation have been subdivided into three metro and six non-metro categories, resulting in a nine-part county codification. This scheme was originally developed in 1974. This scheme of coding allows researchers to break county data into finer residential groups as per convenience. The Census Bureau made a radical shift in determining rural-urban boundaries by changing and liberalizing the procedures for delineating urbanized areas of 50,000 or more people, and abandoning place boundaries in measuring urban or rural population. The procedures used in defining Urbanized Areas were extended down to clusters of 2,500 or more people, based solely on population density per square mile. ${ }^{147}$ Year of index date: As our enrollment period spanned a period of three years (i.e. 19982000) a control variable for the year of index date was included to account for differences in treatment and diagnosis practices over time. This variable also accounts for 
introduction of oral hypoglycemics and marketing strategies that vary and thus affect prescribing behavior.

\section{$\underline{\text { Co-morbidity }}$}

Multiple variables were used to measure baseline co-morbidity for newly diagnosed type 2 diabetes patients. The Charlson co-morbidity index ${ }^{148}$ based on medical claims in the 12-month period prior to the index date was used to measure co-morbidity. In addition to the Charlson co-morbidity index, co-morbidity was measured using a mix of variables identifying the presence of specific co-morbid conditions and those related to utilization of health care services in the 12-month pre-period before the index date of an oral hypoglycemic agent.

Charlson co-morbidity index: The Charlson Index is a list of 19 medical conditions. Each condition has a weight assigned from one to six, which is derived from relative risk estimates of proportional hazard regression models using clinical data. The Charlson index is the sum of weights for all prevalent conditions during a specified time period. The index has been shown to have a strong monotonic association of approximately a two fold increase in mortality per increment in index level. There are a variety of indices that are primarily based on the Charlson comorbidity index. The version by D'Hoore and associates, ${ }^{149}$ which is one of the few Charlson indices for use with administrative claims data, was used in our study. As coding of the ICD-9 codes in administrative databases after the decimal places is not reliable, the Charlson index by D'Hoore and associates is based on only the first three digits of ICD-9 codes. Diagnosis codes related to depression and diabetes were excluded in the computation of this index. 
Utilization related variables: These variables measured overall utilization in the 12-

month pre-period excluding utilization accrued due to depression or antidepressants. The following utilization variables were used:

- Number of ER/hospitalization visits

- Total health care costs

- Distinct number of therapeutic medication classes for management of chronic conditions.

Specific co-morbid conditions: The presence of the following co-morbid conditions was identified in the 12-month pre period on the basis of their prevalence in the study population and their impact on outcomes related to diabetes management.

- Presence of cardio-vascular conditions (including hypertension and hyperlipidemia)

- Presence of Asthma

- Presence of Ulcers

- Presence of Cancer

\section{Complexity of drug regimen}

The following variables will be used in the model to control for the confounding effect of complexity of drug regimen on diabetes related outcomes:

Mean daily number of pills of oral hypoglycemic agents: This computation was based on the first oral hypoglycemic prescription. The fields "Qty dispensed" and "days supply" were used to compute number of pills per day. 
Number of prescriptions for chronic medications: All the available therapeutic classes of medication were categorized as those used for acute or chronic conditions on basis of clinical literature. Therapeutic classes such as Non-Steroidal Inflammatory Drugs (NSAIDs), which can be used for either chronic or acute purposes, were conservatively classified as those for acute conditions. Number of prescriptions for chronic conditions other than depression in the pre period was computed.

Mean daily number of pills of other chronic medications: Mean number of pills per day was computed for chronic medications other than those used for diabetes or depression. This analysis was restricted to prescriptions for orally ingested tablets or capsules in the 60-day period before the index date. This was computed by multiplying the mean number of pills per day for each prescription of a chronic medication with the number of prescriptions with unique generic codes in the 60-day pre period.

\section{Interaction with healthcare providers}

Physician Assured Access System (PAAS) enrollment: Individuals enrolled in PAAS receive case management and education services not available to individuals enrolled in the fee-for-service program. Under the program, participants select a primary care provider who coordinates care and whose approval is required for most health care services. Hence, a dummy variable indicating whether the patient is enrolled in PAAS or is under the traditional fee for service system was used as a proxy for interaction with the health care provider.

Pharmacy patronage: In addition to interaction with their physician, patient's interaction with their pharmacist can also affect adherence to medications and related outcomes. 
Pharmacy patronage measured in terms of number of pharmacies visited in the six-month pre period was used as a proxy for interaction with pharmacists. The variable was categorized as:

- No pharmacy use

- Single pharmacy use

- Multiple pharmacy use

\section{$\underline{\text { Severity of diabetes }}$}

Although all patients were newly diagnosed with type 2 diabetes, there might be differences in diabetes severity, which was controlled using the following variables: Initiation on monotherapy/polytherapy: A medication regimen was referred to as monotherapy when patients received therapy with a single class of oral hypoglycemic medication, and polytherapy when patients simultaneously received two or more classes of oral hypoglycemics. A pattern of use was classified as initial poly-therapy if one of the following criteria was satisfied:

- Index prescription was for a fixed combination therapy and the patients received at least two more prescriptions for the combination therapy in the follow up period; or

- The patient had a prescription claim for a second oral hypoglycemic agent/insulin (different class) within 15 days of the index fill date of the index prescription and received at least two more prescriptions for each of the drugs in the follow up period. 
Class of the index oral hypoglycemic agent: Class of the index oral hypoglycemic prescription can indicate the severity of diabetes and also predict diabetes outcomes through its efficacy and side effect profile. The index oral hypoglycemic prescription was classified into one of the following class of oral hypoglycemic:

First generation/second generation sulfonylurea, biguanide, thiazolidenedione, meglitinide, or alpha glucosidase inhibitor.

Specialty of the prescribing physician: Specialty of the physician who prescribes the index oral hypoglycemic prescription can be predictive of diabetes severity, access issues, and/or diabetes related outcomes. This variable was categorized as index prescription provided by: a specialist (endocrinologist), an internist, or a family physician.

Gap between diagnosis and medication therapy: Type 2 diabetes patients in our study may have varying lengths of time between initial diagnosis of type 2 diabetes and initiation of oral hypoglycemic therapy. This gap between diagnosis and initiation of medication therapy was controlled in our study as a continuous variable measured in days.

Diabetes related utilization: Although the study population consisted of only patients newly diagnosed with type 2 diabetes, the index date of the study period was the date of the initial prescription of oral hypoglycemic. Hence, diabetes related utilization was available for patients who had a gap in therapy between initiation of pharmacotherapy and initial diagnosis of type 2 diabetes and served as a good proxy for diabetes severity. The following variables were used to measure diabetes related utilization in the 12-month pre period: 
- Total type 2 diabetes related costs

- Number of type 2 diabetes related ER/hospitalizations

The variables described above were used as independent control variables in all the multivariate models to examine the impact of depression on type 2 diabetes outcomes. A variety of outcomes such as adherence with oral hypoglycemics, and utilization related to type 2 diabetes management were evaluated. The measurement of the outcomes is described in detail below:

\section{Patterns of oral hypoglycemic use}

Patterns of oral hypoglycemic use could be classified in any of the following categories based on utilization of prescriptions in the follow-up period.

\section{- Discontinuation}

A patient was deemed as a discontinuer in the 12-month follow up period after initiation of oral hypoglycemic therapy if he/she ends therapy on an oral hypoglycemic/insulin and does not receive a refill within $\mathrm{x}$ days of supply exhaustion where $\mathrm{x}$ equals $250 \%$ of the days supply of the last fill.

\section{- Augmentation}

Augmentation refers to a situation where an initial regimen is modified by adding another agent of a different class or insulin. Addition of another oral hypoglycemic agent was defined as starting a non-index medication belonging to a class other than the index prescription, while obtaining a refill for the index drug within a specified window [index drug fill date $+2.5 *$ (days supply of index drug fill)] and at least one more prescription for each of the drug classes in the follow up period. [Patients on initial poly-therapy were 
initially excluded to facilitate the distinction between augmentation and initial polytherapy]

\section{- Switching}

Agent switching was defined as starting a different class of oral hypoglycemic than the index prescription while not obtaining a refill for the index drug in the follow up period.

\section{- Non-modification}

Modification in medication regimen may indicate either augmentation or switching. Patients who experience no modification in their medication regimens for the entire follow up period (except discontinuers) were designated as non-modifiers. Dose changes were not classified as modification. Also changing specific drugs within the same class of oral hypoglycemic was not defined as modification.

If patients could not be categorized in any of the above patterns, then their prescription patterns were manually inspected to determine their status. Also, the outpatient pharmacies do not record prescriptions dispensed during an inpatient admission. These patients may be incorrectly classified as discontinuers due to the lack of prescription refill information during the length of their hospitalization. Hence, during the time spent in the hospital it was assumed that the medications are supplied by the hospital, and therefore the numbers of days of hospitalization were added as days supply in calculating drug utilization patterns. 


\section{Adherence with oral hypoglycemics}

Patients initiating either mono-therapy or poly-therapy involving insulin were excluded from the computation of adherence indices. For the rest of the patients who may have insulin use in the form of a switch or augmentation, all their insulin prescriptions were excluded from the calculation of adherence indices. This exclusion criterion was used, as only adherence to oral hypoglycemic agents was computed in the study. Also utilization of insulin through an administrative dataset is difficult to compute due to frequent changes in dosage instructions without proper documentation. Medication Possession Ratios ${ }^{150}$ were used as proxies for measurement of drug adherence in the study. Two different indices were computed and referred to as MPR-1 and MPR-2.

\section{- Medication Possession Ratio-1 (MPR-1)}

The Medication Possession Ratio-1 (MPR-1) was a proxy measure of medication adherence in the period between the first and last prescription fills. It is defined as the sum of days supply for all fills divided by the number of days of therapy between the first and last fills plus days supply for the last fill.

\section{MPR-1 = $\quad$ Sum of days supply}

(Number of days between the first and last fills) + days supply of the last fill

To account for possible early refills, any excess in the days supply due to early refill in the previous interval was allocated to the following interval.

This index assesses the proportion of days during the treatment that the patient possessed an adequate quantity of medication. Since the denominator of the ratio is the 
period during which the patient is in possession of medication, this measurement of adherence does not capture adherence as a consequence of premature discontinuation of therapy.

Also, as MPR-1 cannot be computed for patients not filling more than one prescription, these indices were not computed for patients who discontinue their oral hypoglycemic agents. The above formula for MPR-1 can be easily applied to nonmodifiers. However, if there are drugs from multiple classes consumed over the time period (i.e for switchers, augmenters, and those on initial poly-therapy) then MPR-1 should be computed separately for each class of drug. This weighted MPR-1 value was termed as Reg MPR-1 and was computed as follows:
Sum of $\{$ MPR-1 * [(Number of days between the first and last fills) days $\operatorname{Reg}$ MPR-1 = supply of the last fill]\} for all drugs
Sum of (Number of days between the first and last fills) + days supply of the last fill) for all drugs

If the admit and discharge dates of hospitalizations are included between the first and last fill dates of a particular class of drug, then the number of days of hospitalizations was added to the days supply of drugs for that particular class.

The formulas for MPR-1 and Reg MPR-1 mentioned above are restricted to computing adherence between two prescription refills. In addition, adherence indices were computed for the period between the index prescription and the end of the follow up period. In the first case, all gaps are embedded within a series of fills. In the second case, a terminal gap may be present after the last refill. Both measures assume that the gaps are due to reduced adherence rather than due to clinician instructions for temporary 
(in the case of "embedded gaps") or permanent (in the case of "terminal gaps") drug cessation.

When computing adherence indices using the end of follow up period as the end point instead of last refill, all the patients including discontinuers were used in the analysis. Here, the assumption made would be the fact that discontinuation is because of failure to comply and not due to control by diet and exercise. These adherence indices were denoted as MPR-2 and included discontinuers in their computation as opposed to MPR-1 and Reg MPR-1 wherein all the discontinuers were excluded.

\section{- Medication Possession Ratio-2 (MPR-2)}

The MPR-2 is a proxy measure of medication adherence in the period between the first prescription and end of the follow up period. It is defined as the sum of days supply for all fills divided by the number of days in the follow up period (i.e 365 days).

MPR-2 $=\frac{\text { Sum of days supply }}{\text { Number of days in the follow up period (i.e } 365 \text { days) }}$

To account for possible early refills, any excess in the days supply due to early refill in the previous interval was allocated to the following interval.

The above formula for MPR-2 can be easily applied to non-modifiers and switchers. However, if there are drugs from multiple classes consumed over the time period (i.e for augmenters, and those on initial poly-therapy) then MPR-2 should be computed separately for each class of drug. However, these rules might differ a bit for MPR-2. Reg MPR-2 was computed as follows: 
Sum of $\{$ MPR-2* [(Number of days between the first fill and end of follow up period) for all drugs\}

Reg MPR-2 = Sum of (Number of days between the first fill and end of follow period) for all drugs

If the admit and discharge dates of hospitalizations are included between the first fill date and end of follow up period for a particular class of drug, then the number of days of hospitalizations is added to the days supply of drugs for that particular class.

\section{Computation of costs}

- Total health care costs were computed irrespective of the diagnoses codes. The components of outpatient, emergency room (ER), hospitalizations, and prescription costs were separately computed.

- Diabetes related costs were computed by summing paid amounts for claims with a primary or secondary diagnosis code for type 2 diabetes. Prescriptions for oral hypoglycemics and insulin were identified on basis of National Drug Classification (NDC) Code. Diabetic supplies such as syringes, needles, and glucose testing equipment were also identified using NDC codes.

- In case of outpatient, prescription, and ER costs, costs were computed from Medicaid's perspective using the amount paid by Medicaid for each claim. In case of claims for hospitalizations, the amount reimbursed by Medicaid for each claim was not included in the dataset. Hence, average 2001 West Virginia Medicaid reimbursement rates on the basis of Diagnosis Related Groups (DRG) were used for computation of hospitalization costs. For DRG codes that did not have a reimbursement value from WV Medicaid, the relative weight of the DRG 
was used to assign hospitalization costs. On the basis of these averages, the average reimbursement assigned per unit DRG weight $=\$ 4,632.66$ per unit weight.

The above section discussed in detail the measurement of independent and dependent variables examined in the study. The following sections will describe the methodology and statistical methods used to meet each specific study objective:

\section{Objective 3}

\section{Patterns of oral hypoglycemic use}

Univariate analyses such as chi-squares were used to examine the differences in patterns of oral hypoglycemic (i.e non-modification, switching, augmentation, or discontinuation) between patients with and without depression. In addition, as the dependent variable had four possible categories, multivariate multinomial logistic regression techniques were used to estimate the impact of preexisting depression on the pattern of oral hypoglycemic use controlling for other confounding factors. A Multinomial logistic regression model simultaneously estimates binary logistic regression models for all possible comparisons among the outcomes categories, which enforces the logical relationship among the parameters and uses the data more efficiently. ${ }^{151}$ Patients receiving insulin therapy alone or in combination with oral hypoglycemics at the start of treatment were excluded from this analysis.

Pattern (non-modification, switching, augmentation, discontinuation) = constant

$+\beta($ Preexisting depression + Demographics + Drug regimen and complexity + Co-morbidity + Diabetes severity + Interaction with health care providers) + error term 


\section{Objective 4}

Secondary failure with oral hypoglycemic therapy

Augmenting or switching from the initial class of oral hypoglycemic agent is indicative of secondary failure with initial pharmacotherapy. A chi-square test statistic was used to examine the differences in secondary failure with initial oral hypoglycemic medication between patients with and without depression. A multivariate logistic regression analysis was used to examine the impact of preexisting depression on secondary failure controlling for other confounding factors. Patients who discontinued the use of oral hypoglycemic agents were excluded from the analysis.

\section{Secondary failure $(\mathrm{Yes} / \mathrm{No})=$ constant $+\beta($ Preexisting depression + Demographics + Drug regimen and complexity + Co-morbidity + Diabetes severity + Interaction with health care providers) + error term}

In addition, survival analysis techniques were used to estimate the impact of preexisting depression on time to secondary failure. As the outcome in this scenario is time-to-event data, one encounters the issue of censoring where the response is not observed for a portion of the subjects due to lack of follow-up time. Also, the frequency distribution of the response measurements is not normally distributed. Due to these characteristics of time-to-event data, the standard statistical methods are not appropriate for estimation. Univariate Kaplan Meier survival analysis and Cox proportional hazard models controlling for confounding factors were used for estimation of such data. Proportions hazards regression is a semi parametric model wherein the regression coefficients are estimated using partial likelihood procedures. ${ }^{152}$ 
Mathematically, the Cox proportional hazard model can be written as:

$$
h(t)=h_{o}(t) * \exp \{\beta X\}
$$

Where the hazard function $\boldsymbol{h}(\boldsymbol{t})$ is determined by a set of predictor variables collectively represented by $\boldsymbol{X}$. Analogous to the intercept in an Ordinary Least Squares (OLS) model, the Cox model has a baseline hazard function $\left\{\boldsymbol{h}_{\boldsymbol{o}}(\boldsymbol{t})\right\}$ which is estimated non-parametrically. Thus, the model is essentially an OLS regression of the logarithm of the hazard on the variables $\boldsymbol{X}$ with the baseline hazard being the intercept that varies with time. The covariates act multiplicatively on the hazard at any point in time, thus leading to the important assumption of proportional hazards rates between levels of variables. Therefore, prior to a multivariate analysis each predictor variable was tested for proportionality. This assumption can be tested by plotting Kaplan Meier survival estimates versus time, for the different levels of a variable, and checking whether the curves are linear with a constant proportion between each of the levels. In addition to these graphical techniques, significance tests provided by statistical software STATA will also be used for testing proportional hazards assumptions.

Time to Secondary failure $=$ constant $+\beta(\quad$ Preexisting depression + Demographics + Drug regimen and complexity + Co-morbidity + Diabetes severity + Interaction with health care providers) + error term 


\section{Objective 5}

\section{Initiation of insulin therapy}

A chi-square test statistic was used to examine the differences in initiation of insulin therapy between patients with and without depression. A multivariate logistic regression analysis was used to estimate the impact of preexisting depression on initiation of insulin therapy controlling for other confounding factors.

$$
\text { Insulin use }(\text { Yes/no })=\text { constant }+\beta(\text { Preexisting depression }+
$$

\section{Demographics + Drug regimen and complexity + Co-morbidity + Diabetes severity +} Interaction with health care providers) + error term

In addition, survival analysis techniques were used to estimate the impact of preexisting depression on time to initiate insulin therapy. Univariate Kaplan Meier survival analysis and Cox proportional hazard models controlling for other factors were used for estimation.

$$
\text { Time to insulin use }=\text { constant }+\beta(\text { Preexisting depression }+
$$

Demographics + Drug regimen and complexity + Co-morbidity + Diabetes severity + Interaction with health care providers) + error term 


\section{Objective 6}

\section{Adherence with oral hypoglycemic therapy}

This objective was examined using different indices for adherence such as the Reg MPR-1 and Reg MPR-2. Univariate analyses such as t-tests were employed to examine differences in adherence between patients with and without depression.

Ordinary Least Squares (OLS) regression techniques were used to estimate the impact of preexisting depression on adherence with oral hypoglycemic medications controlling for other factors. If the distributions of the adherence indices were skewed then transformations such as log transformations were performed on the indices.

These adherence scores were also categorized to examine the extremities in adherence levels and to estimate the relative impact of extreme adherence behavior on outcomes. Univariate chi-square tests were used to examine differences in adherence. As multiple categories of adherence were created, multivariate multinomial logistic regression analysis was used to estimate the impact of preexisting depression on these categories of adherence controlling for other confounding factors. Patients receiving insulin therapy alone or in combination with oral hypoglycemics at the start of treatment were excluded from this analysis. As insulin use in the follow up period can be a consequence of decrease in adherence, it was not controlled in the model.

$$
\text { Adherence }=\text { constant }+\beta(\text { Preexisting depression }+ \text { Demographics }+
$$

Drug regimen and complexity + Co-morbidity + Diabetes severity + Interaction with health care providers) + error term 


\section{Objective 7}

\section{Utilization and costs associated with type 2 diabetes}

Claims including either a primary or secondary diagnosis code for type 2 diabetes and prescriptions for insulin, oral hypoglycemics and diabetic supplies were identified in assessing total resource utilization associated with type 2 diabetes. The costs were also examined separately in terms of hospitalization/ER costs, outpatient costs, and pharmacy costs.

Parametric and non-parametric univariate tests such as t-tests and Mann-Whitney $\mathrm{U}$ tests were used depending on the distribution of the dependent variables. Ordinary Least Squares (OLS) regression techniques were used for the estimation of the impact of preexisting depression on total costs associated with type 2 diabetes controlling for other confounding factors. As the patients were selected over the period of a few years, the costs were inflated to 2002 costs using the medical care and prescription price index. ${ }^{153}$ If the distributions of the costs were skewed then transformations such as $\log$ transformations were performed to normalize the data.

In case of semi-log OLS models, empirically researchers have misinterpreted the estimates of the coefficients of the dummy variables by assuming that the parameter is estimated with no uncertainty. The coefficient of the primary independent variable was estimated using the correction of Halverson and Palmquist with a modification by Kennedy. ${ }^{154,155}$ This implies that an estimate for the coefficient of the dummy variable is Corrected Estimate $=\left\{\right.$ Exp (original estimate $\left.-0.5(\text { Standard error of estimate })^{2}\right\}-1$ Estimates from semi-log OLS models also need to interpreted in terms of dollar values. However, one cannot simply simulate costs by taking the anti-log of the fitted values. 
This value is subjected to retransformation bias, which is addressed using a smearing estimator. This estimate for semi-log OLS models is generally the mean of the anti-log of the residuals, which is multiplied by the fitted value to correct it for retransformation bias.

In models where the dependent variables are specific costs such as those due to ER/hospitalization visits and outpatient costs, there is a possibility of null values for the dependent variable for some of the observations. As a logarithmic transformation cannot be employed, a two-part model was used in this case to estimate the impact of depression on ER/hospitalization and outpatient costs. An initial multivariate logistic regression model was used to estimate the probability of having an ER/hospitalization or outpatient visit and a subsequent semi-logarithmic OLS model was conducted only on those patients incurring these specific costs in the follow up period.

Regression models will be analyzed to examine the impact of preexisting depression on events such as number of ER/hospitalization visits, and outpatient visits. As the dependent variable in this case is count data, the use of OLS can result in inefficient, inconsistent, and biased estimates. Hence, a Poisson regression analysis was conducted to meet these objectives. In a Poisson regression, the probability of a count is determined by a Poisson distribution, which has the defining characteristic that the conditional mean of the outcome is equal to the conditional variance. However, if the models were overdispersed (i.e the variance being greater than the mean) with a high proportion of zero counts, a negative binomial model was used for estimation. 
Type 2 diabetes related events and expenditures $=$ constant +

$\beta($ Preexisting depression + Demographics + Co-morbidity + Diabetes

severity + Interaction with health care providers) + error term

\section{Objective 8}

\section{Overall utilization and health care costs}

All medical and prescription claims in the 12-month follow up period were identified for this analysis, irrespective of diagnosis codes. The costs were also examined separately in terms of hospitalization/ER costs, outpatient costs, and pharmacy costs. Ordinary Least Squares (OLS) regression analysis was used for the estimation of the impact of preexisting depression on total health care costs. Regression models were also used to examine the impact of preexisting depression on events such as number of ER/hospitalization visits, outpatient visits, or prescriptions. Appropriate econometric methods as explained in objective 7 were used to estimate the impact of preexisting depression on overall utilization and health care costs.

Overall health care events and expenditures $=$ constant + $\beta($ Preexisting depression + Demographics + Co-morbidity + Diabetes severity + Interaction with health care providers) + error term 


\section{Objective 9}

Causal pathways between depression, adherence, and type 2 diabetes outcomes

Mediation analysis was conducted to meet this objective using the criteria suggested by MacKinnon. ${ }^{156}$ The following three equations were estimated to identify whether adherence with oral hypoglycemics mediates the relationship between depression and type 2 diabetes outcomes.

\section{$\underline{\text { Equation } 1}$}

Type 2 diabetes related events and expenditures $=$ constant + (a) Preexisting depression + Demographics + Co-morbidity + Diabetes severity + Interaction with health care providers + error term 1

\section{Equation 2}

Type 2 diabetes related events and expenditures $=$ constant +

(B) Preexisting depression + Adherence + Demographics + Co-morbidity +

Diabetes severity + Interaction with health care providers + error term 2

\section{Equation 3}

Adherence $=$ constant + Preexisting depression + Demographics + Comorbidity + Diabetes severity + Interaction with health care providers + Complexity of regimen + error term 3 
The following four steps can establish mediation:

Step 1: If depression is a significant predictor of outcomes in equation 1

Step 2: If depression significantly predicts adherence in equation 3

Step 3: If adherence significantly predicts outcomes while controlling for depression in equation 2. It is not sufficient to show that adherence and outcomes are correlated as they may be correlated because they are both caused by depression

Step 4: To establish that adherence completely mediates the relationship between depression and outcomes, the effect of depression on outcomes controlling for adherence in equation 2 (i.e. $\beta$ ) should be zero.

If all the four steps are met then the data is consistent with the hypothesis that adherence completely mediates the relationship between depression and outcomes, and if the first three steps are met but the fourth step is not met, them partial mediation is indicated. The presence of mediation will be tested by comparing the estimated effects of depression on type 2 diabetes outcomes with and without controlling for adherence in the estimation models (i.e. $\alpha$ and $\beta$ ). These differences in the estimates will be statistically tested using a Hausman type statistic. ${ }^{157}$ 


\section{CHAPTER FOUR}

\section{RESULTS AND DISCUSSION}

Previous chapters provided an overview of the issue of depression in patients with type 2 diabetes and discussed the available literature in this area. The specific objectives and the conceptual framework of the study were outlined. Data sources and detailed methodology employed to meet the study objectives were also provided. This chapter presents the results and discusses the findings of each study result. The results chapter is sub-divided on the basis of the two phases of the study.

\section{Phase 1}

\section{Results for Objective 1}

To estimate the prevalence of co-morbid depression in patients with type 2 diabetes

Patients in West Virginia Medicaid with a diagnosis of type 2 diabetes in the year 2000 were identified based on the specified selection criteria. Among these patients with type 2 diabetes, presence of co-morbid depression was identified based on the selection criteria for depression.

\section{Patients with type 2 diabetes:}

- Number of patients with continuous Medicaid eligibility and HMO non-eligibility between the ages of 18 and 64 in the year 2000: 10,742

- Number of type 2 diabetes patients who had co-morbid depression in 2000: 2,644 $(24.60 \%)$ 
Number of type 2 diabetic patients with

Prevalence rate $=\quad$ co-morbid depression during the year $2000 \times 100,000$

per 100,000

Number of patients with type 2 diabetes

during the year 2000

Prevalence rate $=\underline{2644 \times 100,000}=24,613.66$

per $100,000 \quad \frac{2644 \times 142}{100}$

In addition, a group of Medicaid enrollees without type 2 diabetes diagnosis or prescription for oral hypoglycemic agent was identified in the year 2000. Presence of co-morbid depression was also determined for this group of patients.

\section{Patients without type 2 diabetes:}

- Number of patients with continuous Medicaid eligibility and HMO non-eligibility between the ages of 18 and 64 in the year 2000: 9,364

- Number of patients without type 2 diabetes who had co-morbid depression in 2000: $1,911(20.40 \%)$

A Chi-square value of 50.50 was found to be significant $(p=0.001)$ indicating that co-morbid depression was more prevalent in patients with type 2 diabetes as compared to Medicaid enrollees without type 2 diabetes. However, this effect could also be a result of confounders such as demographics and presence of co-morbid conditions. Hence, a multivariate logistic regression was also performed controlling for age, gender, race, and co-morbidities. To separate the effects of type 2 diabetes and the presence of related comorbidities on the prevalence of depression, a block-wise logistic regression model was created consisting of the following blocks of co-variates entered sequentially in the model. 
Block 1: Demographic variables: age, gender, race, interaction term of age and diabetes, interaction term of gender and diabetes.

Block 2: Presence of chronic co-morbidities unrelated to type 2 diabetes: Cancer, asthma, and ulcer.

Block 3: Number of physician office visits and presence of chronic co-morbidities related to type 2 diabetes (Cardiovascular conditions, cerebro-vascular conditions, nephropathy, retinopathy, neuropathy and endocrine/metabolic disorders).

All three logistic regression models were found to be significant and the model results are summarized in Tables 1A-1C. Use of blocks of covariates shows that prevalence of depression in patients with type 2 diabetes was significantly higher than patients without type 2 diabetes after controlling for demographic characteristics such as gender, age, race, and their interactions (Odds Ratio $=1.259 ; \mathrm{p}=0.000$ ). The association remained significant after additionally controlling for presence of major chronic conditions unrelated to type 2 diabetes (Odds Ratio $=1.181 ; \mathrm{p}=0.000$ ). However, presence of type 2 diabetes did not retain its statistically significant impact on presence of co-morbid depression when presence of chronic conditions related to type 2 diabetes and number of physician office visits were controlled in the model (Odds Ratio $=0.924 ; \mathrm{p}$ $=0.089$ ). 
Table 1A: Logistic regression model to examine the relationship between type 2 diabetes and presence of co-morbid depression (Model 1)

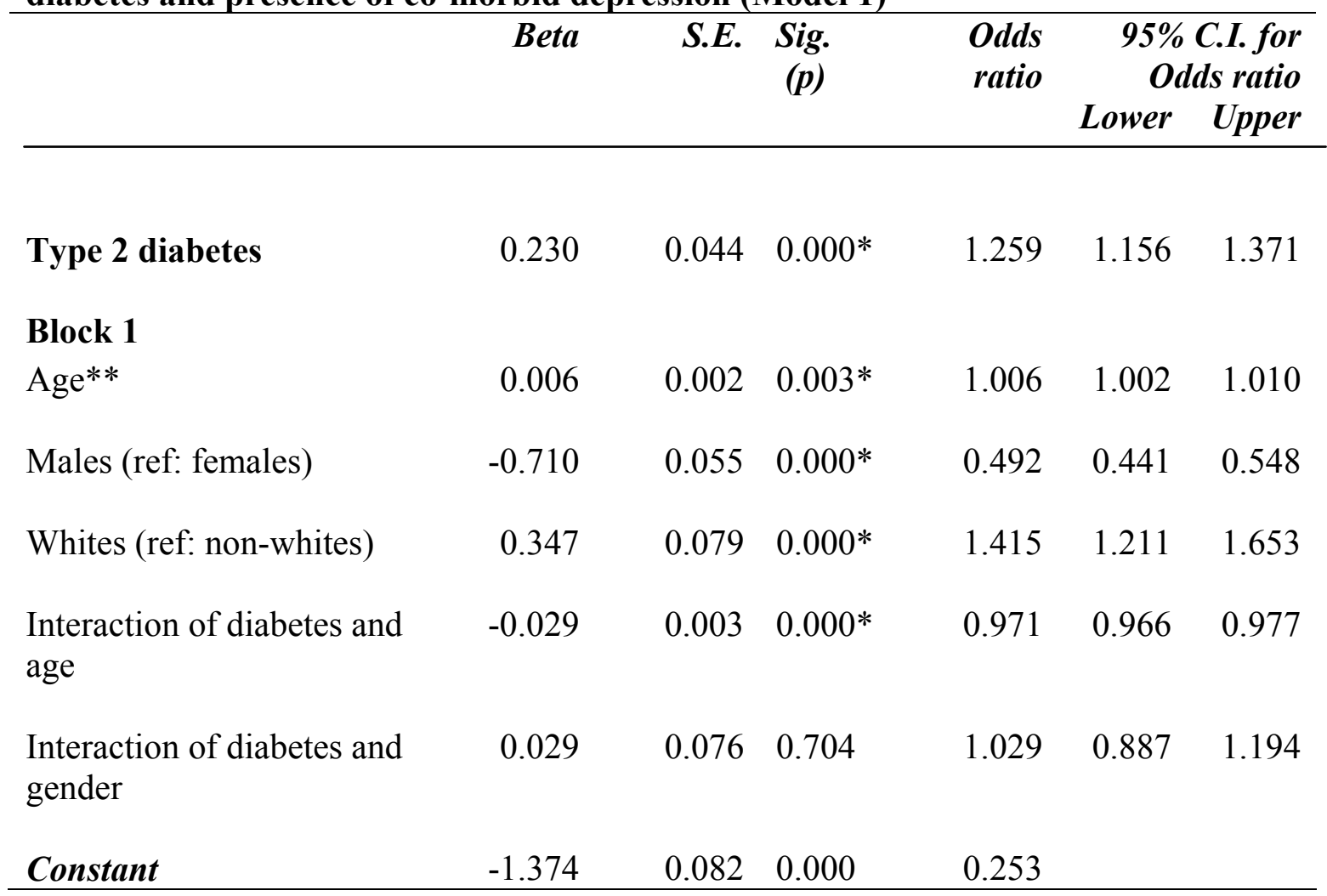

*significance at the 0.05 level

**Age is centered at the average value of age for the sample (40 years)

\section{Model fit statistics:}

Pseudo R-square $=4.1 \%$

-2 Log Likelihood $=20411.304 ; \chi^{2}=532.630, p=0.000$

Hosmer and Lemeshow's goodness of fit: $\chi^{2}=41.850 ; p=0.000$ 
Table 1B: Logistic regression model to examine the relationship between type 2 diabetes and presence of co-morbid depression (Model 2)

$\begin{array}{llllr}\text { Beta } & \text { S.E. } & \text { Sig. } & \text { Odds } & 95 \% \text { C.I. for } \\ & \text { (p) } & \text { ratio } & \begin{array}{r}\text { Odds ratio } \\ \text { Lower }\end{array} & \text { Upper }\end{array}$

$\begin{array}{lllllll}\text { Type } 2 \text { diabetes } & 0.174 & 0.044 & 0.000 * & 1.190 & 1.092 & 1.298\end{array}$

Block 1

\begin{tabular}{lcccccc} 
Age** & 0.003 & 0.002 & 0.188 & 1.003 & 0.999 & 1.007 \\
Males (ref: females) & -0.678 & 0.056 & $0.000^{*}$ & 0.507 & 0.455 & 0.566 \\
Whites (ref: non-whites) & 0.333 & 0.080 & $0.000^{*}$ & 1.396 & 1.193 & 1.633 \\
$\begin{array}{l}\text { Interaction of diabetes and } \\
\text { age }\end{array}$ & -0.027 & 0.003 & $0.000^{*}$ & 0.973 & 0.967 & 0.979 \\
$\begin{array}{l}\text { Interaction of diabetes and } \\
\text { gender }\end{array}$ & 0.038 & 0.076 & 0.622 & 1.038 & 0.894 & 1.206 \\
$\begin{array}{l}\text { Block 2 } \\
\text { Asthma }\end{array}$ & & & & & & \\
Cancer & 0.652 & 0.042 & $0.000^{*}$ & 1.919 & 1.767 & 2.083 \\
Ulcers & 0.139 & 0.077 & 0.071 & 1.149 & 0.988 & 1.336 \\
Constant & 0.555 & 0.075 & $0.000^{*}$ & 1.742 & 1.505 & 2.016 \\
\hline
\end{tabular}

*significance at the 0.05 level

$* *$ Age is centered at the average value of age for the sample (40 years)

\section{Model fit statistics:}

Pseudo R-square $=6.4 \%$

-2 Log Likelihood $=20098.353 ; \chi^{2}=845.581, \mathrm{p}=0.000$

Hosmer and Lemeshow's goodness of fit: $\chi^{2}=29.280 ; p=0.000$ 
Table 1C: Logistic regression model to examine the relationship between type 2 diabetes and presence of co-morbid depression (Model 3)

\begin{tabular}{|c|c|c|c|}
\hline \multirow[t]{2}{*}{ Beta } & \multirow[t]{2}{*}{$\begin{array}{ll}\text { S.E. } & \text { Sig. } \\
& (p)\end{array}$} & \multirow[t]{2}{*}{$\begin{array}{c}\text { Odds } \\
\text { ratio }\end{array}$} & $\begin{array}{r}95 \% \text { C.I. for } \\
\text { Odds ratio }\end{array}$ \\
\hline & & & Lower Upper \\
\hline
\end{tabular}

Type 2 diabetes

$\begin{array}{llllll}-0.072 & 0.047 & 0.124 & 0.931 & 0.850 & 1.020\end{array}$

Block 1

\begin{tabular}{|c|c|c|c|c|c|c|}
\hline Age** & -0.004 & 0.002 & 0.067 & 0.996 & 0.992 & 1.000 \\
\hline Males (ref: females) & -0.598 & 0.057 & $0.000 *$ & 0.550 & 0.492 & 0.614 \\
\hline Whites (ref: non-whites) & 0.328 & 0.081 & $0.000 *$ & 1.388 & 1.185 & 1.626 \\
\hline $\begin{array}{l}\text { Interaction of diabetes and } \\
\text { age }\end{array}$ & -0.022 & 0.003 & $0.000 *$ & 0.978 & 0.972 & 0.984 \\
\hline $\begin{array}{l}\text { Interaction of diabetes and } \\
\text { gender }\end{array}$ & 0.001 & 0.077 & 0.991 & 1.001 & 0.860 & 1.165 \\
\hline \multicolumn{7}{|l|}{ Block 2} \\
\hline Asthma & -0.111 & 0.079 & 0.164 & 0.895 & 0.766 & 1.046 \\
\hline Cancer & 0.350 & 0.077 & $0.000 *$ & 1.419 & 1.221 & 1.649 \\
\hline Ulcers & 0.483 & 0.043 & $0.000 *$ & 1.621 & 1.489 & 1.764 \\
\hline \multicolumn{7}{|l|}{ Block 3} \\
\hline Diabetic complications & 0.457 & 0.049 & $0.000 *$ & 1.579 & 1.434 & 1.738 \\
\hline $\begin{array}{l}\text { Number of physician office } \\
\text { visits }\end{array}$ & 0.040 & 0.003 & $0.000 *$ & 1.041 & 1.036 & 1.046 \\
\hline Constant & -1.971 & 0.090 & 0.000 & 0.139 & & \\
\hline
\end{tabular}

*significance at the 0.05 level

$* *$ Age is centered at the average value of age for the sample (40 years)

Model fit statistics:

Pseudo R-square $=9.5 \%$

-2 Log Likelihood $=19678.563 ; \chi^{2}=1265.371, \mathrm{p}=0.000$

Hosmer and Lemeshow's goodness of fit: $\chi^{2}=21.570$; $p=0.006$ 


\section{Discussion for Objective 1}

Our results demonstrated that $24.6 \%$ of type 2 diabetes patients in West Virginia Medicaid had co-morbid depression. Previous studies have found depression prevalence rates ranging from $6.1 \%$ to $49.3 \%$ in patients with type 2 diabetes. ${ }^{29}$ The populationbased study conducted in KPNW had a prevalence rate of $17.6 \% .{ }^{139}$ Our rates may be higher due to an indigent and younger population with a higher proportion of females. The association of type 2 diabetes and co-morbid depression was significant after controlling for confounders indicating that patients with type 2 diabetes have higher odds of co-morbid depression as compared to patients without type 2 diabetes. However, when the logistic regression model was reanalyzed with the presence of type 2 diabetes related co-morbidities as independent variables, the odds of co-morbid depression were not significantly different for patients with type 2 diabetes as compared to patients without type 2 diabetes. These results are interesting in the fact that they suggest that higher observed rates of co-morbid depression in patients with type- 2 diabetes are primarily due to the higher number of co-morbid conditions associated with type 2 diabetes. However, one can attribute these co-morbid conditions and subsequent depression to type 2 diabetes and thus reasonably conclude that type 2 diabetes is associated with a higher prevalence of co-morbid depression. Similar results were obtained in a study by Pouwer and associates, ${ }^{138}$ which suggested that the prevalence of co-morbid depression is higher in patients with type 2 diabetes and co-morbid disease(s), but not in patients with type 2 diabetes only. A variety of other studies have found that while medical conditions in general are risk factors for psychological disturbance, type 2 diabetes carries no unique risk. ${ }^{121}$ The comprehensive review by Anderson and associates 
contained two studies that compared rates of depression between people with diabetes and those with other medical conditions; neither found a significant difference. ${ }^{29}$ These results suggest that factors involved in the general burden of illness contribute to the observed elevated prevalence of co-morbid depression in patients with type 2 diabetes.

\section{Results for Objective 2}

To estimate the incidence of type 2 diabetes in patients with pre-existing depression

Patients with depression were identified on the basis of the inclusion criteria for depression in the year 1997. As a comparison group, a group of Medicaid enrollees without depression diagnosis or prescription of antidepressants was identified in the year 1997. Patients with a medical claim for type 2 diabetes or a prescription for an oral hypoglycemic agent in the year 1997 were excluded from the analysis. These patients were then followed till December 31, 2002, to identify incident cases of type 2 diabetes in both the groups.

\section{Patients with depression:}

- Number of patients with a diagnosis of depression: 12,589

- Number of depressed patients without type 2 diabetes in 1997: 11,345

- Number of eligible patients (Medicaid eligible 97-2002; HMO non eligible 972002; age greater than or equal to 18 and age less than or equal to 60 as on January 1, 1998): 4,472

- Number of depressed patients who had incident type 2 diabetes in the follow-up period (1998-2002): $704(15.70 \%)$ 
Number of new cases of type 2 diabetes in patients with

Incidence rate $=$ preexisting depression occurring between 1998-2002 $\quad$ x 100,000

per 100,000 Number of patients with depression in 1997

$\begin{aligned} & \text { Incidence rate }= \\ & \text { per } 100,000\end{aligned} \frac{704 \times 100,000}{4,472}=15,742.39$

\section{Patients without depression:}

- Number of patients without a diagnosis of depression: 52,653

- Number of non-depressed patients without type 2 diabetes in 1997: 50,798

- Number of eligible patients (Medicaid eligible 97-2002; HMO non eligible 972002; age greater than equal to 18 and age less than equal to 60 as on January 1, 1998): 5,195

- Number of non-depressed patients who had incident type 2 diabetes in the followup period (1998-2002): 606(11.70\%)

A univariate chi-square analysis was performed to determine the association between the presence of pre-existing depression and incidence of type 2 diabetes patients. Approximately $15.7 \%$ of depressed patients developed type 2 diabetes as compared to $11.70 \%$ of non-depressed patients. A Chi-square value of 34.104 was found to be significant with a p-value of 0.000 . These numbers indicate that the incidence of type 2 diabetes was slightly higher in patients with pre-existing depression. However, this effect could also be a result of confounders such as demographics and presence of co-morbid conditions. Hence, a multivariate logistic regression was performed to determine the association of pre-existing depression and incidence of type 2 diabetes controlling for age, gender, race, and presence of cardio-vascular co-morbidities at baseline. 
The logistic regression model was found to be significant with a Nagelkerke Rsquare value of $7.10 \%$. The model results are summarized in Table 2. A significant adjusted odds ratio indicates that the association between presence of pre-existing depression and incidence of type 2 diabetes is still significant and that patients with preexisting depression are more likely to develop type 2 diabetes as compared to enrollees without pre-existing depression.

Due to the use of interaction effects, the odds of developing incident type 2 diabetes in patients with depression as compared to patients without depression needs to be computed separately for males and females. The interaction term for gender and presence of pre-existing depression was significant, suggesting that the odds of developing incident type 2 diabetes in patients with depression differs for males and females. The results of logistic regression estimate that a 40 year old female with preexisting depression is nearly one and a half times more likely to develop incident type 2 diabetes as compared to a 40 year old female without depression after controlling for age, gender, race, and cardiovascular co-morbidity at baseline. However, 40 year old males do not show a relationship between presence of pre-existing depression and development of incident type 2 diabetes (Odds Ratio for Females=1.479; Odds Ratio for Males $=0.997)$. 
Table 2: Logistic regression model to examine the relationship between pre-existing depression and incidence of type 2 diabetes

\begin{tabular}{|c|c|c|c|}
\hline \multirow[t]{2}{*}{ Beta } & \multirow[t]{2}{*}{$\begin{array}{cc}\text { S.E. } & \text { Sig. } \\
& \text { (p) }\end{array}$} & \multirow[t]{2}{*}{$\begin{array}{c}\text { Odds } \\
\text { ratio }\end{array}$} & $\begin{array}{l}95 \% \text { C.I. for } \\
\text { Odds ratio }\end{array}$ \\
\hline & & & Lower \\
\hline
\end{tabular}

\begin{tabular}{lcccccc} 
Depression & 0.392 & 0.086 & $0.000^{*}$ & 1.479 & 1.250 & 1.751 \\
Age** & 0.039 & 0.004 & $0.000^{*}$ & 1.040 & 1.032 & 1.048 \\
Males (ref: females) & -0.024 & 0.091 & 0.791 & 0.976 & 0.816 & 1.168 \\
Whites (ref: non-whites) & 0.135 & 0.157 & 0.389 & 1.145 & 0.841 & 1.558 \\
& -0.019 & 0.006 & $0.001^{*}$ & 0.981 & 0.969 & 0.993 \\
$\begin{array}{l}\text { Interaction of depression and } \\
\text { age }\end{array}$ & & & & & & \\
$\begin{array}{l}\text { Interaction of depression and } \\
\text { gender }\end{array}$ & -0.394 & 0.138 & $0.004^{*}$ & 0.674 & 0.515 & 0.884 \\
$\begin{array}{l}\text { Cardiovascular co-morbidity } \\
\text { Constant }\end{array}$ & 0.760 & 0.067 & $0.000^{*}$ & 2.139 & 1.877 & 2.438 \\
\hline
\end{tabular}

*significance at the 0.05 level

$* *$ Age is centered at the average value of age for the sample (40 years)

\section{Model fit statistics:}

Pseudo R-square $=7.1 \%$

-2 Log Likelihood $=7003.420 ; \chi^{2}=370.605, \mathrm{p}=0.000$

Hosmer and Lemeshow's goodness of fit: $\chi^{2}=7.142 ; p=0.521$ 
Table 3: Logistic regression model to examine the relationship between pre-existing depression and incidence of type 2 diabetes (excluding cases of incident type 2 diabetes in the first 2 years of follow up)

\begin{tabular}{|c|c|c|c|c|}
\hline \multirow[t]{2}{*}{ Beta } & \multirow[t]{2}{*}{ S.E. } & \multirow[t]{2}{*}{$\begin{array}{l}\text { Sig. } \\
\text { (p) }\end{array}$} & \multirow[t]{2}{*}{$\begin{array}{l}\text { Odds } \\
\text { ratio }\end{array}$} & $\begin{array}{l}95 \% \text { C.I. for } \\
\text { Odds ratio }\end{array}$ \\
\hline & & & & Lower Upper \\
\hline
\end{tabular}

\begin{tabular}{lcccccc} 
Depression & 0.410 & 0.114 & $0.000^{*}$ & 1.507 & 1.205 & 1.884 \\
Age** & 0.030 & 0.005 & $0.000^{*}$ & 1.030 & 1.019 & 1.041 \\
Males (ref: females) & 0.078 & 0.121 & 0.519 & 1.081 & 0.853 & 1.371 \\
Whites (ref: non-whites) & 0.212 & 0.213 & 0.320 & 1.236 & 0.814 & 1.875 \\
$\begin{array}{l}\text { Interaction of depression and } \\
\text { age }\end{array}$ & -0.011 & 0.008 & 0.155 & 0.989 & 0.974 & 1.004 \\
$\begin{array}{l}\text { Interaction of depression and } \\
\text { gender }\end{array}$ & -0.371 & 0.179 & $0.038^{*}$ & 0.690 & 0.486 & 0.979 \\
$\begin{array}{l}\text { Cardiovascular co-morbidity } \\
\text { Constant }\end{array}$ & 0.704 & 0.088 & $0.000^{*}$ & 2.022 & 1.703 & 2.401 \\
\hline
\end{tabular}

*Significance at the 0.05 level

**Age is centered at the average value of age for the sample (40 years)

\section{Model fit statistics:}

Pseudo R-square $=4.4 \%$

-2 Log Likelihood $=4592.426 ; \chi^{2}=164.520, p=0.000$

Hosmer and Lemeshow's goodness of fit: $\chi^{2}=11.245 ; p=0.188$ 


\section{Discussion for Objective 2}

Evidence from a few prospective studies indicates that depression doubles the risk of incident type 2 diabetes even after controlling for other risk factors. Controlling for multiple risk factors in relatively small samples, Eaton and associates ${ }^{32}$ and Kawakami and associates ${ }^{33}$ have shown that presence of depressive symptoms more than doubled the risk of developing type 2 diabetes. A couple of other studies have used data from the NHEFS ${ }^{34,35}$ to test the relationship between depression and incidence of diabetes. However, these studies using the NHEFS cohort were not specific to incidence of type 2 diabetes and had inconclusive results.

The study results indicated a significant interaction between gender and presence of pre-existing depression. Pre-existing depression was not significantly associated with incident type 2 diabetes in males; while females with pre-existing depression were one and a half times more likely to develop incident type 2 diabetes as compared to females without pre-existing depression controlling for demographics and presence of cardiovascular co-morbidity at baseline.

However, this impact of pre-existing depression on incidence of type 2 diabetes may be confounded by factors such as obesity. Being overweight is associated with onset of type 2 diabetes and depressed individuals suffer from problems with appetite and weight gain. One can also view obesity as a mediating variable in the relationship between depression and onset of type 2 diabetes. However, controlling for the presence of chronic medical conditions such as cardiovascular problems, cerebrovascular conditions and metabolic/endocrine disorders at baseline failed to attenuate the relationship between depression and incidence of diabetes. Another possible explanation 
for this relationship is the presence of detection bias as individuals with depressive symptoms may be more likely to seek medical care than individuals without symptoms. Therefore, they would be more likely to be diagnosed with diabetes than those without symptoms leading to an increased prevalence and incidence of type 2 diabetes among patients with depression. In this study, an attempt was made to reduce the bias by controlling for the number of physician office visits at baseline (year 1997). The presence of pre-existing depression maintained a significant impact on the incidence of type 2 diabetes after controlling for this ascertainment bias.

Another important limitation of the study is the problem of undetected type 2 diabetes. Diabetic disturbance in glucose metabolism may start 5-10 years before the diagnosis of type 2 diabetes. It is possible that symptoms due to the presence of undetected diabetes could have led to depression at baseline. Also, depressive symptoms may worsen glycemic control exclusively among those who already had diabetic metabolic disturbance. These factors can lead to an artificial effect of a higher incidence of type 2 diabetes in patients with depression as compared to patients without depression. To avoid this problem of undetected diagnosis, a sub-analysis was conducted by excluding cases of incident diabetes in the first two years of the follow-up period. The results of this sub-analysis were highly comparable to the original results confirming the higher incidence of type 2 diabetes in female patients with depression (Table 3).

Also, the peak onset of depression usually occurs during early adulthood between the ages of 20 and 30; while the onset of type 2 diabetes typically occurs much later. ${ }^{158,159}$ On the basis of this alone, one would expect depressive symptoms to precede the 
incidence of type 2 diabetes. Hence, a statistical interaction between presence of depression and age at baseline become highly important control variables in the model.

There is also the possibility of misclassification bias as there might be individuals at baseline who have depression symptomatology but have not sought treatment or medical attention and are hence classified as patients without depression. However, this misclassification would only lead to an underestimation of the effect of depression on incidence of type 2 diabetes. As significant results are obtained even with underestimation we can easily conclude that there is a significant positive relationship between presence of pre-existing depression and incidence of type 2 diabetes.

\section{Phase 2}

In phase 2 of the study, patients newly diagnosed with type 2 diabetes were identified. The date of the first prescription for an oral hypoglycemic agent was identified for these patients and treated as the index date for the study. Patients were then followed for a period of 12 months post index date.

\section{$\underline{\text { Sample selection }}$}

In the three-year enrollment period between January 1, 1998 and December 31, 2000, 27,676 patients with a medical diagnosis for type 2 diabetes were identified. Of these, 9,116 patients were newly diagnosed with type 2 diabetes in the enrollment period. Only about $50 \%$ of these newly diagnosed patients received any type of oral hypoglycemic prescription in the period between January 1, 1998 and December 31, 2002. Of these 4,459 newly diagnosed type 2 diabetes patients, 1,021 patients had preexisting depression and the remainder had no diagnosis of depression in the entire study 
period. After excluding patients on the basis of other criteria, the final cohort consisted of 1,326 type 2 diabetes patients (471 patients with depression and 855 patients without depression). A detailed algorithm of sample attrition for the cohort followed for 12 months post index date is provided in Figure 5. 
Figure 5: Algorithm for sample attrition of patients with newly diagnosed type 2 diabetes (12 month follow up period)

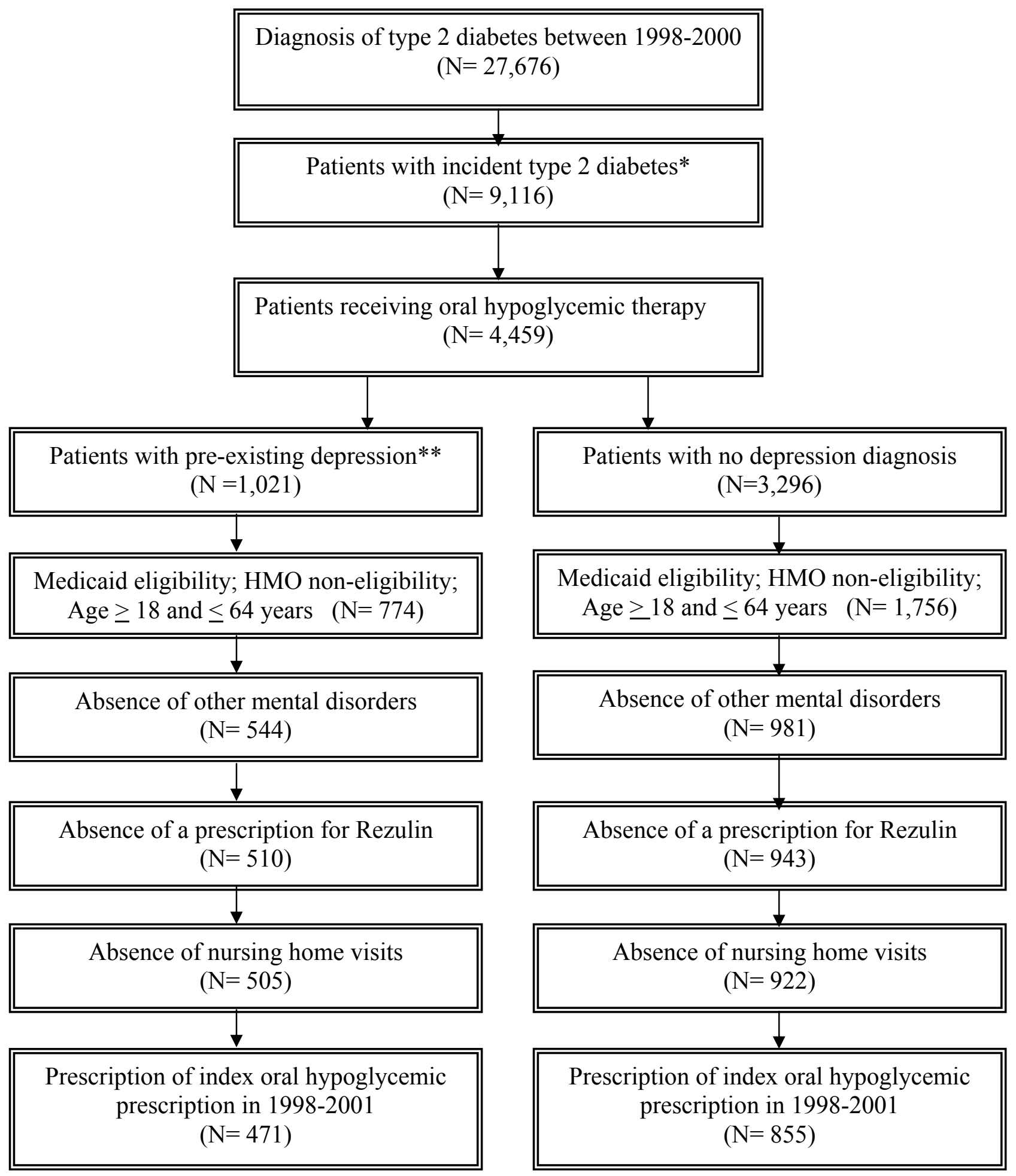

*Incident diabetes is identified by absence of diagnosis codes for diabetes or antidiabetic medications in the 12 month period prior to index date of diabetes in 98-2000; coupled with Medicaid eligibility and non-HMO eligibility in the 12 month pre-index date

**Pre-existing depression is identified by the presence of a depression diagnosis or use of an antidepressant in the period before incidence of type 2 diabetes 


\section{Baseline characteristics of newly diagnosed type 2 diabetes patients}

To meet the study objectives of examining differences in adherence and health care expenditures between patients with and without depression, a number of baseline demographic and clinical variables were controlled to prevent any bias in the validity of the study results. The comparison of these baseline characteristics between patients is provided in Table 4. The baseline characteristics were grouped into factors such as demographic characteristics, complexity of regimen, co-morbid conditions, provider interaction, and diabetes severity. A Mann Whitney U test was used to compare continuous variables, while a chi-square test was used to compare differences between categorical variables.

\section{Demographic characteristics:}

Patients with pre-existing depression were significantly younger as compared to patients without pre-existing depression. Mean age for patients with depression was 46.87 years $( \pm 9.35)$ as compared to a mean age of 49.12 years $( \pm 10.65)$ for patients without depression. A significantly higher percent of depressed patients were female (81.5\%) as compared to non-depressed patients (53.6\%). Depressed and non-depressed patients did not differ significantly on other demographic characteristics such as race, geographical location, and year of index prescription.

\section{Complexity of regimen}

Patients with depression consumed significantly higher number of pills per day for chronic medications $(3.72 \pm 3.62)$ as compared to non-depressed patients $(2.74 \pm 3.31)$. Depressed patients also filled a greater number of prescriptions for chronic medications $(11.63 \pm 10.07)$ as compared to non-depressed patients $(9.27 \pm 3.30)$. However, the two 
groups did not differ significantly in the number of pills per day for oral hypoglycemic agents.

\section{Co-morbid conditions}

Baseline co-morbid conditions other than depression were evaluated for patients with type 2 diabetes. Health care utilization variables identifying the presence and severity of co-morbid conditions (excluding those due to depression) such as total health care costs, number of ER/hospitalization episodes, and number of therapeutic classes of chronic medications indicated that patients with depression had a significantly higher presence of co-morbid conditions as compared to patients without depression. Scores on the Charlson co-morbidity index indicated greater co-morbidity in depressed patients $(0.76 \pm 1.22)$ as compared to non-depressed patients $(0.55 \pm 1.19)$. Also co-morbid conditions such as asthma, ulcer, and cardiovascular conditions occurred more frequently in depressed patients as compared to non-depressed patients.

\section{Provider interaction}

There were no differences in the fraction of patients enrolled in PAAS between depressed and non-depressed patients. However, a greater fraction of non-depressed patients (52.3\%) used a single pharmacy in the last six months as compared to depressed patients (38.9\%), implying greater pharmacy patronage for non-depressed patients.

\section{Diabetes severity}

As the study population consisted of a group of newly diagnosed type 2 diabetes patients, drastic differences in terms of diabetes severity were not expected. However, as our study index date was the date of the first prescription for an oral hypoglycemic, there could be potentially a gap between initiation of oral hypoglycemic therapy and initial 
diagnosis of type 2 diabetes thus leading to differences in diabetes severity. Baseline comparisons demonstrated that depressed and non-depressed patients did not have significant differences in diabetes severity as measured on the basis of type of prescribing physician, initiation on polytherapy, gap between diagnosis and therapy, and number of diabetes related ER/hospitalization episodes. The only differences were seen in terms of diabetes related costs in the pre 12-month period and the class of the index oral hypoglycemic agent. Depressed patients had significantly higher diabetes related costs $(\$ 899.35 \pm 3,275.30)$ than non-depressed patients $(\$ 629.42 \pm 2,751.92)$. Also, in terms of the class of index oral hypoglycemic agent, the most notable difference was that a higher fraction of depressed patients (2.8\%) were prescribed an alpha-glucosidase inhibitor as compared to non-depressed patients $(0.8 \%)$. Also, a slightly higher fraction of depressed patients $(2.5 \%)$ were prescribed meglitinides as compared to non-depressed patients (1.1\%).

Overall, the results demonstrated distinct differences between patients with and without pre-existing depression. Depressed patients were significantly younger and included a higher proportion of females. Depressed patients had a significantly higher number of co-morbid conditions and a more complex drug regimen at baseline. Level of diabetes severity was not too different between the two groups. However, differences in the index class of oral hypoglycemic agent was possibly indicative of a higher fraction of depressed patients being obese as compared to non-depressed patients. This was deduced on the basis of clinical information indicating that alpha-glucosidase and meglitinides are typically prescribed to obese patients as opposed to sulfonylurea due to the absence of any side effects of weight gain due to these medications. 
These baseline variables were used in the multivariate framework to estimate the impact of depression on type 2 diabetes outcomes adjusting for these baseline characteristics. The effect of the individual constructs such as demographics, comorbidity, provider interaction, complexity of regimen, and diabetes severity on type 2 diabetes outcomes were not an objective of the study. Hence, in all the results and discussions of study objectives, the effect of these baseline control variables on type 2 diabetes outcomes will not be discussed. Only the effect of depression on type 2 diabetes outcomes controlling for these baseline variables will be examined and discussed. 
Table 4: Baseline characteristics of newly diagnosed type 2 diabetes patients

\begin{tabular}{llcl}
\hline $\begin{array}{l}\text { Depressed } \\
(n=471)\end{array}$ & $\begin{array}{c}\text { Non-depressed } \\
(n=855)\end{array}$ & $\begin{array}{c}\text { Test } \\
\text { Statistic }\end{array}$ & $\begin{array}{l}\text { Sig. } \\
(p)\end{array}$ \\
\hline
\end{tabular}

\section{Demographic Characteristics}

Age (in years) $\quad 46.87 \pm 9.35^{*} \quad 49.12 \pm 10.65^{*} \quad \mathrm{M}=169467.00 \quad 0.000^{* *}$

Gender
- Males
$18.53 \%$
$46.63 \%$
$\chi^{2}=101.30$
$0.000^{* *}$
- Females
$81.47 \%$
$53.37 \%$

Race

- Whites $95.91 \%$

- Non-whites

$4.09 \%$

$93.64 \%$

$\chi^{2}=3.11$

0.078

$6.36 \%$

Residence

- Urban

$93.03 \%$

- Rural

$6.97 \%$

$92.05 \%$

$7.95 \%$

0.534

Year of index prescription
- 1998
$21.00 \%$
$25.32 \%$
$\chi^{2}=4.81$
0.186
- 1999
$32.11 \%$
$32.04 \%$
- 2000
$36.83 \%$
$35.14 \%$
- 2001
$10.06 \%$
$7.50 \%$

\section{Complexity of regimen}

Number of pills per day

$$
3.72 \pm 3.62 *
$$

$2.74+3.31 *$

$M=164214.00$

$0.000^{* *}$ for chronic medications

Number of prescriptions

$$
11.63 \pm 10.07 *
$$

$9.27 \pm 3.30^{*}$

$M=165250.00$

$0.000^{* *}$ for chronic medications

Number of pills per day

$$
1.49 \pm 0.73 *
$$

$$
1.44+0.69^{*}
$$

$M=195228.00$

0.301 
Table 4: Baseline characteristics of newly diagnosed type 2 diabetes patients (Contd.)

$\begin{array}{cccc}\begin{array}{c}\text { Depressed } \\ (n=471)\end{array} & \begin{array}{c}\text { Non-depressed } \\ (n=855)\end{array} & \begin{array}{c}\text { Test } \\ \text { Statistic }\end{array} & \begin{array}{c}\text { Sig. } \\ (p)\end{array}\end{array}$

\section{Baseline Co-morbid conditions}

\begin{tabular}{|c|c|c|c|c|}
\hline $\begin{array}{l}\text { Number of ER/ } \\
\text { hospitalizations } \\
\text { (Excluding those } \\
\text { due to depression) }\end{array}$ & $2.03 \pm 3.47^{*}$ & $1.09 \pm 2.16^{*}$ & $M=162948.00$ & $0.000^{* *}$ \\
\hline $\begin{array}{l}\text { Total health costs } \\
\text { (Excluding those } \\
\text { due to depression) }\end{array}$ & $\begin{array}{c}7,623.21 \\
+10,942.62 *\end{array}$ & $\begin{array}{r}5,318.42 \\
\pm 11,378.92 *\end{array}$ & $M=136320.00$ & $0.000 * *$ \\
\hline $\begin{array}{l}\text { Charlson } \\
\text { co-morbidity }\end{array}$ & $0.76 \pm 1.22 *$ & $0.55 \pm 1.19 *$ & $\mathrm{M}=177512.00$ & $0.000 * *$ \\
\hline $\begin{array}{l}\text { Number of } \\
\text { therapeutic classes } \\
\text { of medications }\end{array}$ & $2.86 \pm 1.90^{*}$ & $2.12 \pm 1.82^{*}$ & $M=153958.00$ & $0.000^{* *}$ \\
\hline $\begin{array}{l}\text { Presence of co-morbic } \\
\text { - Cardio-vascula } \\
\text { - Cancer } \\
\text { - Asthma } \\
\text { - Ulcers }\end{array}$ & $\begin{array}{r}\text { conditions } \\
64.10 \% \\
5.92 \% \\
21.24 \% \\
5.12 \%\end{array}$ & $\begin{array}{r}58.13 \% \\
3.91 \% \\
14.62 \% \\
1.83 \%\end{array}$ & $\begin{array}{l}\chi^{2}=4.55 \\
\chi^{2}=3.01 \\
\chi^{2}=9.42 \\
\chi^{2}=11.88\end{array}$ & $\begin{array}{l}0.033^{* *} \\
0.083 \\
0.002^{* *} \\
0.001^{* *}\end{array}$ \\
\hline
\end{tabular}

\section{Provider interaction}

PAAS enrollment

$12.74 \%$

$11.13 \%$

$\chi^{2}=0.78$

0.377

Number of pharmacies seen in last 12 months
- No pharmacy
$0.82 \%$
$7.05 \%$
- Single
$38.91 \%$
$52.22 \%$
- Multiple
$60.27 \%$
$40.73 \%$
$\chi^{2}=59.93 \quad 0.000^{*}$ 
Table 4: Baseline characteristics of newly diagnosed type 2 diabetes patients (Contd.)

\begin{tabular}{lccc}
$\begin{array}{l}\text { Depressed } \\
(n=471)\end{array}$ & $\begin{array}{c}\text { Non-depressed } \\
(n=855)\end{array}$ & $\begin{array}{c}\text { Test } \\
\text { Statistic }\end{array}$ & $\begin{array}{c}\text { Sig. } \\
(p)\end{array}$ \\
\hline
\end{tabular}

\section{Baseline diabetes severity}

Index oral hypoglycemic prescribed by:

\begin{tabular}{|c|c|c|c|c|}
\hline $\begin{array}{l}\text { - Endocrinologist } \\
\text { - Internal medicine } \\
\text { - Family practitioner }\end{array}$ & $\begin{array}{r}1.92 \% \\
31.53 \% \\
66.55 \%\end{array}$ & $\begin{array}{r}1.52 \% \\
30.14 \% \\
68.44 \%\end{array}$ & $\chi^{2}=0.70$ & 0.705 \\
\hline
\end{tabular}

\begin{tabular}{|c|c|c|c|c|}
\hline $\begin{array}{l}\text { Number of diabetes } \\
\text { related ER/hospitalizations }\end{array}$ & $0.21 \pm 0.59 *$ & $0.14 \pm 0.44^{*}$ & $M=194630.00$ & 0.080 \\
\hline $\begin{array}{l}\text { Total diabetes related } \\
\text { costs }\end{array}$ & $\begin{array}{r}899.35 \\
+3,275.30^{*}\end{array}$ & $\begin{array}{r}629.42 \\
+2,751.92^{*}\end{array}$ & $M=186703.00$ & $0.020^{* *}$ \\
\hline $\begin{array}{l}\text { Gap between diagnosis } \\
\text { and index prescription }\end{array}$ & $\begin{aligned} & 117.16 \\
\pm & 236.23 *\end{aligned}$ & $\begin{array}{r}97.29 \\
+214.15^{*}\end{array}$ & $\mathrm{M}=191107.50$ & 0.105 \\
\hline Initial polytherapy & $6.21 \%$ & $8.92 \%$ & $\chi^{2}=3.11$ & 0.078 \\
\hline $\begin{array}{l}\text { Index class of oral hypoglycen } \\
\text { - Sulfonylurea } \\
\text { - Thiazolidenedione } \\
\text { - Biguanides } \\
\text { - Alpha-glucosidase } \\
\text { - Sulfonylurea-biguanid } \\
\text { - Meglitinides }\end{array}$ & $\begin{array}{l}\text { emic } \\
49.72 \% \\
7.22 \% \\
35.00 \% \\
2.81 \% \\
\text { ide } 2.83 \% \\
2.42 \%\end{array}$ & $\begin{array}{r}55.02 \% \\
5.81 \% \\
34.60 \% \\
0.82 \% \\
2.72 \% \\
1.13 \%\end{array}$ & $\chi^{2}=14.40$ & $0.013 * *$ \\
\hline
\end{tabular}

*Mean \pm S.D.

**significance at the 0.05 level

M= Mann-Whitney U Test; $\chi^{2}=$ Chi-square test

PAAS = Physician Assured Access System 


\section{$\underline{\text { Results for Objective } 3}$}

To examine the impact of pre-existing depression on patterns of oral hypoglycemic use in new patients with type 2 diabetes

Patients initiating polytherapy or insulin therapy were excluded from this analysis. Univariate results for patterns of oral hypoglycemic therapy are presented in Table 5. Results indicated that a significantly higher fraction of depressed patients $(39.50 \%)$ discontinued oral hypoglycemic therapy as compared to non-depressed patients (32.90\%). Only $37.20 \%$ of depressed patients non-modified their index oral hypoglycemic therapy as compared to $50.90 \%$ of non-depressed patients who remained on their original therapy for the entire 12 month follow up period. Of the patients who modified their index oral hypoglycemic therapy, 9.20\% depressed patients switched therapy as compared to $6.30 \%$ non-depressed patients. The fraction of patients augmenting their index therapy by adding another oral hypoglycemic agent or insulin to their drug regimen was also significantly higher for depressed patients (14.1\%) as compared to non-depressed patients (9.9\%). A univariate chi-square value of 22.95 was found to be significant with a p-value of 0.000 . These numbers indicate that the patterns of oral hypoglycemic use were significantly favorable for non-depressed patients indicating better management of type 2 diabetes.

However, this effect could also be a result of confounders such as demographics, co-morbidity, diabetes severity, complexity of regimen, and interaction with health care providers. Hence, a multivariate multinomial logistic regression was performed to determine the impact of depression on patterns of oral hypoglycemic use controlling for 
baseline confounding factors. Non-modification was used as the reference category for all comparisons in the multinomial logistic model framework.

The multinomial logistic regression model was found to be significant with a Nagelkerke R-square value of $16.1 \%$. The model results are summarized in Table 6A6C. Results of the multivariate model indicated that controlling for confounding covariates, patients with depression were nearly one and a half times more likely to discontinue oral hypoglycemic therapy in the 12 month follow up period as compared to patients without depression $(\mathrm{p}=0.004)$. Results also demonstrated that depressed patients were 1.7 times more likely to switch $(\mathrm{p}=0.046)$ and two times more likely to augment therapy $(\mathrm{p}=0.003)$ as compared to patients without depression. 
Table 5: Pattern of use of oral hypoglycemics in the 12-month follow up period

\begin{tabular}{|c|c|c|c|c|c|}
\hline & $\begin{array}{c}\text { Depressed } \\
(n=446)\end{array}$ & $\begin{array}{c}\text { Non-depressed } \\
(n=791)\end{array}$ & $\begin{array}{c}\text { Total } \\
(n=1237)\end{array}$ & $\begin{array}{c}\text { Test } \\
\text { statistic }\end{array}$ & $\begin{array}{l}\text { Sig. } \\
(p)\end{array}$ \\
\hline Non-modification & $\begin{array}{l}37.22 \% \\
(166)\end{array}$ & $\begin{array}{l}50.95 \% \\
(403)\end{array}$ & $\begin{array}{l}46.00 \% \\
(569)\end{array}$ & & \\
\hline \multicolumn{6}{|l|}{ Modification } \\
\hline - Switching & $\begin{array}{l}9.19 \% \\
(41)\end{array}$ & $\begin{array}{l}6.32 \% \\
(50)\end{array}$ & $\begin{array}{l}7.36 \% \\
(91)\end{array}$ & $\chi^{2}=22.95$ & $0.00 *$ \\
\hline - Augmentation & $\begin{array}{l}14.13 \% \\
(63)\end{array}$ & $\begin{array}{l}9.86 \% \\
(78)\end{array}$ & $\begin{array}{l}11.40 \% \\
(141)\end{array}$ & & \\
\hline Discontinuation & $\begin{array}{l}39.46 \% \\
(176)\end{array}$ & $\begin{array}{l}32.87 \% \\
(260)\end{array}$ & $\begin{array}{l}35.25 \% \\
(436)\end{array}$ & & \\
\hline
\end{tabular}

*significance at the 0.05 level $\chi^{2}=$ Chi-square test 
Table 6A: Multinomial Logistic regression for the impact of depression on pattern of oral hypoglycemic use (Switching vs. Non-modification)

\begin{tabular}{lcccccc}
\hline & Beta & S.E. & $\begin{array}{c}\text { Sig. } \\
\text { pig) }\end{array}$ & $\begin{array}{c}\text { Odds } \\
\text { ratio }\end{array}$ & $\begin{array}{c}\text { 95\% C.I. for } \\
\text { Odds ratio } \\
\text { Lower Upper }\end{array}$ \\
\hline Depression & & & & & & Upper
\end{tabular}

Demographic characteristics

\begin{tabular}{|c|c|c|c|c|c|c|}
\hline Age (in years) & 0.006 & 0.013 & 0.674 & 1.006 & 0.980 & 1.032 \\
\hline Males (ref: females) & 0.223 & 0.260 & 0.391 & 1.249 & 0.751 & 2.078 \\
\hline Whites (ref: non-whites) & 1.156 & 0.777 & 0.137 & 3.178 & 0.692 & 14.588 \\
\hline Urban (ref: rural) & 0.180 & 0.462 & 0.697 & 1.197 & 0.484 & 2.957 \\
\hline \multicolumn{7}{|c|}{ Year of index prescription (ref: 2001) } \\
\hline - 1998 & -0.911 & 0.634 & 0.151 & 0.402 & 0.116 & 1.394 \\
\hline - 1999 & -0.614 & 0.589 & 0.297 & 0.541 & 0.171 & 716 \\
\hline - 2000 & -0.261 & 0.559 & 0.640 & 0.770 & 0.258 & \\
\hline
\end{tabular}

Co-morbid conditions in the pre period

Number of ER/hosp visits $\quad 0.051$

$\begin{array}{lllll}0.064 & 0.428 & 1.052 & 0.928 & 1.193\end{array}$

$\begin{array}{lllllll}\text { Total health costs } & 0.000 & 0.000 & 0.256 & 1.000 & 1.000 & 1.000\end{array}$

$\begin{array}{lllllll}\text { Charlson co-morbidity } & -0.051 & 0.144 & 0.722 & 0.950 & 0.716 & 1.260\end{array}$

$\begin{array}{lllllll}\text { Number of therapeutic classes } & 0.077 & 0.131 & 0.556 & 1.080 & 0.835 & 1.397\end{array}$

of medications

$\begin{array}{lllllll}\text { Cardio-vascular } & -0.395 & 0.274 & 0.149 & 0.674 & 0.394 & 1.152\end{array}$

$\begin{array}{lllllll}\text { Cancer } & 0.446 & 0.630 & 0.479 & 1.563 & 0.455 & 5.372\end{array}$

$\begin{array}{lllllll}\text { Asthma } & 0.290 & 0.358 & 0.418 & 1.336 & 0.662 & 2.696\end{array}$

$\begin{array}{lllllll}\text { Ulcers } & 0.728 & 0.601 & 0.225 & 2.072 & 0.638 & 6.724\end{array}$

Provider interaction in the pre period

$\begin{array}{lllllll}\text { PAAS enrollment } & 0.413 & 0.347 & 0.234 & 1.512 & 0.765 & 2.986\end{array}$

Number of pharmacies visited (ref: multiple pharmacies)

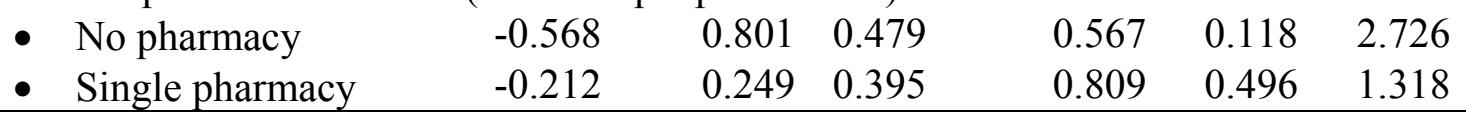


Table 6A: Multinomial Logistic regression for the impact of depression on pattern of oral hypoglycemic use (Switching vs. Non-modification) (Contd.)

\begin{tabular}{|c|c|c|c|c|c|c|}
\hline & \multirow[t]{2}{*}{ Beta } & \multirow[t]{2}{*}{ S.E. } & \multirow[t]{2}{*}{$\begin{aligned} \text { Sig. } \\
\text { (p) }\end{aligned}$} & \multirow[t]{2}{*}{$\begin{array}{l}\text { Odds } \\
\text { ratio }\end{array}$} & \multicolumn{2}{|c|}{$\begin{array}{r}95 \% \text { C.I. for } \\
\text { Odds ratio }\end{array}$} \\
\hline & & & & & Lower & Upper \\
\hline $\begin{array}{l}\text { Complexity of regimen } \\
\text { Number of pills per day for } \\
\text { chronic medications }\end{array}$ & -0.035 & 0.059 & 0.557 & 0.966 & 0.859 & 1.085 \\
\hline $\begin{array}{l}\text { Number of prescriptions for } \\
\text { chronic medications }\end{array}$ & -0.005 & 0.024 & 0.849 & 0.995 & 0.949 & 1.044 \\
\hline $\begin{array}{l}\text { Number of pills per day for } \\
\text { oral hypoglycemics }\end{array}$ & 0.122 & 0.217 & 0.575 & 1.129 & 0.738 & 1.728 \\
\hline $\begin{array}{l}\text { Diabetes severity } \\
\text { Index oral hypoglycel }\end{array}$ & iption & f: Fami & ly me & & & \\
\hline $\begin{array}{l}\text { - Endocrinologist } \\
\text { - Internal medicine }\end{array}$ & $\begin{array}{l}1.355 \\
0.335\end{array}$ & $\begin{array}{l}0.885 \\
0.255\end{array}$ & $\begin{array}{l}0.126 \\
0.189\end{array}$ & $\begin{array}{l}3.876 \\
1.398\end{array}$ & $\begin{array}{l}0.684 \\
0.848\end{array}$ & $\begin{array}{r}21.956 \\
2.305\end{array}$ \\
\hline Total diabetes costs & 0.000 & 0.000 & 0.720 & 1.000 & 1.000 & 1.000 \\
\hline $\begin{array}{l}\text { Number of diabetes related } \\
\text { ER/Hosp visits }\end{array}$ & 0.311 & 0.305 & 0.309 & 1.364 & 0.750 & 2.481 \\
\hline $\begin{array}{l}\text { Gap between index diagnosis } \\
\text { and prescription }\end{array}$ & -0.001 & 0.001 & 0.167 & 0.999 & 0.998 & 1.000 \\
\hline \multicolumn{7}{|c|}{ Index class of oral hypoglycemic (ref: Sulfonyl urea) } \\
\hline - Thiazolidenedione & 0.327 & 0.447 & 0.465 & 1.386 & 0.577 & 3.331 \\
\hline - Biguanides & -0.324 & 0.330 & 0.327 & 0.724 & 0.379 & 1.383 \\
\hline - Alpha-glucosidase & 1.166 & 0.739 & 0.115 & 3.208 & 0.753 & 13.658 \\
\hline - Meglitinides & 0.377 & 0.808 & 0.641 & 1.457 & 0.299 & 7.107 \\
\hline Constant & -3.118 & 1.302 & 0.017 & & & \\
\hline
\end{tabular}


Table 6B: Multinomial Logistic regression for the impact of depression on pattern of oral hypoglycemic use (Augmentation vs. Non-modification)

\begin{tabular}{lcccccc}
\hline Beta & S.E. & $\begin{array}{l}\text { Sig. } \\
\text { p }\end{array}$ & $\begin{array}{c}\text { Odds } \\
\text { ratio }\end{array}$ & $\begin{array}{c}\text { 95\% C.I. for } \\
\text { Odds ratio } \\
\text { Lower }\end{array}$ \\
\hline Depression & & & & & & Uppr
\end{tabular}

\section{Demographic characteristics}

\begin{tabular}{|c|c|c|c|c|c|c|}
\hline Age (in years) & -0.008 & 0.011 & 0.452 & 0.992 & 0.972 & 1.013 \\
\hline Males (ref: females) & -0.018 & 0.218 & 0.933 & 0.982 & 0.640 & 1.507 \\
\hline Whites (ref: non-whites) & 0.744 & 0.552 & 0.178 & 2.104 & 0.713 & 6.207 \\
\hline Urban (ref: rural) & 0.436 & 0.406 & 0.283 & 1.547 & 0.698 & 3.429 \\
\hline \multicolumn{7}{|c|}{ Year of index prescription (ref: 2001) } \\
\hline • 1998 & -0.328 & 0.559 & 0.558 & 0.720 & 0.241 & 2.156 \\
\hline - 1999 & -0.007 & 0.523 & 0.989 & 0.993 & 0.356 & 2.767 \\
\hline - 2000 & 0.335 & 0.502 & 0.505 & 1.398 & 0.523 & 3.738 \\
\hline \multicolumn{7}{|c|}{ Co-morbid conditions in the pre period } \\
\hline Number of ER/hosp visits & 0.141 & 0.047 & $0.003 *$ & 1.151 & 1.051 & 1.262 \\
\hline Total health costs & 0.000 & 0.000 & 0.052 & 1.000 & 1.000 & 1.000 \\
\hline Charlson co-morbidity & -0.248 & 0.154 & 0.108 & 0.781 & 0.577 & 1.056 \\
\hline $\begin{array}{l}\text { Number of therapeutic classes } \\
\text { of medications }\end{array}$ & 0.201 & 0.109 & 0.065 & 1.222 & 0.988 & 1.512 \\
\hline Cardio-vascular & -0.152 & 0.226 & 0.503 & 0.859 & 0.551 & 1.339 \\
\hline Cancer & 0.143 & 0.617 & 0.817 & 1.153 & 0.344 & 3.867 \\
\hline Asthma & 0.049 & 0.336 & 0.885 & 1.050 & 0.543 & 2.029 \\
\hline Ulcers & 0.367 & 0.635 & 0.564 & 1.443 & 0.416 & 5.012 \\
\hline \multicolumn{7}{|c|}{ Provider interaction in the pre period } \\
\hline PAAS enrollment & 0.325 & 0.300 & 0.279 & 1.384 & 0.769 & 2.489 \\
\hline \multicolumn{7}{|c|}{ Number of pharmacies visited (ref: multiple pharmacies) } \\
\hline - No pharmacy & 0.650 & 0.532 & 0.222 & 1.915 & 0.675 & 5.429 \\
\hline - Single pharmacy & 0.060 & 0.208 & 0.773 & 1.062 & 0.706 & 1.598 \\
\hline
\end{tabular}


Table 6B: Multinomial Logistic regression for the impact of depression on pattern of oral hypoglycemic use (Augmentation vs. Non-modification) (Contd.)

\begin{tabular}{|c|c|c|c|c|c|c|}
\hline & \multirow[t]{2}{*}{ Beta } & \multirow[t]{2}{*}{ S.E. } & \multirow[t]{2}{*}{$\begin{array}{l}\text { Sig. } \\
\text { (p) }\end{array}$} & \multirow[t]{2}{*}{$\begin{array}{l}\text { Odds } \\
\text { ratio }\end{array}$} & \multicolumn{2}{|c|}{$\begin{array}{r}95 \% \text { C.I. for } \\
\text { Odds ratio }\end{array}$} \\
\hline & & & & & Lower & Upper \\
\hline \multicolumn{7}{|l|}{ Complexity of regimen } \\
\hline $\begin{array}{l}\text { Number of pills per day for } \\
\text { chronic medications }\end{array}$ & -0.018 & 0.048 & 0.705 & 0.982 & 0.895 & 1.078 \\
\hline $\begin{array}{l}\text { Number of prescriptions for } \\
\text { chronic medications }\end{array}$ & -0.014 & 0.020 & 0.486 & 0.986 & 0.947 & 1.026 \\
\hline $\begin{array}{l}\text { Number of pills per day for } \\
\text { oral hypoglycemics }\end{array}$ & 0.307 & 0.168 & 0.067 & 1.359 & 0.978 & 1.887 \\
\hline \multicolumn{7}{|l|}{ Diabetes severity } \\
\hline \multicolumn{7}{|c|}{ Index oral hypoglycemic prescription by: (ref: Family medicine) } \\
\hline - Endocrinologist & 1.469 & 0.698 & $0.035 *$ & 4.347 & 1.107 & 17.072 \\
\hline - Internal medicine & 0.152 & 0.217 & 0.484 & 1.164 & 0.761 & 1.781 \\
\hline Total diabetes costs & 0.000 & 0.000 & $0.032 *$ & 1.000 & 1.000 & 1.000 \\
\hline $\begin{array}{l}\text { Number of diabetes related } \\
\text { ER/Hosp visits }\end{array}$ & -0.348 & 0.291 & 0.232 & 0.706 & 0.399 & 1.250 \\
\hline $\begin{array}{l}\text { Gap between index diagnosis } \\
\text { and prescription }\end{array}$ & 0.000 & 0.001 & 0.508 & 1.000 & 0.998 & 1.001 \\
\hline \multicolumn{7}{|c|}{ Index class of oral hypoglycemic (ref: Sulfonyl urea) } \\
\hline - Thiazolidenedione & 0.133 & 0.419 & 0.750 & 1.143 & 0.503 & 2.598 \\
\hline - Biguanides & -0.089 & 0.257 & 0.730 & 0.915 & 0.553 & 1.515 \\
\hline - Alpha-glucosidase & -0.505 & 0.888 & 0.569 & 0.603 & 0.106 & 3.442 \\
\hline - Meglitinides & -1.095 & 0.926 & 0.237 & 0.335 & 0.054 & 2.056 \\
\hline Constant & -3.016 & 1.048 & 0.004 & & & \\
\hline
\end{tabular}


Table 6C: Multinomial Logistic regression for the impact of depression on pattern of oral hypoglycemic use (Discontinuation vs. Non-modification)

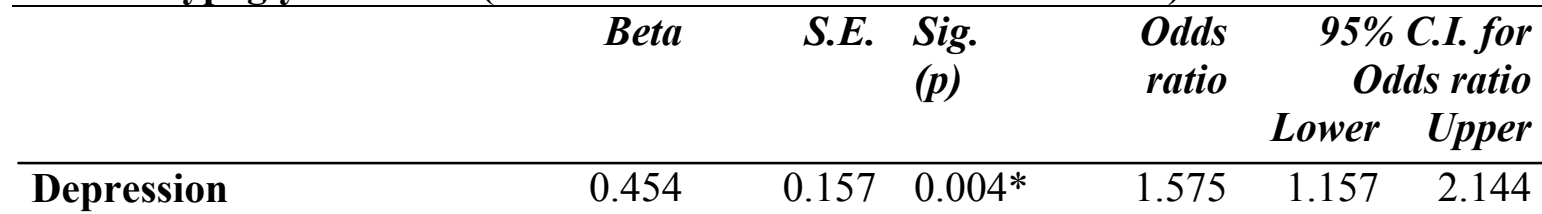

Demographic characteristics

\begin{tabular}{|c|c|c|c|c|c|c|}
\hline Age (in years) & -0.013 & 0.007 & 0.065 & 0.987 & 0.973 & 1.001 \\
\hline Males (ref: females) & -0.053 & 0.150 & 0.723 & 0.948 & 0.706 & 1.273 \\
\hline Whites (ref: non-whites) & -0.056 & 0.295 & 0.850 & 0.946 & 0.531 & 1.685 \\
\hline Urban (ref: rural) & 0.077 & 0.259 & 0.767 & 1.080 & 0.650 & 1.795 \\
\hline \multicolumn{7}{|c|}{ Year of index prescription (ref: 2001) } \\
\hline - 1998 & -0.782 & 0.385 & $0.042 *$ & 0.458 & 0.215 & 0.973 \\
\hline - 1999 & -0.859 & 0.366 & $0.019 *$ & 0.424 & 0.207 & 0.869 \\
\hline - 2000 & -0.518 & 0.351 & 0.140 & 0.596 & 0.299 & 1.186 \\
\hline \multicolumn{7}{|c|}{ Co-morbid conditions in the pre period } \\
\hline Number of ER/hosp visits & 0.148 & 0.037 & $0.000 *$ & 1.159 & 1.079 & 1.246 \\
\hline Total health costs & 0.000 & 0.000 & 0.218 & 1.000 & 1.000 & 1.000 \\
\hline Charlson co-morbidity & 0.077 & 0.082 & 0.343 & 1.080 & 0.921 & 1.268 \\
\hline $\begin{array}{l}\text { Number of therapeutic classes } \\
\text { of medications }\end{array}$ & 0.083 & 0.080 & 0.300 & 1.087 & 0.929 & 1.271 \\
\hline Cardio-vascular & -0.311 & 0.156 & $0.046 *$ & 0.732 & 0.539 & 0.995 \\
\hline Cancer & 0.429 & 0.392 & 0.273 & 1.536 & 0.713 & 3.310 \\
\hline Asthma & 0.290 & 0.215 & 0.178 & 1.337 & 0.877 & 2.038 \\
\hline Ulcers & 0.051 & 0.415 & 0.903 & 1.052 & 0.467 & 2.370 \\
\hline
\end{tabular}

Provider interaction in the pre period

$\begin{array}{lllllll}\text { PAAS enrollment } & 0.043 & 0.216 & 0.843 & 1.044 & 0.683 & 1.595\end{array}$

Number of pharmacies visited (ref: multiple pharmacies)

\begin{tabular}{|c|c|c|c|c|c|c|}
\hline $\begin{array}{l}\text { - No pharmacy } \\
\text { - Single pharmacy }\end{array}$ & $\begin{array}{r}0.377 \\
-0.186\end{array}$ & $\begin{array}{l}0.340 \\
0.145\end{array}$ & $\begin{array}{l}0.267 \\
0.201\end{array}$ & $\begin{array}{l}1.458 \\
0.830\end{array}$ & $\begin{array}{l}0.749 \\
0.624\end{array}$ & $\begin{array}{l}2.838 \\
1.104\end{array}$ \\
\hline
\end{tabular}


Table 6C: Multinomial Logistic regression for the impact of depression on pattern of oral hypoglycemic use (Discontinuation vs. Non-modification) (Contd.)

\begin{tabular}{|c|c|c|c|c|c|c|}
\hline & \multirow[t]{2}{*}{ Beta } & \multirow[t]{2}{*}{ S.E. } & \multirow[t]{2}{*}{$\begin{array}{l}\text { Sig. } \\
(p)\end{array}$} & \multirow[t]{2}{*}{$\begin{array}{l}\text { Odds } \\
\text { ratio }\end{array}$} & \multicolumn{2}{|c|}{$\begin{array}{r}95 \% \text { C.I. for } \\
\text { Odds ratio }\end{array}$} \\
\hline & & & & & Lower & Upper \\
\hline $\begin{array}{l}\text { Complexity of regimen } \\
\text { Number of pills per day for } \\
\text { chronic medications }\end{array}$ & 0.032 & 0.033 & 0.327 & 1.033 & 0.968 & 1.102 \\
\hline $\begin{array}{l}\text { Number of prescriptions for } \\
\text { chronic medications }\end{array}$ & -0.060 & 0.016 & $0.000 *$ & 0.942 & 0.913 & 0.971 \\
\hline $\begin{array}{l}\text { Number of pills per day for } \\
\text { oral hypoglycemics }\end{array}$ & 0.103 & 0.126 & 0.414 & 1.109 & 0.866 & 1.421 \\
\hline \multicolumn{7}{|l|}{ Diabetes severity } \\
\hline $\begin{array}{l}\text { - Endocrinologist } \\
\text { - Internal medicine }\end{array}$ & $\begin{array}{l}0.312 \\
0.171\end{array}$ & $\begin{array}{l}0.694 \\
0.151\end{array}$ & $\begin{array}{l}0.653 \\
0.259\end{array}$ & $\begin{array}{l}1.366 \\
1.187\end{array}$ & $\begin{array}{l}0.350 \\
0.882\end{array}$ & $\begin{array}{l}5.321 \\
1.597\end{array}$ \\
\hline Total diabetes costs & 0.000 & 0.000 & 0.639 & 1.000 & 1.000 & 1.000 \\
\hline $\begin{array}{l}\text { Number of diabetes related } \\
\text { ER/Hosp visits }\end{array}$ & -0.296 & 0.222 & 0.182 & 0.744 & 0.481 & 1.149 \\
\hline $\begin{array}{l}\text { Gap between index diagnosis } \\
\text { and prescription }\end{array}$ & -0.001 & 0.000 & $0.027 *$ & 0.999 & 0.998 & 1.000 \\
\hline \multicolumn{7}{|c|}{ Index class of oral hypoglycemic (ref: Sulfonyl urea) } \\
\hline - Thiazolidenedione & 0.094 & 0.300 & 0.753 & 1.099 & 0.610 & 1.980 \\
\hline - Biguanides & 0.127 & 0.183 & 0.487 & 1.136 & 0.793 & 1.627 \\
\hline - Alpha-glucosidase & -0.366 & 0.633 & 0.564 & 0.694 & 0.201 & 2.400 \\
\hline - Meglitinides & -0.254 & 0.586 & 0.664 & 0.776 & 0.246 & 2.444 \\
\hline Constant & 1.042 & 0.667 & 0.118 & & & \\
\hline
\end{tabular}

*significance at the 0.05 level

PAAS = Physician Assured Access System

\section{Model fit statistics:}

Pseudo R-square $=16.1 \%$

-2 Log Likelihood $=2616.299 ; \chi^{2}=189.814 ; p=0.00$ 


\section{Discussion for Objective 3}

Depression can play an important role in diabetes management as it can decrease adherence with medications leading to inadequate glucose control and thus affect patterns of use of oral hypoglycemics. Results from our study indicated the same with a significantly higher fraction of depressed patients switching, augmenting, and discontinuing therapy. There are no studies in literature to compare these results as no other study has examined the impact of depression on patterns of oral hypoglycemic use. However, there are a few studies, which examine the patterns of oral hypoglycemic use in newly diagnosed type 2 diabetes patients.

A large administrative database study by Boccuzzi and associates ${ }^{16}$ using pharmacy claims of millions of Americans, found that the rate of discontinuation for newly diagnosed type 2 diabetes patients in the 12 -month follow up period was $11.3 \%$. Nearly $15 \%$ augmented therapy while $10 \%$ switched from the index oral hypoglycemic agent in the 12-month follow up period. A similar study conducted by Skaer and associates ${ }^{14}$ estimated that $10-30 \%$ of patients with type 2 diabetes withdraw from their prescribed regimen within 1 year of diagnosis. In a study conducted by Dailey and associates $^{90}$ in Medi-Cal patients who were newly diagnosed with type 2 diabetes, patterns of oral hypoglycemic use indicated that nearly $16 \%$ of patients failed to refill their index oral hypoglycemic prescription in the one-year follow up period. Donnan and associates ${ }^{17}$ conducted a population-based study in Tayside, Scotland following up newly diagnosed type 2 diabetes patients for varying time periods with a median follow up time of 588 days. Results from the study indicated that nearly $70.00 \%$ of patients did not modify their original oral hypoglycemic therapy, $5.40 \%$ augmented therapy, and around 
$18.00 \%$ switched therapy in the follow up time period. Surprisingly, only $0.50 \%$ of the newly diagnosed type 2 diabetes patients discontinued pharmacotherapy.

The overall rates of switching (7.40\%) and augmentation (11.40\%) found in this study are comparable to the findings in literature. The results showed lower rates of nonmodification with $46.00 \%$ of newly diagnosed patients maintaining therapy on the index oral hypoglycemic agent, as compared to non-modification rates of $60-70 \%$ in the literature. The largest discrepancy, however, was in terms of discontinuation rates. The rates of $35.00 \%$ were much higher than most reported studies. The differences in these patterns can be attributed to differences in algorithm used for classification of these patterns and/or variations in length of follow-up periods. These differences can also be attributable to a difference in the health care system, as some of the studies in literature were based in Europe. Also, a number of these studies had the strict inclusion criteria of at least two prescriptions for oral hypoglycemics in the follow up as opposed to our criteria of a diagnosis of type 2 diabetes and at least one prescription for an oral hypoglycemic agent. This difference in inclusion criteria can possibly be the most logical reason for higher discontinuation rates in this study. However, similar studies conducted to examine patterns of use for antihypertensive medications have detected high discontinuation rates comparable to this study results with $40-50 \%$ of new users of antihypertensive medications discontinuing use by 6 months. ${ }^{160}$

Patterns of use of oral hypoglycemics have been used in the literature to examine management of patients with newly diagnosed type 2 diabetes. They can be highly indicative of success of oral hypoglycemic therapy in controlling blood glucose levels in these patients. Non-modification of the initially prescribed oral hypoglycemic therapy 
can be an indication of adequate blood glucose control. Modification of therapy in terms of either switching or augmenting therapies is typically seen when the index oral hypoglycemic therapy fails to maintain blood glucose levels. This inadequate control of blood glucose can occur due to the natural course of the disease over time. However, frequently it is a result of lack of adherence to either medications, diet or exercise regimen. The act of switching/augmentation typically indicates secondary failure with initial therapy. However, such a conclusion cannot be drawn for patients discontinuing pharmacotherapy. The reasons for discontinuing therapy are not captured in a claims database. The adverse effects of oral hypoglycemic agents can affect patient's quality of life, which in turn can result in discontinuation of treatment. Thus, these patients may be discontinuing therapy in spite of inadequate blood glucose control, which may cause a number of diabetic complications in the future. It is also possible that patients discontinue pharmacotherapy as their blood glucose levels are controlled through other methods such as diet and exercise. However, a review of 13,309 patients with type 2 diabetes from the UK Mediphys database, demonstrated that patients who discontinued their oral hypoglycemics were approximately twice as likely to experience an emergency medical event and had a mortality rate that was three times that of continuers. ${ }^{13}$ These results indicate that a large fraction of type 2 diabetes patients who discontinue therapy need to maintain pharmacotherapy for adequate management of their diabetes.

Although no studies are available in the literature to examine the impact of depression on patterns of oral hypoglycemic use, studies have demonstrated an association between depression and adherence to diabetes medications. ${ }^{28}$ Patterns such as switching, augmentation, and especially discontinuation can be indicative of lack of 
adherence. Results of this study demonstrated that patterns of oral hypoglycemic use were much more favorable in non-depressed patients as compared to depressed patients after adjusting for the effect of confounders. This study demonstrates that even in a population with complete insurance coverage, there may be issues of avoidance of therapy partly due to presence of co-morbid depression.

\section{Results for Objective 4}

To examine the impact of pre-existing depression on secondary failure with oral hypoglycemics

Augmenting or switching from the initial class of oral hypoglycemic agent can be considered together as modification of therapy, indicative of secondary failure with initial pharmacotherapy. Objective 4 was to estimate the impact of depression on the rate of modification and the time to modification with index therapy in the 12-month follow up period. Patients initiated on polytherapy or insulin therapy were excluded from the analysis. Patients who discontinued therapy in the 12-month follow up period were also excluded from the analysis.

Univariate results are presented in Table 7 . The results indicated that a significantly higher percent of depressed patients (38.47\%) modified their index oral hypoglycemic therapy as compared to non-depressed patients $(24.08 \%)$. Higher rates of modification were also accompanied with a relatively shorter time period to modification for depressed patients $(282.57 \pm 7.54)$ compared to non-depressed patients $(307.63 \pm$ 4.99). A univariate Kaplan Meier analysis indicated that this time to modification was significantly higher for depressed patients with a log rank test statistic of 16.65 
$(\mathrm{p}=0.000)$. These results can also be seen in terms of a better survival distribution for non-depressed patients in the Kaplan Meier survival curves for time to modification of pharmacotherapy (Figure 6). However, this effect could also be a result of confounders such as demographics, co-morbidity, diabetes severity, complexity of regimen, and interaction with health care providers. Hence, a multivariate logistic regression and Cox proportional hazards model was performed to determine the impact of depression on rate and time to modification of oral hypoglycemic use controlling for baseline confounding factors. The logistic regression model was found to be significant with a Nagelkerke Rsquare value of $11.40 \%$. The model results are summarized in Table 8 . Results of the multivariate model indicated that controlling for confounding covariates, patients with depression were nearly 1.7 more likely to modify oral hypoglycemic therapy in the 12 month follow up period as compared to patients without depression $(\mathrm{p}=0.003)$. An initial multivariate Cox proportional hazards model to estimate the impact of depression on time to modification of index oral hypoglycemic therapy failed to satisfy the proportional hazard assumption. Hence, on the basis of the cumulative Kaplan Meier survival functions that indicated 2 different hazards, an extended Cox-proportional hazards model was estimated to examine the effect of depression on time to modification in the first six months and the latter six month follow up period. The extended Cox proportional hazards model was found to be significant and the model fit and results are summarized in Table 9. The model indicated that the hazard to modify therapy was 2.4 times more for depressed patients as compared to non-depressed patients in the latter six months of the follow-up period $(\mathrm{p}=0.001)$. No significant differences were observed 
between the patients for the first six months after the index oral hypoglycemic prescription. 
Table 7: Modification of oral hypoglycemics in the 12-month follow up period

\begin{tabular}{|c|c|c|c|c|c|}
\hline & $\begin{array}{c}\text { Depressed } \\
(n=270)\end{array}$ & $\begin{array}{c}\text { Non-depressed } \\
(n=531)\end{array}$ & $\begin{array}{c}\text { Total } \\
(n=801)\end{array}$ & $\begin{array}{c}\text { Test } \\
\text { statistic }\end{array}$ & $\begin{array}{l}\text { Sig. } \\
(p)\end{array}$ \\
\hline Non-modification & $\begin{array}{l}61.53 \% \\
(166)\end{array}$ & $\begin{array}{l}75.92 \% \\
(403)\end{array}$ & $\begin{array}{l}71.02 \% \\
(569)\end{array}$ & & \\
\hline Modification & $\begin{array}{l}38.47 \% \\
(104)\end{array}$ & $\begin{array}{l}24.08 \% \\
(128)\end{array}$ & $\begin{array}{l}28.98 \% \\
(232)\end{array}$ & $\chi^{2}=18.07$ & $0.000^{*}$ \\
\hline $\begin{array}{l}\text { Time to modification } \\
\text { (in years) } \\
\text { (Mean + Standard Error) }\end{array}$ & $\begin{array}{r}282.57 \\
( \pm 7.54)\end{array}$ & $\begin{array}{r}307.63 \\
( \pm 4.99)\end{array}$ & $\begin{array}{l}299.18 \\
( \pm 4.19)\end{array}$ & $\mathrm{LR}=16.65$ & $0.000 *$ \\
\hline
\end{tabular}


Figure 6: Kaplan-Meier Survival Curves for time to modification of oral hypoglycemic therapy

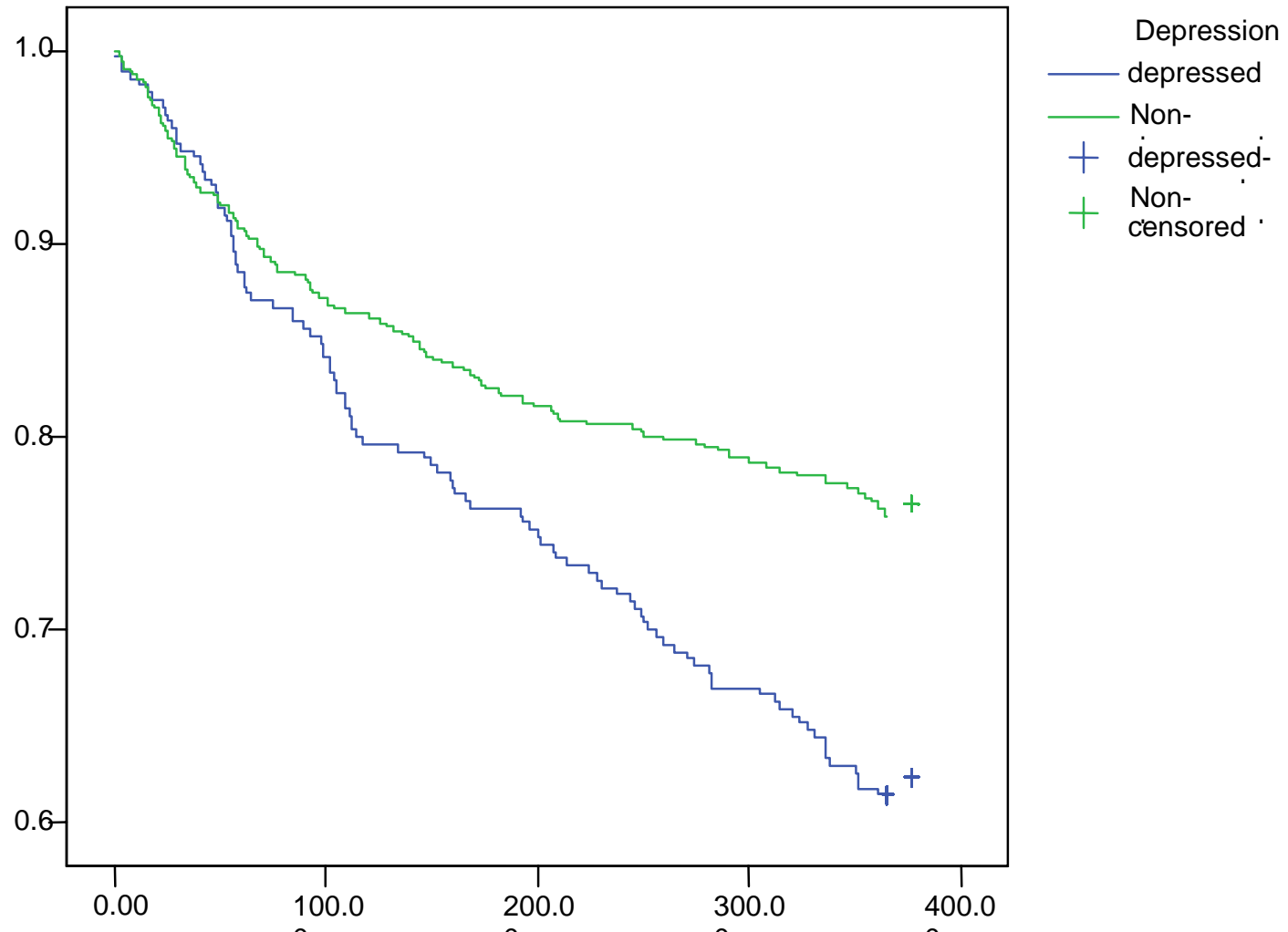

Time to modification (switching, augmentation) in the 12- month follow up 
Table 8: Logistic regression for the impact of depression on modification of oral hypoglycemic agents

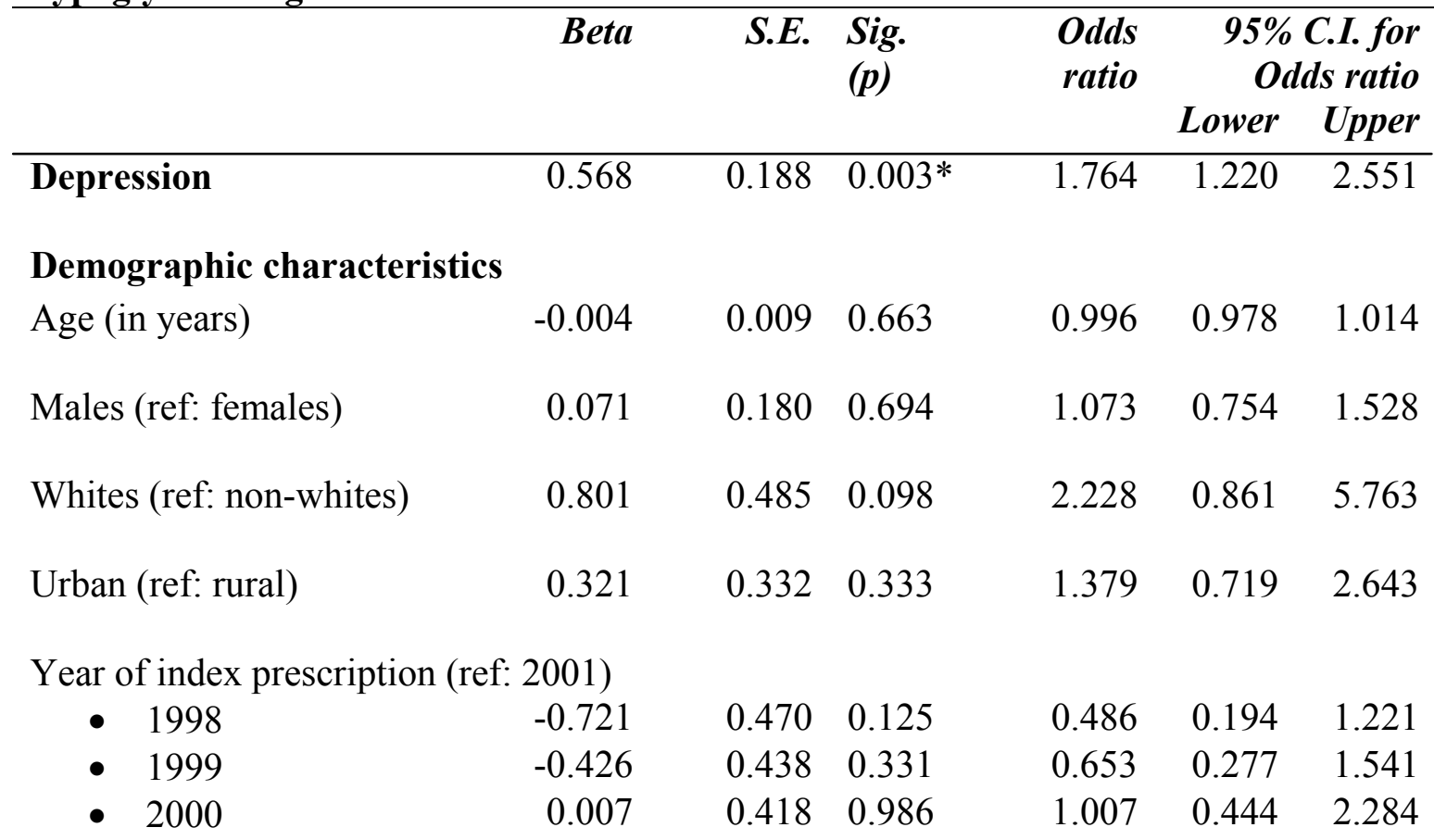

Co-morbid conditions in the pre period

Number of ER/hosp visits

$\begin{array}{lllll}0.045 & 0.015^{*} & 1.116 & 1.022 & 1.219\end{array}$

Total health costs

$\begin{array}{llllll}0.000 & 0.000 & 0.028^{*} & 1.000 & 1.000 & 1.000\end{array}$

Charlson co-morbidity

$-0.174$

$0.119 \quad 0.145$

$\begin{array}{lll}0.840 & 0.665 \quad 1.062\end{array}$

Number of therapeutic classes

0.174

$0.095 \quad 0.068$

$\begin{array}{lll}1.190 & 0.987 & 1.434\end{array}$

of medications

Cardio-vascular

$\begin{array}{lll}-0.257 & 0.196 \quad 0.190\end{array}$

$0.773 \quad 0.527$

1.135

Cancer

0.295

$0.512 \quad 0.564$

1.344

$0.493 \quad 3.665$

Asthma

$\begin{array}{lll}0.125 & 0.273 & 0.647\end{array}$

$1.133 \quad 0.663$

1.937

Ulcers

$\begin{array}{lll}0.594 & 0.494 & 0.229\end{array}$

$\begin{array}{lll}1.810 & 0.688 \quad 4.764\end{array}$

Provider interaction in the pre period

PAAS enrollment

0.420

$0.253 \quad 0.097$

$\begin{array}{lll}1.522 & 0.927 & 2.499\end{array}$

Number of pharmacies visited (ref: multiple pharmacies)

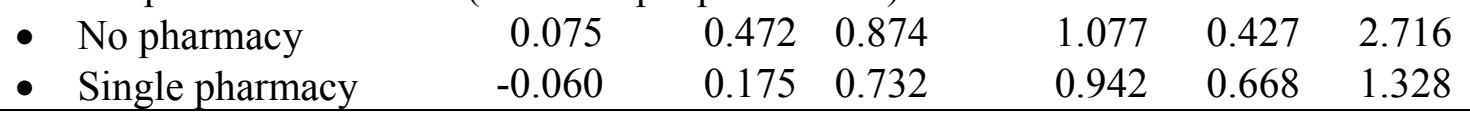


Table 8: Logistic regression for the impact of depression on modification of oral hypoglvcemic agents (Contd.)

\begin{tabular}{|c|c|c|c|c|c|c|}
\hline & \multirow[t]{2}{*}{ Beta } & \multirow[t]{2}{*}{ S.E. } & \multirow[t]{2}{*}{$\begin{array}{l}\text { Sig. } \\
\text { (p) }\end{array}$} & \multirow[t]{2}{*}{$\begin{array}{l}\text { Odds } \\
\text { ratio }\end{array}$} & \multicolumn{2}{|c|}{$\begin{array}{r}\text { 95\% C.I. for } \\
\text { Odds ratio }\end{array}$} \\
\hline & & & & & Lower & Upper \\
\hline $\begin{array}{l}\text { Complexity of regimen } \\
\text { Number of pills per day for } \\
\text { chronic medications }\end{array}$ & -0.030 & 0.040 & 0.465 & 0.971 & 0.897 & 1.051 \\
\hline $\begin{array}{l}\text { Number of prescriptions for } \\
\text { chronic medications }\end{array}$ & -0.012 & 0.018 & 0.514 & 0.988 & 0.954 & 1.024 \\
\hline $\begin{array}{l}\text { Number of pills per day for } \\
\text { oral hypoglycemics }\end{array}$ & 0.292 & 0.153 & 0.056 & 1.340 & 0.993 & 1.808 \\
\hline $\begin{array}{l}\text { Diabetes severity } \\
\text { Index oral hypoglycemic presc }\end{array}$ & iption $b$ & ef: Fam & ly medic & & & \\
\hline $\begin{array}{l}\text { - Endocrinologist } \\
\text { - Internal medicine }\end{array}$ & $\begin{array}{l}1.533 \\
0.212\end{array}$ & $\begin{array}{l}0.647 \\
0.183\end{array}$ & $\begin{array}{l}0.018 * \\
0.245\end{array}$ & $\begin{array}{l}4.633 \\
1.237\end{array}$ & $\begin{array}{l}1.303 \\
0.865\end{array}$ & $\begin{array}{r}16.473 \\
1.769\end{array}$ \\
\hline Total diabetes costs & 0.000 & 0.000 & 0.200 & 1.000 & 1.000 & 1.000 \\
\hline $\begin{array}{l}\text { Number of diabetes related } \\
\text { ER/Hosp visits }\end{array}$ & -0.030 & 0.232 & 0.896 & 0.970 & 0.615 & 1.530 \\
\hline $\begin{array}{l}\text { Gap between index diagnosis } \\
\text { and prescription }\end{array}$ & -0.001 & 0.001 & 0.187 & 0.999 & 0.998 & 1.000 \\
\hline Index class of oral hypoglycem & c (ref: $s$ & nyl ure & & & & \\
\hline - Thiazolidenedione & 0.181 & 0.344 & 0.598 & 1.199 & 0.611 & 2.351 \\
\hline - Biguanides & -0.234 & 0.227 & 0.302 & 0.791 & 0.507 & 1.234 \\
\hline - Alpha-glucosidase & 0.348 & 0.663 & 0.600 & 1.416 & 0.386 & 5.191 \\
\hline - Meglitinides & -0.261 & 0.664 & 0.694 & 0.770 & 0.210 & 2.829 \\
\hline Constant & -2.093 & 0.893 & 0.019 & 0.123 & & \\
\hline
\end{tabular}

*significance at the 0.05 level

PAAS = Physician Assured Access System

Model fit statistics:

Pseudo R-square $=11.4 \%$

-2 Log Likelihood $=871.997 ; \chi^{2}=64.944, \mathrm{p}=0.000$

Hosmer and Lemeshow's goodness of fit: $\chi^{2}=8.157 ; \mathrm{p}=0.418$ 
Table 9: Extended Cox proportional hazards model for the impact of depression on time to modification of oral hypoglycemic agent

\begin{tabular}{|c|c|c|c|c|c|c|}
\hline & \multirow[t]{2}{*}{ Beta } & \multirow[t]{2}{*}{ S.E. } & \multirow[t]{2}{*}{$\begin{array}{l}\text { Sig. } \\
(p)\end{array}$} & \multirow[t]{2}{*}{$\begin{array}{r}\text { Hazard } \\
\text { ratio }\end{array}$} & \multicolumn{2}{|c|}{$\begin{array}{l}\text { 95\% C.I. for } \\
\text { Hazard ratio }\end{array}$} \\
\hline & & & & & Lower & Upper \\
\hline Depression (0 -6 months) & 0.168 & 0.181 & 0.353 & 1.183 & 0.829 & 1.688 \\
\hline Depression (6 -12 months) & 0.855 & 0.246 & $0.001 *$ & 2.350 & 1.451 & 3.806 \\
\hline \multicolumn{7}{|l|}{ Demographic characteristics } \\
\hline Age (in years) & -0.004 & 0.008 & 0.623 & 0.996 & 0.982 & 1.011 \\
\hline Males (ref: females) & 0.028 & 0.150 & 0.852 & 1.028 & 0.767 & 1.379 \\
\hline Whites (ref: non-whites) & 0.749 & 0.434 & 0.084 & 2.115 & 0.904 & 4.948 \\
\hline Urban (ref: rural) & 0.319 & 0.286 & 0.265 & 1.375 & 0.785 & 2.409 \\
\hline \multicolumn{7}{|c|}{ Year of index prescription (ref: 2001) } \\
\hline - 1998 & -0.607 & 0.385 & 0.115 & 0.545 & 0.256 & 1.160 \\
\hline - 1999 & -0.329 & 0.358 & 0.358 & 0.720 & 0.357 & 1.451 \\
\hline - 2000 & 0.043 & 0.343 & 0.901 & 1.044 & 0.532 & 2.045 \\
\hline \multicolumn{7}{|c|}{ Co-morbid conditions in the pre period } \\
\hline Number of ER/hosp visits & 0.108 & 0.033 & $0.001 *$ & 1.114 & 1.044 & 1.189 \\
\hline Total health costs & 0.000 & 0.000 & $0.018^{*}$ & 1.000 & 1.000 & 1.000 \\
\hline Charlson co-morbidity & -0.200 & 0.102 & $0.048^{*}$ & 0.818 & 0.671 & 0.999 \\
\hline $\begin{array}{l}\text { Number of therapeutic classes } \\
\text { of medications }\end{array}$ & 0.137 & 0.076 & 0.072 & 1.147 & 0.988 & 1.333 \\
\hline Cardio-vascular & -0.172 & 0.158 & 0.276 & 0.842 & 0.617 & 1.148 \\
\hline Cancer & 0.221 & 0.403 & 0.583 & 1.248 & 0.567 & 2.747 \\
\hline Asthma & 0.191 & 0.224 & 0.396 & 1.210 & 0.779 & 1.879 \\
\hline Ulcers & 0.623 & 0.373 & 0.095 & 1.865 & 0.897 & 3.878 \\
\hline \multicolumn{7}{|c|}{ Provider interaction in the pre period } \\
\hline PAAS enrollment & 0.289 & 0.199 & 0.147 & 1.335 & 0.904 & 1.970 \\
\hline \multicolumn{7}{|c|}{ Number of pharmacies visited (ref: multiple pharmacies) } \\
\hline - No pharmacy & 0.022 & 0.392 & 0.955 & 1.022 & 0.474 & 2.205 \\
\hline - Single pharmacy & -0.077 & 0.144 & 0.590 & 0.926 & 0.698 & 1.227 \\
\hline
\end{tabular}


Table 9: Extended Cox proportional hazards model for the impact of depression on time to modification of oral hypoglycemic agent (Contd.)

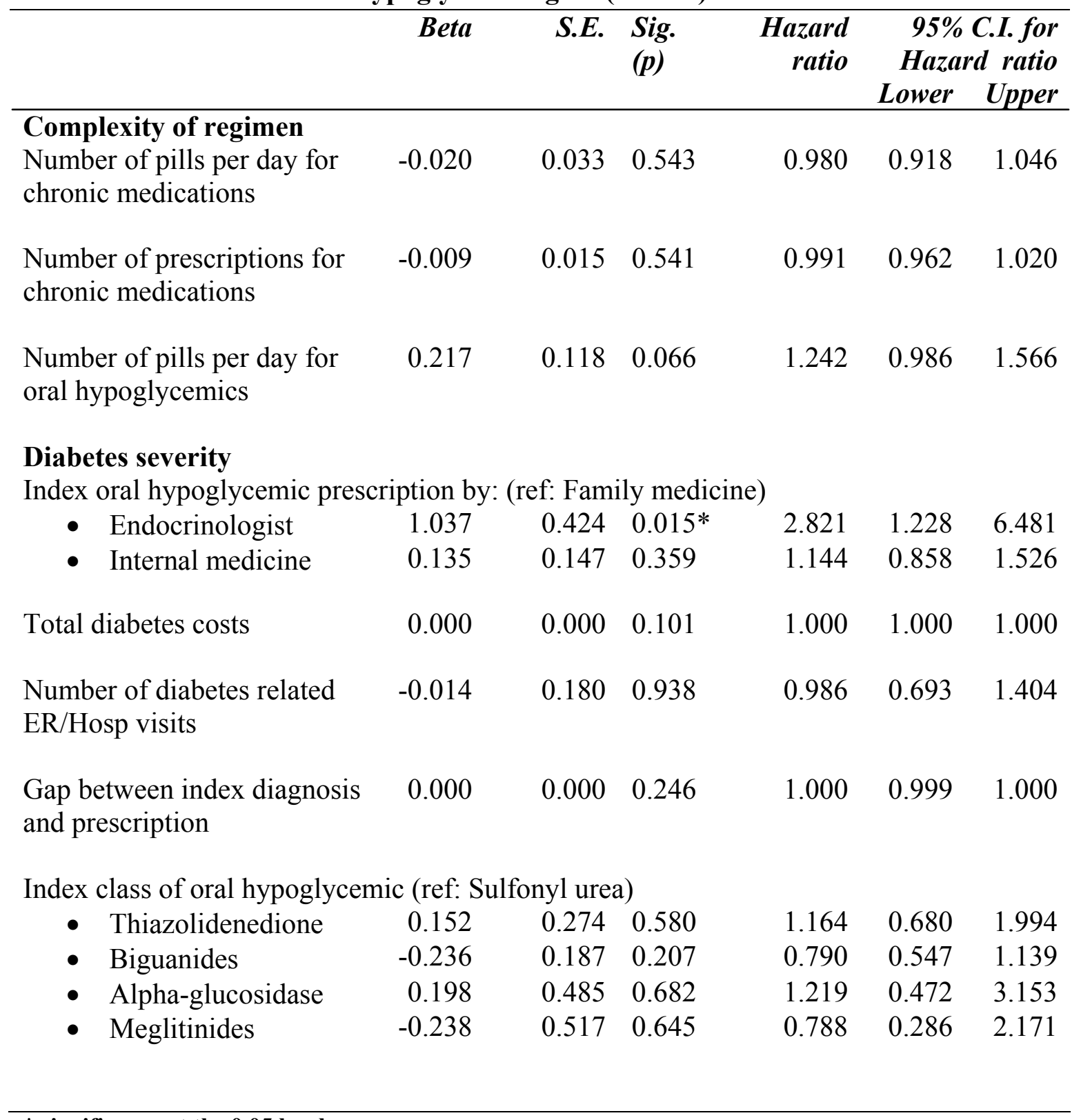

* significance at the 0.05 level

PAAS = Physician Assured Access System

\section{Model fit statistics:}

-2 Log Likelihood $=2863.587 ; \chi^{2}=72.07, p=0.001$

Global test of proportional hazards $: \chi^{2}=43.45, p=0.467$ 


\section{Discussion for objective 4}

Modification to treatment regimens may be indicative of poor blood glucose control, lack of tolerance of the drug, or the onset of concomitant conditions. Failure to achieve glycemic control or the onset of co-morbid conditions may prompt physicians to step up the treatment regimen by: (1) substituting a more potent medication or (2) adding another oral hypoglycemic agent, or (3) initiating insulin therapy.

Depression can decrease adherence with medications leading to inadequate glucose control and thus lead to modification of oral hypoglycemic therapy. The study results indicated the same with a significantly higher percent of depressed patients modifying their index oral hypoglycemic therapy as compared to non-depressed patients. No studies examining the impact of depression on modification of therapy are available in the literature. However, one can compare our overall rates of modification with findings from other studies. Results from the study conducted by Donnan and associates $^{17}$ in Tayside Scotland, indicated that around $28.00 \%$ of newly diagnosed type 2 diabetes patients modified their index oral hypoglycemic therapy over the median follow up time period of 588 days. A similar rate for modification of therapy (32.00\%) was observed over a 12-month follow up period in a study conducted in Medi-Cal. ${ }^{90}$ A more recent study conducted by Bocuzzi and associates ${ }^{16}$ in a large administrative dataset used a composite measure of change in therapy including switching or augmentation and found that a striking $36.00 \%$ of the patients changed therapy over a 12-month follow up period with a crude time to modification of therapy being around 170 days. Overall rates from this study for modification of $29.00 \%$ were similar to findings from previous studies. However, time to modification of therapy was longer (300 days) in this study as 
compared to the crude rates estimated from the study by Bocuzzi and associates ${ }^{16}(\sim 170$ days).

Multivariate results indicated that controlling for baseline characteristics, patients with depression were nearly 1.8 times more likely to modify index therapy as compared to non-depressed patients. Similarly, the hazard to modify therapy was 2.4 times more for depressed patients as compared to non-depressed patients in the latter six-month follow-up period.

\section{$\underline{\text { Results for Objective } 5}$}

To estimate the impact of pre-existing depression on initiation of insulin therapy in patients with type 2 diabetes

The univariate results are presented in Table 10. Results indicated that a significantly higher percent of depressed patients (5.30\%) initiated insulin therapy as compared to non-depressed patients $(2.90 \%)$. Higher rates of insulin initiation were also accompanied with a relatively shorter time period to begin insulin therapy for depressed patients (355.92 days) compared to non-depressed patients (358.71 days). A univariate Kaplan Meier analysis indicated that this time to insulin initiation was significantly higher for depressed patients with a log rank test statistic of $4.68(\mathrm{p}=0.030)$. These results can also be seen in terms of a better survival distribution for non-depressed patients in the Kaplan Meier survival curves for time to initiation of insulin therapy (Figure 7).

However, these differences between the two groups were not found to be significant in the multivariate framework after adjusting for confounders such as 
demographics, co-morbidity, diabetes severity, complexity of regimen, and interaction with health care providers. The logistic regression model to predict the impact of depression on the likelihood of initiating insulin therapy was found to be significant with a Nagelkerke R-square value of $14.40 \%$. The model results are summarized in Table 11 . Results of the multivariate model indicated that controlling for confounding covariates, there were no significant differences in the rate of initiation of insulin therapy in the 12month follow up period. The Cox proportional hazards model was found to be significant and satisfied the assumptions of proportional hazards. The model fit and results are summarized in Table 12. The model indicated that the time to initiate insulin therapy was not significantly different between depressed and non-depressed patients after adjusting for baseline covariates. 
Table 10: Initiation of insulin therapy in the 12-month follow up period

\begin{tabular}{|c|c|c|c|c|c|}
\hline & $\begin{array}{c}\text { Depressed } \\
(n=471)\end{array}$ & $\begin{array}{c}\text { Non-depressed } \\
(n=855)\end{array}$ & $\begin{array}{c}\text { Total } \\
(n=1326)\end{array}$ & $\begin{array}{c}\text { Test } \\
\text { statistic }\end{array}$ & $\begin{array}{l}\text { Sig. } \\
(p)\end{array}$ \\
\hline $\begin{array}{l}\text { Initiation of insulin thera } \\
\qquad \quad \text { Insulin use }\end{array}$ & $\begin{array}{l}5.30 \% \\
(25)\end{array}$ & $\begin{array}{l}2.90 \% \\
(25)\end{array}$ & $\begin{array}{l}3.80 \% \\
(50)\end{array}$ & & \\
\hline - No insulin use & $\begin{array}{l}94.70 \% \\
(446)\end{array}$ & $\begin{array}{l}97.10 \% \\
(830)\end{array}$ & $\begin{array}{l}96.20 \% \\
(1276)\end{array}$ & $\chi^{2}=4.76$ & $0.030 *$ \\
\hline $\begin{array}{l}\text { Time to initiation of } \\
\text { insulin } \\
\text { (in years) } \\
\text { (Mean }+ \text { Standard Error) }\end{array}$ & $\begin{array}{r}355.92 \\
( \pm 2.09)\end{array}$ & $\begin{array}{l}358.71 \\
( \pm 1.43)\end{array}$ & $\begin{array}{l}357.71 \\
( \pm 1.17)\end{array}$ & $\mathrm{LR}=4.68$ & $0.032 *$ \\
\hline
\end{tabular}

*significance at the 0.05 level

$\chi^{2}=$ Chi-square test

LR $=$ Log Rank test 
Figure 7: Kaplan-Meier Survival Curves for time to initiation of insulin therapy

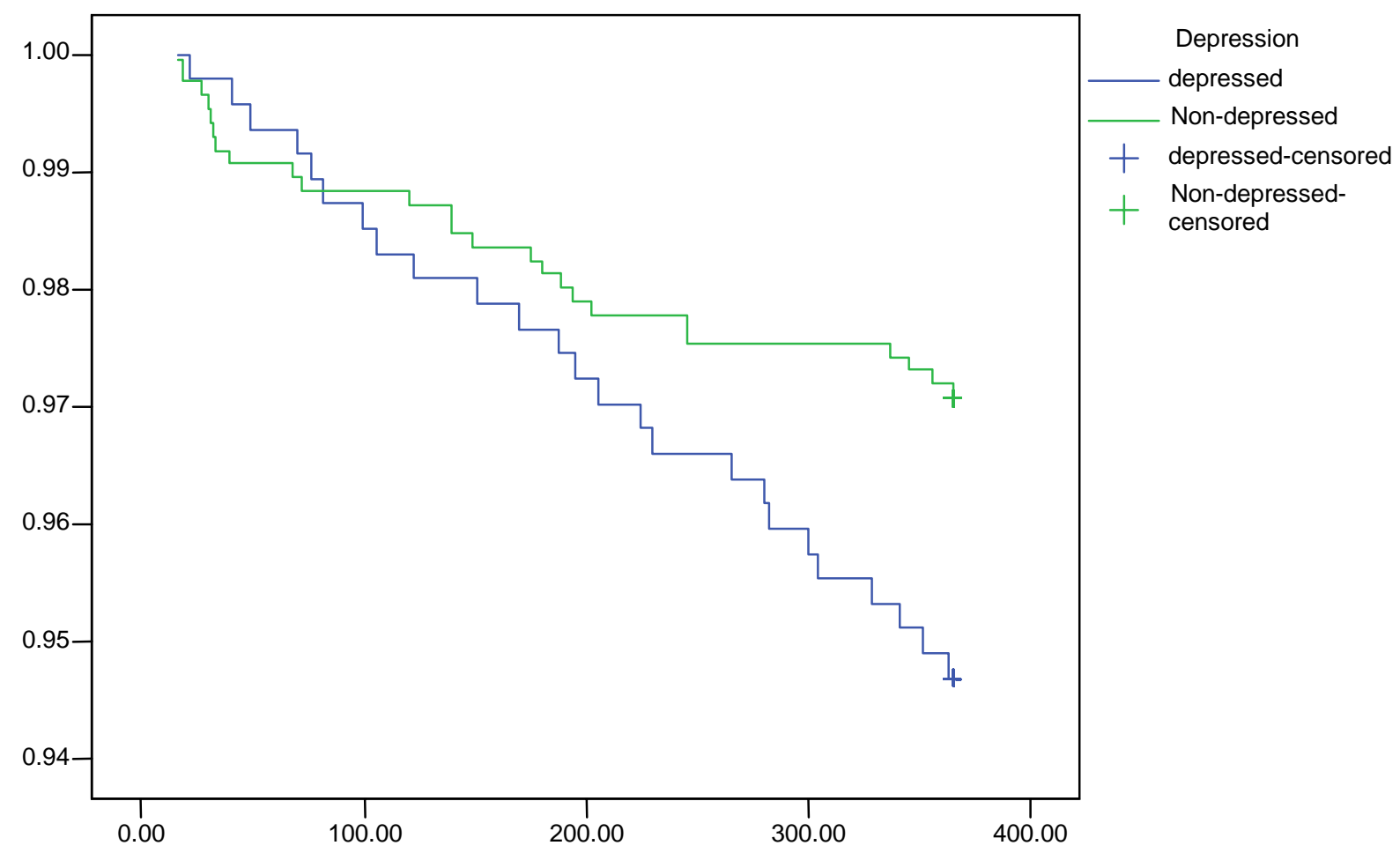

Time to initiation of insulin therapy in the 12-month follow up 
Table 11: Logistic regression for the impact of depression on initiation of insulin therapy

\begin{tabular}{|c|c|c|c|c|c|c|}
\hline & \multirow[t]{2}{*}{ Beta } & \multirow[t]{2}{*}{ S.E. } & \multirow[t]{2}{*}{$\begin{array}{l}\text { Sig. } \\
\text { (p) }\end{array}$} & \multirow[t]{2}{*}{$\begin{array}{l}\text { Odds } \\
\text { ratio }\end{array}$} & \multicolumn{2}{|c|}{$\begin{array}{r}95 \% \text { C.I. for } \\
\text { Odds ratio }\end{array}$} \\
\hline & & & & & Lower & Upper \\
\hline Depression & 0.521 & 0.341 & 0.126 & 1.684 & 0.864 & 3.283 \\
\hline \multicolumn{7}{|l|}{ Demographic characteristics } \\
\hline Age (in years) & -0.015 & 0.017 & 0.355 & 0.985 & 0.953 & 1.017 \\
\hline Males (ref: females) & -0.164 & 0.349 & 0.637 & 0.848 & 0.428 & 1.680 \\
\hline Whites (ref: non-whites) & 1.029 & 1.040 & 0.323 & 2.798 & 0.364 & 21.505 \\
\hline Urban (ref: rural) & 0.243 & 0.588 & 0.679 & 1.275 & 0.403 & 4.039 \\
\hline \multicolumn{7}{|c|}{ Year of index prescription (ref: 2001) } \\
\hline - 1998 & -0.449 & 0.908 & 0.621 & 0.638 & 0.108 & 3.785 \\
\hline - 1999 & 0.363 & 0.826 & 0.660 & 1.438 & 0.285 & 7.255 \\
\hline - 2000 & -0.096 & 0.807 & 0.905 & 0.908 & 0.187 & 4.421 \\
\hline \multicolumn{7}{|c|}{ Co-morbid conditions in the pre period } \\
\hline Number of ER/hosp visits & 0.005 & 0.047 & 0.920 & 1.005 & 0.916 & 1.102 \\
\hline Total health costs & 0.000 & 0.000 & 0.200 & 1.000 & 1.000 & 1.000 \\
\hline Charlson co-morbidity & 0.292 & 0.158 & 0.064 & 1.340 & 0.984 & 1.825 \\
\hline $\begin{array}{l}\text { Number of therapeutic classes } \\
\text { of medications }\end{array}$ & 0.255 & 0.163 & 0.117 & 1.290 & 0.938 & 1.775 \\
\hline Cardio-vascular & -0.455 & 0.350 & 0.194 & 0.634 & 0.319 & 1.261 \\
\hline Cancer & -2.187 & 1.281 & 0.088 & 0.112 & 0.009 & 1.381 \\
\hline Asthma & -0.498 & 0.457 & 0.276 & 0.608 & 0.248 & 1.489 \\
\hline Ulcers & -1.009 & 1.098 & 0.358 & 0.365 & 0.042 & 3.137 \\
\hline \multicolumn{7}{|c|}{ Provider interaction in the pre period } \\
\hline PAAS enrollment & 0.169 & 0.448 & 0.705 & 1.185 & 0.492 & 2.852 \\
\hline \multicolumn{7}{|c|}{ Number of pharmacies visited (ref: multiple pharmacies) } \\
\hline - No pharmacy & 0.517 & 0.644 & 0.422 & 1.677 & 0.474 & 5.930 \\
\hline - Single pharmacy & -0.759 & 0.350 & $0.030^{*}$ & 0.468 & 0.236 & 0.929 \\
\hline
\end{tabular}


Table 11: Logistic regression for the impact of depression on initiation of insulin therapy (Contd.)

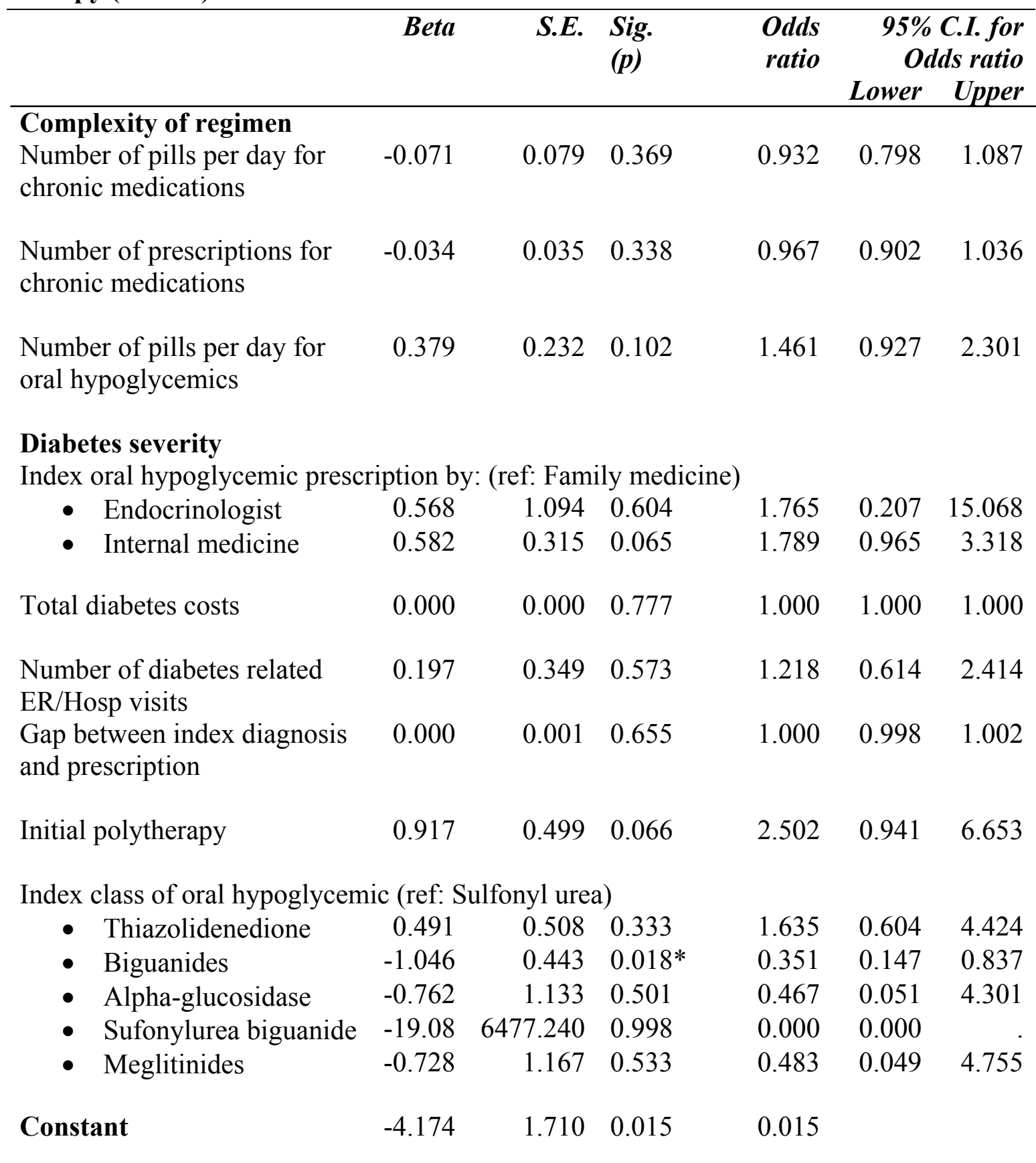

*significance at 0.05 level

PAAS = Physician Assured Access System

Model fit statistics:

Pseudo R-square $=14.4 \%$

-2 Log Likelihood $=370.174 ; \chi^{2}=53.058, p=0.015$

Hosmer and Lemeshow's goodness of fit: $\chi^{2}=2.828 ; p=0.945$ 
Table 12: Cox proportional hazards model for the impact of depression on time to initiation of insulin therapy

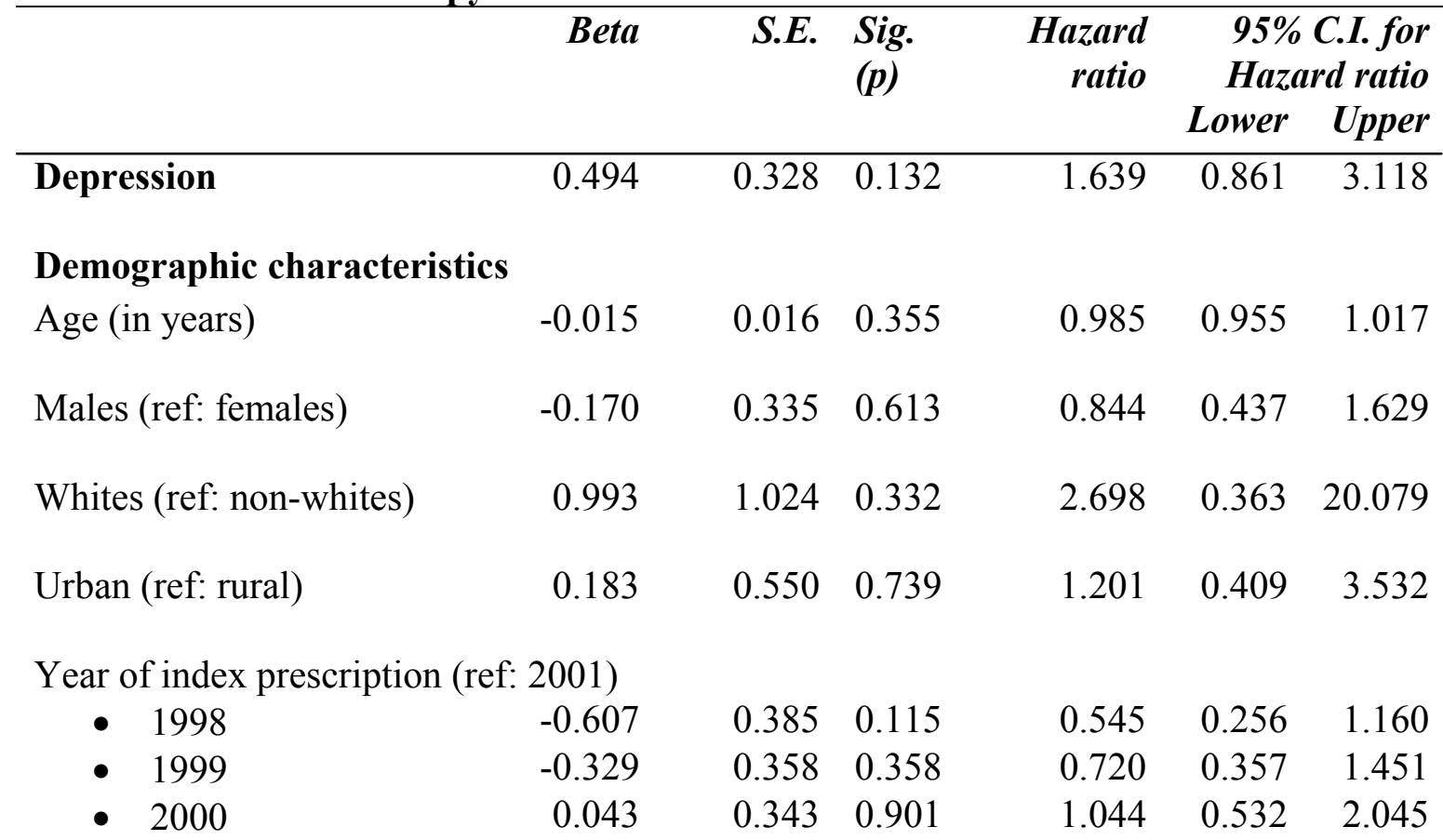

Co-morbid conditions in the pre period

Number of ER/hosp visits $\quad 0.000$

$\begin{array}{lllll}0.042 & 0.997 & 1.000 & 0.921 & 1.086\end{array}$

Total health costs

$\begin{array}{llllll}0.000 & 0.000 & 0.241 & 1.000 & 1.000 & 1.000\end{array}$

Charlson co-morbidity

$0.260 \quad 0.144 \quad 0.071$

$\begin{array}{lll}1.297 & 0.978 & 1.719\end{array}$

Number of therapeutic classes $\quad 0.238$

$0.155 \quad 0.124$

$\begin{array}{lll}1.269 & 0.937 & 1.719\end{array}$

of medications

Cardio-vascular

$\begin{array}{llllll}-0.414 & 0.334 & 0.215 & 0.661 & 0.343 & 1.272\end{array}$

Cancer

$-1.997$

$1.229 \quad 0.104$

$\begin{array}{lll}0.136 & 0.012 & 1.510\end{array}$

Asthma

$-0.435$

$0.431 \quad 0.314$

$\begin{array}{lll}0.647 & 0.278 & 1.508\end{array}$

Ulcers

$-0.948$

$1.067 \quad 0.375$

$\begin{array}{lll}0.388 & 0.048 & 3.138\end{array}$

Provider interaction in the pre period

PAAS enrollment

0.176

$0.429 \quad 0.681$

$1.193 \quad 0.515$

2.763

Number of pharmacies visited (ref: multiple pharmacies)
- No pharmacy
0.487
$\begin{array}{ll}0.616 & 0.428\end{array}$
$\begin{array}{lll}1.628 & 0.487 & 5.441\end{array}$
- Single pharmacy
$-0.718$
$0.336 \quad 0.033^{*}$
$\begin{array}{lll}0.488 & 0.252 & 0.942\end{array}$ 
Table 12: Cox proportional hazards model for the impact of depression on time to initiation of insulin therapy (Contd.)

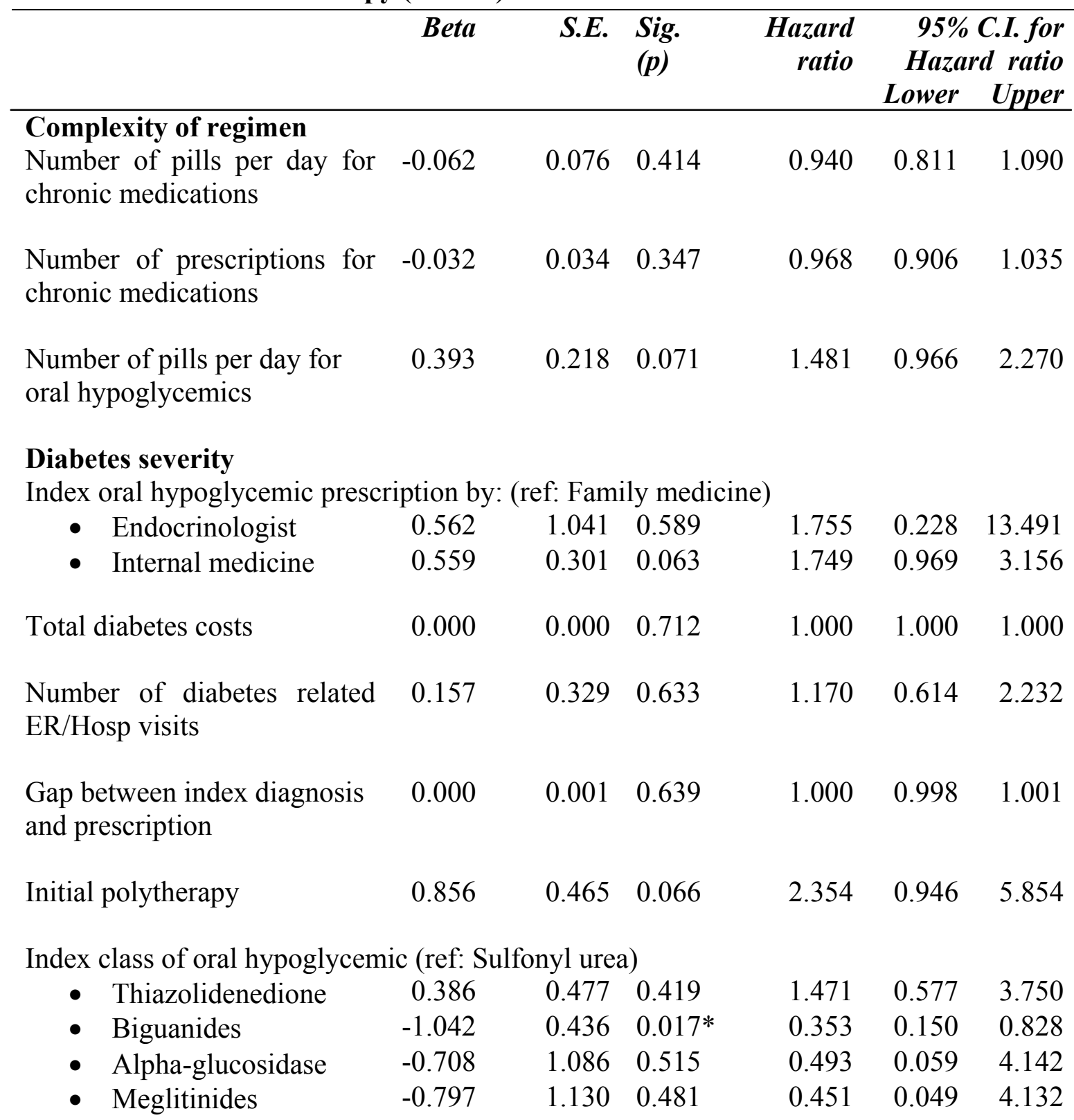

*significance at the 0.05 level

PAAS = Physician Assured Access System

Model fit statistics:

-2 Log Likelihood $=662.481 ; \chi^{2}=54.049, p=0.012$

Global test of proportional hazard: $\chi^{2}=31.86 ; p=0.5237$ 


\section{Discussion for Objective 5}

Initiation of insulin therapy can be a proxy for failure to control blood glucose with oral hypoglycemic therapy. Depression can decrease adherence with medications leading to inadequate glucose control and cause a subsequent need for insulin therapy. Although significant differences were observed at the univariate level, these differences were not seen when the objective was examined in a multivariate framework. One reason for this may be the length of the follow up period. Usually insulin therapy is initiated when oral hypoglycemics and their combinations fail to control blood glucose and is not seen in a large fraction of newly diagnosed type 2 diabetes within a year of therapy.

There are no studies in literature examining the role of depression in initiation of insulin therapy in patients with type 2 diabetes. However, studies have examined the issue of insulin initiation extensively. These studies have reported a high variation in rates of progression to insulin therapy in newly diagnosed patients. This was primarily due to studies having a small sample sizes and being conducted in specialized settings such as diabetes clinics. In a study conducted by Spoelstar and associates ${ }^{161}$ in a Dutch general health care system, the cumulative incidence of switching to insulin therapy was $36 \%$ over a 4.5-year period. Results from the United Kingdom Prospective Database study report insulin initiation rate of $30 \%$ over a nine year follow up period. ${ }^{162}$ One of the large population based studies examining this issue was conducted by Donnan and associates ${ }^{163}$ using retrospective claims data in incident type 2 diabetes patients in Tayside, Scotland. The newly diagnosed type 2 diabetes had varying times of follow up with a median length for the total cohort being 588 days. Overall, $9.40 \%$ of subjects switched to insulin with a median time of switching being approximately six months after 
initiation of oral hypoglycemic therapy. The annual rate of switching was computed to be 5.84\%. These rates in literature are comparable to annual insulin initiation rate of $3.80 \%$ found in this study.

\section{$\underline{\text { Results for Objective } 6}$}

To estimate the impact of pre-existing depression on adherence to oral hypoglycemic agents

Adherence to oral hypoglycemics was computed using refill information from claims data. Patients initiating either mono-therapy or poly-therapy involving insulin were excluded from the computation of adherence indices. All insulin prescriptions were excluded from the calculation of adherence indices. This objective was examined using different indices for adherence such as the Reg MPR-1 and Reg MPR-2. Univariate ttests were conducted to examine the differences in adherence indices.

Univariate results are presented in Table 13. Results indicated that patients with depression had significantly lower adherence to oral hypoglycemics than non-depressed patients on the basis of both Reg MPR-1 and Reg MPR-2. However, although the differences were significant the magnitude of difference was not large with patients with depression being only $3 \%$ and $6 \%$ less adherent to oral hypoglycemics than nondepressed patients on the Reg MPR-1 and Reg MPR-2 index respectively. A multivariate Ordinary Least Squares (OLS) regression was performed to determine the impact of depression on adherence (Reg MPR-1 and Reg MPR-2) to oral hypoglycemic use controlling for baseline confounding factors. Results of the multivariate OLS models are presented in Tables 14 and 15 . The multivariate results were exactly similar to the 
univariate analysis wherein the depressed patients were found to be significantly less adherent than non-depressed patients. The magnitude of difference in adherence after adjusting for baseline covariates was similar to unadjusted differences with depressed patients being approximately $3 \%$ and $7 \%$ less adherent than non-depressed patients as measured by Reg MPR-1 and Reg MPR-2.

However, this multivariate OLS model was estimated on the assumption that depression has a linear effect on adherence. Also, in objective 9, which estimates the impact of adherence on health care expenditures, a continuous adherence score could not be used as the independent variable as literature indicates a possible non-linear relationship between adherence to medications and health care expenditures. Hence, we categorized Reg MPR-1 and Reg MPR-2 to examine the impact of depression on categories of adherence levels. Categories of adherence were computed on the basis of recommendations from clinical experts and on the basis of distribution of adherence rates in the study data.

Adherence measured by both Reg MPR-1 and Reg MPR-2 was divided into the following categories:

- Very low adherence: (Reg MPR-1 and Reg MPR-2 greater than or equal to 0 and less than or equal to 0.55 )

- Low adherence: (Reg MPR-1 and Reg MPR-2 greater than 0.55 and less than or equal to 0.7 )

- Average adherence: (Reg MPR-1 and Reg MPR-2 greater than 0.70 and less than or equal to 0.85 ) 
- Good adherence: (Reg MPR-1 and Reg MPR-2 greater than 0.85 and less than or equal to 1.0$)$

- Over adherence: (Reg MPR-1 and Reg MPR-2 greater than 1.0).

Univariate distributions of depressed and non-depressed patients on the basis of these categories are presented in Table 13.

As multiple categories of adherence were created, multivariate multinomial logistic regression analysis was used to estimate the impact of preexisting depression on these categories of adherence controlling for other confounding factors. Good adherence was used as the reference category for all comparisons in the multinomial logistic model framework. Separate multinomial logistic regression models were estimated for the impact of depression on Reg MPR-1 and Reg MPR-2. The multinomial logistic regression model for the impact of depression on Reg MPR-1 and Reg MPR-2 were found to be significant with a Nagelkerke R-square value of $26.10 \%$ and $20.00 \%$, respectively. The model results are summarized in Tables 16A-16D and Table 17A-17D. Results of the multivariate model for Reg MPR-1 indicated that controlling for confounding covariates, patients with depression were 1.9 times more likely to have low adherence to oral hypoglycemics as compared non-depressed patients in the 12 month follow up period $(p=0.031)$. Results from the model for Reg MPR-2 demonstrated that depressed patients were 1.6 times more likely to be in the very low adherence group as compared to non-depressed patients $(\mathrm{p}=0.004)$. No other comparisons in the multinomial logistic regression models were found to be significant. 
Table 13: Adherence indices in the 12-month follow up period

Reg MPR-1: Based on adherence between index prescription and the last oral hypoglycemic prescription fill

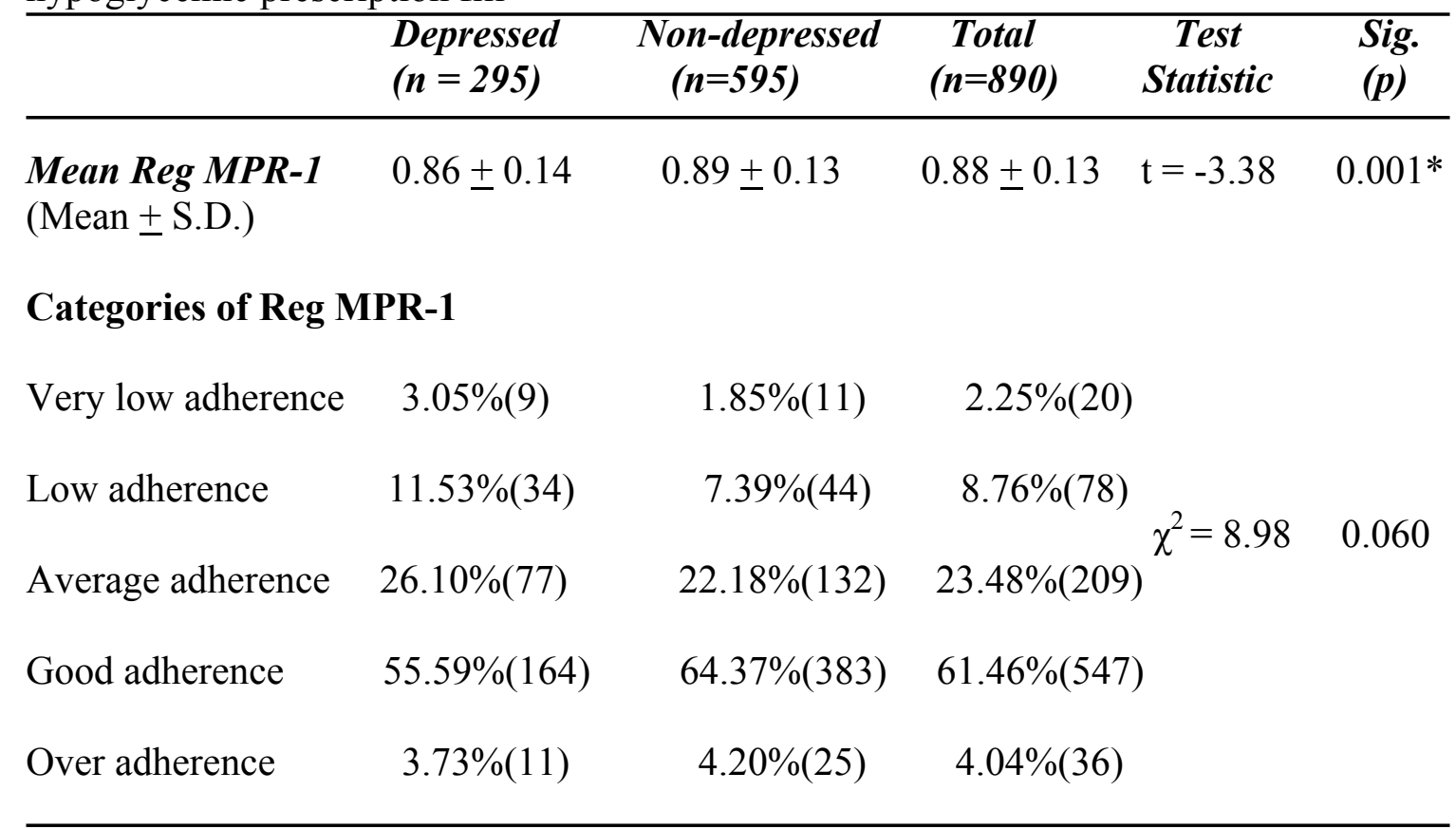

Reg MPR-2: Based on adherence between index prescription and the end of the follow up period

\begin{tabular}{lccccc}
\hline & $\begin{array}{c}\text { Depressed } \\
(\boldsymbol{n}=471)\end{array}$ & $\begin{array}{c}\text { Non-depressed } \\
(\boldsymbol{n}=\mathbf{8 5 5})\end{array}$ & $\begin{array}{c}\text { Total } \\
(\boldsymbol{n}=1326)\end{array}$ & $\begin{array}{c}\text { Test } \\
\text { Statistic }\end{array}$ & $\begin{array}{c}\text { Sig. } \\
(\boldsymbol{p})\end{array}$ \\
\hline $\begin{array}{l}\text { Mean Reg MPR-2 } \\
\text { (Mean } \pm \text { S.D.) }\end{array}$ & $0.66 \pm 0.31$ & $0.73 \pm 0.32$ & $0.73 \pm 0.34$ & $\mathrm{t}=-3.82$ & $0.000^{*}$ \\
& & & &
\end{tabular}

\section{Categories of Reg MPR-2}

Very low adherence $\quad 35.46 \%(167) \quad 27.02 \%(231) \quad 30.02 \%(398)$

Low adherence $\quad 12.31 \%(58) \quad 9.82 \%(84) \quad 10.71 \%(142)$

Average adherence $\quad 14.43 \%(68) \quad 14.15 \%(121) \quad 14.25 \%(189) \quad \chi^{2}=20.57 \quad 0.060$

Good adherence $\quad 32.70 \%(154) \quad 39.30 \%(336) \quad 24.51 \%(325)$

Over adherence $\quad 5.10 \%(24) \quad 9.71 \%(83) \quad 8.07 \%(107)$

* significance at the 0.05 level

$\mathbf{t}=\mathbf{t}$-test

$\chi^{2}=$ Chi-square test 
Table 14: Ordinary Least Squares (OLS) regression model for the impact of depression on adherence (Reg MPR-1) to oral hypoglycemics

$\begin{array}{lccc}\text { Beta } & \text { S.E. } & \begin{array}{c}\text { Test } \\ \text { statistic ( }(t)\end{array} & \begin{array}{l}\text { Sig. } \\ (p)\end{array} \\ \end{array}$

$\begin{array}{lllll}\text { Depression } & -0.028 & 0.010 & -2.880 & 0.004^{*}\end{array}$

Demographic characteristics

Age (in years)

$\begin{array}{llll}0.001 & 0.000 \quad 2.012 & 0.045^{*}\end{array}$

Males (ref: females)

$\begin{array}{llll}-0.013 & 0.009 & -1.381 & 0.168\end{array}$

Whites (ref: non-whites)

$0.061 \quad 0.020 \quad 3.061 \quad 0.002 *$

Urban (ref: rural)

$\begin{array}{llll}0.004 & 0.016 & 0.260 & 0.795\end{array}$

Year of index prescription (ref: 2001)
- 1998
0.013
0.023
$\begin{array}{ll}0.559 & 0.577\end{array}$
- 1999
$-0.003$
0.022
$\begin{array}{ll}-0.152 & 0.879\end{array}$
- 2000
$0.014 \quad 0.020$
$0.698 \quad 0.485$

Co-morbid conditions in the pre period

Number of ER/hosp visits

$\begin{array}{llll}-0.003 & 0.002 & -1.234 & 0.217 \\ 0.000 & 0.000 & 2.437 & 0.015^{*} \\ 0.006 & 0.006 & 1.167 & 0.244 \\ -0.027 & 0.005 & -5.627 & 0.000^{*} \\ & & & \\ -0.004 & 0.010 & -0.434 & 0.665 \\ -0.009 & 0.026 & -0.351 & 0.726 \\ -0.019 & 0.014 & -1.380 & 0.168 \\ 0.000 & 0.028 & -0.002 & 0.998\end{array}$

Total health costs

Charlson co-morbidity

Number of therapeutic classes

of medications

Cardio-vascular

Cancer

0.000

$\begin{array}{ll}0.017 & 0.014 \\ \text { ple pharmacies) }\end{array}$

$1.226 \quad 0.221$

Provider interaction

Number of pharmacies visited (ref: multiple pharmacies)
- No pharmacy
$-0.021$
0.024
$\begin{array}{ll}-0.892 & 0.373\end{array}$
- Single pharmacy
$0.004 \quad 0.009$
$0.496 \quad 0.620$ 
Table 14: Ordinary Least Squares (OLS) regression model for the impact of depression on adherence (Reg MPR-1) to oral hypoglycemics (Contd.)

\begin{tabular}{|c|c|c|c|c|}
\hline & Beta & S.E. & $\begin{array}{c}\text { Test } \\
\text { statistic (t) }\end{array}$ & $\begin{array}{l}\text { Sig. } \\
\text { (p) }\end{array}$ \\
\hline \multicolumn{5}{|l|}{ Complexity of regimen } \\
\hline $\begin{array}{l}\text { Number of pills per day for } \\
\text { chronic medications }\end{array}$ & 0.000 & 0.002 & -0.161 & 0.872 \\
\hline $\begin{array}{l}\text { Number of prescriptions for } \\
\text { chronic medications }\end{array}$ & 0.005 & 0.001 & 5.881 & $0.000^{*}$ \\
\hline $\begin{array}{l}\text { Number of pills per day for } \\
\text { oral hypoglycemics }\end{array}$ & -0.018 & 0.008 & -2.188 & $0.029 *$ \\
\hline \multicolumn{5}{|l|}{ Diabetes severity } \\
\hline \multicolumn{5}{|c|}{ Index oral hypoglycemic prescription by: (ref: Family medicine) } \\
\hline - Endocrinologist & 0.011 & 0.033 & 0.328 & 0.743 \\
\hline - Internal medicine & 0.004 & 0.010 & 0.402 & 0.688 \\
\hline Total diabetes costs & 0.000 & 0.000 & 1.971 & $0.049 *$ \\
\hline $\begin{array}{l}\text { Number of diabetes related } \\
\text { ER/Hosp visits }\end{array}$ & -0.015 & 0.013 & -1.156 & 0.248 \\
\hline $\begin{array}{l}\text { Gap between index diagnosis } \\
\text { and prescription }\end{array}$ & 0.000 & 0.000 & 0.387 & 0.699 \\
\hline Initial polytherapy & -0.050 & 0.017 & -2.978 & $0.003 *$ \\
\hline \multicolumn{5}{|c|}{ Index class of oral hypoglycemic (ref: Sulfonyl urea) } \\
\hline - Thiazolidenedione & -0.007 & 0.018 & -0.389 & 0.697 \\
\hline - Biguanides & -0.005 & 0.012 & -0.417 & 0.677 \\
\hline - Alpha-glucosidase & 0.008 & 0.036 & 0.226 & 0.821 \\
\hline - Meglitinides & 0.073 & 0.030 & 2.422 & $0.016^{*}$ \\
\hline Constant & 0.814 & 0.042 & 19.225 & 0.000 \\
\hline
\end{tabular}

*significance at the 0.05 level

PAAS: Physician Assured Access System

\section{Model fit statistics:}

R-square: $13.8 \% ; \mathrm{F}=4.026 ; \mathrm{P}=0.000$

Durbin-Watson: 1.980

White test for heteroskedasticity: Chi-square $=480.33 \mathrm{p}=0.7073$ 
Table 15: Ordinary Least Squares (OLS) regression model for the impact of depression on adherence (Reg MPR-2) to oral hypoglycemics

\section{Beta S.E. Test statistic Sig.}

(t)

(p)

Depression

Demographic characteristics

Age (in years)

Males (ref: females)

Whites (ref: non-whites)

Urban (ref: rural)
$-0.057$

$0.002 \quad 0.001$

$0.004 \quad 0.020$

$0.062 \quad 0.041$

0.018

Year of index prescription (ref: 2001)

- 1998

- 1999

- 2000
$0.064 \quad 0.050$

$0.102 \quad 0.047$

0.059

0.044

0.004

0.000

0.011

0.010

0.021

0.052

0.029

0.056

$-0.060$

0.018

0.029

$\begin{array}{ll}1.276 & 0.202\end{array}$

$2.170 \quad 0.030$ *

$1.320 \quad 0.187$

$2.1150 .035^{*}$

$0.209 \quad 0.834$

$1.512 \quad 0.131$

$0.527 \quad 0.598$

of medications

Cardio-vascular

$\begin{array}{llll}-0.075 & 0.052 & -1.433 & 0.152\end{array}$

Asthma

$\begin{array}{llll}-0.053 & 0.029 & -1.843 & 0.066\end{array}$

Ulcers

Provider interaction in the pre period

PAAS enrollment
0.018

0.029

$-4.7270 .000 *$

$2.4670 .014^{*}$

$-0.755 \quad 0.450$

$-1.700 \quad 0.089$

$1.406 \quad 0.160$

$-1.4330 .152$

Number of pharmacies visited (ref: multiple pharmacies)
- No pharmacy
$-0.057$
0.048
$-1.195 \quad 0.232$
- Single pharmacy
0.025
0.019
$1.276 \quad 0.202$ 
Table 15: Ordinary Least Squares (OLS) regression model for the impact of depression on adherence (Reg MPR-2) to oral hypoglycemics (Contd.)

\begin{tabular}{|c|c|c|c|c|}
\hline & Beta & S.E. & $\begin{array}{c}\text { Test statistic } \\
(t)\end{array}$ & $\begin{array}{l}\text { Sig. } \\
\text { (p) }\end{array}$ \\
\hline \multicolumn{5}{|l|}{ Complexity of regimen } \\
\hline $\begin{array}{l}\text { Number of pills per day for } \\
\text { chronic medications }\end{array}$ & -0.003 & 0.004 & -0.782 & 0.434 \\
\hline $\begin{array}{l}\text { Number of prescriptions for } \\
\text { chronic medications }\end{array}$ & 0.009 & 0.002 & 4.724 & $0.000^{*}$ \\
\hline $\begin{array}{l}\text { Number of pills per day for } \\
\text { oral hypoglycemics }\end{array}$ & -0.004 & 0.017 & -0.227 & 0.820 \\
\hline \multicolumn{5}{|l|}{ Diabetes severity } \\
\hline \multicolumn{5}{|c|}{ Index oral hypoglycemic prescription by: (ref: Family medicine) } \\
\hline - Endocrinologist & 0.089 & 0.075 & 1.185 & 0.236 \\
\hline - Internal medicine & -0.009 & 0.020 & -0.423 & 0.673 \\
\hline Total diabetes costs & 0.000 & 0.000 & 1.854 & 0.064 \\
\hline $\begin{array}{l}\text { Number of diabetes related } \\
\text { ER/Hosp visits }\end{array}$ & 0.013 & 0.027 & 0.487 & 0.626 \\
\hline $\begin{array}{l}\text { Gap between index diagnosis } \\
\text { and prescription }\end{array}$ & 0.000 & 0.000 & 1.509 & 0.131 \\
\hline Initial polytherapy & 0.007 & 0.041 & 0.167 & 0.867 \\
\hline \multicolumn{5}{|c|}{ Index class of oral hypoglycemic (ref: Sulfonyl urea) } \\
\hline - Thiazolidenedione & 0.004 & 0.040 & 0.101 & 0.919 \\
\hline - Biguanides & -0.057 & 0.025 & -2.287 & $0.022 *$ \\
\hline - Alpha-glucosidase & 0.091 & 0.078 & 1.162 & 0.245 \\
\hline - Meglitinides & -0.017 & 0.079 & -0.213 & 0.832 \\
\hline Constant & 0.473 & 0.089 & 5.306 & 0.000 \\
\hline
\end{tabular}

* significance at the 0.05 level

PAAS: Physician Assured Access System

Model fit statistics:

R-square: $11.4 \% ; \mathrm{F}=4.906 ; \mathrm{P}=0.000$

Durbin-Watson: 1.934

White test for heteroskedasticity: Chi-square $=540.83 ; \mathrm{p}=0.2963$ 
Table 16A: Multinomial Logistic regression for the impact of depression on adherence (Reg MPR-1) to oral hypoglycemics

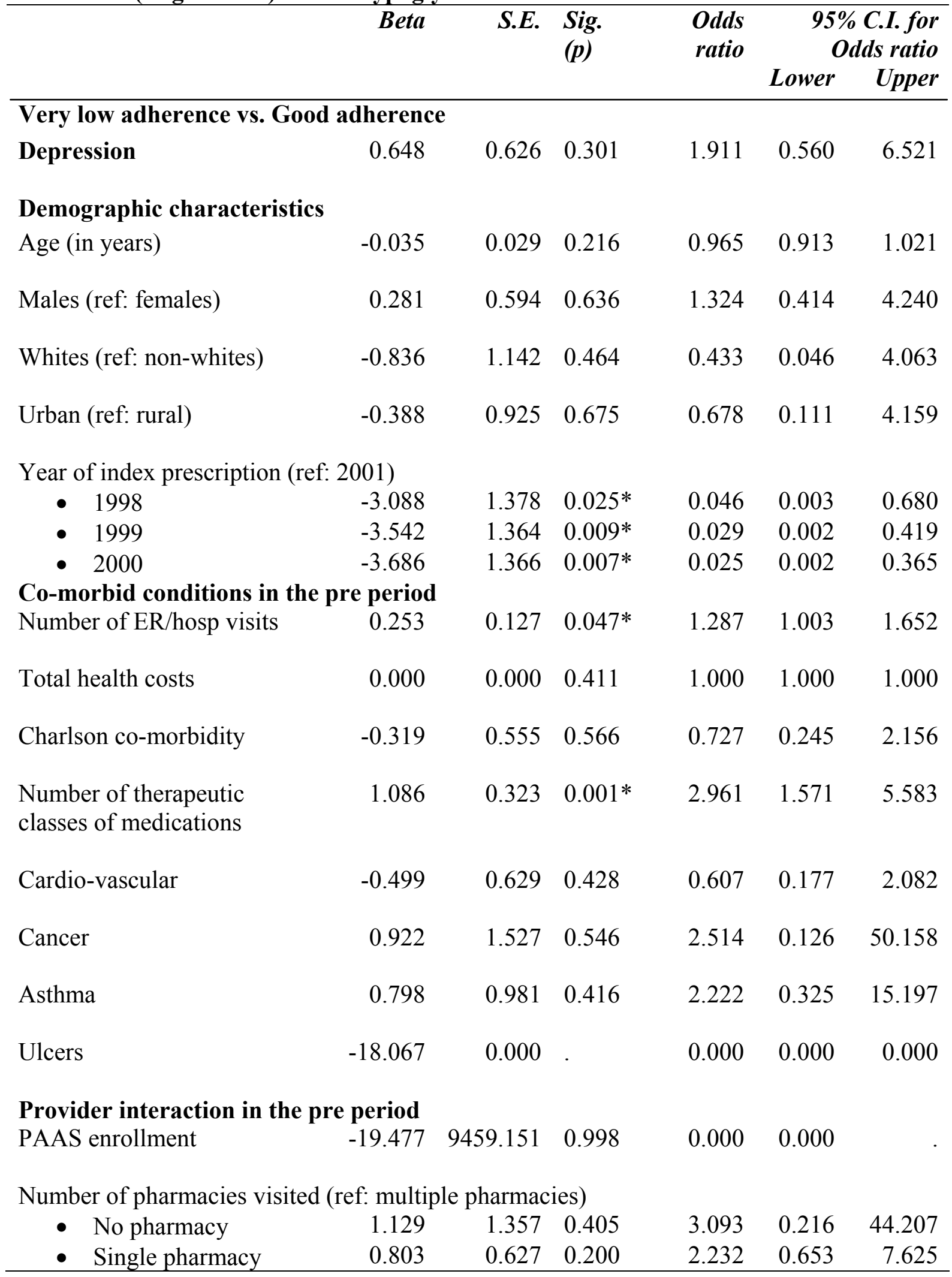


Table 16A: Multinomial Logistic regression for the impact of depression on adherence (Reg MPR-1) to oral hypoglycemics (Contd.)

\begin{tabular}{|c|c|c|c|c|c|c|}
\hline & \multirow[t]{2}{*}{ Beta } & \multirow[t]{2}{*}{ S.E. } & \multirow[t]{2}{*}{$\begin{array}{l}\text { Sig. } \\
\text { (p) }\end{array}$} & \multirow[t]{2}{*}{$\begin{array}{l}\text { Odds } \\
\text { ratio }\end{array}$} & \multicolumn{2}{|c|}{$\begin{array}{l}95 \% \text { C.I. for } \\
\text { Odds ratio }\end{array}$} \\
\hline & & & & & Lower & Upper \\
\hline \multicolumn{7}{|l|}{ Complexity of regimen } \\
\hline $\begin{array}{l}\text { Number of pills per day for } \\
\text { chronic medications }\end{array}$ & 0.045 & 0.147 & 0.758 & 1.046 & 0.784 & 1.396 \\
\hline $\begin{array}{l}\text { Number of prescriptions for } \\
\text { chronic medications }\end{array}$ & -0.265 & 0.076 & $0.001 *$ & 0.767 & 0.660 & 0.891 \\
\hline $\begin{array}{l}\text { Number of pills per day for } \\
\text { oral hypoglycemics }\end{array}$ & 0.684 & 0.381 & 0.073 & 1.983 & 0.939 & 4.187 \\
\hline \multicolumn{7}{|l|}{ Diabetes severity } \\
\hline \multicolumn{7}{|c|}{ Index oral hypoglycemic prescription by: (ref: Family medicine) } \\
\hline - Endocrinologist & -17.438 & 0.000 & 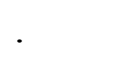 & 0.000 & 0.000 & 0.000 \\
\hline - Internal medicine & 0.050 & 0.632 & 0.937 & 1.051 & 0.304 & 3.628 \\
\hline Total diabetes costs & 0.000 & 0.000 & 0.879 & 1.000 & 1.000 & 1.000 \\
\hline $\begin{array}{l}\text { Number of diabetes related } \\
\text { ER/Hosp visits }\end{array}$ & -0.986 & 1.455 & 0.498 & 0.373 & 0.022 & 6.468 \\
\hline $\begin{array}{l}\text { Gap between index diagnosis } \\
\text { and prescription }\end{array}$ & -0.003 & 0.002 & 0.201 & 0.997 & 0.993 & 1.001 \\
\hline Initial polytherapy & 1.455 & 0.777 & 0.061 & 4.284 & 0.934 & 19.642 \\
\hline \multicolumn{7}{|c|}{ Index class of oral hypoglycemic (ref: Sulfonyl urea) } \\
\hline - Thiazolidenedione & -0.387 & 1.251 & 0.757 & 0.679 & 0.059 & 7.877 \\
\hline - Biguanides & 0.010 & 0.684 & 0.988 & 1.010 & 0.264 & 3.863 \\
\hline - Alpha-glucosidase & -18.235 & 0.000 & . & 0.000 & 0.000 & 0.000 \\
\hline - Sulfonyl-biguanides & -20.527 & 0.000 & & 0.000 & 0.000 & 0.000 \\
\hline - Meglitinides & 2.739 & 1.593 & 0.086 & 15.464 & 0.681 & 351.022 \\
\hline Constant & 0.631 & 2.535 & 0.804 & & & \\
\hline
\end{tabular}


Table 16B: Multinomial Logistic regression for the impact of depression on adherence (Reg MPR-1) to oral hypoglycemics

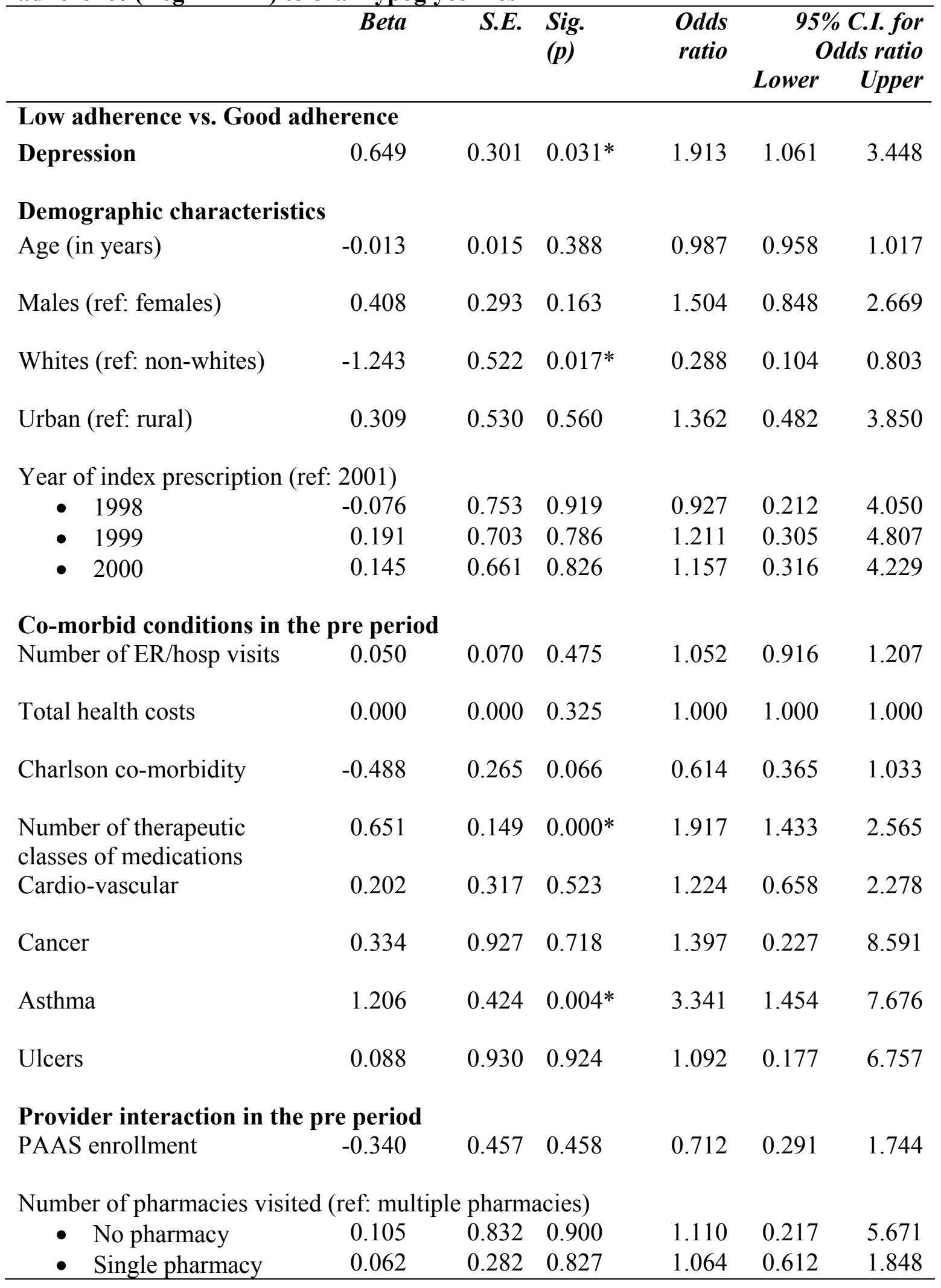


Table 16B: Multinomial Logistic regression for the impact of depression on adherence (Reg MPR-1) to oral hypoglycemics (Contd.)

\begin{tabular}{|c|c|c|c|c|c|c|}
\hline & \multirow[t]{2}{*}{ Beta } & \multirow[t]{2}{*}{ S.E. } & \multirow[t]{2}{*}{$\begin{array}{l}\text { Sig. } \\
(p)\end{array}$} & \multirow[t]{2}{*}{$\begin{array}{l}\text { Odds } \\
\text { ratio }\end{array}$} & \multicolumn{2}{|c|}{$\begin{array}{l}95 \% \text { C.I. for } \\
\text { Odds ratio }\end{array}$} \\
\hline & & & & & Lower & Upper \\
\hline \multicolumn{7}{|l|}{ Complexity of regimen } \\
\hline $\begin{array}{l}\text { Number of pills per day for } \\
\text { chronic medications }\end{array}$ & -0.039 & 0.070 & 0.575 & 0.962 & 0.839 & 1.102 \\
\hline $\begin{array}{l}\text { Number of prescriptions for } \\
\text { chronic medications }\end{array}$ & -0.110 & 0.031 & $0.000 *$ & 0.896 & 0.843 & 0.952 \\
\hline $\begin{array}{l}\text { Number of pills per day for } \\
\text { oral hypoglycemics }\end{array}$ & 0.568 & 0.220 & $0.010^{*}$ & 1.765 & 1.146 & 2.717 \\
\hline \multicolumn{7}{|l|}{ Diabetes severity } \\
\hline \multicolumn{7}{|c|}{ Index oral hypoglycemic prescription by: (ref: Family medicine) } \\
\hline - Endocrinologist & 1.060 & 0.926 & 0.252 & 2.886 & 0.470 & 17.720 \\
\hline - Internal medicine & 0.068 & 0.300 & 0.821 & 1.070 & 0.594 & 1.926 \\
\hline Total diabetes costs & 0.000 & 0.000 & 0.356 & 1.000 & 1.000 & 1.000 \\
\hline $\begin{array}{l}\text { Number of diabetes related } \\
\text { ER/Hosp visits }\end{array}$ & 0.383 & 0.431 & 0.374 & 1.467 & 0.630 & 3.418 \\
\hline $\begin{array}{l}\text { Gap between index diagnosis } \\
\text { and prescription }\end{array}$ & 0.000 & 0.001 & 0.795 & 1.000 & 0.999 & 1.002 \\
\hline Initial polytherapy & 1.393 & 0.418 & $0.001 *$ & 4.028 & 1.775 & 9.141 \\
\hline \multicolumn{7}{|c|}{ Index class of oral hypoglycemic (ref: Sulfonyl urea) } \\
\hline - Thiazolidenedione & 0.427 & 0.518 & 0.410 & 1.532 & 0.555 & 4.232 \\
\hline - Biguanides & 0.141 & 0.356 & 0.692 & 1.151 & 0.573 & 2.315 \\
\hline - Alpha-glucosidase & -0.802 & 1.284 & 0.532 & 0.448 & 0.036 & 5.555 \\
\hline - Sulfonyl-biguanides & -1.440 & 0.836 & 0.085 & 0.237 & 0.046 & 1.219 \\
\hline - Meglitinides & 1.569 & 1.112 & 0.158 & 4.801 & 0.543 & 42.439 \\
\hline Constant & -2.570 & 1.321 & 0.052 & & & \\
\hline
\end{tabular}


Table 16C: Multinomial Logistic regression for the impact of depression on adherence (Reg MPR-1) to oral hypoglycemics

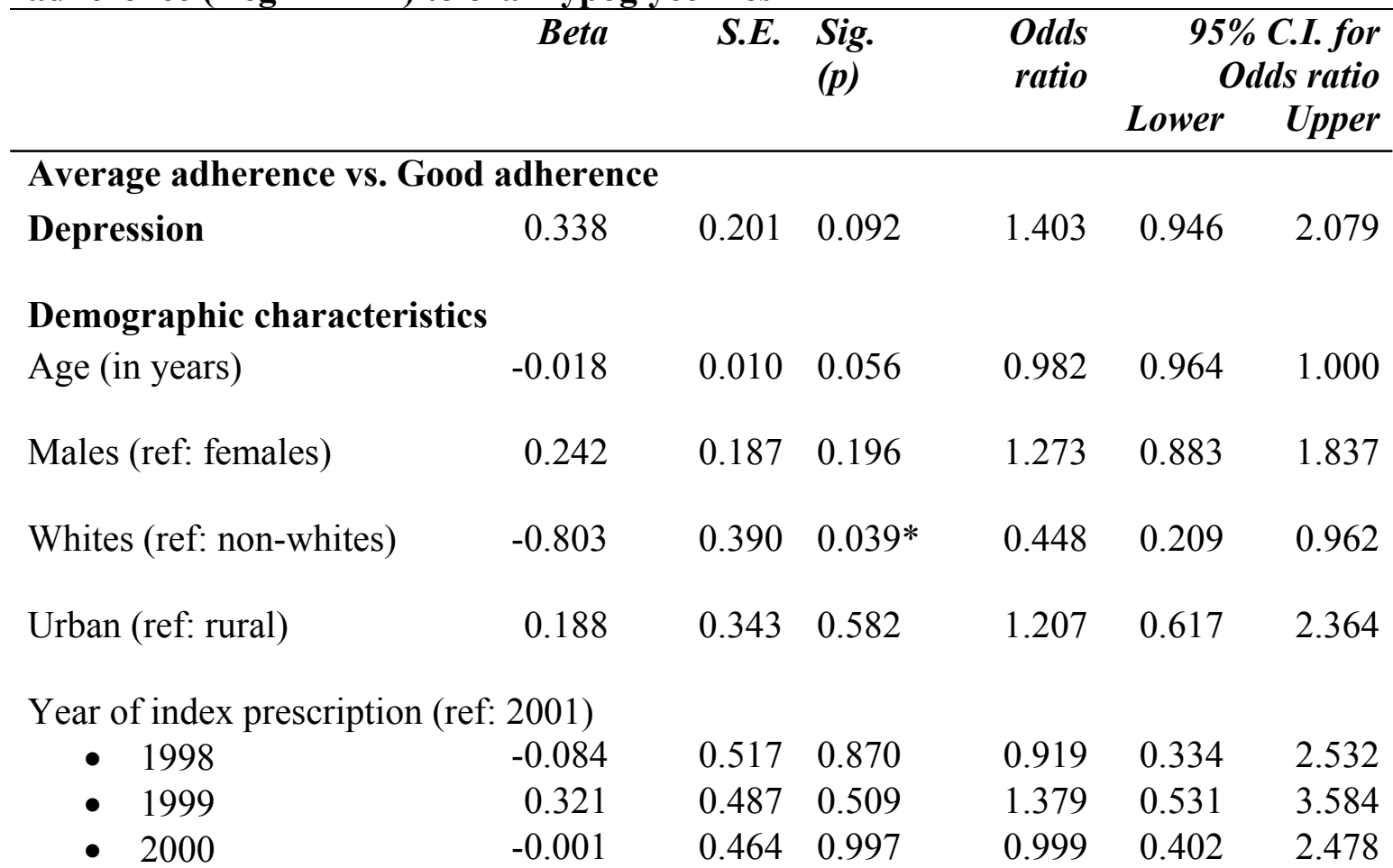

Co-morbid conditions in the pre period

Number of ER/hosp visits $\quad 0.069$

$\begin{array}{lllll}0.054 & 0.204 & 1.071 & 0.963 & 1.192\end{array}$

Total health costs

$\begin{array}{llllll}0.000 & 0.000 & 0.016^{*} & 1.000 & 1.000 & 1.000\end{array}$

Charlson co-morbidity

$-0.063$

$0.125 \quad 0.613$

$0.939 \quad 0.735$

1.199

Number of therapeutic

0.300

$0.103 \quad 0.004^{*}$

1.350

1.103

1.653

classes of medications

Cardio-vascular

$-0.181$

$0.203 \quad 0.374$

$0.835 \quad 0.560$

1.243

Cancer

0.388

$0.557 \quad 0.487$

$1.474 \quad 0.494$

4.394

Asthma

0.212

$0.291 \quad 0.467$

$1.236 \quad 0.698$

2.188

Ulcers

$-0.104$

$0.572 \quad 0.856$

0.901

0.294

2.764

Provider interaction in the pre period

PAAS enrollment $\quad 0.035$

$\begin{array}{lllll}0.266 & 0.896 & 1.035 & 0.615 & 1.742\end{array}$

Number of pharmacies visited (ref: multiple pharmacies)
- No pharmacy
0.196
0.4410 .658
$\begin{array}{ll}1.216 & 0.512\end{array}$
2.888
- Single pharmacy
$-0.145$
$0.184 \quad 0.433$
0.865
0.603
1.242 
Table 16C: Multinomial Logistic regression for the impact of depression on adherence (Reg MPR-1) to oral hypoglycemics (Contd.)

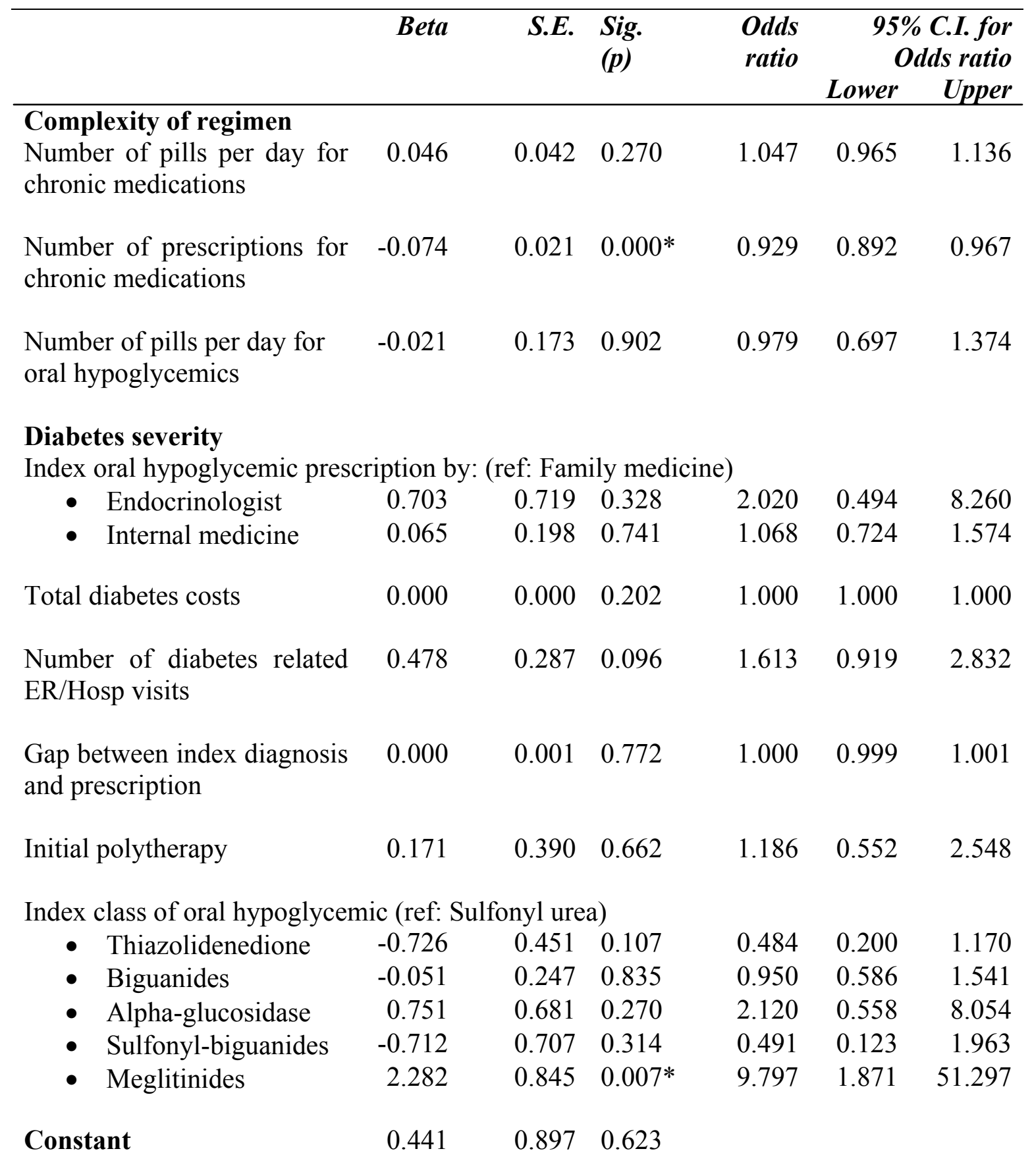


Table 16D: Multinomial Logistic regression for the impact of depression on adherence (Reg MPR-1) to oral hypoglycemics

$\begin{array}{llllr}\text { Beta } & \text { S.E. } & \text { Sig. } & \text { Odds } & 95 \% \text { C.I. for } \\ & (p) & \text { ratio } & \text { Odds ratio } \\ & & & \text { Lower } & \text { Upper }\end{array}$

Over adherence vs. Good adherence

Depression 0.304

$\begin{array}{llllll}0.304 & 0.422 & 0.472 & 1.355 & 0.593 & 3.097\end{array}$

Demographic characteristics

Age (in years)

$\begin{array}{llllll}-0.012 & 0.020 & 0.559 & 0.988 & 0.950 & 1.028\end{array}$

Males (ref: females)

$0.556 \quad 0.384 \quad 0.148$

$1.744 \quad 0.821$

3.704

Whites (ref: non-whites)

$15.777 \quad 0.000 \quad$.

Urban (ref: rural)

$\begin{array}{lll}1.293 & 1.051 & 0.218\end{array}$

$3.644 \quad 0.465$

28.588

Year of index prescription (ref: 2001)
- 1998
$-0.250$
$1.018 \quad 0.806$
0.779
0.106
5.730
- 1999
0.005
$0.954 \quad 0.996$
1.005
0.155
6.524
- 2000
$-0.102$
0.8930 .909
$0.903 \quad 0.157$
5.198

Co-morbid conditions in the pre period

Number of ER/hosp visits $\quad 0.040$

$0.118 \quad 0.736$

1.0410 .825

1.312

Total health costs

0.000

$0.000 \quad 0.648$

$1.000 \quad 1.000$

1.000

Charlson co-morbidity

$$
-0.210
$$

$0.313 \quad 0.502$

$0.811 \quad 0.439$

1.497

Number of therapeutic

$$
0.080
$$

$0.212 \quad 0.705$

1.083

0.716

1.640

classes of medications

Cardio-vascular

$-0.212$

$0.436 \quad 0.627$

0.809

0.344

1.902

Cancer

$-0.421$

$1.308 \quad 0.748$

$0.656 \quad 0.051$

8.527

Asthma

0.190

$0.616 \quad 0.758$

$1.209 \quad 0.362$

4.043

Ulcers

$\begin{array}{ll}-18.605 & 0.000\end{array}$

$0.000 \quad 0.000$

0.000

Provider interaction in the pre period

PAAS enrollment $\quad 0.078$

$0.582 \quad 0.894$

$1.081 \quad 0.346$

3.380

Number of pharmacies visited (ref: multiple pharmacies)
- No pharmacy
$-0.323$
1.151
0.779
$0.724 \quad 0.076$
6.906
- Single pharmacy
0.328
$0.399 \quad 0.412$
$1.388 \quad 0.634$
3.035 
Table 16D: Multinomial Logistic regression for the impact of depression on adherence (Reg MPR-1) to oral hypoglycemics (Contd.)

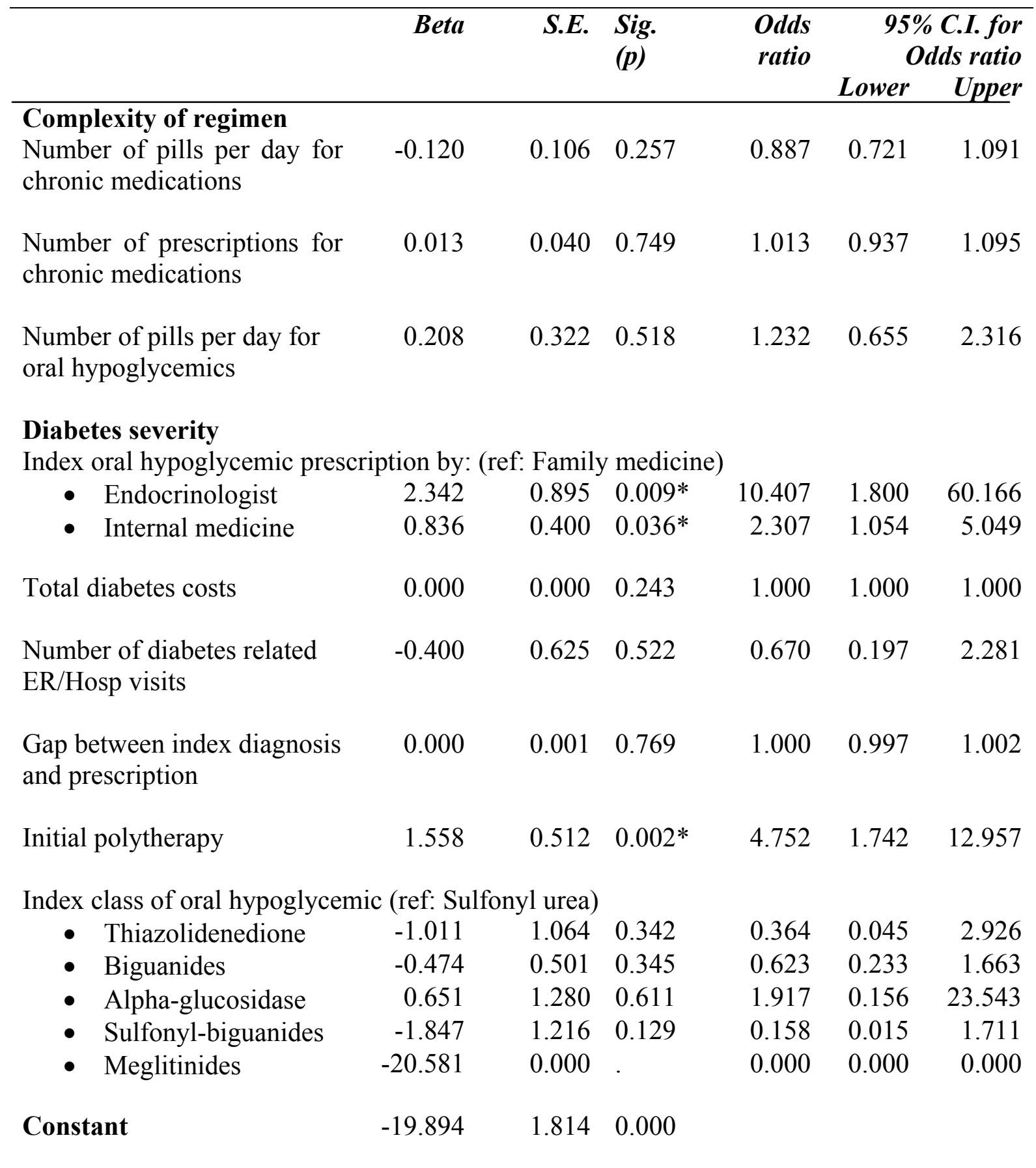

*significance at the 0.05 level

PAAS: Physician Assured Access System

Model fit statistics:

Pseudo R-square $=26.1 \%$

-2 Log Likelihood $=1609.302 ; \chi^{2}=226.003 ; p=0.00$ 
Table 17A: Multinomial Logistic regression for the impact of depression on adherence (Reg MPR-2) to oral hypoglycemics

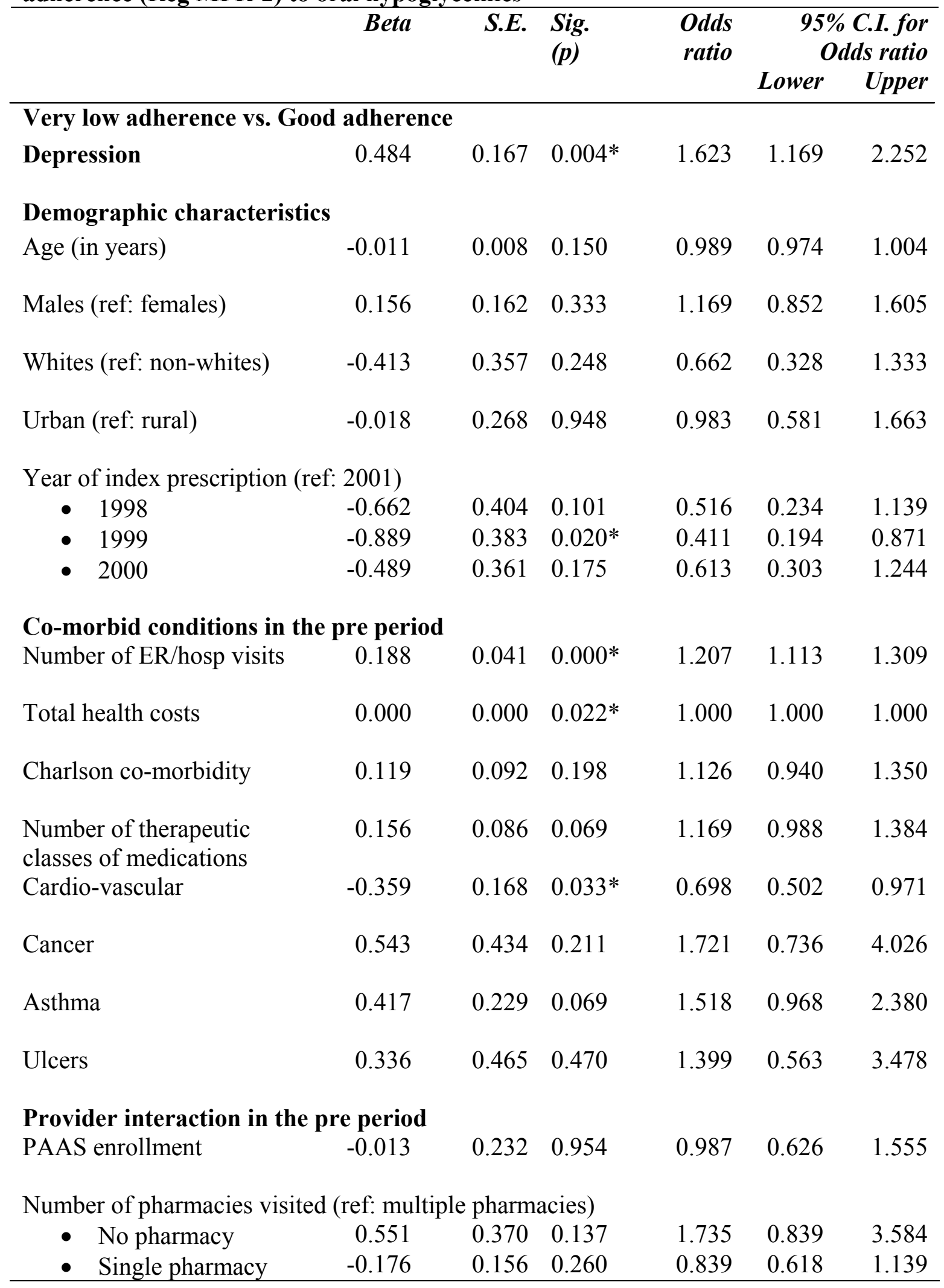


Table 17A: Multinomial Logistic regression for the impact of depression on adherence (Reg MPR-2) to oral hypoglycemics (Contd.)

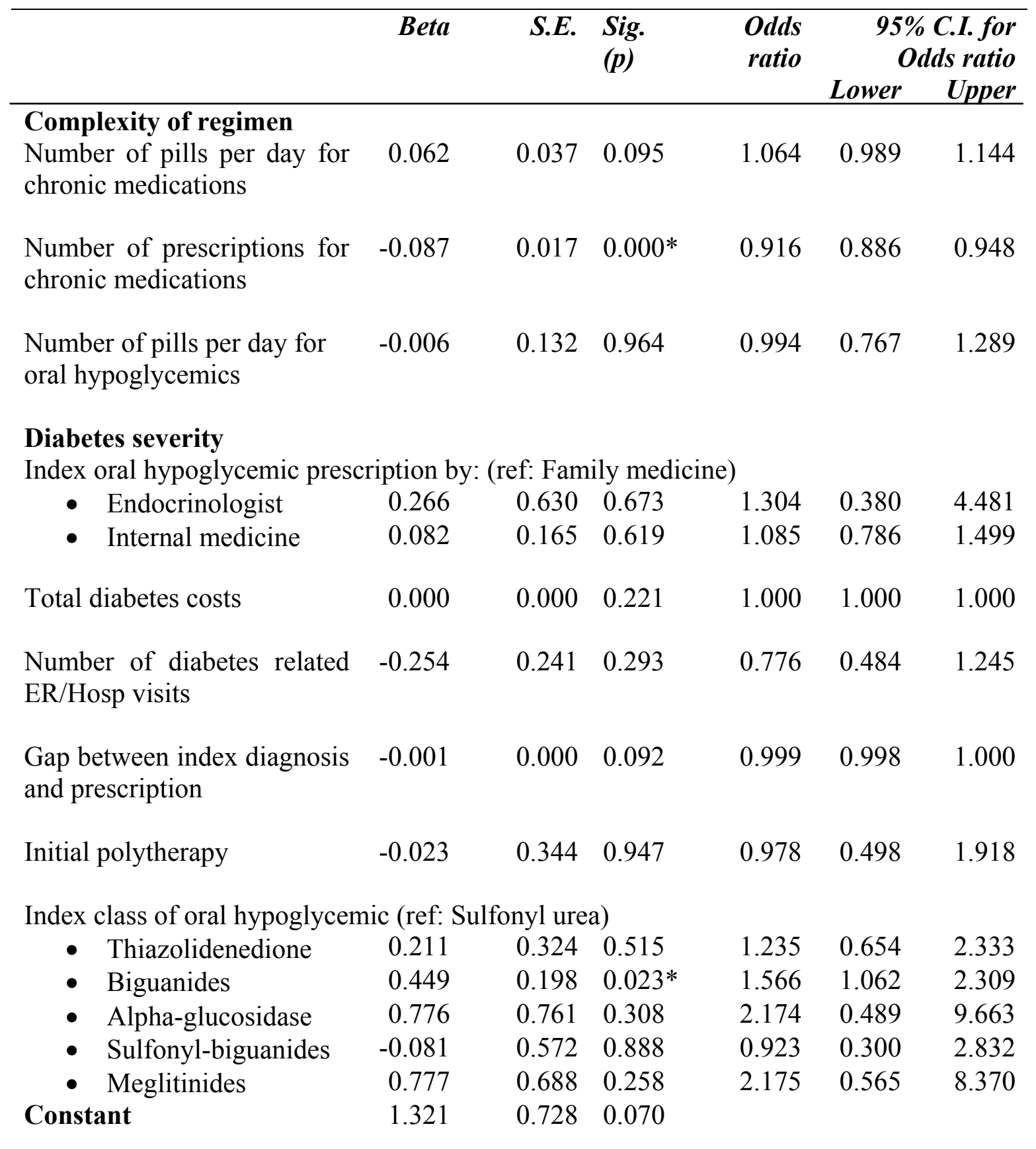


Table 17B: Multinomial Logistic regression for the impact of depression on adherence (Reg MPR-2) to oral hypoglycemics

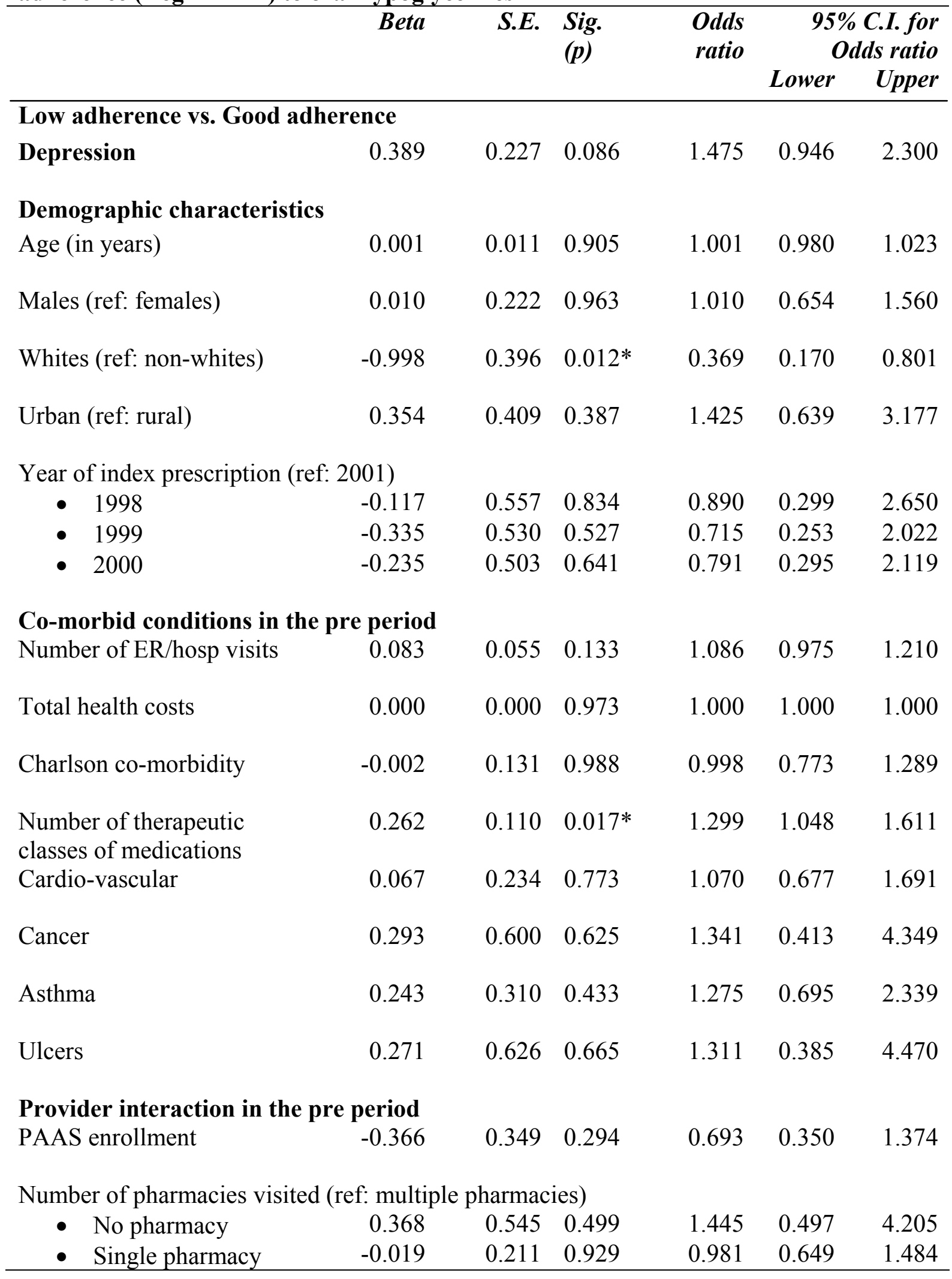


Table 17B: Multinomial Logistic regression for the impact of depression on adherence (Reg MPR-2) to oral hypoglycemics (Contd.)

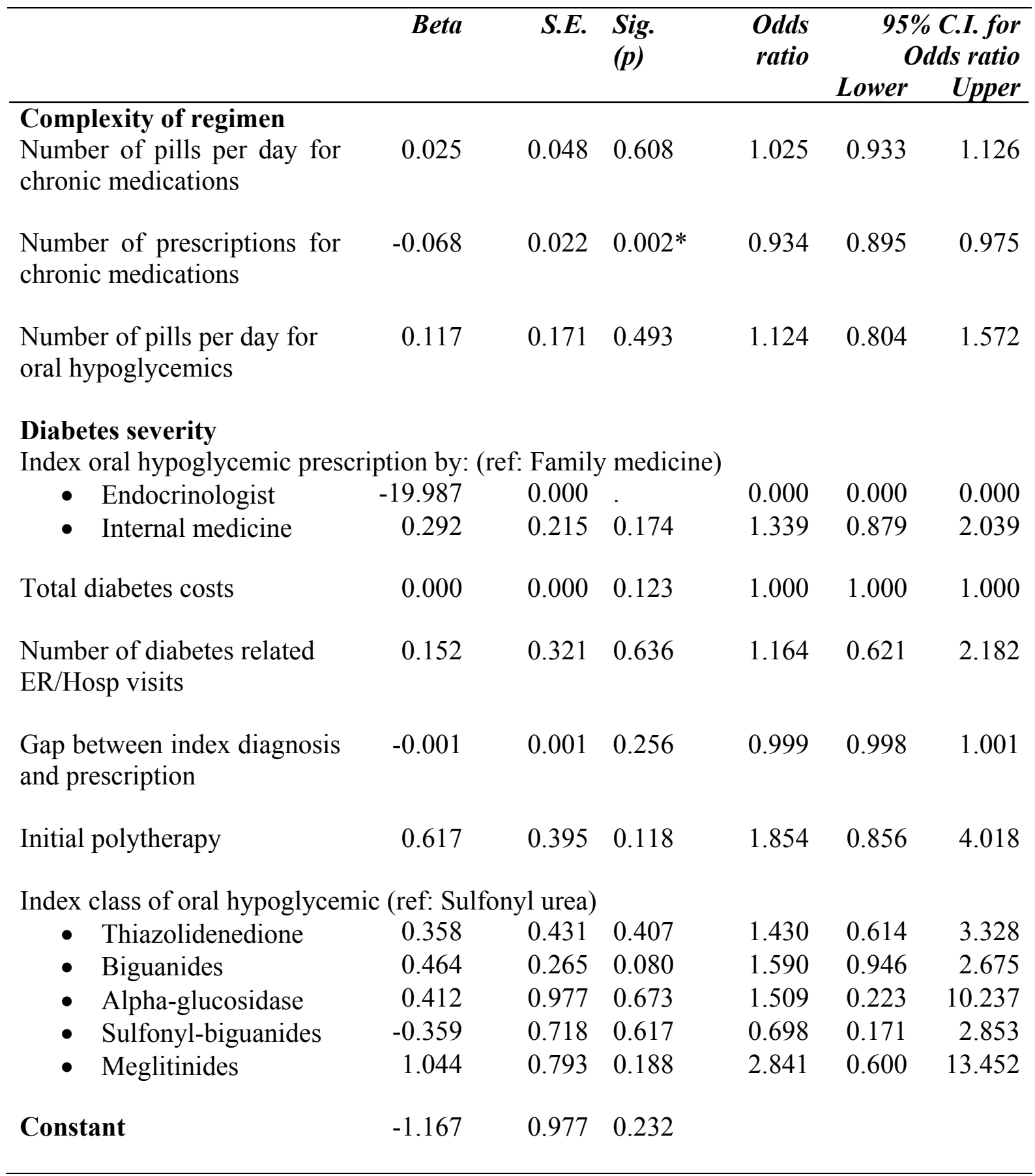


Table 17C: Multinomial Logistic regression for the impact of depression on adherence (Reg MPR-2) to oral hypoglycemics

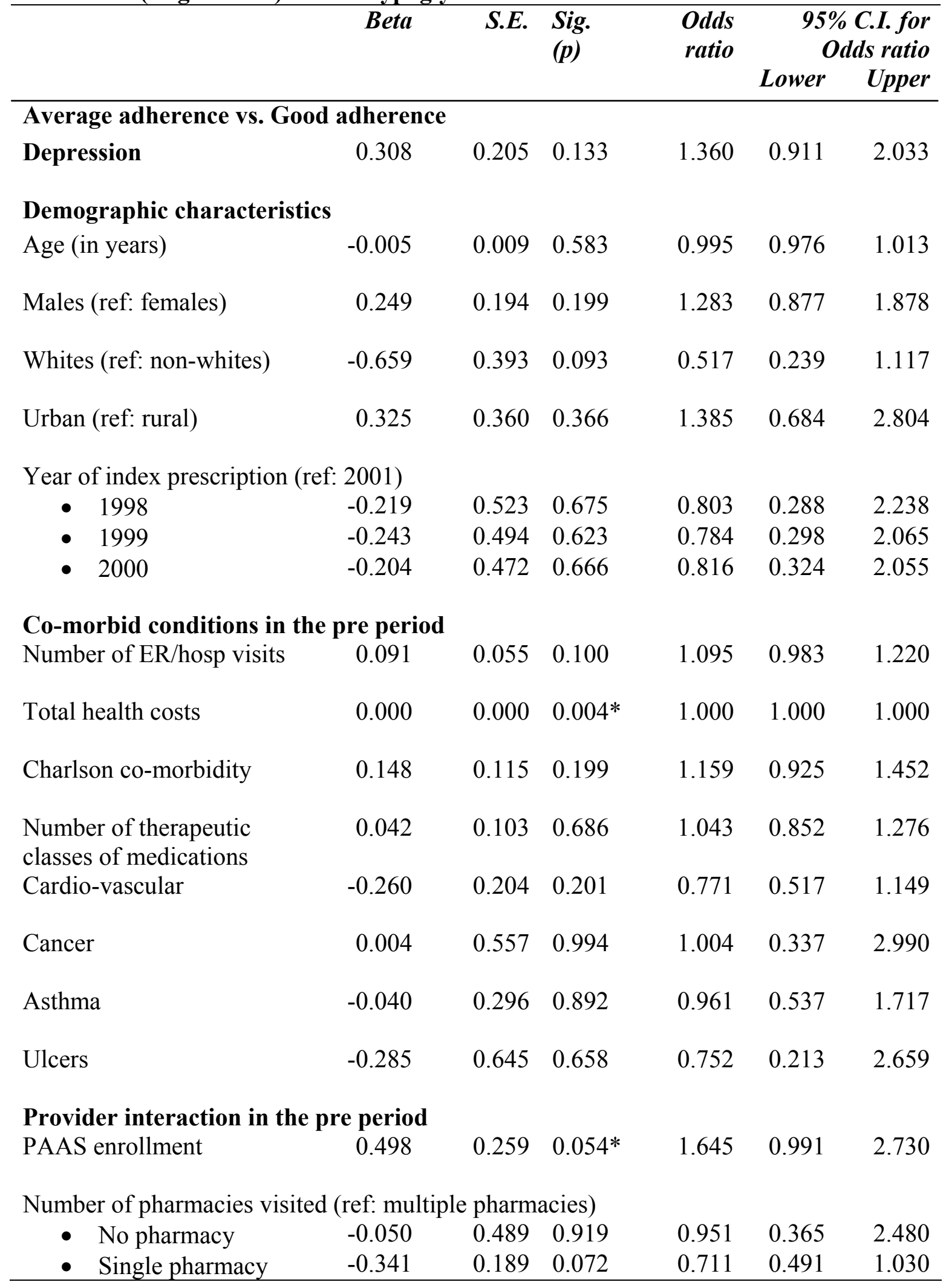


Table 17C: Multinomial Logistic regression for the impact of depression on adherence (Reg MPR-2) to oral hypoglycemics (Contd.)

\begin{tabular}{|c|c|c|c|c|c|c|}
\hline & \multirow[t]{2}{*}{ Beta } & \multirow[t]{2}{*}{ S.E. } & \multirow[t]{2}{*}{$\begin{array}{l}\text { Sig. } \\
(p)\end{array}$} & \multirow[t]{2}{*}{$\begin{array}{c}\text { Odds } \\
\text { ratio }\end{array}$} & \multicolumn{2}{|c|}{$\begin{array}{r}\text { 95\% C.I. for } \\
\text { Odds ratio }\end{array}$} \\
\hline & & & & & Lower & Upper \\
\hline \multicolumn{7}{|l|}{ Complexity of regimen } \\
\hline $\begin{array}{l}\text { Number of pills per day for } \\
\text { chronic medications }\end{array}$ & 0.074 & 0.042 & 0.082 & 1.076 & 0.991 & 1.169 \\
\hline $\begin{array}{l}\text { Number of prescriptions for } \\
\text { chronic medications }\end{array}$ & -0.032 & 0.020 & 0.107 & 0.969 & 0.933 & 1.007 \\
\hline $\begin{array}{l}\text { Number of pills per day for } \\
\text { oral hypoglycemics }\end{array}$ & -0.152 & 0.169 & 0.367 & 0.859 & 0.616 & 1.196 \\
\hline \multicolumn{7}{|l|}{ Diabetes severity } \\
\hline \multicolumn{7}{|c|}{ Index oral hypoglycemic prescription by: (ref: Family medicine) } \\
\hline - Endocrinologist & 0.636 & 0.690 & 0.356 & 1.889 & 0.489 & 7.306 \\
\hline - Internal medicine & 0.015 & 0.204 & 0.940 & 1.016 & 0.681 & 1.515 \\
\hline Total diabetes costs & 0.000 & 0.000 & 0.860 & 1.000 & 1.000 & 1.000 \\
\hline $\begin{array}{l}\text { Number of diabetes related } \\
\text { ER/Hosp visits }\end{array}$ & 0.137 & 0.274 & 0.618 & 1.146 & 0.670 & 1.961 \\
\hline $\begin{array}{l}\text { Gap between index diagnosis } \\
\text { and prescription }\end{array}$ & -0.001 & 0.001 & 0.086 & 0.999 & 0.998 & 1.000 \\
\hline Initial polytherapy & 0.119 & 0.416 & 0.775 & 1.126 & 0.498 & 2.543 \\
\hline \multicolumn{7}{|c|}{ Index class of oral hypoglycemic (ref: Sulfonyl urea) } \\
\hline - Thiazolidenedione & 0.334 & 0.396 & 0.399 & 1.397 & 0.642 & 3.037 \\
\hline - Biguanides & 0.510 & 0.245 & $0.038^{*}$ & 1.666 & 1.030 & 2.695 \\
\hline - Alpha-glucosidase & 1.675 & 0.789 & $0.034 *$ & 5.338 & 1.136 & 25.077 \\
\hline - Sulfonyl-biguanides & 0.181 & 0.694 & 0.794 & 1.199 & 0.308 & 4.669 \\
\hline - Meglitinides & 1.244 & 0.777 & 0.109 & 3.469 & 0.757 & 15.901 \\
\hline Constant & 0.058 & 0.906 & 0.949 & & & \\
\hline
\end{tabular}


Table 17D: Multinomial Logistic regression for the impact of depression on adherence (Reg MPR-2) to oral hypoglycemics

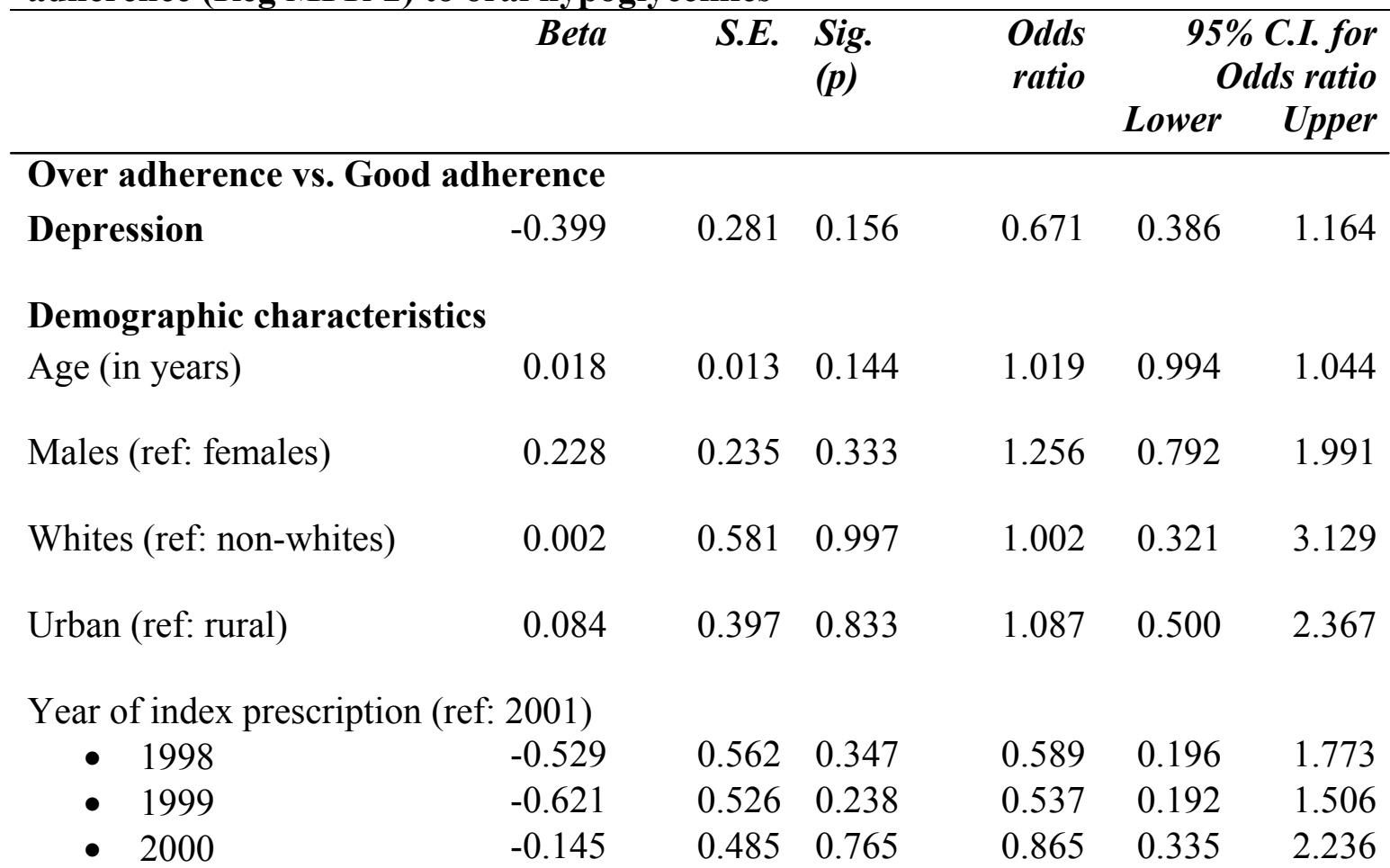

Co-morbid conditions in the pre period

\begin{tabular}{|c|c|c|c|c|c|c|}
\hline Number of ER/hosp visits & 0.137 & 0.054 & $0.011^{*}$ & 1.147 & 1.032 & 1.274 \\
\hline Total health costs & 0.000 & 0.000 & 0.565 & 1.000 & 1.000 & 1.000 \\
\hline Charlson co-morbidity & 0.002 & 0.127 & 0.986 & 1.002 & 0.781 & 1.286 \\
\hline $\begin{array}{l}\text { Number of therapeutic } \\
\text { classes of medications }\end{array}$ & -0.053 & 0.126 & 0.678 & 0.949 & 0.741 & 1.216 \\
\hline Cardio-vascular & -0.284 & 0.260 & 0.275 & 0.753 & 0.452 & 1.253 \\
\hline Cancer & 0.515 & 0.621 & 0.407 & 1.673 & 0.495 & 5.653 \\
\hline Asthma & -0.277 & 0.368 & 0.452 & 0.758 & 0.369 & 1.559 \\
\hline Ulcers & 0.550 & 0.717 & 0.443 & 1.733 & 0.425 & 7.063 \\
\hline \multicolumn{7}{|c|}{ Provider interaction in the pre period } \\
\hline PAAS enrollment & -0.091 & 0.393 & 0.816 & 0.913 & 0.422 & 1.973 \\
\hline \multicolumn{7}{|c|}{ Number of pharmacies visited (ref: multiple pharmacies) } \\
\hline - No pharmacy & 0.514 & 0.589 & 0.382 & 1.673 & 0.528 & 5.302 \\
\hline - Single pharmacy & 0.208 & 0.237 & 0.380 & 1.231 & 0.774 & 1.960 \\
\hline
\end{tabular}


Table 17D: Multinomial Logistic regression for the impact of depression on adherence (Reg MPR-2) to oral hypoglycemics (Contd.)

\begin{tabular}{|c|c|c|c|c|c|c|}
\hline & \multirow[t]{2}{*}{ Beta } & \multirow[t]{2}{*}{ S.E. } & \multirow[t]{2}{*}{$\begin{array}{l}\text { Sig. } \\
(p)\end{array}$} & \multirow[t]{2}{*}{$\begin{array}{l}\text { Odds } \\
\text { ratio }\end{array}$} & \multicolumn{2}{|c|}{$\begin{array}{l}95 \% \text { C.I. for } \\
\text { Odds ratio }\end{array}$} \\
\hline & & & & & Lower & Upper \\
\hline $\begin{array}{l}\text { Complexity of regimen } \\
\text { Number of pills per day for } \\
\text { chronic medications }\end{array}$ & 0.005 & 0.049 & 0.913 & 1.005 & 0.912 & 1.108 \\
\hline $\begin{array}{l}\text { Number of prescriptions for } \\
\text { chronic medications }\end{array}$ & 0.011 & 0.022 & 0.602 & 1.011 & 0.969 & 1.056 \\
\hline $\begin{array}{l}\text { Number of pills per day for } \\
\text { oral hypoglycemics }\end{array}$ & -0.262 & 0.226 & 0.246 & 0.769 & 0.494 & 1.198 \\
\hline \multicolumn{7}{|l|}{ Diabetes severity } \\
\hline $\begin{array}{l}\text { - Endocrinologist } \\
\text { - Internal medicine }\end{array}$ & $\begin{array}{l}1.075 \\
0.282\end{array}$ & $\begin{array}{l}0.765 \\
0.240\end{array}$ & $\begin{array}{l}0.160 \\
0.241\end{array}$ & $\begin{array}{l}2.929 \\
1.326\end{array}$ & $\begin{array}{l}0.654 \\
0.827\end{array}$ & $\begin{array}{r}13.129 \\
2.124\end{array}$ \\
\hline Total diabetes costs & 0.000 & 0.000 & 0.435 & 1.000 & 1.000 & 1.000 \\
\hline $\begin{array}{l}\text { Number of diabetes related } \\
\text { ER/Hosp visits }\end{array}$ & -0.306 & 0.379 & 0.420 & 0.736 & 0.350 & 1.548 \\
\hline $\begin{array}{l}\text { Gap between index diagnosis } \\
\text { and prescription }\end{array}$ & 0.000 & 0.001 & 0.983 & 1.000 & 0.999 & 1.001 \\
\hline Initial polytherapy & 0.172 & 0.462 & 0.709 & 1.188 & 0.480 & 2.939 \\
\hline \multicolumn{7}{|c|}{ Index class of oral hypoglycemic (ref: Sulfonyl urea) } \\
\hline - Thiazolidenedione & 0.531 & 0.416 & 0.201 & 1.701 & 0.753 & 3.843 \\
\hline - Biguanides & 0.210 & 0.314 & 0.503 & 1.234 & 0.667 & 2.286 \\
\hline - Alpha-glucosidase & 1.816 & 0.907 & $0.045^{*}$ & 6.147 & 1.038 & 36.393 \\
\hline - Sulfonyl-biguanides & -0.607 & 0.906 & 0.503 & 0.545 & 0.092 & 3.215 \\
\hline - Meglitinides & 0.740 & 1.202 & 0.538 & 2.095 & 0.199 & 22.099 \\
\hline Constant & -2.113 & 1.128 & 0.061 & & & \\
\hline
\end{tabular}

*Significance at the 0.05 level

PAAS: Physician Assured Access System

Model fit statistics:

Pseudo R-square $=20.0 \%$

-2 Log Likelihood $=3480.436 ; \chi^{2}=270.066 ; p=0.000$ 


\section{Discussion for objective 6}

The study results indicated that newly diagnosed patients who continued oral hypoglycemic therapy had an adherence rate of $88.00 \%$. If patients who discontinued therapy were assumed to be non-adherent and included in the computation of adherence, rates of around $73.00 \%$ were found in the first year of oral hypoglycemic therapy. Results for adherence rates in the 12-month follow up period were found to be comparable to studies in the literature computing adherence rates for newly diagnosed patients using prescription claims data. In a study conducted by Spoelstra and associates ${ }^{161}$ using retrospective claims data of newly diagnosed type 2 diabetes patients, adherence rates of around $86.00 \%$ were found. These numbers are very similar to the ones obtained in this study. An adherence rate of $86.00 \%$ was also found in a study conducted in seniors in Nova Scotia, Canada. ${ }^{164}$ In more than 52,000 patients on sulfonylurea, Boccuzzi and associates ${ }^{16}$ determined an average adherence rate of approximately $79.00 \%$. Similar estimates of $70-80 \%$ were found for newly diagnosed type 2 diabetes patients in Tayside, Scotland. ${ }^{17}$ Slight differences in the reported adherence rates may reflect differences in methods, length of follow-up periods, inclusion criteria, and demographic distribution of the patient population.

Results of this study indicated that patients with depression had significantly lower adherence $($ Reg MPR-1 $=86.00 \%$; Reg MPR-2 $=66.00 \%$ ) to oral hypoglycemics as compared to patients without depression (Reg MPR-1 $=89.00 \%$; Reg MPR-2 $=$ 73.00\%). The impact of depression on adherence remained significant in the multivariate framework. However, the magnitude of difference in adherence was not large. In the only other study examining the impact of depression on adherence with oral 
hypoglycemics, Ciechanowski and colleagues ${ }^{28}$ found that depressive symptom severity was significantly associated with decreased adherence to dietary recommendations and approximately twice as many interruptions in refills of oral hypoglycemics. If these results are converted to make them comparable to findings from this study, rates of $93.00 \%$ adherence were found for patients with low depressive symptomatology as compared to adherence rates of $85.00 \%$ for patients with high depression severity. If patients with medium and high depression severity levels from this study are combined to form a group of depressed patients, adherence rates of $88 \%$ are estimated for depressed patients. Thus, the difference in adherence rates between depressed and non-depressed patients in the study by Ciechanowski and associates was around 5.00\%; similar to the results obtained from our study. However, this study was not restricted to patients who were newly diagnosed patients and included a mix of type 1 and type 2 diabetes patients. Also, the study measured adherence with oral hypoglycemics over a 12-month period, including a period of six months before the assessment of depressive symptoms. This study along with others in the literature demonstrating significantly lower adherence in depressed patients were co-relational in nature and failed to determine whether depression causes non-adherence or non-adherence causes depression. The estimation models could be biased due to endogeneity and failed to control for a number of confounding variables.

In addition to the study by Ciechanowski and associates ${ }^{28}$ in diabetes patients, depression has been reported to have an effect on adherence with other medications across a number of other disease conditions. In a study of adherence with aspirin among elderly patients with coronary heart disease, depressed patients adhered to their regimen 
on a significantly lower proportion of days (45.00\%) than those without depression $(69.00 \%) .{ }^{112}$ Studies of adherence in a variety of other conditions including AIDS, renal failure, and asthma have also identified depression as a risk factor for non-adherence. In a study using prescription refill information from claims data of a large HMO and Veterans Affairs (VA) medical center, depression was significantly associated with adherence of antihypertensive medications. ${ }^{165}$ However, similar to this study's results, the magnitude of decrease in adherence with every unit increase in depression severity was low.

The strength of this study lies in the use of actual prescription refill information from claims data rather than patient reported adherence. Using such an objective measure of adherence was especially important in this study as a pessimistic attitude in depressed patients can cause under-reporting of drug use among these patients and thus show an artificial association between depression and adherence. In addition, data were adjusted for a range of baseline variables such as demographics, co-morbidity, diabetes severity, complexity of regimen, and interaction with health care providers that could confound the impact of depression on adherence with oral hypoglycemics. The study design was also able to avoid the issue of endogeneity by examining adherence in newly diagnosed type 2 diabetes patients who had pre-existing depression. 


\section{Results for Objective 7}

To estimate the impact of preexisting depression on type 2 diabetes related utilization and costs

Claims including either a primary or secondary diagnosis code for type 2 diabetes and prescriptions for insulin, oral hypoglycemics and diabetic supplies were identified in assessing total resource utilization associated with type 2 diabetes. In addition to total type 2 diabetes costs, separate costs in terms of ER/hospitalization costs, outpatient costs, and pharmacy costs were also assessed. Along with expenditures in terms of dollar amounts, utilization related to type 2 diabetes such as number of ER/hospitalization episodes, number of physician office visits, and number of diabetes prescriptions were also compared between depressed and non-depressed patients.

\section{Type 2 diabetes related costs}

Univariate results for type 2 diabetes related costs are reported in Table 18. Results indicated that in the 12 month follow up period total type 2 diabetes related costs were significantly higher for depressed patients $(\$ 3,290.85 \pm 7,060.05)$ as compared to non-depressed patients $(\$ 2,186.24 \pm 5,142.13)$. This difference in total type 2 diabetes costs was primarily due to difference in ER/hospitalization costs, with depressed patients incurring nearly twice the amount of ER/hospitalization costs as non-depressed patients. Type 2 diabetes related outpatient costs were also slightly higher for depressed patients (\$507.42 \pm 678.48$)$ as compared to non-depressed patients ( $\$ 407.82 \pm 629.57)$. However, no differences in diabetes prescription costs were observed. However, these effects could also be a result of confounders such as demographics, co-morbidity, diabetes severity, and interaction with health care providers. Hence, multivariate econometric models were 
used to determine the impact of depression on type 2 diabetes related expenditures controlling for baseline confounding factors.

Semi-log Ordinary Least Squares (OLS) regression techniques were used for the estimation of the impact of preexisting depression on total costs associated with type 2 diabetes controlling for other confounding factors. The semi-log OLS model was found to be significant with a R-square value of $15.60 \%$. The model results are summarized in Table 19. Results of the multivariate model indicated that controlling for confounding covariates, patients with depression incurred $21.30 \%$ higher type 2 diabetes costs as compared to non-depressed patients in the 12 month follow up period $(\mathrm{p}=0.013)$. These estimates were computed by applying the correction of Halverson and Palmquist with a modification by Kennedy. This method was used for interpreting the estimates in all semi-log OLS models. Retransformation using smearing estimated that an excess of $\$ 451.27$ in type 2 diabetes costs were attributable to depression.

In models where the dependent variables are specific costs such as those due to ER/hospitalization visits and outpatient costs, a two-part model was used to estimate the impact of depression on diabetes related ER/hospitalization and outpatient costs. Results for the two-part model estimating the impact of depression on ER/hospitalization costs are summarized in Table 20A-20B. An initial multivariate logistic regression model to estimate the impact of depression on a type 2 diabetes related ER/hospitalization episode demonstrated that depressed patients were nearly 1.4 times more likely to have a ER/hospitalization episode as compared to non-depressed patients ( $\mathrm{p}=0.028)$. A subsequent semi-logarithmic OLS model, which was conducted only on those patients 
incurring type 2 diabetes related ER/hospitalization revealed no significant differences in ER/hospitalization costs between depressed and non-depressed patients.

Two-part models for type 2 diabetes related outpatient costs revealed no significant differences in either the probability or expenditures for type 2 diabetes related outpatient visits between patients with and without depression (Tables 21A-21B). A semi-log OLS model in a multivariate framework also failed to reveal any significant differences in prescription costs between depressed and non-depressed patient controlling for baseline covariates (Table 22).

\section{Type 2 diabetes related utilization}

Univariate results for type 2 diabetes related utilization in the 12-month follow up period are presented in Table 18. The most notable difference between the 2 groups was in the number of ER/Hospitalization episodes, with depressed patients $(0.79 \pm 1.57)$ having significantly higher ER/Hospitalization episodes as compared to non-depressed patients $(0.40 \pm 1.09)$. Patients with depression $(9.81 \pm 5.49)$ had significantly less number of prescriptions for diabetes medications as compared to non-depressed patients (10.76 \pm 6.01$)$, primarily due to decreased adherence with medications. However, the results did not indicate any differences in number of physician office visits between depressed and non-depressed patient.

As the dependent variable in this case is count data, a Poisson or Negative binomial model could be used to estimate the impact of depression on type 2 diabetes related utilization in a multivariate framework. In a Poisson regression, the probability of a count is determined by a Poisson distribution, which has the defining characteristic that the conditional mean of the outcome is equal to the conditional variance. However, most 
of the models for utilization were overdispersed (i.e the variance being greater than the mean) with a high proportion of zero counts. Hence, a negative binomial model was used for estimation of all the utilization related variables.

Results of the negative binomial models indicated that the only significant difference between the two groups in the multivariate framework was in terms of number of type 2 diabetes related ER/Hospitalizations with depressed patients incurring $57.78 \%$ higher number of visits as compared to non depressed patients $(p=0.000)$. Results of this negative binomial model and the fit statistics are summarized in Table 23. Table 24 reports the results of the negative binomial model, which demonstrates no significant impact of depression on number of diabetes related physician office visits. The multivariate framework also failed to reveal any significant impact of depression on number of diabetes related prescriptions in the 12-month follow up period (Table 25). 
Table 18: Utilization and expenditures for type 2 diabetes in the 12-month follow up period

\begin{tabular}{cccc} 
Depressed & Non-depressed & Mann-Whitney & Sig. \\
$($ Mean + S.D. $)$ & $($ Mean + S.D. $)$ & Utest & $(p)$ \\
$(N=471)$ & $(N=855)$ & & \\
\hline
\end{tabular}

Type 2 diabetes related costs

Outpatient costs

507.42

$+678.48$

407.82

$\pm 629.57$

ER/Hosp costs

$$
2,276.38
$$

$+6,932.74$

$1,271.08$

$+4,939.85$

Pharmacy costs

507.05

$+436.31$

507.33

$+475.01$

Total costs

$$
\begin{array}{r}
3,290.85 \\
+7,060.05
\end{array}
$$

$$
2,186.24
$$

$+5,142.13$

$184897.00 \quad 0.014^{*}$

$174006.50 \quad 0.000^{*}$

Type 2 diabetes related utilization

Number of physician

$2.76 \pm 3.34$

$2.55+3.08$

197218.50

0.525

office visits

Number of ER/hosp

$0.79+1.57$

$0.40 \pm 1.09$

172425.50

$0.000 *$

visits

Number of prescriptions $9.81 \pm 5.49$

$10.76 \pm 6.01$

182867.0

$0.005^{*}$

* significance at the 0.05 level 
Table 19: Semi-log Ordinary Least Squares (OLS) regression model for the impact of depression on total diabetes costs

\section{Beta S.E. Test statistic Sig.}

(t)

(p)

\section{Depression}

0.196

0.079

$2.4920 .013^{*}$

Demographic characteristics

Age (in years)

$0.005 \quad 0.004$

$1.419 \quad 0.156$

Males (ref: females)

0.048

0.075

$0.633 \quad 0.527$

Whites (ref: non-whites)

0.078

0.155

$0.505 \quad 0.614$

Urban (ref: rural)

$\begin{array}{llll}-0.184 & 0.129 & -1.431 & 0.153\end{array}$

Year of index prescription (ref: 2001)

- 1998

0.044

0.187

$0.233 \quad 0.815$

- 1999

0.478

0.177

$2.7050 .007 *$

- 2000

0.340

0.166

$2.045 \quad 0.041 *$

Co-morbid conditions in the pre period

Number of ER/hosp visits

$\begin{array}{llll}0.050 & 0.014 & 3.509 & 0.000 *\end{array}$

Total health costs

0.000

0.000

$-1.370 \quad 0.171$

Charlson co-morbidity

$0.123 \quad 0.042$

$2.9470 .003 *$

Number of therapeutic classes

0.077

0.022

$3.4430 .001^{*}$

of medications

Cardio-vascular

$$
0.074 \quad 0.079
$$

$0.935 \quad 0.350$

Cancer

$-0.409$

0.197

$-2.0740 .038 *$

Asthma

$-0.017$

0.106

$-0.163 \quad 0.871$

Ulcers

0.170

0.212

$\begin{array}{ll}0.801 & 0.423\end{array}$

Provider interaction in the pre period

PAAS enrollment
0.125
0.109
$1.146 \quad 0.252$

Number of pharmacies visited (ref: multiple pharmacies)
- No pharmacy
$-0.035$
0.179
$-0.193 \quad 0.847$
- Single pharmacy
$-0.001$
0.073
$-0.020 \quad 0.984$ 
Table 19: Semi-log Ordinary Least Squares (OLS) regression model for the impact of depression on total diabetes costs (Contd.)

\begin{tabular}{|c|c|c|c|c|}
\hline & Beta & S.E. & $\begin{array}{c}\text { Test statistic } \\
(t)\end{array}$ & $\begin{array}{l}\text { Sig. } \\
\text { (p) }\end{array}$ \\
\hline \multicolumn{4}{|l|}{ Diabetes severity } & Index oral hypoglycemic prescription by: (ref: Family medicine) \\
\hline - Endocrinologist & 0.290 & 0.283 & 1.026 & 0.305 \\
\hline - Internal medicine & 0.061 & 0.076 & 0.803 & 0.422 \\
\hline Total diabetes costs & 0.000 & 0.000 & 0.608 & 0.543 \\
\hline $\begin{array}{l}\text { Number of diabetes related } \\
\text { ER/hosp visits }\end{array}$ & 0.261 & 0.103 & 2.524 & $0.012 *$ \\
\hline $\begin{array}{l}\text { Gap between index diagnosis } \\
\text { and prescription }\end{array}$ & 0.000 & 0.000 & 1.605 & 0.109 \\
\hline Initial polytherapy & 0.696 & 0.155 & 4.486 & $0.000 *$ \\
\hline \multicolumn{5}{|c|}{ Index class of oral hypoglycemic (ref: Sulfonyl urea) } \\
\hline - Thiazolidenedione & 0.611 & 0.149 & 4.108 & $0.000^{*}$ \\
\hline - Biguanides & 0.150 & 0.077 & 1.940 & $0.053 *$ \\
\hline - Alpha-glucosidase & -0.075 & 0.284 & -0.264 & 0.792 \\
\hline - Sulfonyl-biguanides & -0.758 & 0.265 & -2.863 & $0.004 *$ \\
\hline - Meglitinides & 0.464 & 0.277 & 1.672 & 0.095 \\
\hline Constant & 5.781 & 0.326 & 17.728 & 0.000 \\
\hline
\end{tabular}

*significance at the 0.05 level

PAAS: Physician Assured Access System

\section{$\underline{\text { Model fit statistics }}$}

R-square: $15.6 \% ; \mathrm{F}=8.331 ; \mathrm{P}=0.000$

Durbin-Watson: 1.988

White test for heteroskedasticity: Chi-square $=409.1604 ; \mathrm{P}=0.1625$ 
TWO-PART MODEL-PART1

Table 20A: Logistic regression for the impact of depression on having a diabetes related ER/hospitalization episode in the 12-month follow-up period

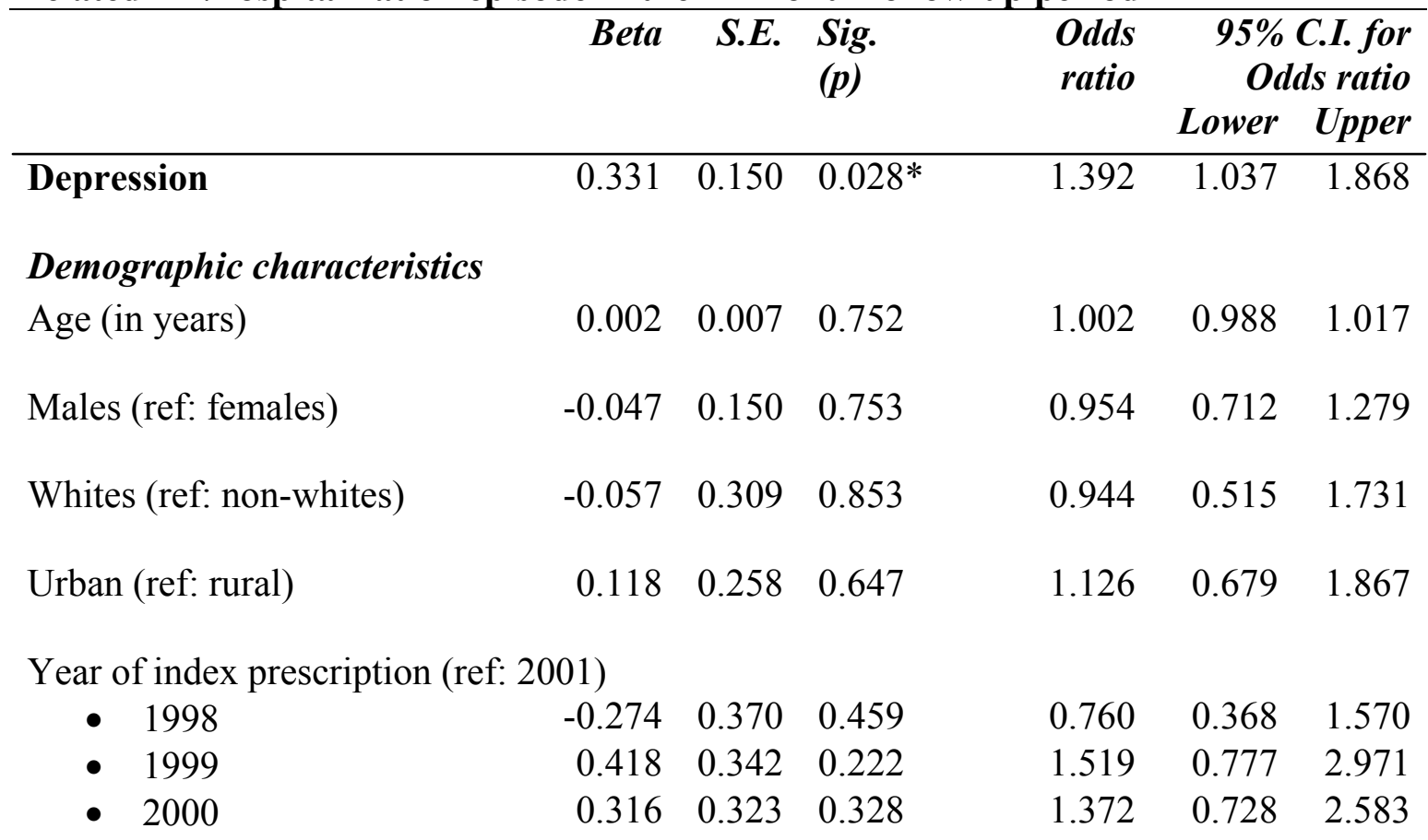

Co-morbid conditions in the pre period

Number of ER/hosp visits

0.230

$0.0380 .000^{*}$

1.259

$1.169 \quad 1.355$

Total health costs

$\begin{array}{lll}0.000 & 0.000 & 0.510\end{array}$

$\begin{array}{lll}1.000 & 1.000 \quad 1.000\end{array}$

Charlson co-morbidity

$\begin{array}{lll}0.139 & 0.077 & 0.071\end{array}$

$1.149 \quad 0.988$

1.337

Number of therapeutic classes of medications

Cardio-vascular

$0.106 \quad 0.042 \quad 0.012 *$

$\begin{array}{lll}1.111 & 1.023 \quad 1.207\end{array}$

Cancer

$\begin{array}{lll}-0.017 & 0.156 & 0.916\end{array}$

0.984

$0.724 \quad 1.336$

$$
\begin{array}{lll}
-0.474 & 0.385 & 0.217
\end{array}
$$

0.622

$0.293 \quad 1.323$

Asthma

$\begin{array}{lll}0.242 & 0.193 & 0.209\end{array}$

1.274

$0.873 \quad 1.858$

Ulcers

$$
\begin{array}{lll}
0.673 & 0.392 & 0.085
\end{array}
$$

1.961

$0.910 \quad 4.224$

Provider interaction in the pre period
PAAS enrollment
$-0.065 \quad 0.215$
0.761
0.937
$0.614 \quad 1.428$

Number of pharmacies visited (ref: multiple pharmacies)

\begin{tabular}{lllllll} 
- No pharmacy & 0.193 & 0.375 & 0.606 & 1.213 & 0.582 & 2.529 \\
- Single pharmacy & 0.065 & 0.144 & 0.649 & 1.068 & 0.806 & 1.415 \\
\hline
\end{tabular}


Table 20A: Logistic regression for the impact of depression on having a diabetes related ER/Hosp episode the 12-month follow-up period (Contd.)

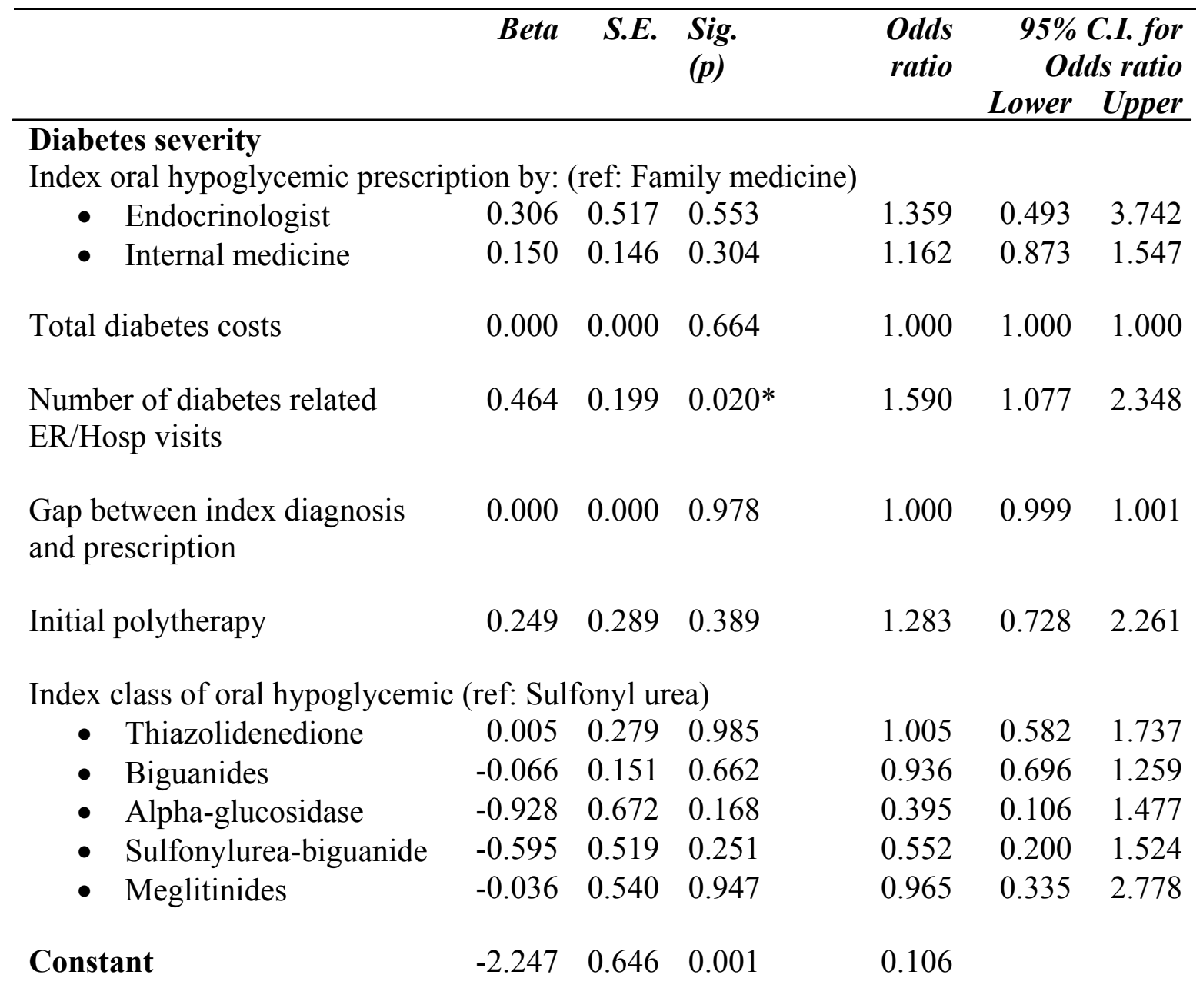

* Significance at the 0.05 level

PAAS: Physician Assured Access System

\section{Model fit statistics:}

Pseudo R-square $=19.6 \%$

-2 Log Likelihood $=1350.585 ; \chi^{2}=189.547, p=0.000$

Hosmer and Lemeshow's goodness of fit: $\chi^{2}=4.653 ; p=0.794$ 
TWO-PART MODEL-PART 2

Table 20B: Semi-log Ordinary Least Squares (OLS) regression model for the impact of depression on diabetes related ER/hospitalization costs

\section{Beta}

$0.232 \quad 0.209$

$1.109 \quad 0.268$

Demographic characteristics

Age (in years)

$0.024 \quad 0.011$

$2.2770 .023^{*}$

Males (ref: females)

Year of index prescription (ref: 2001)

- 1998

- 1999

- 2000
0.246

0.213

0.025

$-0.618$

0.377

$-1.6380 .102$

0.610

0.599

0.256

0.483

0.440

0.421

$1.155 \quad 0.249$

$0.056 \quad 0.955$

$\begin{array}{ll}1.263 & 0.208\end{array}$

$1.360 \quad 0.175$

$\begin{array}{ll}0.610 & 0.543\end{array}$

Co-morbid conditions in the pre period

Number of ER/hosp visits

$\begin{array}{llll}0.015 & 0.027 & 0.550 & 0.583\end{array}$

Total health costs

$0.000 \quad 0.000$

$0.645 \quad 0.519$

Charlson co-morbidity

$0.017 \quad 0.090$

$0.188 \quad 0.851$

Number of therapeutic classes

$0.123 \quad 0.057$

$2.1410 .033^{*}$

of medications

Cardio-vascular

$0.098 \quad 0.229$

$0.428 \quad 0.669$

Cancer

$-0.064 \quad 0.490$

$-0.131 \quad 0.896$

Asthma

$-0.194 \quad 0.246$

$-0.789 \quad 0.431$

Ulcers

$0.415 \quad 0.429$

$\begin{array}{ll}0.967 & 0.334\end{array}$

Provider interaction in the pre period

PAAS enrollment
0.184
0.295
$0.623 \quad 0.534$

Number of pharmacies visited (ref: multiple pharmacies)
- No pharmacy
0.444
0.560
$0.793 \quad 0.428$
- Single pharmacy
$-0.036 \quad 0.200$
$\begin{array}{ll}-0.178 & 0.859\end{array}$ 
Table 20B: Semi-log Ordinary Least Squares (OLS) regression model for the impact of depression on diabetes related ER/Hospitalization costs (Contd.)

Beta S.E. $\quad$ Test statistic Sig.

( $t)$

(p)

Diabetes severity

Index oral hypoglycemic prescription by: (ref: Family medicine)
- Endocrinologist
$0.035 \quad 0.652$
$0.053 \quad 0.957$
- Internal medicine
$-0.009$
0.200
$-0.044 \quad 0.965$

Total diabetes costs

$0.000 \quad 0.000$

$0.388 \quad 0.698$

Number of diabetes related

0.181

0.217

$0.833 \quad 0.405$

ER/Hosp visits

Gap between index diagnosis

$0.000 \quad 0.001$

$0.792 \quad 0.429$

and prescription

Initial polytherapy

$-0.711$

0.417

$-1.705 \quad 0.089$

Index class of oral hypoglycemic (ref: Sulfonyl urea)

- Thiazolidenedione

0.133

0.373

$\begin{array}{ll}0.358 & 0.721\end{array}$

- Biguanides

0.006

0.211

0.0280 .978

- Alpha-glucosidase

$-2.114$

0.928

$-2.2770 .023 *$

- Sulfonyl-biguanides

0.296

0.771

$\begin{array}{ll}0.384 & 0.701\end{array}$

- Meglitinides

0.478

0.772

$0.619 \quad 0.536$

Constant

5.671

0.887

$6.390 \quad 0.000$

* significance at the 0.05 level

PAAS: Physician Assured Access System

\section{Model fit statistics:}

R-square: $13.5 \% ; \mathrm{F}=1.748 ; \mathrm{P}=0.010$

Durbin-Watson: 2.004

White test for heteroskedasticity: Chi-square $=272.42 ; p=0.7762$ 
TWO-PART MODEL-PART1

Table 21A: Logistic regression for the impact of depression on having a diabetes related outpatient episode

\begin{tabular}{|c|c|c|c|c|c|c|}
\hline & \multirow[t]{2}{*}{ Beta } & \multirow[t]{2}{*}{ S.E. } & \multirow[t]{2}{*}{$\begin{array}{l}\text { Sig. } \\
\text { (p) }\end{array}$} & \multirow[t]{2}{*}{$\begin{array}{c}\text { Odds } \\
\text { ratio }\end{array}$} & \multicolumn{2}{|c|}{$\begin{array}{r}95 \% \text { C.I. for } \\
\text { Odds ratio }\end{array}$} \\
\hline & & & & & Lower & Upper \\
\hline Depression & -0.118 & 0.221 & 0.594 & 0.889 & 0.576 & 1.371 \\
\hline \multicolumn{7}{|c|}{ Demographic characteristics } \\
\hline Age (in years) & -0.008 & 0.010 & 0.402 & 0.992 & 0.972 & 1.011 \\
\hline Males (ref: females) & 0.223 & 0.215 & 0.299 & 1.250 & 0.821 & 1.903 \\
\hline Whites (ref: non-whites) & 0.289 & 0.381 & 0.448 & 1.335 & 0.633 & 2.814 \\
\hline Urban (ref: rural) & -0.181 & 0.382 & 0.635 & 0.834 & 0.395 & 1.763 \\
\hline \multicolumn{7}{|c|}{ Year of index prescription (ref: 2001) } \\
\hline - 1998 & -0.995 & 0.612 & 0.104 & 0.370 & 0.111 & 1.228 \\
\hline - 1999 & -0.013 & 0.598 & 0.983 & 0.987 & 0.306 & 3.188 \\
\hline - 2000 & 0.288 & 0.586 & 0.623 & 1.333 & 0.423 & 4.206 \\
\hline
\end{tabular}

Co-morbid conditions in the pre period

Number of ER/hosp visits

$0.048 \quad 0.349$

$\begin{array}{lll}1.046 & 0.952 \quad 1.148\end{array}$

Total health costs

$\begin{array}{llllll}0.000 & 0.000 & 0.000 * & 1.000 & 1.000 & 1.000\end{array}$

Charlson co-morbidity

0.051

0.155

0.742

$\begin{array}{ll}1.052 & 0.777\end{array}$

1.425

Number of therapeutic classes

of medications

Cardio-vascular

$\begin{array}{lll}-0.078 & 0.061 & 0.203\end{array}$

$0.925 \quad 0.820 \quad 1.043$

Cancer

$0.802 \quad 0.222 \quad 0.000^{*}$

$2.230 \quad 1.444 \quad 3.446$

$\begin{array}{lll}0.910 & 0.832 & 0.274\end{array}$

2.484

$0.487 \quad 12.675$

Asthma

$\begin{array}{lll}-0.028 & 0.329 & 0.933\end{array}$

$0.973 \quad 0.511 \quad 1.852$

Ulcers

$\begin{array}{lll}-0.006 & 0.665 & 0.993\end{array}$

$0.994 \quad 0.270 \quad 3.659$

Provider interaction in the pre period
PAAS enrollment
0.256
$0.325 \quad 0.431$
$1.292 \quad 0.683$
2.443

Number of pharmacies visited (ref: multiple pharmacies)

\begin{tabular}{lllllll} 
- No pharmacy & -0.648 & 0.446 & 0.147 & 0.523 & 0.218 & 1.254 \\
- Single pharmacy & -0.414 & 0.210 & $0.049^{*}$ & 0.661 & 0.438 & 0.997 \\
\hline
\end{tabular}


Table 21A: Logistic regression for the impact of depression on having a diabetes related outpatient episode (Contd.)

$\begin{array}{llllr}\text { Beta } & \text { S.E. } & \begin{array}{l}\text { Sig. } \\ (p)\end{array} & \begin{array}{r}\text { Odds } \\ \text { ratio }\end{array} & \begin{array}{r}95 \% \text { C.I. for } \\ \text { Odd ratio } \\ \text { Lower }\end{array} \\ & & & \text { Upper }\end{array}$

\section{Diabetes severity}

Index oral hypoglycemic prescription by: (ref: Family medicine)

$\begin{array}{lllllrr}\text { - Endocrinologist } & 0.607 & 1.065 & 0.568 & 1.836 & 0.228 & 14.788 \\ \text { - Internal medicine } & 0.334 & 0.230 & 0.147 & 1.396 & 0.890 & 2.192\end{array}$

$\begin{array}{lllllll}\text { Total diabetes costs } & 0.000 & 0.000 & 0.793 & 1.000 & 1.000 & 1.000\end{array}$

$\begin{array}{lllllll}\text { Number of diabetes related } & -0.068 & 0.332 & 0.838 & 0.934 & 0.487 & 1.791\end{array}$

ER/Hosp visits

$\begin{array}{lllllll}\text { Gap between index diagnosis } & 0.001 & 0.001 & 0.504 & 1.001 & 0.999 & 1.002\end{array}$ and prescription
Initial polytherapy
$\begin{array}{lll}1.092 & 0.619 & 0.078\end{array}$
$2.981 \quad 0.886 \quad 10.028$

Index class of oral hypoglycemic (ref: Sulfonyl urea)
- Thiazolidenedione
$\begin{array}{lll}-0.049 & 0.508 & 0.924\end{array}$
$\begin{array}{lll}0.953 & 0.352 & 2.576\end{array}$
- Biguanides
$\begin{array}{lll}-0.013 & 0.221 & 0.954\end{array}$
$\begin{array}{lll}0.987 & 0.640 & 1.524\end{array}$
- Alpha-glucosidase
$\begin{array}{lll}-0.276 & 0.669 & 0.680\end{array}$
$\begin{array}{lll}0.759 & 0.205 & 2.814\end{array}$
- Sulfonylurea-biguanide
$\begin{array}{lll}-0.314 & 1.208 & 0.795\end{array}$
$\begin{array}{lll}0.730 & 0.068 & 7.788\end{array}$
- Meglitinides
$\begin{array}{lll}-0.526 & 0.680 & 0.440\end{array}$
$0.591 \quad 0.156$
2.243
$\begin{array}{lll}2.589 & 0.968 & 0.007\end{array}$
13.320

Constant

*significance at the 0.05 level

PAAS: Physician Assured Access System

\section{Model fit statistics:}

Pseudo R-square $=14.9 \%$

-2 Log Likelihood $=759.410 ; \chi^{2}=97.180, \mathrm{p}=0.000$

Hosmer and Lemeshow's goodness of fit: $\chi^{2}=5.411 ; p=0.713$ 
TWO-PART MODEL-PART 2

Table 21B: Semi-log Ordinary Least Squares (OLS) regression model for the impact of depression on diabetes related outpatient costs

Beta S.E. Test statistic Sig.

( $t)$

$0.065 \quad 0.091$
$0.717 \quad 0.473$

Demographic characteristics

\begin{tabular}{|c|c|c|c|c|}
\hline Age (in years) & -0.007 & 0.004 & -1.519 & 0.129 \\
\hline Males (ref: females) & -0.054 & 0.087 & -0.619 & 0.536 \\
\hline Whites (ref: non-whites) & 0.120 & 0.183 & 0.654 & 0.513 \\
\hline Urban (ref: rural) & -0.176 & 0.148 & -1.190 & 0.234 \\
\hline \multicolumn{5}{|c|}{ Year of index prescription (ref: 2001) } \\
\hline - 1998 & -0.095 & 0.214 & -0.445 & 0.656 \\
\hline - 1999 & 0.056 & 0.199 & 0.279 & 0.780 \\
\hline - 2000 & 0.308 & 0.188 & 1.639 & 0.101 \\
\hline
\end{tabular}

Co-morbid conditions in the pre period

Number of ER/hosp visits

$\begin{array}{llll}0.030 & 0.017 & 1.721 & 0.085\end{array}$

Total health costs

$0.000 \quad 0.000$

$-1.157 \quad 0.247$

Charlson co-morbidity

$0.102 \quad 0.047$

$2.154 \quad 0.031$

Number of therapeutic classes

of medications

Cardio-vascular

$0.000 \quad 0.026$

0.093

0.222

$-0.162 \quad 0.872$

Cancer

$-0.036$

0.005

0.122

$0.043 \quad 0.966$

Ulcers

0.123

0.243

$0.508 \quad 0.611$

Provider interaction in the pre period

PAAS enrollment

0.125

$3.1830 .001^{*}$

Number of pharmacies visited (ref: multiple pharmacies)
- No pharmacy
$-0.329$
0.212
$-1.554 \quad 0.121$
- Single pharmacy
$-0.017$
0.084
$-0.201 \quad 0.841$ 
Table 21B: Semi-log Ordinary Least Squares (OLS) regression model for the impact of depression on diabetes related outpatient costs (Contd.)

\begin{tabular}{|c|c|c|c|c|}
\hline & Beta & S.E. & $\begin{array}{c}\text { Test statistic } \\
(t)\end{array}$ & $\begin{array}{l}\text { Sig. } \\
(p)\end{array}$ \\
\hline \multicolumn{5}{|l|}{ Diabetes severity } \\
\hline \multicolumn{5}{|c|}{ Index oral hypoglycemic prescription by: (ref: Family medicine) } \\
\hline - Endocrinologist & 0.199 & 0.318 & 0.628 & 0.530 \\
\hline - Internal medicine & -0.125 & 0.088 & -1.427 & 0.154 \\
\hline Total diabetes costs & 0.000 & 0.000 & 0.921 & 0.357 \\
\hline \multicolumn{4}{|l|}{ ER/Hosp visits } & 0.908 \\
\hline $\begin{array}{l}\text { Gap between index diagnosis } \\
\text { and prescription }\end{array}$ & 0.000 & 0.000 & 1.823 & 0.069 \\
\hline Initial polytherapy & 0.428 & 0.175 & 2.455 & $0.014^{*}$ \\
\hline \multicolumn{5}{|c|}{ Index class of oral hypoglycemic (ref: Sulfonyl urea) } \\
\hline - Thiazolidenedione & -0.175 & 0.168 & -1.044 & 0.297 \\
\hline - Biguanides & -0.104 & 0.089 & -1.175 & 0.240 \\
\hline - Alpha-glucosidase & -0.072 & 0.336 & -0.215 & 0.830 \\
\hline - Sulfonyl-biguanides & -0.813 & 0.296 & -2.747 & $0.006^{*}$ \\
\hline - Meglitinides & -0.073 & 0.328 & -0.222 & 0.824 \\
\hline Constant & 5.498 & 0.378 & 14.531 & 0.000 \\
\hline
\end{tabular}

*significance at the 0.05 level

PAAS: Physician Assured Access System

\section{Model fit statistics:}

R-square: $7.6 \% ; \mathrm{F}=3.080 ; \mathrm{P}=0.010$

Durbin-Watson: 2.009

White test for heteroskedasticity: $\chi^{2}=328.00 ; p=0.8161$ 
Table 22: Semi-log Ordinary Least Squares (OLS) regression model for the impact of depression on diabetes prescription costs

Beta S.E. Test statistic Sig.

(t)

(p)

Depression

0.067

0.065

$1.033 \quad 0.302$

Demographic characteristics

Age (in years)

$0.005 \quad 0.003$

$1.714 \quad 0.087$

Males (ref: females)

0.040

0.062

$0.649 \quad 0.517$

Whites (ref: non-whites)

0.124

0.127

$0.972 \quad 0.331$

Urban (ref: rural)

0.009

0.106

$0.081 \quad 0.935$

Year of index prescription (ref: 2001)

- 1998

0.315

0.521

0.154

- 1999

0.200

0.145

0.137

- 2000

Co-morbid conditions in the pre period

Number of ER/hosp visits

$-0.030$

0.012

0.000

0.000

0.010

0.034

0.042

0.018

$2.0460 .041^{*}$

$3.586 \quad 0.000 *$

$1.463 \quad 0.144$

Total health costs

Charlson co-morbidity

Number of therapeutic classes

of medications

Cardio-vascular

0.028

0.065

$-0.218$

0.162

$-0.162$

0.088

0.174

$-0.238$

$-1.363$

0.173

Provider interaction in the pre period

PAAS enrollment

$\begin{array}{llll}0.004 & 0.090 & 0.042 & 0.966\end{array}$

Number of pharmacies visited (ref: multiple pharmacies)

- No pharmacy

$-0.057$

0.148

0.058

0.060

$\begin{array}{ll}-0.388 & 0.698\end{array}$

- Single pharmacy

0.058


Table 22: Semi-log Ordinary Least Squares (OLS) regression model for the impact of depression on diabetes prescription costs (Contd.)

Beta S.E. Test statistic Sig.

( $t)$

(p)

Diabetes severity

Index oral hypoglycemic prescription by: (ref: Family medicine)
- Endocrinologist
$0.413 \quad 0.233$
$\begin{array}{ll}1.777 \quad 0.076 \\ 0.742 & 0.458\end{array}$
- Internal medicine
0.047
0.063
$0.742 \quad 0.458$

Total diabetes costs

$0.000 \quad 0.000$

$\begin{array}{ll}0.873 & 0.383\end{array}$

Number of diabetes related

$0.063 \quad 0.085$

$0.746 \quad 0.456$

ER/Hosp visits

Gap between index diagnosis

0.000

0.000

$0.680 \quad 0.497$

and prescription

Initial polytherapy

1.210

0.128

$9.4820 .000^{*}$

Index class of oral hypoglycemic (ref: Sulfonyl urea)

$\begin{array}{lllll}\text { - Thiazolidenedione } & 1.270 & 0.122 & 10.368 & 0.000\end{array}$

$\begin{array}{llllll}\text { - Biguanides } & 0.568 & 0.064 & 8.935 & 0.000^{*}\end{array}$

$\begin{array}{lllll}\text { - Alpha-glucosidase } & 0.544 & 0.233 & 2.330 & 0.020^{*}\end{array}$

$\begin{array}{lllll}\text { - Sulfonyl-biguanides } & -0.552 & 0.218 & -2.534 & 0.011^{*}\end{array}$

$\begin{array}{lllll}\text { - Meglitinides } & 0.893 & 0.228 & 3.913 & 0.000 *\end{array}$

$\begin{array}{lllll}\text { Constant } & 4.527 & 0.268 & 16.868 & 0.000\end{array}$

*significance at the 0.05 level

PAAS: Physician Assured Access System

\section{Model fit statistics:}

R-square: $19.9 \% ; \mathrm{F}=10.450 ; \mathrm{P}=0.00$

Durbin-Watson: 1.939

White test for heteroskedasticity: $\chi^{2}=398.14 ; p=0.0705$ 
Table 23: Negative binomial model for the impact of depression on number of diabetes related ER/Hosp episodes

$\begin{array}{llll}\text { Estimate } & \text { S.E. } & \begin{array}{l}\text { Test statistic } \\ (\text { Chi-square })\end{array} & \begin{array}{c}\text { Sig. } \\ (p)\end{array}\end{array}$
Depression
0.456
0.123
$13.740 \quad 0.000 *$

Demographic characteristics

Age (in years)

$\begin{array}{llll}0.001 & 0.006 & 0.020 & 0.902\end{array}$

Males (ref: females)

$\begin{array}{llll}0.173 & 0.123 & 2.000 & 0.157\end{array}$

Whites (ref: non-whites)

$-0.284 \quad 0.244$

$1.350 \quad 0.245$

Urban (ref: rural)

$\begin{array}{llll}0.146 & 0.225 & 0.420 & 0.517\end{array}$

Year of index prescription (ref: 2001)
- 1998
$-0.090$
0.300
$0.090 \quad 0.764$
- 1999
0.364
0.275
$1.750 \quad 0.186$
- 2000
0.368
0.264
$1.940 \quad 0.164$

Co-morbid conditions in the pre period

Number of ER/hosp visits

$\begin{array}{llll}0.149 & 0.024 & 37.880 & 0.000 *\end{array}$

Total health costs

$0.000 \quad 0.000$

$0.290 \quad 0.589$

Charlson co-morbidity

$0.059 \quad 0.058$

$1.050 \quad 0.306$

Number of therapeutic classes

0.062

0.034

$3.280 \quad 0.070$

of medications

Cardio-vascular

$-0.060$

0.131

$0.210 \quad 0.648$

Cancer

$-0.308$

0.299

$1.060 \quad 0.303$

Asthma

0.192

0.153

$1.580 \quad 0.208$

Ulcers

0.202

0.305

$\begin{array}{ll}0.440 & 0.507\end{array}$

Provider interaction in the pre period

PAAS enrollment

$\begin{array}{llll}-0.095 & 0.175 & 0.290 & 0.587\end{array}$

Number of pharmacies visited (ref: multiple pharmacies)

- No pharmacy

0.081

0.306

$0.070 \quad 0.792$

- Single pharmacy

$0.076 \quad 0.119$

$0.410 \quad 0.524$ 
Table 23: Negative binomial model for the impact of depression on number of diabetes related ER/Hosp episodes (Contd.)

\begin{tabular}{|c|c|c|c|c|}
\hline & Estimate & S.E. & $\begin{array}{l}\text { Test statistic } \\
\text { (Chi-square) }\end{array}$ & $\begin{array}{l}\text { Sig. } \\
\text { (p) }\end{array}$ \\
\hline \multicolumn{5}{|l|}{ Diabetes severity } \\
\hline \multicolumn{5}{|c|}{ Index oral hypoglycemic prescription by: (ref: Family medicine) } \\
\hline - Endocrinologist & 0.457 & 0.392 & 1.360 & 0.243 \\
\hline - Internal medicine & 0.197 & 0.120 & 2.710 & 0.100 \\
\hline Total diabetes costs & 0.000 & 0.000 & 1.060 & 0.303 \\
\hline \multicolumn{5}{|l|}{ ER/Hosp visits } \\
\hline $\begin{array}{l}\text { Gap between index diagnosis } \\
\text { and prescription }\end{array}$ & 0.000 & 0.000 & 0.350 & 0.555 \\
\hline Initial polytherapy & 0.318 & 0.240 & 1.760 & 0.185 \\
\hline \multicolumn{5}{|c|}{ Index class of oral hypoglycemic (ref: Sulfonyl urea) } \\
\hline - Thiazolidenedione & 0.085 & 0.222 & 0.150 & 0.701 \\
\hline - Biguanides & -0.070 & 0.123 & 0.320 & 0.572 \\
\hline - Alpha-glucosidase & -0.758 & 0.533 & 2.020 & 0.155 \\
\hline - Sulfonyl-biguanides & -0.573 & 0.452 & 1.600 & 0.205 \\
\hline - Meglitinides & 0.059 & 0.440 & 0.020 & 0.893 \\
\hline Constant & -1.782 & 0.523 & 11.620 & 0.001 \\
\hline
\end{tabular}

*significance at the 0.05 level

PAAS: Physician Assured Access System

\section{Model fit statistics:}

-2log likelihood $=2281.1894 ; \chi^{2=} 205.37 ; p=0.000$

Dispersion estimate: $1.4288 ; \chi^{2}=236.39 ; p=0.000$ 
Table 24: Negative binomial model for the impact of depression on number of diabetes related physician office visits

\section{Estimate $\quad$ S.E. Test statistic Sig. \\ (Chi-square) (p)}

\section{Depression}

0.063

0.081

$0.610 \quad 0.435$

Demographic characteristics

Age (in years)

$0.008 \quad 0.004$

$4.070 \quad 0.044^{*}$

Males (ref: females)

$-0.042$

0.078

$0.290 \quad 0.592$

Whites (ref: non-whites)

0.195

0.166

$1.380 \quad 0.240$

Urban (ref: rural)

0.037

0.137

$0.070 \quad 0.788$

Year of index prescription (ref: 2001)

- 1999

- 2000
- 1998

$-0.374$

$-0.031$

0.080

0.193

0.180

0.170

Number of ER/hosp visits

Total health costs

Charlson co-morbidity

Number of therapeutic classes

of medications

Cardio-vascular

Cancer

Asthma

Ulcers
0.016

$0.150 \quad 0.694$

0.006

0.000

0.000

$5.420 \quad 0.020 *$

0.031

0.043

$0.520 \quad 0.469$

$-0.020$

0.023

$0.750 \quad 0.385$

$$
0.055
$$

0.085

$0.420 \quad 0.517$

$-0.029$

0.198

$0.020 \quad 0.886$

$-0.030$

0.108

$0.080 \quad 0.782$

0.138

0.211

$0.430 \quad 0.512$

Provider interaction in the pre period

PAAS enrollment
0.055
0.112
$0.240 \quad 0.626$

Number of pharmacies visited (ref: multiple pharmacies)
- No pharmacy
$-0.185$
0.193
$0.920 \quad 0.337$
- Single pharmacy
$-0.013 \quad 0.077$
-130
$0.030 \quad 0.863$ 
Table 24: Negative binomial model for the impact of depression on number of diabetes related physician office visits (Contd.)

\begin{tabular}{|c|c|c|c|c|}
\hline & Estimate & S.E. & $\begin{array}{l}\text { Test statistic } \\
\text { (Chi-square) }\end{array}$ & $\begin{array}{l}\text { Sig. } \\
(p)\end{array}$ \\
\hline \multicolumn{5}{|l|}{ Diabetes severity } \\
\hline \multicolumn{5}{|c|}{ Index oral hypoglycemic prescription by: (ref: Family medicine) } \\
\hline - Endocrinologist & 0.474 & 0.280 & 2.870 & 0.090 \\
\hline - Internal medicine & 0.149 & 0.080 & 3.480 & 0.062 \\
\hline Total diabetes costs & 0.000 & 0.000 & 0.550 & 0.457 \\
\hline $\begin{array}{l}\text { Number of diabetes related } \\
\text { ER/Hosp visits }\end{array}$ & -0.023 & 0.106 & 0.050 & 0.829 \\
\hline $\begin{array}{l}\text { Gap between index diagnosis } \\
\text { and prescription }\end{array}$ & 0.000 & 0.000 & 0.610 & 0.434 \\
\hline Initial polytherapy & 0.337 & 0.156 & 4.660 & $0.031 *$ \\
\hline \multicolumn{5}{|c|}{ Index class of oral hypoglycemic (ref: Sulfonyl urea) } \\
\hline - Thiazolidenedione & 0.295 & 0.150 & 3.900 & $0.048 *$ \\
\hline - Biguanides & 0.074 & 0.080 & 0.840 & 0.358 \\
\hline - Alpha-glucosidase & 0.369 & 0.289 & 1.630 & 0.202 \\
\hline - Sulfonyl-biguanides & -0.217 & 0.268 & 0.650 & 0.419 \\
\hline - Meglitinides & -0.065 & 0.292 & 0.050 & 0.825 \\
\hline Constant & 0.332 & 0.348 & 0.910 & 0.340 \\
\hline
\end{tabular}

*significance at the 0.05 level

PAAS: Physician Assured Access System

\section{Model fit statistics:}

-2loglikelihood $=5437.492 ; \chi^{2}=58.82 ; p=0.0013$

Dispersion estimate: $1.2206 ; \chi^{2}=1510.38 ; p=0.000$ 
Table 25: Negative binomial model for the impact of depression on number of diabetes prescriptions

\section{$\begin{array}{llll}\text { Estimate } & \text { S.E. } & \text { Test statistic } & \text { Sig. }\end{array}$ (Chi-square) (p)}

Depression

Demographic characteristics

Age (in years)

Males (ref: females)

Whites (ref: non-whites)

Urban (ref: rural)

Year of index prescription (ref: 2001)

- 1998

- 1999

- 2000
$-0.059$

0.035

$2.850 \quad 0.091$

$0.004 \quad 0.002$

$4.460 \quad 0.035^{*}$

0.003

0.033

$0.010 \quad 0.940$

$0.111 \quad 0.070$

$2.530 \quad 0.112$

0.044

0.057

$0.580 \quad 0.446$

$\begin{array}{ll}0.300 & 0.584\end{array}$

$0.045-0.083$

$\begin{array}{ll}1.780 & 0.183\end{array}$

0.104

0.078

$\begin{array}{ll}1.370 & 0.241\end{array}$

Co-morbid conditions in the pre period

Number of ER/hosp visits $(0.0$

Total health costs

$0.000 \quad 0.000$

$\begin{array}{ll}-0.023 & 0.019\end{array}$

0.010

$11.490 \quad 0.001^{*}$

Charlson co-morbidity

0.027

0.010

of medications

Cardio-vascular

$0.023 \quad 0.036$

$0.400 \quad 0.528$

Cancer

$-0.144$

0.089

$2.640 \quad 0.104$

Asthma

$-0.078 \quad 0.048$
-0.063

Ulcers

$-0.063$

0.096

$2.670 \quad 0.103$

$0.420 \quad 0.515$

Provider interaction in the pre period

PAAS enrollment

$\begin{array}{llll}0.023 & 0.048 & 0.230 & 0.635\end{array}$

Number of pharmacies visited (ref: multiple pharmacies)

- No pharmacy

$-0.028$

0.080

$\begin{array}{ll}0.120 & 0.727\end{array}$

- Single pharmacy

$0.038 \quad 0.033$

$\begin{array}{ll}1.340 & 0.247\end{array}$ 
Table 25: Negative binomial model for the impact of depression on number of diabetes prescriptions (Contd.)

$\begin{array}{llll}\text { Estimate } & \text { S.E. } & \text { Test statistic Sig. }\end{array}$

(Chi-square) (p)

\section{Diabetes severity}

Index oral hypoglycemic prescription by: (ref: Family medicine)
- Endocrinologist
0.241
0.122
$3.920 \quad 0.048^{*}$
- Internal medicine
0.001
0.034
$0.000 \quad 0.985$

Total diabetes costs

$0.000 \quad 0.000$

$4.060 \quad 0.044^{*}$

Number of diabetes related

$0.011 \quad 0.045$

$0.060 \quad 0.808$

ER/Hosp visits

Gap between index diagnosis

$0.000 \quad 0.000$

$1.580 \quad 0.209$

and prescription

Initial polytherapy

0.672

0.064

$111.710 \quad 0.000^{*}$

Index class of oral hypoglycemic (ref: Sulfonyl urea)

- Thiazolidenedione

0.043

0.065

$0.440 \quad 0.507$

- Biguanides

$-0.065$

0.034

$3.530 \quad 0.060$

- Alpha-glucosidase

0.082

0.125

$0.440 \quad 0.509$

- Sulfonyl-biguanides

$-0.627$

0.114

$30.380 \quad 0.000^{*}$

- Meglitinides

$-0.115$

0.125

$0.840 \quad 0.359$

Constant

1.866

0.146

$164.030 \quad 0.000$

*significance at the 0.05 level

PAAS: Physician Assured Access System

\section{Model fit statistics:}

-2loglikelhiood $=7956.926 ; \chi^{2}=197.66 ; p=0.000$

Dispersion estimate: $0.1987 ; \chi^{2}=943.91 ; \mathrm{p}=0.000$ 


\section{Discussion for Objective 7}

The study results demonstrated that patients with depression had nearly $21.00 \%$ higher type 2 diabetes related costs as compared to patients without depression. These estimates were obtained after adjusting for a number of baseline confounding factors such as demographics, co-morbidity, diabetes severity, and interaction with health care providers. This is to our knowledge the first study in literature to examine the impact of depression on type 2 diabetes related utilization and expenditures. Adjusted smearing estimates indicated that an excess of $\$ 451.27$ type 2 diabetes costs were attributable to presence of depression in the 12-month follow up period.

Most of the studies in this area have examined the impact of depression on total health care costs. One of the reasons that our study examined type 2 diabetes related costs was that we hypothesized that excess type 2 diabetes related costs in depressed patients could be attributable to the impact of depression on decreasing adherence with oral hypoglycemics. Also, our study examined the breakdown of these excess expenditures in detail. This can help us identify cost drivers and provide an explanation for any observed differences in type 2 diabetes expenditures. Our results indicated that increased type 2 diabetes costs in patients with depression were primarily due to increased probability of diabetes related ER/hospitalization episodes. This information may be an important lead for exploring future research in this area. 


\section{$\underline{\text { Results for Objective } 8}$}

To estimate the impact of preexisting depression on overall health care utilization and costs

All medical and prescription claims in the follow up period were identified for this analysis, irrespective of diagnosis codes (except those due to depressive diagnosis). The costs were also examined separately in terms of ER/ hospitalization costs, outpatient costs, and pharmacy costs. Along with expenditures in terms of dollar amounts, overall utilization such as number of ER/hospitalization episodes, number of physician office visits, and number of prescriptions were also compared between depressed and nondepressed patients.

\section{Overall health care costs}

Univariate results for the overall health care costs in the 12-month follow up period are reported in Table 26. The results indicated that health care costs were significantly higher for depressed patients $(\$ 9,809.07 \pm 12,293.26)$ as compared to nondepressed patients $(\$ 6,833.37 \pm 12,369.62)$. Significant differences were observed in all categories of costs such as outpatient costs, ER/hospitalization costs, and prescription costs. Patients with depression ( $\$ 3,057.74 \pm 7,764.26)$ had significantly higher ER/hospitalization costs as compared to non-depressed patients $(\$ 1,909.12 \pm 6,429.12)$. Outpatient costs were also higher for depressed patients $(\$ 3,654.98 \pm 7087.62)$ as compared to non-depressed patients $(\$ 2,808.49 \pm 9,424.36)$. Differences in prescription costs were also observed with depressed patients $(\$ 3,096.35 \pm 2,479.97)$ incurring significantly higher prescription costs as compared to non-depressed patients $(\$ 2,115.76$ $\pm 1,912.47)$. 
Semi-log Ordinary Least Squares (OLS) regression techniques were used for the estimation of the impact of preexisting depression on total costs associated with type 2 diabetes controlling for other confounding factors. The semi-log OLS model was found to be significant with a R-square value of $36.90 \%$. The model results are summarized in Tables 27. Robust standard errors are reported as the model was found to be heteroskedastic. Results of the multivariate model indicated that controlling for confounding covariates, patients with depression incurred $32.11 \%$ higher health care costs as compared to non-depressed patients in the 12-month follow up period $(\mathrm{p}=0.000)$. Retransformation using smearing estimates indicated that an excess of $\$ 1,864.52$ in total health care costs (excluding those due to depressive diagnosis) are due to depression. In models where the dependent variables are specific costs such as those due to ER/hospitalization visits, a two-part model was used in this case to estimate the impact of depression on ER/hospitalization (Tables 28A-28B). An initial multivariate logistic regression model to estimate the impact of depression on a ER/hospitalization episode demonstrated that depressed patients were nearly 1.7 times more likely to have a $\mathrm{ER} /$ hospitalization episode as compared to non-depressed patients $(\mathrm{p}=0.000)$. A subsequent semi-logarithmic OLS model, which was conducted only on those patients having an ER/hospitalization episode revealed no significant differences in ER/hospitalization costs between depressed and non-depressed patients.

As all patients incurred outpatient and prescription costs, semi-log OLS models were used to estimate the impact of depression on these costs in a multivariate framework. The semi-log OLS model for outpatient costs was found to be significant with a R-square value of $28.00 \%$. The model results are summarized in Table 29. 
Results of the multivariate model indicated that controlling for confounding covariates, patients with depression incurred nearly $38.00 \%$ higher outpatient costs as compared to non-depressed patients in the 12-month follow up period $(\mathrm{p}=0.000)$. Retransformation using smearing estimates indicated that an excess of $\$ 732.65$ in total outpatient costs (excluding those due to depressive diagnosis) were attributed to depression.

The semi-log OLS model for prescription costs was found to be significant with a R-square value of $33.5 \%$. The model results are summarized in Table 30. Results of the multivariate model indicated that controlling for confounding covariates, patients with depression incurred nearly $30.85 \%$ higher prescription costs as compared to nondepressed patients in the 12-month follow up period $(\mathrm{p}=0.000)$. Retransformation using smearing estimates indicated that an excess of $\$ 627.75$ in total prescription costs (excluding those due to depressive diagnosis) were attributed to depression.

\section{Health care utilization}

In the 12-month follow up period, significant differences were observed in terms of all the utilization related variables (Table 26$)$. Depressed patients $(2.33 \pm 3.88)$ had twice as many ER/hospitalization episodes as compared to non-depressed patients (1.14 $\pm 2.20)$. Patients with depression $(71.35 \pm 36.42)$ also had significantly higher number of prescriptions as compared to non-depressed patients $(53.79 \pm 32.99)$. Univariate results also indicated that the number of physician office visits were significantly higher for depressed patients $(10.44 \pm 9.98)$ as compared to non-depressed patients $(6.63 \pm 6.27)$.

Negative binomial models were used for estimation of all the utilization related variables. Results of the negative binomial models indicated that significant differences were observed on all the utilization parameters between the two groups in a multivariate 
framework (Tables 31-33). Multivariate results indicated that controlling baseline covariates, patients with depression had $48.14 \%$ higher number of ER/Hospitalization episodes as compared to non-depressed patients $(p=0.000)$. Patients with depression also had $28.78 \%$ higher number of physician office visits as compared to non-depressed patients $(\mathrm{p}=0.000)$. Similar results were observed in terms of prescription utilization with depression patients filling $16.76 \%$ higher number of prescriptions in the 12 -month follow up period $(\mathrm{p}=0.000)$. 
Table 26: Utilization and expenditures for overall health care (excluding those due to depression ) in the 12-month follow up period

$\begin{array}{cccl}\text { Depressed } & \text { Non-depressed } & \text { Mann-Whitney } & \text { Sig. } \\ (\text { Mean }+ \text { S.D. }) & (\text { Mean } \pm S . D .) & \text { Utest } & (p) \\ (N=471) & (N=855) & & \\ & & & \end{array}$

Overall health care costs (in dollars)

Outpatient costs

$$
\begin{array}{r}
3,654.98 \\
+7,087.62
\end{array}
$$

ER/Hosp costs

$$
3,057.74
$$$$
\pm 7,764.26
$$

Pharmacy costs

$$
3,096.35
$$$$
\pm 2,479.97
$$

Total costs

$$
\begin{array}{r}
9,809.07 \\
+12,293.26
\end{array}
$$

Overall health care utilization

Number of physician $\quad 10.44 \pm 9.98$ office visits

Number of ER/hosp $\quad 2.33 \pm 3.88$ visits

Number of Rxs

$$
71.35 \pm 36.42
$$

$$
2,808.49
$$$$
+9,424.36
$$

$$
1,909.12
$$$$
\pm 6,429.12
$$

$$
\begin{array}{r}
2,115.76 \\
+1,912.47
\end{array}
$$

$6,833.37$

$+12,369.62$
$146647.00 \quad 0.000^{*}$

$158711.50 \quad 0.000 *$

$141544.00 \quad 0.000 *$

$140387.00 \quad 0.000 *$

$6.63 \pm 6.27$

$149043.50 \quad 0.000 *$

$1.14 \pm 2.20$

$154814.50 \quad 0.000 *$

*Significance at the 0.05 level 
Table 27: Semi-log Ordinary Least Squares (OLS) regression model for the impact of depression on total healthcare costs (excluding depression costs)

Beta Robust Test statistic Sig.

S.E.

( $t)$ (p)

\section{Depression}

0.280

0.055

$5.1330 .000^{*}$

Demographic characteristics

Age (in years)

$$
0.006 \quad 0.003
$$

$2.2450 .025^{*}$

Males (ref: females)

$-0.025$

0.055

$-0.462 \quad 0.644$

Whites (ref: non-whites)

$$
0.056
$$

0.099

$0.566 \quad 0.572$

Urban (ref: rural)

\begin{tabular}{ll}
$-0.203-0.093$ \\
\hline
\end{tabular}

$-2.1850 .029 *$

Year of index prescription (ref: 2001)

- 1998

- 1999

0.164

0.174

0.121

1.350

1.520

0.156

0.115

1.523

0.177

- 2000

0.103

0.129

0.128

Co-morbid conditions in the pre period

Number of ER/hosp visits

$0.023 \quad 0.012 \quad 1.9350 .053^{*}$

Total health costs

$0.000 \quad 0.000$

$9.9670 .000^{*}$

Charlson co-morbidity

0.060

0.027

$2.2040 .028^{*}$

Number of therapeutic classes

$0.107 \quad 0.015$

$7.1620 .000^{*}$

of medications

Cardio-vascular

0.029

0.055

$0.524 \quad 0.601$

Cancer

$-0.134$

0.114

$-1.172 \quad 0.241$

Asthma

0.108

0.066

$1.654 \quad 0.098$

Ulcers

0.209

0.138

$1.515 \quad 0.130$

Provider interaction in the pre period

PAAS enrollment
0.062
0.078
$0.801 \quad 0.423$

Number of pharmacies visited (ref: multiple pharmacies)
- No pharmacy
$-0.156$
0.163
$-0.960 \quad 0.337$
- Single pharmacy
$-0.043$
0.050
$-0.863 \quad 0.388$ 
Table 27: Semi-log Ordinary Least Squares (OLS) regression model for the impact of depression on total healthcare costs (excluding depression costs) (Contd.)

$\begin{array}{cccc}\text { Beta } & \text { Robust } & \text { Test statistic } & \text { Sig. } \\ & \text { S.E. } & (t) & (p)\end{array}$

Diabetes severity

Index oral hypoglycemic prescription by: (ref: Family medicine)

$\begin{array}{lllll}\text { - Endocrinologist } & 0.188 & 0.187 & 1.008 & 0.314 \\ \text { - Internal medicine } & 0.053 & 0.053 & 0.989 & 0.323\end{array}$

$\begin{array}{lllll}\text { Total diabetes costs } & 0.000 & 0.000 & -1.970 & 0.049^{*}\end{array}$

$\begin{array}{lllll}\text { Number of diabetes related } & 0.141 & 0.066 & 2.133 & 0.033^{*}\end{array}$

ER/Hosp visits

$\begin{array}{lllll}\text { Gap between index diagnosis } & 0.000 & 0.000 & 1.033 & 0.302\end{array}$ and prescription

$\begin{array}{lllll}\text { Initial polytherapy } & 0.228 & 0.086 & 2.649 & 0.008^{*}\end{array}$

Index class of oral hypoglycemic (ref: Sulfonyl urea)

$\begin{array}{lllll}\text { - Thiazolidenedione } & 0.182 & 0.103 & 1.765 & 0.078\end{array}$

$\begin{array}{lllll}\text { - Biguanides } & 0.058 & 0.052 & 1.129 & 0.259\end{array}$

$\begin{array}{lllll}\text { - Alpha-glucosidase } & 0.060 & 0.143 & 0.423 & 0.672\end{array}$

$\begin{array}{lllll}\text { - Sulfonyl-biguanides } & -0.296 & 0.164 & -1.804 & 0.071\end{array}$

$\begin{array}{lllll}- \text { Meglitinides } & 0.063 & 0.176 & 0.357 & 0.721\end{array}$

\begin{tabular}{lllll} 
Constant & 7.357 & 0.231 & 31.907 & 0.000 \\
\hline
\end{tabular}

* Significance at the 0.05 level

PAAS: Physician Assured Access System

\section{Model fit statistics:}

R-square: $36.9 \% ; \mathrm{F}=24.536 ; \mathrm{P}=0.000$

Durbin-Watson: 1.931

White test for heteroskedasticity: Chi-square $=495.31 ; \mathrm{P}=0.0001$ 
TWO-PART MODEL-PART 1

Table 28A: Logistic regression for the impact of depression on having an ER/Hospitalization episode (excluding depression)

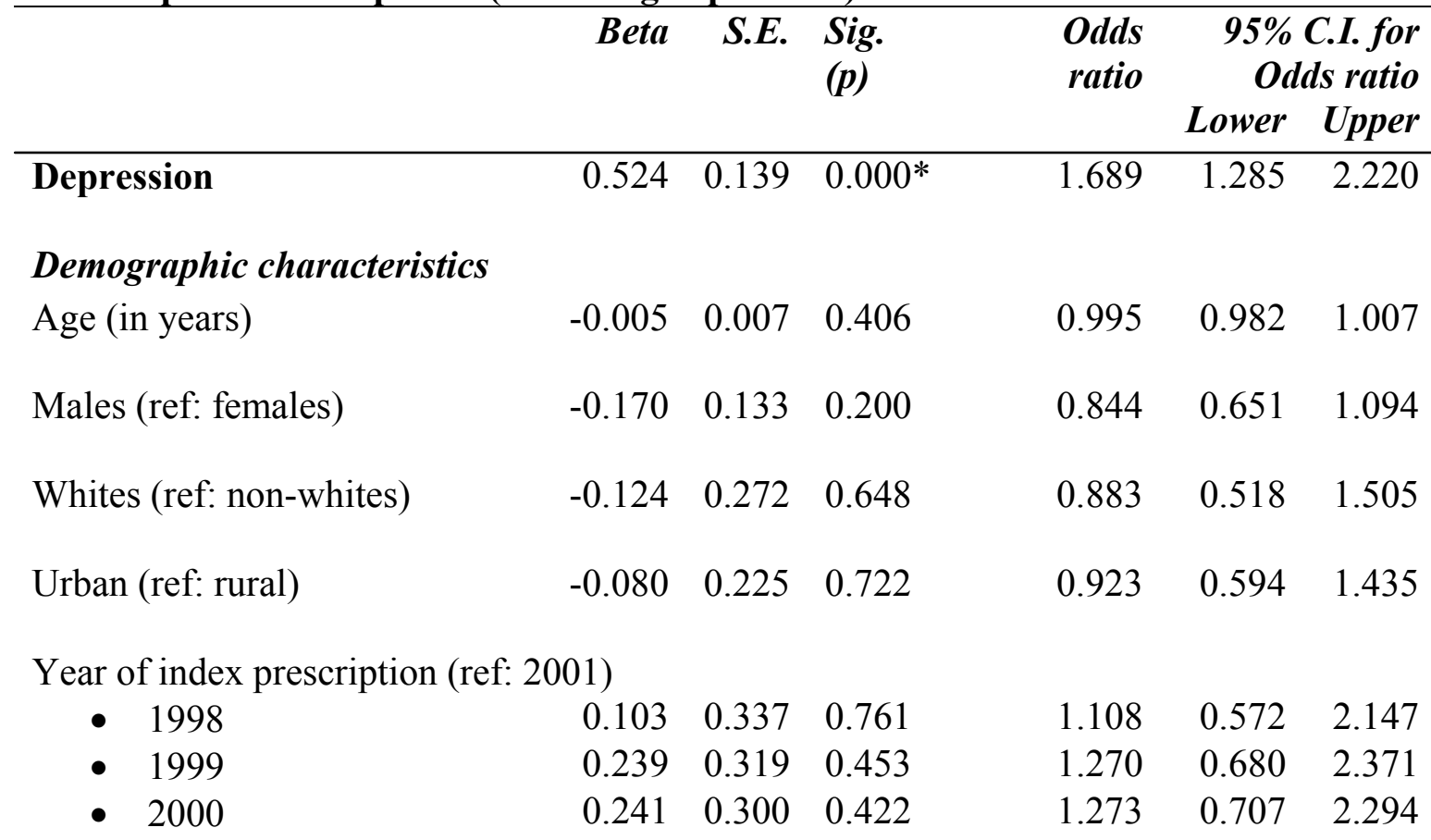

Co-morbid conditions in the pre period

Number of ER/hosp visits$$
0.411
$$

$0.0530 .000^{*}$

$1.508 \quad 1.359 \quad 1.674$

Total health costs

$\begin{array}{lll}0.000 & 0.000 & 0.511\end{array}$

$\begin{array}{lll}1.000 & 1.000 \quad 1.000\end{array}$

Charlson co-morbidity

$0.198 \quad 0.085 \quad 0.019 *$

$\begin{array}{lll}1.219 & 1.033 & 1.440\end{array}$

Number of therapeutic classes of medications

Cardio-vascular

$\begin{array}{lll}0.041 & 0.040 & 0.310\end{array}$

1.04

0.963

1.126

$\begin{array}{lll}-0.238 & 0.140 & 0.090\end{array}$

0.788

$0.599 \quad 1.038$

Cancer

$\begin{array}{lll}-0.449 & 0.364 & 0.217\end{array}$

0.638

$0.313 \quad 1.303$

Asthma

$\begin{array}{lll}0.355 & 0.198 & 0.073\end{array}$

1.427

$0.968 \quad 2.103$

Ulcers

$0.291 \quad 0.435 \quad 0.504$

1.338

$0.570 \quad 3.140$

Provider interaction in the pre period

\begin{tabular}{|c|c|c|c|c|c|c|}
\hline PAAS enrollment & 0.157 & 0.195 & 0.422 & 1.169 & 0.798 & 1.713 \\
\hline \multicolumn{7}{|c|}{ Number of pharmacies visited (ref: multiple pharmacies) } \\
\hline - No $\mathrm{p}$ & 0.119 & 0.308 & 0.699 & 1.126 & 0.616 & 2.058 \\
\hline - Single pharmacy & -0.131 & 0.129 & 0.309 & 0.877 & 0.681 & 1.129 \\
\hline
\end{tabular}


Table 28A: Logistic regression for the impact of depression on having an ER/hospitalization episode (excluding depression) (Contd.)

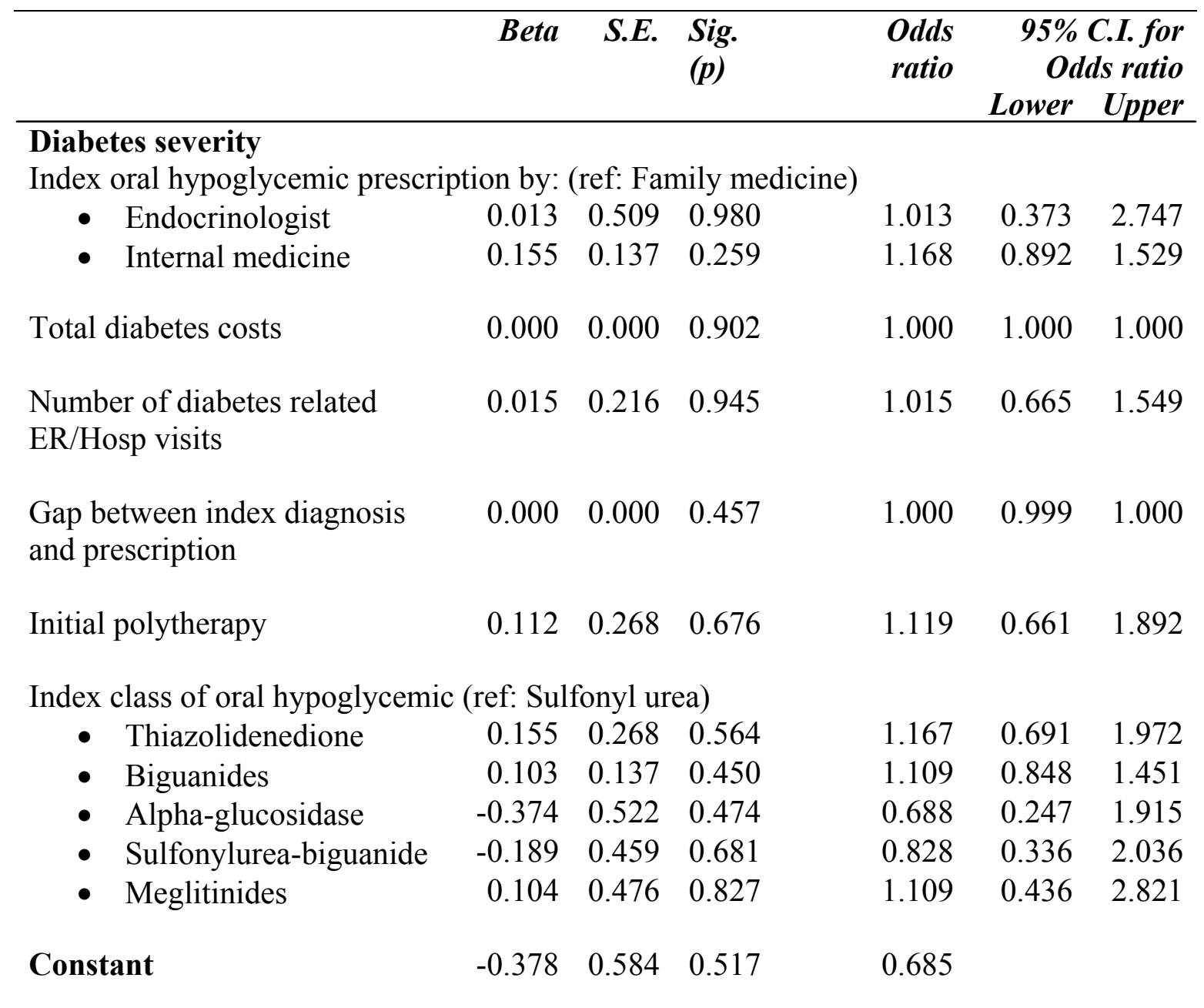

*Significance at the 0.05 level

PAAS: Physician Assured Access System

Model fit statistics:

Pseudo R-square $=21.8 \%$

-2 Log Likelihood $=1559.939 ; \chi^{2}=230.150, p=0.000$

Hosmer and Lemeshow's goodness of fit: $\chi^{2}=16.207$; $p=0.040$ 
TWO-PART MODEL-PART 2

Table 28B: Semi-log Ordinary Least Squares (OLS) regression model for the impact of depression on total ER/hospitalization costs (excluding depression)

\section{Beta S.E. Test statistic Sig.}

Depression

0.154

0.155

$0.994 \quad 0.321$

Demographic characteristics

Age (in years)

$0.022 \quad 0.007$

$2.890 \quad 0.004^{*}$

Males (ref: females)

$0.194 \quad 0.159$

$1.217 \quad 0.224$

Whites (ref: non-whites)

$0.238 \quad 0.316$

$0.752 \quad 0.452$

Urban (ref: rural)

$\begin{array}{llll}-0.170 & 0.265 & -0.643 & 0.520\end{array}$

Year of index prescription (ref: 2001)

- 1998

0.605

0.379

$\begin{array}{ll}1.599 & 0.110\end{array}$

- 1999

0.462

0.356

$1.300 \quad 0.194$

- 2000

0.267

0.339

$\begin{array}{ll}0.786 & 0.432\end{array}$

Co-morbid conditions in the pre period

Number of ER/hosp visits

Total health costs

$0.087 \quad 0.023$

3.77

$0.000^{*}$

Total health costs

$0.000 \quad 0.000$

$2.1190 .034^{*}$

Charlson co-morbidity

$0.079 \quad 0.075$

$1.056 \quad 0.291$

Number of therapeutic classes

$0.115 \quad 0.044$

$2.6220 .009 *$

of medications

Cardio-vascular

0.157

0.162

$0.966 \quad 0.334$

Cancer

$-0.442$

0.383

$-1.155 \quad 0.248$

Asthma

$-0.030$

0.190

$-0.155 \quad 0.877$

Ulcers

0.873

0.365

$2.3880 .017^{*}$

Provider interaction in the pre period

PAAS enrollment

$\begin{array}{llll}-0.009 & 0.214 & -0.043 & 0.966\end{array}$

Number of pharmacies visited (ref: multiple pharmacies)

- No pharmacy

- Single pharmacy
0.543

$-0.045$
0.401

0.150 $\begin{array}{ll}1.355 & 0.176\end{array}$

$-0.300 \quad 0.764$ 
Table 28B: Semi-log Ordinary Least Squares (OLS) regression model for the impact of depression on total ER/hospitalization costs (excluding depression)

Beta S.E. Test statistic Sig.

( $t$ ( $\quad(p)$

Diabetes severity

Index oral hypoglycemic prescription by: (ref: Family medicine)

$\begin{array}{lllll}\text { - Endocrinologist } & 0.516 & 0.536 & 0.963 & 0.336\end{array}$

- Internal medicine $\quad-0.007 \quad 0.151 \quad-0.045 \quad 0.964$

Total diabetes costs

$\begin{array}{llll}0.000 & 0.000 \quad-0.091 & 0.928\end{array}$

Number of diabetes related

$\begin{array}{llll}0.288 & 0.189 & 1.526 & 0.128\end{array}$

ER/Hosp visits

$\begin{array}{lllll}\text { Gap between index diagnosis } & 0.000 & 0.000 & 0.503 & 0.615\end{array}$ and prescription

$\begin{array}{lllll}\text { Initial polytherapy } & -0.230 & 0.323 & -0.711 & 0.478\end{array}$

Index class of oral hypoglycemic (ref: Sulfonyl urea)

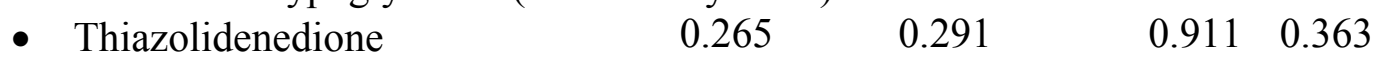

$\begin{array}{lllll}\text { - Biguanides } & 0.019 & 0.158 & 0.123 & 0.902\end{array}$

$\begin{array}{lllll}\text { - Alpha-glucosidase } & -0.935 & 0.587 & -1.593 & 0.112\end{array}$

$\begin{array}{lllll}\text { - Sulfonyl-biguanides } & 0.062 & 0.559 & 0.111 & 0.912\end{array}$

$\begin{array}{lllll}\text { - Meglitinides } & -0.461 & 0.563 & -0.819 & 0.413\end{array}$

$\begin{array}{lllll}\text { Constant } & 4.436 & 0.660 & 6.722 & 0.000\end{array}$

*Significance at the 0.05 level

PAAS: Physician Assured Access System

Model fit statistics:

R-square: $17.0 \% ; \mathrm{F}=4.321 ; \mathrm{P}=0.000$

Durbin-Watson: 2.109

White test for heteroskedasticity: $\chi^{2}=408.66 ; p=0.3850$ 
Table 29: Semi-log Ordinary Least Squares (OLS) regression model for the impact of depression on total outpatient costs (excluding depression)

\begin{tabular}{|c|c|c|}
\hline eta & S.E. & $\begin{array}{c}\text { Test statistic } \\
(t)\end{array}$ \\
\hline
\end{tabular}

Depression

$0.325 \quad 0.077$

$4.2260 .000 *$

Demographic characteristics

Age (in years)

$\begin{array}{llll}-0.006 & 0.004 & -1.607 & 0.108\end{array}$

Males (ref: females)

$\begin{array}{llll}-0.168 & 0.074 & -2.278 & 0.023^{*}\end{array}$

Whites (ref: non-whites)

0.070

0.151

$0.464 \quad 0.643$

Urban (ref: rural)

$\begin{array}{llll}-0.271 & 0.126 & -2.158 & 0.031^{*}\end{array}$

Year of index prescription (ref: 2001)
- 1998
0.315
0.183
$\begin{array}{ll}1.720 & 0.086\end{array}$
- 1999
0.203
0.172
$1.177 \quad 0.240$
- 2000
0.294
0.162
$\begin{array}{ll}1.808 & 0.071\end{array}$

Co-morbid conditions in the pre period

Number of ER/hosp visits

$\begin{array}{llll}0.013 & 0.014 & 0.951 & 0.342\end{array}$

Total health costs

$0.000 \quad 0.000$

$14.360 \quad 0.000^{*}$

Charlson co-morbidity

$0.092 \quad 0.041$

$2.2560 .024^{*}$

Number of therapeutic classes

$0.008 \quad 0.022$

$0.345 \quad 0.730$

of medications

Cardio-vascular

$0.187 \quad 0.078$

$2.4010 .017^{*}$

Cancer

0.073

0.192

$0.379 \quad 0.705$

Asthma

$0.233 \quad 0.104$

$2.2430 .025^{*}$

Ulcers

$\begin{array}{ll}0.363 & 0.209\end{array}$

$\begin{array}{ll}1.736 & 0.083\end{array}$

Provider interaction in the pre period

PAAS enrollment
0.312
0.106
$2.9370 .003 *$

Number of pharmacies visited (ref: multiple pharmacies)
- No pharmacy
$-0.263$
0.176
$-1.494 \quad 0.135$
- Single pharmacy
$-0.051 \quad 0.072$
$-0.712 \quad 0.476$ 
Table 29: Semi-log Ordinary Least Squares (OLS) regression model for the impact of depression on total outpatient costs (excluding depression) (Contd.)

Beta S.E. Test statistic Sig.

( $t)$

(p)

Diabetes severity

Index oral hypoglycemic prescription by: (ref: Family medicine)
- Endocrinologist
$0.183 \quad 0.276$
$0.665 \quad 0.506$
- Internal medicine
0.033
0.075
$0.435 \quad 0.663$

Total diabetes costs

$0.000 \quad 1.6 \mathrm{E}-05$

$-1.291 \quad 0.197$

Number of diabetes related

$0.046 \quad 0.10086$

$0.456 \quad 0.648$

ER/Hosp visits

Gap between index diagnosis

$0.000 \quad 0.0002$

$1.455 \quad 0.146$

and prescription

Initial polytherapy

$0.277 \quad 0.15132$

$1.834 \quad 0.067$

Index class of oral hypoglycemic (ref: Sulfonyl urea)

- Thiazolidenedione

- Biguanides

- Alpha-glucosidase

- Sulfonyl-biguanides

- Meglitinides
$-0.099$

$-0.046$

0.244

$-0.430$

$-0.094$

0.14598

$\begin{array}{ll}-0.676 & 0.499\end{array}$

0.07555

$\begin{array}{ll}-0.614 & 0.539\end{array}$

0.27655

$\begin{array}{ll}0.881 & 0.379\end{array}$

$\begin{array}{ll}-1.665 & 0.096\end{array}$

$-0.346 \quad 0.729$

Constant

6.640

0.25836

0.27056

*significance at the 0.05 level

PAAS: Physician Assured Access System

\section{Model fit statistics:}

R-square: $28.0 \% ; \mathrm{F}=16.254 ; \mathrm{P}=0.000$

Durbin-Watson: 1.925

White test for heteroskedasticity: $\chi^{2}=390.70 ; p=0.9330$ 
Table 30: Semi-log Ordinary Least Squares (OLS) regression model for the impact of depression on total prescription costs (excluding depression)

$\begin{array}{llll}\text { Beta } & \text { S.E. Test statistic Sig. }\end{array}$

(t)

(p)

Depression

0.270

0.048

$5.6680 .000^{*}$

Demographic characteristics

Age (in years)

$0.009 \quad 0.002$

$3.9690 .000 *$

Males (ref: females)

$-0.006 \quad 0.046$

$-0.1320 .895$

Whites (ref: non-whites)

$0.030 \quad 0.094$

$0.319 \quad 0.750$

Urban (ref: rural)

$\begin{array}{llll}-0.057 & 0.078 & -0.735 & 0.463\end{array}$

Year of index prescription (ref: 2001)

- 1998

0.018

0.113

$\begin{array}{ll}0.159 & 0.874\end{array}$

- 1999

0.121

0.107

$\begin{array}{ll}1.134 & 0.257\end{array}$

- 2000

0.068

0.101

$0.673 \quad 0.501$

Co-morbid conditions in the pre period

Number of ER/hosp visits

Total health costs

0.000

0.000

$0.008 \quad 0.025$

0.009

$-0.700$

0.484

Charlson co-morbidity

0.183

0.013

$5.7150 .000^{*}$

Number of therapeutic classes

of medications

Cardio-vascular

0.068

0.048

$1.406 \quad 0.160$

Cancer

$-0.129$

0.119

$-1.081 \quad 0.280$

Asthma

0.059

0.064

$0.910 \quad 0.363$

Ulcers

0.010

0.128

$\begin{array}{ll}0.079 & 0.937\end{array}$

Provider interaction in the pre period

PAAS enrollment

$\begin{array}{llll}-0.050 & 0.066 & -0.757 & 0.449\end{array}$

Number of pharmacies visited (ref: multiple pharmacies)
- No pharmacy
$-0.275$
0.109
$-2.5280 .012 *$
- Single pharmacy
$-0.048$
0.044
$\begin{array}{ll}-1.087 & 0.277\end{array}$ 
Table 30: Semi-log Ordinary Least Squares (OLS) regression model for the impact of depression on total prescription costs (excluding depression) (Contd.)

Beta S.E. $\quad$ Test statistic Sig.

(t) (p)

Diabetes severity

Index oral hypoglycemic prescription by: (ref: Family medicine)
- Endocrinologist
0.133
0.171
$0.776 \quad 0.438$

- Internal medicine

0.014

0.046

$0.298 \quad 0.766$

Total diabetes costs

$0.000 \quad 0.000$

$0.028 \quad 0.978$

Number of diabetes related

$0.025 \quad 0.063$

$0.396 \quad 0.692$

ER/Hosp visits

\begin{tabular}{lccrl}
$\begin{array}{l}\text { Gap between index diagnosis } \\
\text { and prescription }\end{array}$ & 0.000 & 0.000 & 0.979 & 0.328 \\
& & & & \\
Initial polytherapy & 0.386 & 0.094 & 4.110 & $0.000^{*}$ \\
& & & & \\
$\begin{array}{l}\text { Index class of oral hypoglycemic (ref: Sulfonyl urea) } \\
\text { • Thiazolidenedione }\end{array}$ & 0.336 & 0.090 & 3.728 & $0.000^{*}$ \\
- Biguanides & 0.126 & 0.047 & 2.680 & $0.007^{*}$ \\
- Alpha-glucosidase & 0.117 & 0.172 & 0.682 & 0.495 \\
- Sulfonyl-biguanides & -0.223 & 0.160 & -1.391 & 0.164 \\
- Meglitinides & 0.224 & 0.168 & 1.334 & 0.182 \\
Constant & & & & \\
\hline
\end{tabular}

*significance at the 0.05 level

PAAS: Physician Assured Access System

\section{Model fit statistics:}

R-square: $33.5 \% ; \mathrm{F}=21.204 ; \mathrm{P}=0.000$

Durbin-Watson: 2.004

White test for heteroskedasticity: $\chi^{2}=459.76 ; p=0.1633$ 
Table 31: Negative binomial model for the impact of depression on number of overall ER/hospitalization episodes (excluding depression)

\section{Estimate S.E. Test statistic Sig. (Chi-square) (p)}

Depression

$0.393 \quad 0.085$

$21.200 \quad 0.000^{*}$

Demographic characteristics

Age (in years)

$\begin{array}{ll}-0.002 & 0.004\end{array}$

$0.290 \quad 0.592$

Males (ref: females)

$-0.002$

0.085

$0.000 \quad 0.981$

Whites (ref: non-whites)

0.044

0.181

$0.060 \quad 0.810$

Urban (ref: rural)

$-0.009$

0.146

$0.000 \quad 0.948$

Year of index prescription (ref: 2001)
- 1998
0.126
0.216
$\begin{array}{ll}0.340 & 0.560\end{array}$
- 1999
0.059
0.204
$\begin{array}{ll}0.080 & 0.773\end{array}$
- 2000
0.132
0.195
$\begin{array}{ll}0.460 & 0.499\end{array}$

Co-morbid conditions in the pre period

Number of ER/hosp visits

$\begin{array}{llll}0.197 & 0.017 & 127.470 & 0.000 *\end{array}$

Total health costs

$0.000 \quad 0.000$

$0.160 \quad 0.688$

Charlson co-morbidity

$0.089 \quad 0.043$

$4.390 \quad 0.036 *$

Number of therapeutic classes

0.052

0.024

$4.630 \quad 0.031 *$

of medications

Cardio-vascular

$\begin{array}{ll}-0.185 & 0.089\end{array}$

$4.350 \quad 0.037^{*}$

Cancer

$-0.210$

0.216

$0.950 \quad 0.330$

Asthma

0.073

0.110

$0.440 \quad 0.508$

Ulcers

0.099

0.219

$0.210 \quad 0.650$

Provider interaction in the pre period

PAAS enrollment
$-0.060$
0.123
$0.240 \quad 0.626$

Number of pharmacies visited (ref: multiple pharmacies)
- No pharmacy
0.201
0.204
$0.970 \quad 0.324$
- Single pharmacy
$-0.173 \quad 0.083$
$4.350 \quad 0.037$ * 
Table 31: Negative binomial model for the impact of depression on number of overall ER/hospitalization episodes (excluding depression) (Contd.)

\begin{tabular}{|c|c|c|c|c|}
\hline & Estimate & S.E. & $\begin{array}{l}\text { Test statistic } \\
\text { (Chi-square) }\end{array}$ & $\begin{array}{l}\text { Sig. } \\
\text { (p) }\end{array}$ \\
\hline \multicolumn{5}{|l|}{ Diabetes severity } \\
\hline \multicolumn{5}{|c|}{ Index oral hypoglycemic prescription by: (ref: Family medicine) } \\
\hline - Endocrinologist & 0.118 & 0.298 & 0.160 & 0.692 \\
\hline - Internal medicine & 0.103 & 0.085 & 1.450 & 0.229 \\
\hline Total diabetes costs & 0.000 & 0.000 & 2.130 & 0.144 \\
\hline $\begin{array}{l}\text { Number of diabetes related } \\
\text { ER/Hosp visits }\end{array}$ & 0.206 & 0.102 & 4.100 & $0.043^{*}$ \\
\hline $\begin{array}{l}\text { Gap between index diagnosis } \\
\text { and prescription }\end{array}$ & 0.000 & 0.000 & 0.270 & 0.604 \\
\hline Initial polytherapy & 0.230 & 0.170 & 1.840 & 0.175 \\
\hline \multicolumn{5}{|c|}{ Index class of oral hypoglycemic (ref: Sulfonyl urea) } \\
\hline - Thiazolidenedione & 0.172 & 0.161 & 1.140 & 0.286 \\
\hline - Biguanides & -0.003 & 0.086 & 0.000 & 0.971 \\
\hline - Alpha-glucosidase & -0.641 & 0.355 & 3.260 & 0.071 \\
\hline - Sulfonyl-biguanides & -0.500 & 0.309 & 2.620 & 0.106 \\
\hline - Meglitinides & -0.134 & 0.323 & 0.170 & 0.678 \\
\hline Constant & -0.306 & 0.369 & 0.690 & 0.407 \\
\hline
\end{tabular}

*significance at the 0.05 level

PAAS: Physician Assured Access System

\section{Model fit statistics:}

-2loglikelihood $=3948.0036 \chi^{2}=377.59 ; p=0.00$

Dispersion estimate: $1.0099 ; \chi^{2}=799.89 ; p=0.000$ 
Table 32: Negative binomial model for the impact of depression on number of overall physician office visits (excluding depression)

$\begin{array}{llrl}\text { Estimate } & \text { S.E. } & \begin{array}{r}\text { Test statistic } \\ \text { (Chi-square) }\end{array} & \text { Sig. } \\ \end{array}$

$\begin{array}{lllll}\text { Depression } & 0.253 & 0.060 & 17.770 & 0.000 *\end{array}$

Demographic characteristics

Age (in years)

$\begin{array}{llll}0.002 & 0.003 & 0.260 & 0.613\end{array}$

Males (ref: females)

$\begin{array}{llll}-0.121 & 0.058 & 4.330 & 0.037 *\end{array}$

Whites (ref: non-whites)

0.107

0.121

$0.770 \quad 0.380$

Urban (ref: rural)

$-0.083$

0.100

$0.690 \quad 0.406$

Year of index prescription (ref: 2001)

- 1998

$-0.077$

0.145

$0.280 \quad 0.596$

- 1999

0.021

0.136

$0.020 \quad 0.879$

- 2000

0.059

0.129

$0.210 \quad 0.649$

Co-morbid conditions in the pre period

Number of ER/hosp visits

$\begin{array}{llll}0.038 & 0.012 & 10.700 & 0.001 *\end{array}$

Total health costs

$0.000 \quad 0.000$

$3.250 \quad 0.072$

Charlson co-morbidity

$0.046 \quad 0.030$

$2.350 \quad 0.125$

Number of therapeutic classes

0.043

0.017

$6.820 \quad 0.009 *$

of medications

Cardio-vascular

$0.069 \quad 0.061$

$\begin{array}{ll}1.310 & 0.253\end{array}$

Cancer

0.042

0.150

$0.080 \quad 0.781$

Asthma

$0.053 \quad 0.078$

$0.470 \quad 0.495$

Ulcers

0.402

0.156

$6.630 \quad 0.010^{*}$

Provider interaction in the pre period

PAAS enrollment
0.076
0.083
$0.830 \quad 0.362$

Number of pharmacies visited (ref: multiple pharmacies)
- No pharmacy
$-0.316$
0.146
$4.720 \quad 0.030 *$
- Single pharmacy
$-0.089$
0.057
$2.420 \quad 0.120$ 
Table 32: Negative binomial model for the impact of depression on number of overall physician office visits (excluding depression) (Contd.)

\begin{tabular}{|c|c|c|c|c|}
\hline & Estimate & S.E. & $\begin{array}{l}\text { Test statistic } \\
\text { (Chi-square) }\end{array}$ & $\begin{array}{l}\text { Sig. } \\
(p)\end{array}$ \\
\hline \multicolumn{5}{|l|}{ Diabetes severity } \\
\hline \multicolumn{5}{|c|}{ Index oral hypoglycemic prescription by: (ref: Family medicine) } \\
\hline - Endocrinologist & 0.501 & 0.210 & 5.710 & $0.017^{*}$ \\
\hline - Internal medicine & 0.138 & 0.059 & 5.440 & $0.020 *$ \\
\hline Total diabetes costs & 0.000 & 0.000 & 0.000 & 0.951 \\
\hline \multicolumn{4}{|l|}{ ER/Hosp visits } & 0.925 \\
\hline $\begin{array}{l}\text { Gap between index diagnosis } \\
\text { and prescription }\end{array}$ & 0.000 & 0.000 & 0.690 & 0.405 \\
\hline Initial polytherapy & 0.115 & 0.120 & 0.920 & 0.336 \\
\hline \multicolumn{5}{|c|}{ Index class of oral hypoglycemic (ref: Sulfonyl urea) } \\
\hline - Thiazolidenedione & 0.204 & 0.114 & 3.190 & 0.074 \\
\hline - Biguanides & 0.076 & 0.060 & 1.600 & 0.206 \\
\hline - Alpha-glucosidase & 0.396 & 0.213 & 3.460 & 0.063 \\
\hline - Sulfonyl-biguanides & -0.014 & 0.203 & 0.000 & 0.946 \\
\hline - Meglitinides & 0.096 & 0.214 & 0.200 & 0.653 \\
\hline Constant & 1.522 & 0.252 & 36.590 & 0.000 \\
\hline
\end{tabular}

*significance at the 0.05 level

PAAS: Physician Assured Access System

\section{Model fit statistics:}

-2loglikelihood $=7909.38 ; \chi^{2}=205.05 ; p=0.000$

Dispersion estimate: $0.7588 ; \chi^{2}=3819.72 ; p=0.000$ 
Table 33: Negative binomial model for the impact of depression on number of overall prescriptions (excluding depression)

\section{Estimate S.E. Test statistic Sig. (Chi-square) (p)}

Depression

$0.155 \quad 0.031$

$25.390 \quad 0.000^{*}$

Demographic characteristics

Age (in years)

$0.008 \quad 0.002$

$28.870 \quad 0.000 *$

Males (ref: females)

$\begin{array}{ll}-0.079 & 0.029\end{array}$

$7.240 \quad 0.007^{*}$

Whites (ref: non-whites)

0.028

0.061

$0.210 \quad 0.643$

Urban (ref: rural)

$-0.022 \quad 0.050$

$0.180 \quad 0.669$

Year of index prescription (ref: 2001)

- 1998

$\begin{array}{ll}-0.027 & 0.074\end{array}$

$0.140 \quad 0.710$

- 1999

$0.003 \quad 0.069$

$0.000 \quad 0.968$

- 2000

$-0.042$

0.065

$0.420 \quad 0.518$

Co-morbid conditions in the pre period

Number of ER/hosp visits

$\begin{array}{llll}0.001 & 0.006 & 0.030 & 0.862\end{array}$

Total health costs

$0.000 \quad 0.000$

$18.150 \quad 0.000^{*}$

Charlson co-morbidity

$-0.002 \quad 0.016$

$0.010 \quad 0.916$

Number of therapeutic classes

$0.153 \quad 0.009$

$303.320 \quad 0.000 *$

of medications

Cardio-vascular

$0.048 \quad 0.031$

$2.410 \quad 0.120$

Cancer

$-0.146$

0.077

$3.620 \quad 0.057$

Asthma

$0.051 \quad 0.041$

$1.540 \quad 0.215$

Ulcers

0.060

0.082

$0.540 \quad 0.464$

Provider interaction in the pre period

PAAS enrollment
0.012
0.043
$0.070 \quad 0.786$

Number of pharmacies visited (ref: multiple pharmacies)
- No pharmacy
$-0.181$
0.072
$6.260 \quad 0.012 *$
- Single pharmacy
$\begin{array}{ll}-0.059 & 0.029\end{array}$
$4.170 \quad 0.041^{*}$ 
Table 33: Negative binomial model for the impact of depression on number of overall prescriptions (excluding depression) (Contd.)

$\begin{array}{llll}\text { Estimate } & \text { S.E. } & \text { Test statistic } & \text { Sig. }\end{array}$

Diabetes severity

Index oral hypoglycemic prescription by: (ref: Family medicine)
- Endocrinologist
0.142
0.110
$\begin{array}{ll}1.670 & 0.197\end{array}$
- Internal medicine
0.034
0.030
$1.320 \quad 0.251$

Total diabetes costs

$0.000 \quad 0.000$

$0.080 \quad 0.775$

Number of diabetes related

$0.019 \quad 0.041$

$0.220 \quad 0.637$

ER/Hosp visits

Gap between index diagnosis

$0.000 \quad 0.000$

$0.210 \quad 0.648$

and prescription

Initial polytherapy

$0.246 \quad 0.061$

$16.380 \quad 0.000^{*}$

Index class of oral hypoglycemic (ref: Sulfonyl urea)

- Thiazolidenedione $-0.024$

0.058

$\begin{array}{ll}0.170 & 0.683\end{array}$

- Biguanides

$-0.025$

0.030

$0.690 \quad 0.406$

- Alpha-glucosidase

0.027

0.111

$0.060 \quad 0.805$

- Sulfonyl-biguanides

$-0.272$

0.104

$6.880 \quad 0.009^{*}$

- Meglitinides

$-0.132$

0.108

$1.480 \quad 0.224$

Constant

3.205

0.128

$632.110 \quad 0.000$

*Significance at the 0.05 level

PAAS: Physician Assured Access System

\section{Model fit statistics:}

-2loglikelhood: $1192.86 ; \chi^{2}=664.45 ; p=0.000$

Dispersion estimate: $0.2128(0.1956-02316) ; \chi^{2}=3599.54 ; p=0.000$ 


\section{Discussion for Objective 8}

Our study results indicated that patients with depression had nearly $32.10 \%$ higher health care costs as compared to patients without depression. These estimates were obtained after adjusting for a number of baseline confounding factors such as demographics, co-morbidity, diabetes severity, and interaction with health care providers. A few studies in the literature have examined the impact of depression on health care costs in patients with type 2 diabetes. Ciechanowski and associates ${ }^{28}$ showed that after controlling for demographics, medical comorbidity, and diabetes severity, higher levels of depression severity were associated with a significantly greater probability of having an emergency department, primary care, specialty care, medical inpatient, and mental health costs compared to patients with low severity depression. The total costs of the high tertile group were $\$ 3,654$ versus $\$ 2,653$ in the medium depression tertile and $\$ 2,094$ in the low tertile groups. However, these reported costs were unadjusted and were calculated for a six-month period. Also, it was a population-based study from two primary care clinics with relatively small sample sizes, especially for assessing costs. Egede and associates ${ }^{125}$ used the Medical Expenditure Panel Survey (MEPS) and showed that patients with depression and diabetes incurred 4.5 times greater health care costs than patients with diabetes alone. However, the numbers reported for excess costs seemed unreasonably large thus raising concerns regarding the validity of the study results. Both the above studies relied on self-reported data and had small sample sizes. Most importantly, these studies examined total health care costs that included costs due to depressive diagnosis, which will be higher for depressed patients irrespective of the impact of depression on other chronic illnesses. 
The only study in literature which examined the impact of depression on nondepression related health care costs in type 2 diabetes patients was conducted by Finklestein and associates ${ }^{127}$ using claims data from the 1997 Medicare 5\% Standard Analytic Files. The study results indicated that controlling for age, gender, race, and comorbidities non-mental health care costs were approximately $21.00 \%$ higher for depressed diabetics as compared to non-depressed diabetics. The authors also concluded that depressed patients seek treatment for more services and when admitted, spend more time in inpatient facilities than patients without depression. These differences in terms of non-depression health costs between depressed and non-depressed diabetic patients were lower than those obtained in our study $(32.10 \%)$. Numbers in this study may be higher since a more comprehensive utilization data was available for the study population as opposed to Medicare claims which may not be furnish information related to some expenditures such as prescription costs. The cost differences may also be due to a younger population in this study (18-64 years of age) comprising of only newly diagnosed type 2 diabetes patients.

The findings of higher health care costs among depressed patients in this study corresponds with the literature that in patients with diverse medical illnesses, depression makes a significant independent contribution to increased health care costs. ${ }^{113}$ Studies have shown depression to be a good predictor of symptoms commonly associated with worsening glucose control such as polyphagia and polydipsia. Depressive symptoms may also be associated with a perception of impaired health and thus simulate worsening diabetes symptoms. ${ }^{119}$ Such perceptions on the part of the patient may prompt both the patient and provider to increase diagnostic testing, medical testing, and pursue aggressive 
treatment strategies. Adherence to medications and other behaviors can also be a mediator between depression and health care expenditures.

\section{$\underline{\text { Results for Objective } 9}$}

To examine the causal pathways between preexisting depression, adherence, and outcomes such as resource utilization and costs for type 2 diabetes

Earlier objectives have examined the role that depression can play in affecting adherence to oral hypoglycemics and type 2 diabetes related utilization and expenditures. The following 4 steps were used to identify whether adherence with oral hypoglycemics mediates the relationship between depression and type 2 diabetes outcomes. The mediating relation is going to be tested using the four steps mentioned in the methodology section.

Step 1: If depression is a significant predictor of type 2 diabetes outcomes Results of a multivariate Semi-log Ordinary Least Squares (OLS) model indicated that controlling for confounding covariates, patients with depression incurred $21.27 \%$ higher type 2 diabetes costs as compared to non-depressed patients in the 12 month follow up period (Table 19). These cost differences were primarily due to increased likelihood of ER/hospitalization episodes for depressed patients. Results from a multivariate logistic regression model indicated that depressed patients were nearly 1.4 times more likely to have an ER/hospitalization episode as compared to non-depressed patients (Table 20A). 
Step 2: If depression significantly predicts adherence with oral hypoglycemics

Results indicated that patients with depression had significantly lower adherence to oral hypoglycemics than non-depressed patients on the basis of both Reg MPR-1 and Reg MPR-2. However, although the differences were significant the magnitude of difference was not large with depressed patients being only $3.00 \%$ and $6.00 \%$ less adherent than non-depressed patients on the Reg MPR-1 and Reg MPR-2 index respectively. These estimates were found to be similar in both the univariate and multivariate framework (Tables 14 and 15).

The multinomial logistic regression model for the impact of depression on categories of adherence (Reg MPR-1 and Reg MPR-2) also indicated similar results. Results of the multivariate model for Reg MPR-1 indicated that controlling for confounding covariates, patients with depression were 1.9 times more likely to have low adherence to oral hypoglycemics as compared non-depressed patients (Table 16B). Results from the model for Reg MPR-2 demonstrated that depressed patients were 1.6 times more likely to be in the very low adherence group as compared to non-depressed patients (Table 17A).

Step 3: If adherence significantly predicts outcomes while controlling for depression. Results from the semi-log OLS models for the effect of adherence (Reg MPR-1 and Reg MPR-2) on type 2 diabetes related total costs were inconclusive. A non-linear pattern of the effect of adherence on costs was observed with results indicating that patients who had very low adherence have lower costs and patients with over adherence have higher costs than patients with good adherence. 
The semi-log OLS model for the impact of Reg MPR-1 on type 2 diabetes costs indicated that patients who were over adherent to their oral hypoglycemics medications incurred nearly $65.00 \%$ greater type 2 diabetes related costs than patients who were adherent to their medications $(\mathrm{p}=0.004)$ (Table 34). However, adherence with oral hypoglycemics was not associated with the probability of a type 2 diabetes related ER/hospitalization episode (Tables 35).

The semi-log OLS model estimating the impact of Reg MPR-2 on type 2 diabetes costs demonstrated that patients with very low adherence had nearly $57.00 \%$ lower costs as compared to patients who were adherent to their medications ( $\mathrm{p}=0.000)$ (Table 36$)$. The logistic regression model to estimate the impact of adherence (Reg MPR-2) on ER/hospitalization costs (Table 37) demonstrated that patients with low adherence were nearly 1.6 times more likely to have a diabetes related ER/hospitalization episode $(\mathrm{p}=0.029)$.

Step 4: If adherence mediates the impact of depression on type 2 diabetes outcomes To establish that adherence completely mediates the relationship between depression and outcomes, the effect of depression on outcomes controlling for adherence should be zero. If partial mediation is exhibited then the effect of depression on outcomes controlling for adherence should reduce in size. However, in both the models to estimate effect of adherence on type 2 diabetes costs, depression demonstrated a significant effect on outcomes without reducing its effect size as compared to models not controlling for the effect of adherence.

A multivariate model indicated that controlling for confounding covariates other than adherence, patients with depression incurred $21.27 \%$ higher type 2 diabetes costs as 
compared to non-depressed patients (Table 19). The impact of depression on type 2 diabetes costs remained significant even in models adjusted for adherence. The effect size of depression also remained similar to the original model with adherence-adjusted models indicating that patients with depression incurred 23\%-31\% higher costs than nondepressed patients (Table 34, 36).

Controlling for confounding covariates other than adherence, depressed patients were nearly 1.4 times more likely to have an ER/hospitalization episode as compared to non-depressed patients $(\mathrm{p}=0.028)$. (Table 20A) This effect of depression was maintained in adherence-adjusted models with odds ratio of 1.537 and 1.390 for Reg MPR-1 and Reg MPR-2 adjusted models respectively (Table 35, 37). The presence of mediation was statistically tested by comparing the estimated effects of depression on type 2 diabetes outcomes with and without controlling for adherence using a Hausman type statistic. This statistic displayed no significant differences between the estimates confirming the absence of adherence to oral hypoglycemic agents as a mediator between depression and type 2 diabetes outcomes. 
Table 34: Semi-log Ordinary Least Squares (OLS) regression model for the impact of adherence (MPR-1) on total diabetes related costs

$\begin{array}{llll}\text { Beta } & \text { S.E. } & \text { Test statistic } & \text { Sig. }\end{array}$

(

(p)

Depression

0.210

0.081

$2.5790 .010^{*}$

Adherence (Ref: Good adherence)

- Very low adherence

- Low adherence

- Average adherence

- Over adherence

$\begin{array}{rrrl}-0.199 & 0.248 & -0.801 & 0.423 \\ -0.164 & 0.129 & -1.263 & 0.207 \\ -0.024 & 0.087 & -0.277 & 0.782 \\ 0.520 & 0.181 & 2.870 & 0.004^{*}\end{array}$

Demographic characteristics

Age (in years)

$0.001 \quad 0.004$

$0.221 \quad 0.825$

Males (ref: females)

$0.024 \quad 0.075$

$0.324 \quad 0.746$

Whites (ref: non-whites)

$-0.027$

0.163

$-0.165 \quad 0.869$

Urban (ref: rural)

$-0.157$

0.128

$-1.225 \quad 0.221$

Year of index prescription (ref: 2001)

- 1998

- 1999

$-0.252$

0.190

$-1.325$

1.032

0.185

- 2000

0.184

0.178

$\begin{array}{ll}0.555 & 0.579\end{array}$

Co-morbid conditions in the pre period

Number of ER/hosp visits

0.059

0.167

0.303

0.093

0.019

$3.0680 .002 *$

Total health costs

$0.000 \quad 0.000$

$-2.0910 .037 *$

Charlson co-morbidity

$0.141 \quad 0.045$

$3.1270 .002 *$

Number of therapeutic classes

of medications

Cardio-vascular

$0.043 \quad 0.023$

$\begin{array}{ll}1.910 & 0.057\end{array}$

0.031

0.083

0.371

0.711

Cancer

$-0.078$

0.215

$-0.363 \quad 0.716$

Asthma

0.050

0.111

$\begin{array}{ll}0.453 & 0.651\end{array}$

Ulcers

0.352

0.231

$1.521 \quad 0.129$ 
Table 34: Semi-log Ordinary Least Squares (OLS) regression model for the impact of adherence (Reg MPR-1) on total diabetes related costs (Contd.)

\begin{tabular}{|c|c|c|c|c|}
\hline & Beta & S.E. & $\begin{array}{c}\text { Test statistic } \\
(t)\end{array}$ & $\begin{array}{l}\text { Sig. } \\
(p)\end{array}$ \\
\hline \multicolumn{5}{|l|}{ Provider interaction in the pre period } \\
\hline PAAS enrollment & 0.252 & 0.111 & 2.268 & $0.024 *$ \\
\hline \multicolumn{5}{|c|}{ Number of pharmacies visited (ref: multiple pharmacies) } \\
\hline - No pharmacy & -0.059 & 0.194 & -0.306 & 0.760 \\
\hline - Single pharmacy & -0.024 & 0.074 & -0.324 & 0.746 \\
\hline \multicolumn{5}{|l|}{ Diabetes severity } \\
\hline \multicolumn{5}{|c|}{ Index oral hypoglycemic prescription by: (ref: Family medicine) } \\
\hline - Endocrinologist & 0.292 & 0.274 & 1.066 & 0.287 \\
\hline - Internal medicine & 0.044 & 0.079 & 0.564 & 0.573 \\
\hline Total diabetes costs & 0.000 & 0.000 & 1.399 & 0.162 \\
\hline $\begin{array}{l}\text { Number of diabetes related } \\
\text { ER/Hosp visits }\end{array}$ & 0.125 & 0.104 & 1.203 & 0.229 \\
\hline $\begin{array}{l}\text { Gap between index diagnosis } \\
\text { and prescription }\end{array}$ & 0.000 & 0.000 & 0.467 & 0.641 \\
\hline Initial polytherapy & 0.565 & 0.140 & 4.030 & $0.000 *$ \\
\hline \multicolumn{5}{|c|}{ Index class of oral hypoglycemic (ref: Sulfonyl urea) } \\
\hline - Thiazolidenedione & 0.553 & 0.151 & 3.665 & $0.000 *$ \\
\hline - Biguanides & 0.279 & 0.079 & 3.534 & $0.000 *$ \\
\hline - Alpha-glucosidase & 0.177 & 0.282 & 0.627 & 0.531 \\
\hline - Sulfonyl-biguanides & -0.643 & 0.248 & -2.597 & $0.010^{*}$ \\
\hline - Meglitinides & 0.676 & 0.285 & 2.370 & $0.018 *$ \\
\hline Constant & 6.646 & 0.339 & 19.602 & 0.000 \\
\hline
\end{tabular}

*significance at the 0.05 level

PAAS: Physician Assured Access System

Model fit statistics:

R-square: $20.8 \% ; \mathrm{F}=6.422 ; \mathrm{P}=0.000$

Durbin-Watson: 2.050

White test for heteroskedasticity: $\chi^{2}=465.86 ; p=0.7675$ 
Table 35: Logistic regression for the impact of adherence (Reg MPR-1) on having a type 2 diabetes related ER/hospitalization episode

\begin{tabular}{lcccccrc}
\hline & Beta & S.E. Sig. & $\begin{array}{c}\text { Odds } \\
\text { ratio }\end{array}$ & $\begin{array}{r}\text { 95\% C.I. for } \\
\text { Odds ratio } \\
\text { Lower }\end{array}$ \\
\hline Depression & & & (p) & & & &
\end{tabular}

Adherence (Ref: Good adherence)

- Very low adherence

- Low adherence

- Average adherence

- Over adherence $\begin{array}{lll}0.566 & 0.555 & 0.307\end{array}$

$\begin{array}{lll}0.137 & 0.305 & 0.653\end{array}$

$\begin{array}{lll}0.266 & 0.208 & 0.202\end{array}$

$\begin{array}{lll}0.378 & 0.415 & 0.362\end{array}$

Demographic characteristics

Age (in years)

$\begin{array}{lll}0.006 & 0.010 & 0.520\end{array}$

$\begin{array}{lll}-0.125 & 0.185 & 0.499\end{array}$

$\begin{array}{lll}0.141 & 0.420 & 0.738\end{array}$

$\begin{array}{lll}-0.033 & 0.310 & 0.915\end{array}$

Urban (ref: rural)

Males (ref: females)

Year of index prescription (ref: 2001)

\begin{tabular}{|c|c|c|c|c|c|}
\hline 1998 & -0.513 & 0.46 & 0.266 & 0.599 & 0.243 \\
\hline 199 & 0.261 & 0.42 & 0.539 & 1.298 & 0.565 \\
\hline 2000 & 0.049 & 0.39 & 0.902 & 1.050 & 0.481 \\
\hline
\end{tabular}

Co-morbid conditions in the pre period

Number of ER/hosp visits

0.276

$0.055 \quad 0.000 *$

1.318

$1.183 \quad 1.468$

Total health costs

$\begin{array}{lll}0.000 & 0.000 & 0.971\end{array}$

$\begin{array}{lll}1.000 & 1.000 \quad 1.000\end{array}$

Charlson co-morbidity

$\begin{array}{lll}0.161 & 0.105 & 0.126\end{array}$

1.175

$0.956 \quad 1.445$

Number of therapeutic classes of medications

Cardio-vascular

Cancer

Asthma

Ulcers

$\begin{array}{cccccc}0.106 & 0.053 & 0.043 * & 1.112 & 1.003 & 1.233 \\ 0.009 & 0.203 & 0.965 & 1.009 & 0.678 & 1.501 \\ -0.623 & 0.525 & 0.236 & 0.536 & 0.191 & 1.502 \\ 0.376 & 0.247 & 0.127 & 1.457 & 0.898 & 2.364 \\ 1.125 & 0.529 & 0.034^{*} & 3.081 & 1.091 & 8.697\end{array}$


Table 35: Logistic regression for the impact of adherence (Reg MPR-1) on having a type 2 diabetes related ER/Hospitalization episode

\begin{tabular}{|c|c|c|c|c|c|c|}
\hline & \multirow[t]{2}{*}{ Beta } & \multirow[t]{2}{*}{ S.E. } & \multirow[t]{2}{*}{$\begin{array}{l}\text { Sig. } \\
(p)\end{array}$} & \multirow[t]{2}{*}{$\begin{array}{l}\text { Odds } \\
\text { ratio }\end{array}$} & \multicolumn{2}{|c|}{$\begin{array}{r}\text { 95\% C.I. for } \\
\text { Odds ratio }\end{array}$} \\
\hline & & & & & Lower & Upper \\
\hline \multicolumn{7}{|c|}{ Provider interaction in the pre period } \\
\hline PAAS enrollment & 0.114 & 0.265 & 0.666 & 1.121 & 0.667 & 1.883 \\
\hline \multicolumn{7}{|c|}{ Number of pharmacies visited (ref: multiple pharmacies) } \\
\hline - No pharmacy & -0.037 & 0.550 & 0.946 & 0.963 & 0.328 & 2.829 \\
\hline - Single pharmacy & 0.170 & 0.179 & 0.343 & 1.185 & 0.834 & 1.683 \\
\hline
\end{tabular}

\section{Diabetes severity}

Index oral hypoglycemic prescription by: (ref: Family medicine)

- Endocrinologist

- Internal medicine

Total diabetes costs

Number of diabetes related

$\mathrm{ER} /$ Hosp visits

Gap between index diagnosis and prescription

Initial polytherapy $\begin{array}{lll}0.341 & 0.610 & 0.577\end{array}$

$\begin{array}{lll}0.178 & 0.185 & 0.334\end{array}$

$\begin{array}{lll}0.000 & 0.000 & 0.978\end{array}$

$0.409 \quad 0.241 \quad 0.090$

$\begin{array}{lll}0.000 & 0.000 & 0.825\end{array}$

$\begin{array}{lll}0.249 & 0.321 & 0.438\end{array}$

Index class of oral hypoglycemic (ref: Sulfonyl urea)

- Thiazolidenedione

- Biguanides

$-0.158$

0.128

0.365

0.666

$\begin{array}{lll}-1.008 & 0.871 & 0.247\end{array}$

$\begin{array}{lll}-0.484 & 0.602 & 0.422\end{array}$

$\begin{array}{lll}0.420 & 0.630 & 0.505\end{array}$

- Meglitinides

Constant $\begin{array}{lll}1.406 & 0.425 & 4.646\end{array}$

$\begin{array}{lll}1.195 & 0.832 \quad 1.716\end{array}$

$1.000 \quad 1.000 \quad 1.000$

$\begin{array}{lll}1.505 & 0.938 & 2.414\end{array}$

$\begin{array}{lll}1.000 & 0.999 \quad 1.001\end{array}$

$\begin{array}{lll}1.283 & 0.683 & 2.408\end{array}$

$\begin{array}{lll}0.854 & 0.418 & 1.746\end{array}$

$\begin{array}{lll}1.137 & 0.784 & 1.648\end{array}$

$\begin{array}{lll}0.365 & 0.066 & 2.012\end{array}$

$\begin{array}{lll}0.616 & 0.189 & 2.006\end{array}$

$\begin{array}{lll}1.521 & 0.443 \quad 5.230\end{array}$

*Significance at the 0.05 level

PAAS: Physician Assured Access System

\section{Model fit statistics:}

Pseudo R-square $=23.5 \%$

-2 Log Likelihood $=871.069 ; \chi^{2}=153.705, \mathrm{p}=0.000$

Hosmer and Lemeshow's goodness of fit: $\chi^{2}=8.552 ; p=0.381$ 
Table 36: Semi-log Ordinary Least Squares (OLS) regression model for the impact of adherence (Reg MPR-2) on total diabetes related costs

Beta S.E. Test statistic Sig.

(t)

(p)

\section{Depression}

Adherence (Ref: Good adherence)

- Very low adherence

- Low adherence

- Average adherence

- Over adherence

0.275

$-0.840$

0.023

$-0.061$

0.166
Demographic characteristics

Age (in years)

Males (ref: females)

Whites (ref: non-whites)

Urban (ref: rural)

Year of index prescription (ref: 2001)

- 1998

- 1999

- 2000

0.003

0.056

0.066

$-0.197$

$-0.014$

0.393

0.306
Co-morbid conditions in the pre period Number of ER/hosp visits

Total health costs

Charlson co-morbidity

Number of therapeutic classes

of medications

Cardio-vascular

Cancer

Asthma

Ulcers

$\begin{array}{llll}0.072 & 0.014 & 5.206 & 0.000^{*}\end{array}$

$\begin{array}{llll}0.000 & 0.000 & -2.343 & 0.019 *\end{array}$

$0.137 \quad 0.040$

$3.4380 .001 *$

$0.053 \quad 0.021$

$2.4890 .013^{*}$

$0.026 \quad 0.076$

$0.339 \quad 0.735$

$-0.337$

0.188

$\begin{array}{ll}-1.797 & 0.073\end{array}$

0.052

0.102

$0.513 \quad 0.608$

$0.235 \quad 0.202$

$1.165 \quad 0.244$ 
Table 36: Semi-log Ordinary Least Squares (OLS) regression model for the impact of adherence (Reg MPR-2) on total diabetes related costs (Contd.)

Beta S.E. Test statistic Sig.

(t) (p)

Provider interaction in the pre period

$\begin{array}{lllll}\text { PAAS enrollment } & 0.128 & 0.104 & 1.235 & 0.217\end{array}$

Number of pharmacies visited (ref: multiple pharmacies)
- No pharmacy
0.031
0.171
$\begin{array}{ll}0.182 & 0.855\end{array}$
- Single pharmacy
$-0.034$
0.070
$-0.484 \quad 0.628$

Diabetes severity

Index oral hypoglycemic prescription by: (ref: Family medicine)
- Endocrinologist
$\begin{array}{ll}0.307 & 0.270\end{array}$
$\begin{array}{ll}1.137 & 0.256\end{array}$
- Internal medicine
0.057
0.073
$0.781 \quad 0.435$

Total diabetes costs

$0.000 \quad 0.000$

$0.467 \quad 0.641$

Number of diabetes related

$0.217 \quad 0.099$

$2.2050 .028 *$

ER/Hosp visits

Gap between index diagnosis

$0.000 \quad 0.000$

$1.309 \quad 0.191$

and prescription

Initial polytherapy

0.665

0.148

$4.4960 .000^{*}$

Index class of oral hypoglycemic (ref: Sulfonyl urea)
- Thiazolidenedione
0.612
0.142
$4.3120 .000^{*}$
- Biguanides
0.192
0.074
$2.593 \quad 0.010 *$
- Alpha-glucosidase
$-0.073$
0.271
$-0.2690 .788$
- Sulfonyl-biguanides
$-0.735$
0.252
$-2.9140 .004 *$
- Meglitinides
0.506
0.265
1.9140 .056

Constant

6.259

0.316

$19.794 \quad 0.000$

*significance at the 0.05 level

PAAS: Physician Assured Access System

\section{Model fit statistics:}

R-square: $23.7 \% ; \mathrm{F}=11.479 ; \mathrm{P}=0.000$

Durbin-Watson: 1.995

White test for heteroskedasticity: $\chi^{2}=596.11 ; p=0.0678$ 
Table 37: Logistic regression for the impact of adherence (Reg MPR-2) on having a type 2 diabetes related ER/hospitalization episode

\begin{tabular}{lccccccc}
\hline & Beta & S.E. & $\begin{array}{l}\text { Sig. } \\
(p)\end{array}$ & $\begin{array}{l}\text { Odds } \\
\text { ratio }\end{array}$ & $\begin{array}{r}\text { 95\% C.I. for } \\
\text { Odds ratio } \\
\text { Lower Upper }\end{array}$ \\
\hline Depression & & & & & & & Up
\end{tabular}

Adherence (Ref: Good adherence)

- Very low adherence

$\begin{array}{rlllll}-0.025 & 0.175 & 0.885 & 0.975 & 0.692 & 1.374 \\ 0.485 & 0.222 & 0.029 * & 1.624 & 1.051 & 2.510 \\ -0.070 & 0.217 & 0.748 & 0.932 & 0.609 & 1.428 \\ 0.037 & 0.267 & 0.890 & 1.038 & 0.615 & 1.752\end{array}$

- Low adherence

- Average adherence

$\begin{array}{lll}0.037 & 0.267 & 0.890\end{array}$

1.038

$0.615 \quad 1.752$

Demographic characteristics

Age (in years)

$\begin{array}{llllll}0.002 & 0.007 & 0.804 & 1.002 & 0.987 & 1.016\end{array}$

Males (ref: females)

$\begin{array}{lll}-0.038 & 0.150 & 0.802\end{array}$

$0.963 \quad 0.718 \quad 1.292$

Whites (ref: non-whites)

$\begin{array}{lll}-0.013 & 0.311 & 0.965\end{array}$

$0.987 \quad 0.536 \quad 1.815$

Urban (ref: rural)

$\begin{array}{lll}0.095 & 0.258 & 0.713\end{array}$

$\begin{array}{lll}1.100 & 0.663 \quad 1.824\end{array}$

Year of index prescription (ref: 2001)
- 1998
$-0.287$
$0.372 \quad 0.439$
0.750
0.362
1.554
- 1999
$\begin{array}{lll}0.419 & 0.344 & 0.223\end{array}$
1.520
$0.775 \quad 2.983$
- 2000
$\begin{array}{lll}0.320 & 0.324 & 0.323\end{array}$
1.377
$0.730 \quad 2.598$

Co-morbid conditions in the pre period

Number of ER/hosp visits

0.232

$0.0380 .000 *$

$1.261 \quad 1.170 \quad 1.359$

Total health costs

0.000

$0.000 \quad 0.493$

$1.000 \quad 1.000 \quad 1.000$

Charlson co-morbidity

$\begin{array}{lll}0.143 & 0.077 & 0.065\end{array}$

1.154

0.991

1.343

Number of therapeutic classes of medications

Cardio-vascular

$0.104 \quad 0.042 \quad 0.014^{*}$

$1.110 \quad 1.021 \quad 1.206$

$\begin{array}{lll}-0.034 & 0.157 & 0.831\end{array}$

0.967

$0.711 \quad 1.316$

Cancer

$\begin{array}{lll}-0.483 & 0.388 & 0.213\end{array}$

0.617

$0.288 \quad 1.319$

Asthma

$\begin{array}{lll}0.238 & 0.194 & 0.220\end{array}$

$\begin{array}{lll}1.268 & 0.868 \quad 1.854\end{array}$

Ulcers

$\begin{array}{lll}0.663 & 0.394 & 0.092\end{array}$

$1.941 \quad 0.898$

4.199 
Table 37: Logistic regression for the impact of adherence (Reg MPR-2) on having a type 2 diabetes related ER/hospitalization episode (Contd.)

\begin{tabular}{|c|c|c|c|c|c|c|}
\hline & \multirow[t]{2}{*}{ Beta } & \multirow[t]{2}{*}{ S.E. } & \multirow[t]{2}{*}{$\begin{array}{l}\text { Sig. } \\
\text { (p) }\end{array}$} & \multirow[t]{2}{*}{$\begin{array}{l}\text { Odds } \\
\text { ratio }\end{array}$} & \multicolumn{2}{|c|}{$\begin{array}{r}95 \% \text { C.I. for } \\
\text { Odds ratio }\end{array}$} \\
\hline & & & & & Lower & Upper \\
\hline \multicolumn{7}{|c|}{ Provider interaction in the pre period } \\
\hline PAAS enrollment & -0.047 & 0.216 & 0.828 & 0.954 & 0.625 & 1.457 \\
\hline \multicolumn{7}{|c|}{ Number of pharmacies visited (ref: multiple pharmacies) } \\
\hline - No pharmacy & 0.197 & 0.376 & 0.601 & 1.217 & 0.583 & 2.541 \\
\hline - Single pharmacy & 0.063 & 0.145 & 0.664 & 1.065 & 0.802 & 1.413 \\
\hline
\end{tabular}

\section{Diabetes severity}

Index oral hypoglycemic prescription by: (ref: Family medicine)
- Endocrinologist
$\begin{array}{lll}0.370 & 0.519 & 0.476\end{array}$
$\begin{array}{lll}1.447 & 0.523 & 4.002\end{array}$
- Internal medicine
$\begin{array}{lll}0.139 & 0.147 & 0.344\end{array}$
1.149
0.862
1.532

Total diabetes costs

$\begin{array}{lll}0.000 & 0.000 & 0.720\end{array}$

$1.000 \quad 1.000 \quad 1.000$

Number of diabetes related

$0.455 \quad 0.199 \quad 0.022 *$

$1.577 \quad 1.067$

2.329

ER/Hosp visits

$\begin{array}{lrrrrrr}\begin{array}{l}\text { Gap between index diagnosis } \\ \text { and prescription }\end{array} & 0.000 & 0.000 & 0.955 & 1.000 & 0.999 & 1.001 \\ & & & & & & \\ \text { Initial polytherapy } & 0.213 & 0.290 & 0.463 & 1.237 & 0.701 & 2.184 \\ & & & & & & \\ \text { Index class of oral hypoglycemic (ref: Sulfonyl urea) } & & & & \\ \text { - Thiazolidenedione } & -0.006 & 0.280 & 0.983 & 0.994 & 0.574 & 1.722 \\ \text { - Biguanides } & -0.085 & 0.152 & 0.577 & 0.918 & 0.681 & 1.238 \\ \text { - Alpha-glucosidase } & -0.918 & 0.672 & 0.172 & 0.399 & 0.107 & 1.491 \\ \text { - Sulfonylurea-biguanide } & -0.572 & 0.520 & 0.271 & 0.564 & 0.204 & 1.563 \\ \text { - Meglitinides } & -0.078 & 0.546 & 0.887 & 0.925 & 0.317 & 2.698 \\ \text { Constant } & -2.266 & 0.658 & 0.001 & 0.104 & & \end{array}$

*significance at the 0.05 level

PAAS: Physician Assured Access System

\section{Model fit statistics:}

Pseudo $R$-square $=20.2 \%$

-2 Log Likelihood $=1344.536 ; \chi^{2}=195.596, p=0.000$

Hosmer and Lemeshow's goodness of fit: $\chi^{2}=4.848 ; p=0.774$ 


\section{Discussion for Objective 9}

The four step method for determining mediation indicated that adherence to oral hypoglycemics is not a mediator in the relationship between depression and type 2 diabetes. Lustman and associates ${ }^{122,123}$ and Gary and associates ${ }^{131}$ conducted studies using different methodological approaches to determine the mediating role of adherence in the impact of depression on diabetes outcomes. Their findings were similar to our study results with depression affecting diabetes outcomes without adherence being a mediating variable.

As for the effect of adherence on type 2 diabetes related costs, our results indicated lack of a strong association. However, the effect was non-linear with results indicating that patients who had very low adherence have lower costs and patients with over adherence have higher costs than patients with good adherence. For the middle group, there seemed to be a linear trend with improving adherence decreasing type 2 diabetes costs. An interesting finding is the effect of over adherence on costs. Over adherence is ignored in a lot of adherence studies by truncating the adherence levels at 1.0. In some situations over adherence may not be serious, while in other situations it may result in serious conditions such as hypoglycemia or may be indicative of over aggressive pharmacotherapy. The study also examined the effect of over adherence separately on non-prescription type 2 diabetes costs. The effects remained the same indicating higher type 2 diabetes costs in patients who are over adherent to their oral hypoglycemics. Low costs for patients with low adherence may possibly be due to the fact that these patients have achieved glycemic control through diet and exercise and hence did not regularly filled their prescriptions. These results were similar to those of a 
recent retrospective cohort study conducted in a non-managed care setting, wherein a non-linear effect was observed with significantly lower costs for patients with less than $20 \%$ adherence and significantly higher costs for patients with more than $100 \%$ adherence. Adherence levels somewhere in between showed a weak negative linear association with type 2 diabetes costs. ${ }^{84}$

The study had a short follow up period of one year. Diabetes is a chronic illness and complications of diabetes normally arise years after diagnosis. Improved adherence with oral hypoglycemics is likely to increase pharmaceutical costs in the short term. The decreases in medical costs due to improved adherence may not be enough to offset the high costs of pharmaceuticals in the short term. However, a couple of sub-analyses were conducted to explore these issues. The objectives were examined in a sub-sample with a 24-month follow up period. Also, the impact of adherence on type 2 diabetes related costs excluding those due to pharmaceutical expenditures were examined. However, these sub-analyses provided identical results. It may take many years of improved glycemic control through adherence to oral hypoglycemics to reduce medical costs associated with treatment of long-term complications. Thus, the relationship between depression, adherence, and type 2 diabetes related expenditures might differ with a longer measurement period.

These results of this study could be possible as one failed to measure adherence to other aspects of diabetes therapy such as diet, exercise, or glucose monitoring. Also, the relation between depression and type 2 diabetes outcomes might occur via physiological pathways that are independent of behavioral factors. It is possible that a common neuroendocrine factor leads to both depression and diabetes. The increased expenditures 
may be a direct result of worsening glucose control due to changes in the autonomic nervous system, the hypothalamic-pituitary adrenal axis, or neurotransmitters. 


\section{CHAPTER FIVE}

\section{SUMMARY AND CONCLUSIONS}

This chapter provides and overview of the study findings, draws conclusions, presents implications of the study, lists limitations, and provides recommendations for future research.

\section{Phase 1}

The potential effects of depression on outcomes associated with management of type 2 diabetes may be significant when one considers that depression is highly prevalent in patients with diabetes. In addition to the increased prevalence of co-morbid depression in patients with type 2 diabetes, a few studies have also indicated an increased incidence of type 2 diabetes in patients with depression. Depression is related to changes in nutrition and exercise that may contribute to risk for diabetes; and there may be somatic aspects of depression such as a change in the immune or vascular system that contributes to enhanced risk for diabetes onset. Phase 1 of the study examined the epidemiological relationship between depression and type 2 diabetes. Objectives of this phase included determining the prevalence of co-morbid depression in patients with type 2 diabetes and the incidence of type 2 diabetes in patients with pre-existing depression. 


\section{Conclusions for Phase 1}

Objective 1: To estimate prevalence of co-morbid depression in patients with type 2 diabetes

The null hypothesis for this objective was that there is no difference between the prevalence of co-morbid depression in patients with type 2 diabetes and without type 2 diabetes. The study results indicated that co-morbid depression was more prevalent in patients with type 2 diabetes as compared to enrollees without type 2 diabetes. The higher prevalence of depression was primarily due to the higher number of co-morbid conditions associated with type 2 diabetes. However, one can attribute these co-morbid conditions and subsequent depression to type 2 diabetes and thus reasonably conclude that type 2 diabetes is associated with a higher prevalence of co-morbid depression.

Objective 2: To estimate the incidence of type 2 diabetes in patients with pre-existing depression

The null hypothesis for this objective was that there is no difference in the incidence rate of type 2 diabetes in patients with pre-existing depression as compared to patients without pre-existing depression. The results indicated that female patients with pre-existing depression are more likely to develop type 2 diabetes as compared to enrollees without pre-existing depression controlling for baseline confounding factors such as demographics and co-morbidity. No differences in incident rates were observed in males. 


\section{Phase 2}

Phase 2 of the study examined the impact of depression on patterns of oral hypoglycemic use, initiation of insulin therapy, and adherence with oral hypoglycemics. The effect of depression on utilization and expenditures related to type 2 diabetes and overall health care (excluding those due to depressive diagnosis) was also estimated. In addition, the study also tested the mediating influence of adherence with oral hypoglycemics between depression and outcomes related to management of type 2 diabetes.

\section{Conclusions for Phase 2}

Objective 3: To examine the impact of preexisting depression on patterns of oral hypoglycemic use in new patients with type 2 diabetes

The null hypothesis for this objective was that there is no difference in the pattern of oral hypoglycemic use between newly diagnosed type 2 diabetes patients who have pre-existing depression and those who do not have pre-existing depression. The study findings indicated that patients without depression had a significantly favorable pattern of oral hypoglycemic use as compared to non-depressed patients. A significantly higher proportion of depressed patients, switched, augmented or discontinued their index oral hypoglycemic therapy as compared to non-depressed patients. 
Objective 4: To examine the impact of preexisting depression on modification of oral hypoglycemic therapy

The null hypothesis for this objective was that there is no difference in the rate of modification of oral hypoglycemics between newly diagnosed type 2 diabetes patients who have pre-existing depression and those who do not have pre-existing depression. The results indicated that patients with depression had a significantly higher rate of modification of oral hypoglycemic therapy and had a relatively shorter time to modification as compared to non-depressed patients.

Objective 5: To estimate the impact of preexisting depression on initiation of insulin therapy in patients with type 2 diabetes

The null hypothesis for this objective was that there is no difference in the rate of initiation of insulin therapy between newly diagnosed type 2 diabetes patients who have pre-existing depression and those who do not have pre-existing depression. The null hypothesis for this objective was accepted as no differences were seen in rates of insulin initiation and time to insulin therapy between depressed and non-depressed patients in the 12-month follow up period.

Objective 6: To estimate the impact of preexisting depression on adherence to oral hypoglycemic agents

The null hypothesis for this objective was that there is no difference in adherence to oral hypoglycemics between newly diagnosed type 2 diabetes patients who have preexisting depression and those who do not have pre-existing depression. The findings 
demonstrated that depressed patients had significantly lower adherence to oral hypoglycemic agents as compared to non-depressed patients. However, the magnitude of difference was not large with results indicating that patients with depression were 3-6\% less adherent than non-depressed patients.

Objective 7: To estimate the impact of preexisting depression on type 2 diabetes related utilization and costs

The null hypothesis for this objective was that there is no difference in type 2 diabetes related utilization and costs between newly diagnosed type 2 diabetes patients who have pre-existing depression and those who do not have pre-existing depression. Patients with depression had significantly higher type 2 diabetes related costs as compared to non-depressed patients. Results indicated that controlling for confounding covariates, patients with depression incurred around $21.30 \%$ higher type 2 diabetes costs as compared to non-depressed patients. These cost differences were primarily due to a higher probability of an ER/hospitalization episode in depressed patients as compared to non-depressed patients. Also, a significant difference between the two groups was in terms of number of type 2 diabetes related ER/hospitalizations with depressed patients incurring nearly $57.80 \%$ higher number of visits as compared to non depressed patients.

Objective 8: To estimate the impact of preexisting depression on overall health care utilization and costs (excluding those due to depressive diagnosis)

The null hypothesis for this objective was that there is no difference in overall health care utilization and costs between newly diagnosed type 2 diabetes patients who 
have pre-existing depression and those who do not have pre-existing depression. Patients with depression had nearly $32.10 \%$ higher overall health care costs (excluding those due to depressive diagnosis) as compared to patients without depression. Depression was associated with increased costs in all areas of health care such as ER/hospitalization, outpatient, and prescription costs. A similar increase in health care utilization was observed for depressed patients in terms of number of ER/hospitalization episodes, physician office visits, and prescriptions in the 12-month follow up period.

Objective 9: To examine the causal pathways between preexisting depression, adherence, and outcomes such as resource utilization and costs for type 2 diabetes

The null hypothesis for this objective was that adherence to oral hypoglycemics is not a mediating variable between the presence of pre-existing depression and outcomes related to management of type 2 diabetes. Mediation analysis indicated that depression had a significant impact on both, type 2 diabetes related expenditures and adherence with oral hypoglycemics. However, adherence to oral hypoglycemics was not a mediator between depression and type 2 diabetes related expenditures. Depression could have impacted type 2 diabetes related outcomes directly through a physiological effect on glycemic levels or indirectly through its impact on adherence to other behaviors such as diet or exercise.

\section{Limitations}

Although our database study revealed the patterns of oral hypoglycemic use such as switching, augmentation, discontinuation, it gives no indication of the reasons for these patterns. These patterns could be a result of variety of reasons such as side effects, 
lack of efficacy, adequate glycemic control, or co-morbid conditions. Determining these reasons from claims data without additional clinical or patient/physician reported information is not possible. The study could have classified some patients who achieved glycemic control through diet and exercise and were taken off their oral hypoglycemic prescriptions as discontinuers and non-adherent. Also, the adherence measures were based on refill information from prescription claims data. Hence, the study makes the assumption that the prescriptions filled by patients were used appropriately. These indirect measures of adherence from claims data may not be considered the most accurate and reliable, but several studies have found significant associations between adherence measured from refill information and other methods such as self-report, pill count, medication diary. ${ }^{166}$

Adherence to medications can be affected by a number of factors, which were not measured in this study such as social support, perceived risk of the outcomes of being non-adherent, and others. One of the primary factors affecting adherence to oral hypoglycemics is diabetes severity. All our estimation models were controlled for severity of diabetes, which was measured from the information available from claims data. Although large variations in diabetes severity were not expected in this study population of newly diagnosed type 2 diabetes patients, additional laboratory values such as $\mathrm{A} 1 \mathrm{C}$ and blood glucose levels would have been helpful in determining disease severity. These laboratory values could also have been very useful as clinical outcome measures of diabetes therapy in addition to economic outcomes such as type 2 diabetes related expenditures. 
One of the primary limitations of this study is the absence of measurement of depression severity though a structured diagnostic interview. Depressed patients in this study are identified on the basis of seeking treatment for depression. Hence, it is possible that patients in this study classified as non-depressed may have depression symptomatology but not been diagnosed with depression. This issue of undiagnosed/unrecognized depression can potentially lead to misclassification bias as patient classified as non-depressed could be patients with undiagnosed depression. However, one can assume that diagnosed patients will have more severe depressive symptoms as compared to an undiagnosed population. Also, this misclassification could only lead to underestimation of the effect of depression on adherence to medications and related outcomes. The significant impact of depression found in this study's results would remain significant, but have a bigger magnitude of effect in absence of this misclassification.

The mediation analyses indicated that adherence to oral hypoglycemics had a non-linear and insignificant impact on type 2 diabetes related costs. However, diabetes is a chronic illness and complications of diabetes arise after several years of diagnosis. Improved adherence with oral hypoglycemics is likely to increase pharmaceutical costs in the short term. The decreases in medical costs due to improved adherence may not be enough to offset the high costs of pharmaceuticals in the short term. This study used a 12-month follow up period since the initial diagnosis of type 2 diabetes. It may take many years of improved glycemic control through adherence to oral hypoglycemics to reduce medical costs associated with treatment of long-term complications. Thus the 
relationship between depression, adherence, and type 2 diabetes related expenditures might differ with a longer measurement period.

\section{Implications}

Study results indicated that depression is associated with significantly higher type 2 diabetes and overall health care costs. Also, the rate of occurrence of depression in patients with type 2 diabetes was significantly higher as compared to Medicaid enrollees without type 2 diabetes. These results should encourage West Virginia Medicaid, national credentialing committees, large insurers, and employers to screen for depression in patients with type 2 diabetes and improve clinical management of depression in these patients. The implications of untreated depression on long-term type 2 diabetes complications can be even more significant when one considers that depression may be recurrent in diabetes patients. This data suggests that interventions are needed to screen diabetes patients for depression and use effective and aggressive therapies for treatment of depression. These data may provide an impetus for improving the detection and treatment of depression. Such increased effort in detecting and treating depression may be associated with additional non-psychiatric health benefits due to improved adherence, glycemic control, and decreased health care utilization.

Also, the observed higher risk of type 2 diabetes among female patients with depression may suggest that physicians need to monitor diabetic metabolic changes in females with depression for early detection and treatment of type 2 diabetes. Undetected and untreated type 2 diabetes can aggravate diabetic complications thus resulting in increased costs associated with management of type 2 diabetes. Considering the high 
prevalence of depression in West Virginia Medicaid, an improved screening and detection of type 2 diabetes may result in better disease management and cost saving. In addition to improving depression screening for patients with type 2 diabetes, one must systematically identify patients who have both type 2 diabetes and depression to ensure appropriate quality of care and the need for more aggressive treatment. The recognition of depression as a risk factor for decreasing adherence with oral hypoglycemics and increasing type 2 diabetes related expenditures has the potential to improve diabetes management and related outcomes. A potential barrier to effective depression management among type 2 diabetes patients is the lack of an overall unified view of a patient's condition encompassing both the conditions. Although the Health Plan Employer Data and Information Set (HEDIS) has included performance measures for both depression and diabetes care, separate standards should also be set for the treatment of type 2 diabetes with co-morbid depression. Programs addressing such multiple disorders must search for commonalities in treatment. ${ }^{167}$ A good example can be the emphasis on increased physical activity for management of both diabetes and depression. Also, given the crucial role of depression in diabetes, mental health professionals can play an important role in the care of these patients. This also calls for mental health professionals to create working relations with diabetes health care providers, including physicians, nurses, dietitians, pharmacists, and others.

Physicians can help improve patient adherence to treatment regimens by addressing adherence issues with their patients regularly and providing them with educational materials. Pharmacists can also assume a proactive role, especially in type 2 diabetes patients with co-morbid depression. These data suggest that patients with 
depressive symptoms should have their adherence to oral hypoglycemics monitored particularly closely and may require special encouragement from health care providers. Recognizing that a patient might be depressed could help a physician fathom the reasons for a patient's non-adherence and thus improve patient-physician relationship. For patients initiating treatment for type 2 diabetes, presence of depression might prove to be a useful indicator of possible future non-adherence and might suggest closer monitoring and assistance to achieve adherence to treatment. Alternatively, a lack of adherence with diabetes pharmacotherapy should cause concern for a possible presence of co-morbid depression.

\section{Directions for Future Research}

One of the main limitations of this study was the lack of clinical information regarding glycemic control and depression severity. The study also failed to measure adherence to other behaviors such as diet, exercise, and glucose monitoring that can affect management of type 2 diabetes. Incorporation of this clinical and adherence information in the study design can increase the validity of the findings. The relationship between depression and type 2 diabetes can be better explained in such a large scale longitudinal study that measure indicators such as adherence to medications, diet, and exercise, alterations in neurohormonal levels, depression severity, and glycemic levels at several points in time. Similar research can be conducted in patients with type 2 diabetes developing depression over the course of their diabetes management. The effect of the onset of depression on changing adherence with oral hypoglycemics and related outcomes in a prevalent type 2 diabetes patient should also be examined. 
There are a number of approaches to measure adherence with medications. Refill information using claims data seems a relatively valid and non-intrusive method to measure adherence. However, future studies can use multiple methods of adherence measurement such as pill count, self-report, or MEMS cap to validate the results from refill information. One of the main problems with refill data is the absence of information on free samples provided by physicians, which can especially invalidate the patterns of oral hypoglycemic use computed in our study. Similar studies in literature have measured patterns of use in terms of discontinuation, augmentation, modification, and switching from claims data. Future research needs to examine the extent of free samples being provided by physicians and adjustments be made to findings from claims data.

The excess costs attributable to depression in patients with type 2 diabetes or other chronic illness may be more significant if costs due to sick leave, missed productivity, or absenteeism from the work force are accounted. Thus, the impact of depression may more substantial from a societal perspective, which includes costs outside of the health care system. In future research, the policy discussion related to the impact of depression on costs should be expanded to include such indirect costs. 


\section{BIBLIOGRAPHY}

1. American Diabetes Association: Economic costs of diabetes mellitus in the US in 2002. Diabetes Care 2003; 26: 917-932

2. Maryland Department of Health and Mental Hygiene. Identifying a population at risk: Diabetes within the Maryland Medicaid population. Medicaid management short reports. Baltimore, MD: Department of Heath and Mental Hygiene; 1990

3. Harris MI, Flegal KM, Cowie CC, Eberhardt MS, Goldstein DE, Little RR, Wiedmeyer H, Byrd-Holt DD. Prevalence of diabetes, impaired fasting glucose, and impaired glucose tolerance in US adults. Diabetes Care 1998; 21:518-524

4. Rubin RJ, Altman WM, Mendelson DM. Healthcare expenditures for people with diabetes mellitus, 1992. J Clin Endocrin Met 1994; 78: 809A-809F

5. UKPDS Group. Intensive blood glucose control with sulphonylureas or insulin compared with conventional treatment and risk of complications in patients with type-2 diabetes (UKPDS 33). Lancet 1998; 352: 837-853

6. American Diabetes Association. Standards of medical care for patients with diabetes mellitus. Diabetes Care 2001; 24 (Suppl 1): S33-S43

7. Wisdom K, Fryzek JP, Havstad SL, Anderson RM, Dreiling MC, Tilley BC. Comparison of laboratory test frequency and test results between African Americans and Caucasians with diabetes. Opportunity for improvement. Diabetes Care 1997; 20: 971-977

8. Diehl AK, Bauer RL, Sugarek NJ. Correlates of medication compliance in noninsulin dependent diabetes mellitus. South Med J 1987; 80:332-335 
9. Chousa FP, Guillen VFG, Otero MD, Beltran DO, Lopez RP, Sanchez JM. Usefulness of six indirect methods to evaluate drug therapy compliance in noninsulin dependent diabetes mellitus. Revista Clinica Espanola 1997; 197:555-559

10. Peterson GM, McLean S, Senator GB. Determinants of patient compliance, control, presence of complications, and handicap in non-insulin dependent diabetes. Aust N Z J Med 1984. 14:135-141

11. Shectman JM, Nadkarni MM, Voss JD. The association between diabetes metabolic control and drug adherence in an indigent population. Diabetes Care $2002 ; 25: 1015-1021$

12. Johnson JA, Bootman JL. Drug-related morbidity and mortality- a cost-of-illness model. Arch Intern Med 1995; 155:1949-1956

13. Howard T, Stang P, Lydick E. Increased morbidity and mortality associated with discontinuation of oral antidiabetic therapies (abstract). $35^{\text {th }}$ Annual Meeting of the European Association for the study of Diabetes, Brussels, Belgium, 1999

14. Skaer TL, Sclar DA, Markowski DJ, Won JKH. Effect of value added utilities on prescription refill compliance and Medicaid health care expenditures - a study of patients with non-insulin dependent diabetes mellitus. J Clin Pharm Ther $1993 ; 18: 295-299$

15. Brown JB, Nichols GA, Glauber HS, Bakst A. Ten-year follow up of antidiabetic drug use, nonadherence, and mortality in a defined population with type-2 diabetes mellitus. Clin Ther 1999; 21:1045-1057 
16. Bocuzzi SJ, Wogen J, Fox J, Sung JC, Shah AB, Kim J. Utilization of oral hypoglycemic agents in a drug-insured US population. Diabetes Care 2001; 24: $1411-1415$

17. Donnan PT, MacDonald TM, Morris AD. Adherence to prescribed oral hypoglycemic medication in a population of patients with type 2 diabetes: a retrospective cohort study. Diabet Med 2002; 19:279-284

18. Venturini F, Nichol MB, Sung JCY, Bailey KL, Cody M, McCombs JS. Compliance with sulfonylureas in a Health Maintenance Organization: A pharmacy record-based study. Ann Pharmacother 1999; 33:281-288

19. Becker MH, Maiman LA, Kirscht JP, Haefner DP, Drachman RH, Taylor DW. Patient perception and compliance: recent studies of the Health Belief Model. In: Haynes RB, Taylor DW, Sackett DL, eds. Compliance in health care. Baltimore: John Hopkins University Press, 1979:78-112

20. Paes AHP, Bakker A, Soe-Agnie CJ. Impact of dosage frequency in patient compliance. Diabetes Care 1997; 20:1512-1517

21. Nagasawa M, Smith MC, Barnes JH Jr, Fincham JE. Meta-analysis of correlates of diabetes patients' compliance with prescribed medications. Diabetes Educ $1990 ; 16: 192-200$

22. Haynes RB. Determinants of compliance: the disease and the mechanics of treatment. In: Haynes RB, Taylor DW, Sackett DL, eds. Compliance in health care. Baltimore: John Hopkins University Press, 1979:49-62 
23. DiMatteo RM, Lepper HS, Croghan TW. Depression is a risk factor for noncompliance with medical treatment. Meta-analysis of the effects of anxiety and depression on patient adherence. Arch Intern Med 2000; 160:2101-2107

24. Waxman HM, McCreary G, Weinrit RM, Carner EA. A comparison of somatic complaints among depressed and nondepressed older patients. Gerontologist $1987 ; 85: 501-557$

25. Katon W, Von Korff M, Lin E, Walker E, Simon G, Bush T, Robinson P, Russo J. Collaborative management to achieve treatment guidelines. Impact on depression in primary care. JAMA 1995; 273:1026-1031

26. Sherbourne CE, Hays RD, Ordway L, Dimatteo MR, Kravitz RL. Antecedents of adherence to medical recommendations: results from the Medical outcomes Study. J Behav Med 1992; 15:227-268

27. Depression Guideline Panel. Depression in primary care: detection and diagnosis, vol.1. Rockville (MD): US Department of Health and Human Services, Public Health Service, Agency for Health Care Policy and Research, 1993 (AHCPR publication 93-0550)

28. Ciechanowski PS, Katon WJ, Russo JE. Depression and diabetes: Impact of depressive symptoms on adherence, function, and costs. Arch Intern Med 2000; $160: 3278-3285$

29. Anderson RJ, Freedland KE, Clouse RE, Lustman PJ. The prevalence of comorbid depression in adults with diabetes: A meta-analysis. Diabetes Care $2001 ; 24: 1069-1078$ 
30. Talbot F, Noewen A. A review of the relationship between depression and diabetes in adults. Is there a link? Diabetes Care 2000; 23(10): 1556-1562

31. Herbert TB, Cohen S. Depression and immunity: a meta-analytic review. Psychol Bull 1993; 113: 472-486

32. Eaton WW, Armenian HK, Gallo JJ, Pratt L, Ford D. Depression and risk of onset of type-2 diabetes: a prospective, population-based study. Diabetes Care 1996; 19(10): 1097-1102

33. Kawakami N, Shimizu H, Takatsuka N, Ishibashi H. Depressive symptoms and occurrence of type-2 diabetes among Japanese men. Diabetes Care 1999; 22(7): 1071-1076

34. Saydah SH, Brancati FL, Golden SH, Fradkin J, Harris MI. Depressive symptoms and the risk of type 2 diabetes mellitus in a US sample. Diabetes/Met Res Rev 2003; 19:202-208

35. Carnethon MR, Kinder LS, Fair JM, Stafford RS, Fortmann SP. Symptoms of depression as a risk factor for incident diabetes: Findings from the National health and nutrition examination epidemiologic follow-up study, 1971-1992. Am J Epidemiol 2003; 158(5): 416-423

36. Motheral BR, Fairman KA. The use of claims databases for outcomes research: Rationale, challenges, and strategies. Clin Ther 1997; 19(2): 346-366

37. McKenzie DA, Semradek J, McFarland BH, Mullooly JP, McCamant LE. The validity of Medicaid pharmacy claims for estimating drug use among elderly nursing home residents: The Oregon experience. J Clin Epidemiol 2000; $53: 1248-1257$ 
38. Hamilton RA, Briceland LL. Use of prescription-refill records to assess patient compliance. Am J Hosp Pharm 1992; 49:1691-1696

39. Wing RR, Phelan S, Tate D. The role of adherence in mediating the relationship between depression and health outcomes. J Psychosom Res 2002; 53:877-881

40. Lustman PS, Clouse RE, Carney RM. Depression and the reporting of diabetes symptoms. Int J Psychiatry Med 1988; 18:295-303

41. Katon W, Ciechanowski P. Impact of major depression on chronic medical illness. J Psychosom Research 2002; 53: 859-863

42. Chiba M, Suzuki S, Hinokio Y, Hirai M, Satoh Y, Tashiro A, Utsumi A, Awata T, Hongo M, Toyota T. Tyrosine hydroxylase gene microsatellite polymorphism associated with insulin resistance in depressive disorder. Metabolism 2000; 49:1145-1149

43. Okamura F, Tashiro A, Utumi A, Imai T, Suchi T, Tamura D, Sato Y, Suzuki S, Hongo M. Insulin resistance in patients with depression and its changes during the clinical course of depression: minimal model analysis. Metabolism 2000; 49:1255-1260

44. Blumenthal JA, Williams RS, Wallace A, Williams RB Jr, Needles TL. Physiological and psychological variables that predict compliance to prescribed exercise therapy in patients recovering myocardial infarction. Psychosom Med $1982 ; 44: 519-527$

45. Anda RF, Williams on DF, Escobedo LG, Mast EE, Giovino GA, Remington PL. Depression and the dynamics of smoking. JAMA 1990; 264:1541-1545 
46. Davidson JR, Meltzer-Brody SE. The underrecognition and undertreatment of depression: what is the breadth and depth of the problem? J Clin Psychiatry 1999; 60 (Suppl 7): 4-9

47. Hirschfeld RM, Keller MB, Panico S, Arons BS, Barlow D, Davidoff F, Endicott J, Froom J, Goldstein M, Gorman JM, Marek RG, Maurer TA, Meyer R, Phillips K, Ross J, Schwenk TL, Sharfstein SS, Thase ME, Wyatt RJ. The National Depressive and Manic-Depressive Association consensus statement on the undertreatment of depression. JAMA 1997; 277(4): 333-40

48. Peyrot M, McMurry J, Kruger D. A biopsychosocial model of glycemic control in diabetes: Stress, coping, and regimen adherence. J Health Soc Behav 1999; 40: $141-158$

49. Nurymberg K, Kreitler S, Weissler K. The cognitive orientation of compliance in short and long-term type 2 diabetic patients. Patient Educ Counsel 1996; 29:25-39

50. Glasgow RE, Hampson SE, Strycker LA, Ruggiero L. Personal-model beliefs and social-environmental barriers related to diabetes self-management. Diabetes Care 1997; 10: 556-561

51. Toljamo M, Hentinen M. Adherence to self-care and social support. J Clin Nurs 2001; 10(5): 618-627.

52. Breslau N. Depressive symptoms, major depression, and generalized anxiety: a comparison of self-reports on CES-D and results from diagnostic interviews. Psychiatry Res 1985; 15(3): 219-229 
53. McQuaid JR, Stein MB, McCahill M, Laffaye C, Ramel W. Use of brief psychiatric screening measures in a primary care sample. Depression Anxiety $2000 ; 12(1): 21-29$

54. Report of the expert committee on the diagnosis and classification of diabetes mellitus. Diabetes care 1997; 20:1183-1197

55. Campbell IW. Epidemiology and clinical presentation of type 2 diabetes. Value in Health 2000; 3 (Supp 1): S3-S6

56. Yki-Jarvinen H. Pathogenesis of non-insulin dependent diabetes. Lancet 1994; $343: 91-95$

57. Reaven GM. Role of insulin resistance in human disease. Diabetes 1988; $37: 1595-1607$

58. Taylor SI, Accili D, Imai Y. Insulin resistance or insulin deficiency. Which is the primary cause of NIDDM. Diabetes $1994 ; 43: 735-740$

59. Duncan C, Chalmers J, Campbell IW, Jones IG. An audit of non-insulin dependent diabetes attending a district general hospital diabetic clinic: implications for shared care between hospital and general practice. Health Bull 1992; 50(4): 302-308

60. Klein R, Klein BE, Moss SE. Relation of glycemic control to diabetic microvascular complications in diabetes mellitus. Ann Intern Med 1996; 124(1 Pt 2): 90-96

61. Klein R. Hyperglycemia and microvascular and macrovascular disease in diabetes. Diabetes Care 1995; 18(2): 258-268 
62. Orchard TJ. From diagnosis and classification to complications and therapy. DCCT. Part II? Diabetes Control and Complications Trial. Diabetes Care 1994; $17: 326-328$

63. American Diabetes Association: Tests of glycemia in diabetes. Diabetes Care 2001; 24 (Suppl 1): 80-82

64. Turner RC, Cull CA, Frighi V, Homan RR. Glycemic control with diet, sulfonylurea, metformin or insulin in patients with type 2 diabetes mellitus. Progressive requirement for multiple therapies (UKPDS 49). JAMA 1999; 281:2005-2012

65. Graham-Clarke P, Mullen K. New antidiabetic medications: Clinical evidence, clinical practice and pharmacoeconomics. Aust Fam Phys 2001; 30(12): 11451148

66. Boule NG, Haddad E, Kenny GP, Wells GA, Sigal RJ. Effects of exercise on glycemic control and body mass in type 2 diabetes mellitus: a meta-analysis of controlled clinical trials. JAMA 2001; 286(10): 1218-1227

67. American Diabetes Association. Translation of the diabetes nutrition recommendations for health care institutions. Diabetes Care 1997; 20:106-108

68. Tinker LF, Heins JM, Holler HJ. Commentary and translation: 1994 nutrition recommendations for diabetes: Diabetes care and education, a practice group of the American Dietetic Association. J Am Diet Assoc 1994; 94: 507-511

69. Gutowski C. Understanding new pharmacologic therapy for type 2 diabetes. Nurse Practitioner 1999; 24(6): 15-36 
70. Quinn L. Pharmacologic management of the patient with type 2 diabetes. Nurs Clin North America 2001; 36(2): 217-242

71. Zimmerman BR, Hagen MD. An evaluation of new agents in the treatment of type 2 diabetes. J Fam Practice 1998; 47(5): S37-S43

72. Inzucchi SE. Oral antihyperglycemic therapy for type 2 diabetes. JAMA 2002; 287(3): 360-372

73. Evans A, Krentz AJ. Benefits and risks of transfer from oral agents to insulin in type 2 diabetes mellitus. Drug Safety 1999; 21(1): 7-22

74. ACE Consensus Conference on Guidelines for glycemic control. Endocr Pract 2002; 8 (Suppl 1): 5-11

75. DeFronzo R. Pharmacologic therapy for type 2 diabetes. Ann Intern Med 1999; 131: 281

76. Pigon J, Giacca A, Ostenson CG, Lam L, Vranic M, Efendic S. Normal hepatic insulin sensitivity in lean, mild, non-insulin-dependent diabetic patients. J Clin Endocrinol Metab 1996; 81:3702-3708

77. Ohkubo Y, Kishikawa H, Araki E, Miyata T, Isami S, Motoyoshi S, Kojima Y, Furuyoshi N, Shichiri M. Intensive insulin therapy prevents the progression of diabetic microvascular complications in Japanese patients with non-insulin dependent diabetes mellitus: a randomized prospective 6-year study. Diabetes Res Clin Pract 1995; 28:103-117

78. Gray A, Raikou M, McGuire A, Fenn P, Stevens R, Cull C, Stratton I, Adler A, Holman R, Turner R. Cost effectiveness of an intensive blood glucose control 
policy in patients with type 2 diabetes. Economic analysis alongside randomized controlled trial (UKPDS 41). BMJ 2000; 320:1373-1378

79. Palmer AJ, Sendi PP, Spinas GA. Applying some UKPDS results to Switzerland: The cost effectiveness of intensive glycemic control with metformin versus conventional control in overweight patients with type 2 diabetes. Schweiz Med Wochenschr 2000; 130: 1034-1040

80. Testa MA, Simonsen DC. Health economic benefits and QOL during improved glycemic control in patients with type 2 diabetes mellitus: A randomized controlled double-blind trial. JAMA 1998; 280:1490-1496

81. Gilmer TP, O'Connor PJ, Manning WG, Rush WA. The cost to health plans of poor glycemic control. Diabetes Care 1997; 20:1847-1853

82. Wagner EH, Sandhu N, Newton KM, McCulloch DK, Ramsey SD, Grothaus LC. Effect of improved glycemic control on health care costs and utilization. JAMA $2001 ; 285: 182-189$

83. Guillausseau PJ. Influence of oral antidiabetic drugs compliance on metabolic control in type 2 diabetes. A survey in general practice. Diabetes Metab 2003; 29(1): 79-81

84. Hepke KL, Martus MT, Share DA. Costs and utilization associated with pharmaceutical adherence in a diabetic population. Am J Manag Care 2004; 10(part 2): 144-151

85. Balkrishnan R, Rajagopalan R, Camacho FT, Huston SA, Murray FT, Anderson RT. Predictors of medication adherence and associated health care costs in an 
older population with type 2 diabetes mellitus: A longitudinal cohort study. Clin Ther 2003; 25(11): 2958-2971

86. Meichenbaum D, Turk DC. Facilitating Treatment Adherence: A Practitioner's Guidebook. New York: Plenum Publishing Corp; 1987

87. Dezii CM, Kawabata H, Tran M. Effects of once-daily and twice-daily dosing on adherence with prescribed glipizide oral therapy for type 2 diabetes. South Med $J$ 2002; 95(1): 68-71

88. Hamilton RA, Briceland LL. Use of prescription-refill records to assess patient compliance. Am J Hosp Pharm 1992; 49: 1691-1696

89. Schectman JM, Bovbjerg VE, Voss JD. Predictors of Medication-Refill Adherence in an Indigent Rural Population. Med Care 2002; 40(12): 1294-300

90. Dailey G, Kim MS, Lian JF. Patient compliance and persistence with antihyperglycemic drug regimens: Evaluation of a Medicaid patient population with type 2 diabetes mellitus. Clin Ther 2001; 23:1311-1320

91. Sherbourne CD, Hays RD, Ordway L, DiMatteo MR, Kravitz RL. Antecedents of adherence to medical recommendations: results from the Medical Outcomes Study. J Behav Med 1992; 15: 447-468

92. Brown JB, Harris SB, Webster-Bogaert S, Wetmore S, Faulds C, Stewart M. The role of patient, physician, and systemic factors in the management of type 2 diabetes mellitus. Fam Pract 2002; 19:344-349

93. Anderson R. Kirk L. Methods of improving patient compliance in chronic disease states. Arch Intern Med 1982; 142: 1673-1675 
94. Polednak AP. Racial and ethnic differences in disease. New York. Oxford University Press, 1989

95. Ayanian JZ, Udvarhelyi IS, Gatsonis CA, Pashos CL, Epstein AM. Racial differences in the use of revascularization procedures after coronary angiography. JAMA 1993; 269: 2642-2646

96. Eggers PW, Greenberg LG. Racial and ethnic differences in hospitalization rates among aged Medicare beneficiaries, 1998. Health Care Fin Rev 2000; 21:91-105

97. Billups SJ, Malone DC, Carter BL. The relationship between drug therapy noncompliance and patient characteristics, health-related quality of life, and health care costs. Pharmacother 2000; 20: 941-949

98. Bailey JE, Lee MD, Somes GW, Graham RL. Risk factors for antihypertensive medication refill failure by patients under Medicaid managed care. Clin Ther 1996; 18: 1252-1262

99. Monane M, Bohn RL, Gurwitz JH, Glynn RJ, Levin R, Avorn J. Compliance with antihypertensive therapy among elderly Medicaid enrollees: The roles of age, gender, and race. Am J Public Health 1996; 86: 1805-1808

100. Gurwitz JH, Glynn RJ, Monane M, Everitt DE, Gilden D, Smith N, Avorn J. Treatment for glaucoma: Adherence by the elderly. Am J Public Health 1993; 83: $711-716$

101. Gornick ME. Disparities in Medicare services: Potential causes, plausible explanations, and recommendations. Health Care Financing Rev 2000; 21: 23-43 
102. Blendon RJ, Scheck AC, Donelan K, Hill CA, Smith M, Beatrice D, Altman D. How white and African-Americans view their health and social problems difference experiences, different expectations. JAMA 1995; 273: 341-346

103. LaVeist TA, Nickerson KJ, Bowie JV. Attitudes about racism, medical mistrust, and satisfaction with care among African American and white cardiac patients. Med Care Res Rev 2000; 57 (Suppl 1): 146-161

104. Doescher MP, Saver BG, Franks P, Fiscella K. Racial and ethnic disparities in perceptions of physician style and trust. Arch Fam Med 2000; 9: 1156-1163

105. Fiscella K, Franks P, Clancy CM. Skepticism toward medical care and health care utilization. Med Care 1998; 36: 180-189

106. Steinman MA, Sands LP, Covinsky KE. Self-restriction of medications due to cost in seniors without prescription coverage. J Gen Intern Med 2001; 16: 793 799

107. Wichowski H, Kubach S. The relationship of self-perception of illness and compliance with health care regimens. J Advanced Nurs 1997; 25: 548-553

108. Dimatteo MR, Hays RD, Gritz ER. Patient adherence to cancer control regimens: scale development and initial validation. Psychol Assess 1993; 5: 102112

109. Dimatteo MR. Enhancing patient adherence to medical recommendations. JAMA 1994; 271: 79-83

110. Dimatteo MR, DiNicola DD. Achieving patient compliance. Elmsford, New York: Pergamon Press Inc; 1982 
111. Ziegelstein RC, Fauerbach JA, Stevens SS, Romanelli J, Richter DP, Bush DE. Patients with depression are less likely to follow recommendations to reduce cardiac risk during recovery from a myocardial infarction. Arch Intern Med 2000; 160: 1818-23

112. Carney RM, Freedland KE, Eisen SA, Rich MW, Jaffe AS. Major depression and medication adherence in elderly patients with coronary artery disease. Health Psychol 1995; 14: 88-90

113. Simon GE, Von Korff M, Barlow W. Health care costs of primary care patients with recognized depression. Arch Gen Psychiatry 1995; 52: 850-856

114. Callahan CM, Hui SL, Nienafer NA, Musick BS, Tierney WM. Longitudinal study of depression and health services use among elderly primary care patients. Am Geriatr Soc 1994; 42: 833-838

115. Unutzer J, Katon W, Simon G, Grembowski D, Walker E, Rutter C. Costs of medical care in elderly primary care population. Presented at the $9^{\text {th }}$ annual meeting of the American Association of Geriatric Psychiatry, Tucson, AZ, February, 1996

116. Katon W, Von Korff M, Lin E, Lipscomb P, Russo J, Wagner E. Distressed high utilizers of medical care: DSM-III diagnoses and treatment needs. Gen Hosp Psychiatry 1990; 12:355-362

117. Lustman PJ, Anderson RJ, Kenneth FE, DeGroot M, Carney RM, Clouse RE. Depression and poor glycemic control: A meta-analytic review of the literature. Diabetes Care 2000; 23(7): 934-956 
118. Van der Does FE, De Neeling JN, Snoek FJ, Kostense PJ, Grootenhuis PA, Bouter LM, Heine RJ. Symptoms and well-being in relation to glycemic control in type 2 diabetes. Diabetes Care 1996; 19:204-210

119. Lustman PJ, Griffith LS, Clouse RE, Cryer PE. Psychiatric illness in diabetes mellitus: relationship to symptoms and glucose control. J Nerv Ment Dis 1986; 174: $736-742$

120. Wilson W, Ary DV, Biglan A, Glasgow RE, Toobert DJ, Campbell DR. Psychosocial predictors of self-care behaviors (compliance) and glycemic control in non-insulin dependent diabetes mellitus. Diabetes Care 1986; 9:614-621

121. Peyrot M, Rubin RR. Levels of risks of depression and anxiety symptomatology among diabetic adults. Diabetes Care 1997; 20: 585-590

122. Lustman PJ, Griffith LS, Clouse RE, Freedland KE, Eisen SA, Rubin EH, Carney RM, McGill JB. Effects of nortriptyline on depression and glycemic control in diabetes: results of a double-blind, placebo-controlled trial. Psychosom Med 1997; 59: 241-250

123. Lustman PJ, Griffith LS, Freedland KE, Kiessel SS, Clouse RE. Cognitive behavior therapy for depression in type 2 diabetes mellitus. Ann Intern Med 1998; 129: 613-621

124. DeGroot M, Anderson R, Freedland KE, Clouse RE, Lustman PJ. Association of depression and diabetes complications: A meta-analysis. Psychosom Med 2001; 63: 619-630 
125. Egede LE, Zheng D, Simpson K. Comorbid depression is associated with increased health care use and expenditures in individuals with diabetes. Diabetes Care 2002; 25: 464-470

126. Black SA. Increased health burden associated with comorbid depression in older diabetic Mexican Americans. Diabetes Care 1999; 22(1): 56-65

127. Finkelstein EA, Bray JW, Chen H, Larson MJ, Miller K, Tompkins C, Keme A, Manderscheid R. Prevalence and costs of major depression among elderly claimants with diabetes. Diabetes Care 2003; 26: 415-420

128. Babyak M, Blumenthal JA, Herman S, Khatri P, Doraiswamy M, Moore K, Craighead E, Bladewicz T, Krishnan R. Exercise treatment for major depression: maintenance of therapeutic benefit at 10 months. Psychosom Med 2000; 62: 633638

129. Paffenbarger R, Leung R. Physical activity and personal characteristics associated with depression and suicide in American college men. Acta Psychiatr Scand 1994; 377:16-22

130. Blumenthal JAB, Babyak M, Moore K, Craighead WE, Herman S, Khatri P, Waugh R, Napolitano MA, Forman LM, Appelbaum M, Doraiswamy PM, Krishnan KR. Effects of exercise training on older patients with major depression. Arch Intern Med 1999; 159: 2349-2356

131. Gary TL, Crum RM, Cooper-Patrick L, Ford D, Brancati FL. Depressive symptoms and metabolic control in African-Americans with type 2 diabetes. Diabetes Care 2000; 23:23-29 
132. Amato L, Paolisso G, Cacciatore F, Ferrara N, Canonico S, Rengo F, Varricchio M. Non-insulin dependent diabetes mellitus is associated with a greater prevalence of depression in the elderly. Diab Metab 1996; 22: 314-318

133. Viinamaki H, Niskanen L, Uusitupa M. Mental well-being in people with noninsulin dependent diabetes. Acta Psychiatr Scand 1995; 92:392-397

134. Leedom L, Meehan WP, Procci W, Ziedler A. Symptoms of depression in patients with type 2 diabetes mellitus. Psychosomatics 1991; 32: 280-286

135. Palinkas LA, Barret-Connor E, Wingard DL. Type 2 diabetes and depressive symptoms in older adults: a population based study. Diabet Med 1991; 8:532-539

136. Wing RR, Marcus MD, Blair EH, Epstein LH, Burton LR. Depressive symptomatology in obese adults with type 2 diabetes. Diabetes Care 1990; $13: 170-172$

137. Weyerer S, Hewer W, Pfeifer-Kurda M, Dilling H. Psychiatric disorders and diabetes: results from a community study. J Psychosom Res 1989; 33: 633-640

138. Pouwer F, Beekman ATF, Nijpels G, Dekker JM, Snoek FJ, Kostense PJ, Heine RJ, Deeg DJH. Rates and risks for co-morbid depression in patients with type 2 diabetes mellitus: results from a community-based study. Diabetologia 2003; 46(7): 892-898

139. Nichols GA, Brown JB. Unadjusted and adjusted prevalence of diagnosed depression in type 2 diabetes. Diabetes Care 2003; 26: 744-749

140. Willis T. Diabetes: A Medical Odyssey. New York: Tuckahoe, 1971.

141. National Pharmaceutical Council. Pharmaceutical benefits under state medical assistance programs. National Pharmaceutical Council, Reston, VA, 1998 
142. Coughlin TA, Ku L, Holahan J. Medicaid since 1980: costs, coverage, and the shifting alliance between the federal government and the states. Washington D.C. The Urban Institute Press, 1994

143. Medicaid Consumer Information (2003). Available at: http://cms.gov/medicaid/consumer.asp. Accessed 01/24/2004

144. West Virginia State Health Plan-Financing and Cost Considerations (1999). Available at: http://www.hcawv.org/shpMat/shpFinPiper.PDF Accessed $08 / 12 / 2003$

145. Medicaid Information for West Virginia (2003). Available at: http://www.cms.hhs.gov/medicaid/state.asp?state=WV. Accessed 01/15/2004

146. Medicode Inc. International Classification of Diseases, Ninth Revision, Clinical Modification.6th ed. Salt Lake City, Utah: Medicode Inc; 1999.

147. Rural Urban Continuum codes (2003). Available at: (http://www.ers.usda.gov/Data/RuralUrbanContinuumCodes/). Accessed $02 / 04 / 2004$

148. Charlson ME, Pompei P, Ales KL, MacKenzie CR. A new method of classifying prognostic comorbidity in longitudinal studies: development and validation. J Chronic Dis 1987; 40: 372-83

149. D'Hoore W, Bouckaert A, Tilquin C. Practical considerations on the use of the Charlson comorbidity index with administrative databases. J Clin Epidemiol 1996; 49(12): 1429-1433 
150. Steiner JF, Koepsell TD, Fihn SD, Inui TS. A general method of compliance assessment using centralized pharmacy records. Description and validation. Med Care 1988; 26: 814-823

151. Long SJ. Regression models for categorical and limited dependent variables. Thousand Oaks, CA. Sage publications, 1997

152. Kleinbaum DG. Survival Analysis: A Self-Learning Text. New York. SpringerVerlag, 1996

153. Consumer Price Index. Available at: http://www.bls.gov/cpi/home.htm. Accessed 09/06/2003

154. Halverson R, Palmquist R. The interpretation of dummy variables in semilogarithmic equations. Am Econ Rev 1980; 70: 474-475

155. Kennedy P. Estimation with correctly interpreted dummy variables in semilogarithmic equations. Am Econ Rev 1981; 71: 801

156. MacKinnon D. Analysis of mediating variables in prevention and intervention research. NIDA Research Monograph 1994; 139:127-153

157. Hausman JA. Specification tests in econometrics. Econometrica 1978; 46:12511271

158. Eaton WW, Anthony JC, Gallo JJ, Cai G, Tien A, Romanoski A, Lyketsos C, Chen LS. Natural history of DIS/DSM major depression: the Baltimore ECA follow-up. Arch Gen Psychiatr 1997; 54: 993-999

159. Kenny SJ, Aubert RE, Geiss LS. Prevalence and incidence of non-insulin dependent diabetes. In: Harris M, National Diabetes data group, editors. 
Diabetes in America. $2^{\text {nd }}$ ed. Bethesda, MD. National Institute of Diabetes and Digestive and Kidney Diseases, 2000.pp 47-68

160. Jones JK, Garkin L, Lian JF, Staffa JA, Fletcher AP. Discontinuation of and changes in treatment after start of new courses of antihypertensive drugs: a study of a United Kingdom population. BMJ 1995; 311: 293-295

161. Spoelstra JA, Stolk RP, Heerdink ER, Klungel OH, Erkens JA, Leufkens HGM, Grobbee DE. Refill compliance in type 2 diabetes mellitus: a predictor of switching to insulin therapy? Pharmacoepidemiol Drug Safety 2003; 12: 121-127

162. Matthew DR, Cull CA, Stratton IM, Holman RR, Turner RC. UKPDS 26: Sulphonylurea failure in non-insulin dependent diabetic patients over six years. Diabet Med 1998; 15: 297-303

163. Donnan PT, Steinke DT, Newton RW, Morris AD. Changes in treatment after the start of oral hypoglycemic therapy in type 2 diabetes: a population-based study. Diabet Med 2002; 19: 606-610

164. Morningstar BA, Sketris IS, Kephart GC, Sclar DA. Trends in oral antihyperglycemic and insulin use in the Nova Scotia senior population (19931999). Can J Clin Pharmacol 2002; 9(3): 123-129

165. Wang PS, Bohn RL, Knight E, Glynn RJ, Mogun H, Avorn J. Non-compliance with antihypertensive medications: The impact of depressive symptoms and psychosocial factors. J Gen Intern Med 2002; 17: 504-511

166. Choo PW, Rand CS, Inui TS, Lee ML, Cain E, Cordeiro-Breault M, Canning C, Platt R. Validation of patient reports, automated pharmacy records, and pill 
counts with electronic monitoring of adherence to antihypertensive therapy. Med

Care 1999; 37:846-857

167. Piette JD, Richardson C, Valenstein M. Addressing the needs of patients with multiple chronic illnesses: The case of diabetes and depression. Am J Manag Care 2004; 10(part 2): 152-162 


\section{CURRICULUM VITAE}

IFTEKHAR KALSEKAR

103 ELLEN LANE,

Morgantown, WV 26505

Tel: (304)-685-3353

Email: ikalsekar@hsc.wvu.edu

\section{EDUCATION}

08/1998-08/2004

Doctor of Philosophy (Ph.D.), Department of Pharmaceutical Systems \& Policy, West Virginia University

Dissertation: Epidemiological burden of depression and its impact on adherence to oral hypoglycemic agents and related economic outcomes in patients with type 2 diabetes

06/1994 - 06/1998

Bachelor of Pharmacy (B. Pharm), University of Mumbai (formerly Bombay), India

\section{WORK EXPERIENCE}

05/2002-08/2002

\section{Summer Intern}

GlaxoSmithKline, NC

- Conducted retrospective claims data analysis to examine clinical and economic outcomes of single and dual controller therapy in asthma patients using advanced econometric models such as survival analysis, two-part models, and propensity score matching

- Developed a dossier demonstrating the psychometric properties and use of the Stroke Impact Scale-16 (SIS-16) as a primary endpoint in clinical trials to obtain the FDA approval of SIS-16 as a primary outcome in stroke patients

- Assisted in the development of the formulary submission dossier for approval of an oral hypoglycemic agent in the Indiana and Oregon Medicaid programs

$01 / 2000-02 / 2004$

\section{Research Assistant}

- CDC-funded Prevention Research Center, West Virginia UniversityDeveloped evaluation measures, performed data analysis, assisted in writing grants and manuscripts for evaluation of a school based, adolescent smoking cessation program sponsored by the American Lung Association

- Developed a smoking-specific quality of life instrument for adolescents and assessed the cost-effectiveness of the adolescent smoking cessation program in collaboration with the Centers for Disease Control and Prevention (CDC) 


\section{RESEARCH EXPERIENCE}

11/2003-06/2004

Cardiovascular events and expenditures in patients with type 2 diabetes

Primary consultant on a grant funded by Takeda pharmaceuticals to assess the burden of cardiovascular conditions in patients with type 2 diabetes and the differential effect of choice of therapy on expenditures related to type 2 diabetes

08/2001- 04/2002

QT lengthening in patients with schizophrenia

Principal investigator of an unrestricted educational grant provided by Eli Lilly \& Co. to explore the utilization of QT lengthening drugs in patients with schizophrenia in West

Virginia Medicaid

08/2000-08/200

Burden of Barrett's esophagus: prevalence and cost estimates

Research assistant on a study funded by Pharmacia \& Co. to assess the economic burden of Barrett's esophagus and esophageal cancer in the West Virginia Medicaid population. Assisted in the study design, analyses and publication of the study results

$06 / 2000-07 / 2001$

Assessing the need for smoking cessation programs in West Virginia Medicaid Research assistant on a study funded by the West Virginia Bureau of Medical Services to assess the prevalence, attitudes, and preferences of smokers to aid in developing smoking cessation programs. Assisted in study design, survey development, analyses and publication of the study results

\section{TEACHING EXPERIENCE}

08/1998 - 12/1999

Teaching Assistant at the School of Pharmacy, West Virginia University

- Pharmacy as a Profession: Responsibilities included grading, management of course materials, and facilitation of student projects

- Pharmacy Care Lab \& Patient Health Education: Responsibilities included conducting communication skills exercises, evaluating student verbal and nonverbal patient consultation skills, and grading group presentations

- Outcomes Assessment and Quality Improvement. Responsibilities included lecturing and preparing handouts on topics in pharmacoeconomics such as cost utility analyses

$01 / 10 / 2002$

Evidence-based decision making workshop

Conducted a decision-making workshop on economic evaluations in public health for the employees of West Virginia Bureau for Public Health 


\section{RELEVANT COURSES}

Pharmacoeconomics, Econometrics, SAS, Quality of Life Assessment, Epidemiology, Data Management and Analyses, Survey Research, Health Services Marketing, and Health Behavior Theories

\section{COMPUTER SKILLS}

Statistical packages: SPSS, SAS, HLM

Decision analysis software: DATA TreeAge, @ RISK, Precision Tree

\section{PUBLICATIONS}

Kalsekar I, Miller LA. Comment: Pharmacist intervention enhances adherence to orlistat therapy. The Annals of Pharmacotherapy 2004; 38(3): 512

Horn K, Dino G, Kalsekar I, Fernandes A. Appalachian teen smokers: Not On Tobacco 15 months later. American Journal of Public Health 2004; 94(2): 181-184

Horn K, Dino G, Kalsekar I, Massey CJ, Manzo-Tennant K, McGloin T. Exploring the relationship between mental health and smoking cessation: A study of rural teens. Prevention Science 2004; 5(2): 113-26

Dino GA, Kamal KM, Horn KA, Kalsekar I, Fernandes AW. Stage of change and smoking cessation outcomes among adolescents. Addictive Behaviors 2004; 29(5): 93540

Kalsekar I, Makela E, Moeller K. Analysis of West Virginia Medicaid claims data for the prevalence of medical conditions and use of drugs likely to cause QT prolongation in patients with schizophrenia. Current Therapeutic Research 2003; 64(8): 538-550

Horn K, Fernandes A, Dino G, Massey CJ, Kalsekar I. Adolescent nicotine dependence and smoking cessation outcomes. Addictive Behaviors 2003; 28(4): 769-776

Amonkar M, Kalsekar I, Boyer JG. The economic burden of Barrett's esophagus in a Medicaid population. The Annals of Pharmacotherapy 2002; 36(4): 605-611

Massey CJ, Horn K, Goldcamp J, McCracken A, Dino G, Kalsekar I. School-based teen smoking cessation programs: Recruitment issues in research. Journal of School Health 2003; 73(2): 58-63

Dino G, Horn K, Goldcamp J, Fernandes A, Kalsekar I, Massey CJ. A two-year efficacy study of Not-On- Tobacco in Florida: An overview of program successes in changing teen smoking behavior. Preventive Medicine 2001; 33(6): 600-5 
Horn K, Dino G, Kalsekar I, Mody R. The impact of Not On Tobacco on teen smoking cessation: End-of-program evaluation results, 1998-2003. Journal of Adolescent Research. (In press)

Mody R, Kalsekar I, Madhavan S, Hassan M, Amonkar M. Assessing smoking behavior and attitudes towards smoking cessation in a state Medicaid population. Journal of Health Care for the Poor and Underserved. (Under review)

Hassan M, Kalsekar I, Madhavan S, Mody R, Amonkar M. Determinants of smoking among women of childbearing age in West Virginia Medicaid. Journal of Women's Health. (Under review)

\section{SELECTED POSTER PRESENTATIONS}

Kalsekar I, Makela E, Moeller K. Assessment of utilization of drugs and prevalence of conditions leading to QT lengthening in patients with schizophrenia. Presented at the annual meeting of the American Pharmaceutical Association (APhA), New Orleans, LA, March 2003

Horn KA, Dino GA, Goldcamp J, Kalsekar I, Mody R. The Not On Tobacco teen smoking cessation program: A review of end-of-program findings from 1998-2002. Presented at the annual meeting of the Society for Prevention Research, Washington, D.C., June 2003

Kamal KM, Kalsekar I, Fernandes A, Horn K, Dino G. "Stages of Change and Smoking Cessation Outcomes in Adolescents." Presented at the annual meeting of the National Conference on Tobacco and Health, San Francisco, CA, November 2002

Fernandes A, Kalsekar I, Horn K, Massey CJ, Dino G. Adolescent nicotine dependence and smoking cessation outcomes. Presented at the annual meeting of the Society for Prevention Research, Washington, D.C., May 2001

Joshi AV, Nau D, Kalsekar I. An appraisal of health-related quality of life instruments for use in patients diagnosed with Human Immunodeficiency Virus (HIV) disease. Presented at the annual meeting of the International Society for Pharmacoeconomics and Outcomes Research (ISPOR), Arlington, VA, May 2001

Rosenbluth SA, Kalsekar I, Ma JK. Pharmacy Student Research Conference - Eastern States: A study of presenter's interests, opinions, and careers. Presented at the Annual Meeting of the American Association of Colleges of Pharmacy (AACP), Toronto, Canada, July 2001

Dino G, Horn K, Goldcamp G, Fernandes A, Kalsekar I, Massey CJ. A two-year efficacy study of NOT-ON- TOBACCO in Florida: An overview of program successes in changing teen smoking behavior. Presented at the annual meeting of the Society for Prevention Research, Washington, D.C., May 2001 


\section{REVIEWER}

American Journal of Managed Care

Social Science and Medicine

\section{HONORS/AFFILIATIONS}

Recipient of Graduate Teaching Award, School of Pharmacy, West Virginia University Recipient of Graduate Research Award, School of Pharmacy, West Virginia University Member of Rho Chi Honor Society

Member of International Society for Pharmacoeconomics \& Outcomes Research (ISPOR)

Member of American Pharmaceutical Association (APhA) 